AUS DER ABTEILUNG ANATOMIE UND EMBRYOLOGIE

(PROF. DR. MED. C. VIEBAHN)

IM ZENTRUM ANATOMIE

DER MEDIZINISCHEN FAKULTÄT DER UNIVERSITÄT GÖTTINGEN

\title{
Paläopathologische Untersuchungen an den Kinderskeleten der Mad'arovce-Kultur des frühbronzezeitlichen Gräberfeldes von Jelšovce, Slowakei
}

\author{
INAUGURAL-DISSERTATION \\ zur Erlangung des Doktorgrades \\ der Medizinischen Fakultät \\ der Georg-August-Universität zu Göttingen \\ vorgelegt von \\ ULRIKE WEIHMANN, geb. SEILER \\ aus Hamburg
}

Göttingen 2010 
Dekan:

I. Berichterstatter:

II. Berichterstatter/ -in:

III. Berichterstatter/ -in:

Tag der mündlichen Prüfung:
Prof. Dr. med. C. Frömmel

Prof. Dr. phil. nat. Dr. med. M. Schultz

Prof. Dr. Alt

18. Februar 2013 


\section{$\underline{\text { Inhaltsverzeichnis }}$}

Inhaltsverzeichnis $\quad 1$

Tabellenverzeichnis $\quad 5$

$\begin{array}{ll}\text { Abbildungsverzeichnis } & 8\end{array}$

$\begin{array}{ll}\text { Tafelverzeichnis } & 11\end{array}$

$\begin{array}{ll}\text { Abkürzungsverzeichnis } & 14\end{array}$

$\begin{array}{ll}\text { I. Einleitung } & 15\end{array}$

$\begin{array}{ll}\text { I.1. Fragestellung der Arbeit } & 19\end{array}$

II. Material und Methoden $\quad 20$

$\begin{array}{ll}\text { II.1 Material } & 20\end{array}$

II.1.1 Das frühbronzezeitliche Gräberfeld von Jelšovce 20

$\begin{array}{ll}\text { II.1.1.1 Geographische Lage } & 20\end{array}$

$\begin{array}{ll}\text { II.1.1.2 Entdeckungsgeschichte } & 21\end{array}$

II.1.1.3 Struktur des Gräberfeldes 23

II.1.1.4 Datierung des Gräberfeldes $\quad 24$

$\begin{array}{ll}\text { II.1.2 Die Skeletfunde } & 24\end{array}$

II.2 Methoden $\quad 30$

II.2.1 Paläopathologische Untersuchungsmethoden 30

II.2.1.1 Makroskopische Untersuchung 30

II.2.1.2 Lupenmikroskopische Untersuchung 30

$\begin{array}{ll}\text { II.2.1.3 Endoskopische Untersuchung } & 31\end{array}$

II.2.1.4 Röntgenologische Untersuchung 31

II.2.1.5 Anfertigung von Abgüssen 32

II.2.1.6 Lichtmikroskopische Untersuchung 32

II.2.1.7 Rasterelektronenmikroskopische Untersuchung 32 
II.2.3 Aufbau des Katalogs

III. Ergebnisse

III.1 Altersverteilung der Kinder

$\underline{\text { III.2 Spuren pathologischer Prozesse am Cranium }}$

III.2.1 Schädelanomalien und -dysplasien

III.2.2 Veränderungen der Lamina externa des Schädeldaches

III.2.3 Veränderungen der Lamina interna des Schädeldaches

III.2.4 Erkrankungen der venösen Hirnblutleiter:

III.2.5 Veränderungen an der Schädelbasis

III.2.6 Erkrankungen der Orbita

III.2.7 Veränderungen des Jochbogens

III.2.8 Erkrankungen der Nasenhöhle

III.2.9 Erkrankungen der Nasennebenhöhlen

III.2.10 Erkrankungen des harten Gaumens

III.2.11 Erkrankungen des Mittelohres und des Warzenfortsatzes

$\underline{\text { III.3 Spuren pathologischer Prozesse an Kiefer und Zähnen }}$

III.3.1 Karies

III.3.2 Zahnstein

III.3.3 Parodontopathien

III.3.4.Beziehung zwischen Zahnstein und Parodontopathien

III.3.5 Transversale Schmelzhypoplasien

III.3.6 Transversale Zementhypoplasien 
III.4.1 Veränderungen des Schultergürtels

III.4.1.1 Clavicula

III.4.1.2 Scapula

III.4.2 Veränderungen der oberen Extremitäten

III.4.2.1 Humerus

III.4.2.2 Ulna und Radius

III.4.2.3 Hand

III.4.3 Veränderungen der Rippen

III.4.4 Veränderungen des Brustbeins

III.4.5 Veränderungen der Wirbelsäule

III.4.6 Veränderungen des Beckens

III.4.6.1 Os ilii

III.4.6.2 Os ischii

III.4.7 Veränderungen der unteren Extremitäten

III.4.7.2 Tibia und Fibula

III.4.7.3 Fuß 68

$\underline{\text { III.5 Mangelerkrankungen }}$

III.5.1 infantiler Skorbut = Möller-Barlowsche-Erkrankung

III.5.2 Anämie

III.5.2.1 Merkmale einer Anämie

III.5.3 Rachitis 
IV.1. Vergleichspopulationen $\quad 86$

IV.1.1 Gemeinsamkeiten und Unterschiede der verglichenen Populationen 88

$\begin{array}{ll}\text { IV.2. Altersverteilung } & 93\end{array}$

$\begin{array}{ll}\text { IV.2.1 Kleinkinderdefizit } & 93\end{array}$

IV.2.2. Kindersterblichkeit 95

IV.2.3. Vergleich der Kinder- und Säuglingssterblichkeit in der Bronzezeit $\begin{array}{ll}\text { mit rezenten Daten } & 101\end{array}$

$\begin{array}{ll}\text { IV.3. Zahnerkrankungen } & 103\end{array}$

$\begin{array}{ll}\text { IV.4. Entzündungserkrankungen } & 107\end{array}$

$\begin{array}{ll}\text { IV.5 Mangelerkrankungen } & 118\end{array}$

IV.6 Zusammenfassende Darstellung der Vergleichsergebnisse $\quad 127$

IV.7 Ursachendiskussion der beobachteten Ergebnisse $\quad 128$

$\begin{array}{ll}\text { IV.8 Fallbeispiele } & 136\end{array}$

IV.8.1 Fallbeispiel 1: Skorbut und entzündliche Erkrankungen der pneumatischen $\begin{array}{ll}\text { Räume des Schädels } & 136\end{array}$

$\begin{array}{ll}\text { IV.8.2 Fallbeispiel 2: Skorbut und Anämie } & 137\end{array}$

$\begin{array}{ll}\text { IV.8.3 Fallbeispiel 3: Tumorerkrankung } & 138\end{array}$

IV.8.4 Fallbeispiel 4: perimortales Schädeltrauma 140

IV.8.5 Fallbeispiel 5: Skorbut, meningeale Reizung und Kauterisation 141

$\begin{array}{ll}\text { V. Zusammenfassung } & 150\end{array}$

$\begin{array}{ll}\text { VI. Anhang } & 152\end{array}$

$\begin{array}{ll}\text { Tafeln } & 154\end{array}$

VII. Literaturverzeichnis $\quad 254$ 


\section{Katalog}

Katalog Jelšovce, Kindergräber der Mad’arovce-Kultur

\section{Tabellenverzeichnis}

Tab. 1: Chronologische Auflistung der Entdeckungsgeschichte des Gräberfeldes von Jelšovce

Tab. 2: Liste aller Kinder der Mad'arovce-Kultur von Jelšovce nach Grabnummern sortiert

Tab. 4: Altersverteilung innerhalb der Kinderpopulation der Mad'arovce-Kultur

Tab. 7: Merkmale eines Skorbuts am mazerierten Knochen

7a: Veränderungen am Cranium

7b: Veränderungen am Postcranium

Tab. 8: Merkmale eines Skorbuts nach ihrer Wertigkeit

8a: am Cranium

8b: am Postcranium

Tab. 9: Verteilung der Merkmale zur Diagnostik eines Skorbuts 
Tab. 12: Gemeinsamkeiten und Unterschiede der Populationen der Nitra-, Aunjetitzund Mad'arovce-Kultur von Jelšovce

Tab. 13: Vergleiche der Lebensbedingungen zwischen den untersuchten Populationen aus Niederösterreich und Jelšovce, Wandersleben und İkiztepe

Tab. 14: Altersverteilung der Kinderpopulation von Jelšovce und İkiztepe (in Prozent)

Tab. 15: Vergleich der differenzierten Mortalität der Populationen von Jelšovce mit mittelalterlichen Populationen

Tab. 16: Mortalität von der Geburt bis zum sechsten Lebensjahr in verschiedenen bronzezeitlichen Kulturen

Tab. 17: Säuglings- und Kindersterblichkeit 2000 weltweit

Tab. 18: Das Auftreten von Zahnstein, Parodontopathien und Karies in den frühbronzezeitlichen Populationen von Jelšovce.

Tab. 19: Die Häufigkeit des Auftretens von Karies in verschiedenen bronzezeitlichen, neolithischen und mittelalterlichen Kinderpopulationen.

Tab. 20: Die Häufigkeit des Auftretens von Parodontopathien und Skorbut in verschiedenen bronzezeitlichen, neolithischen und mittelalterlichen Kinderpopulationen.

Tab. 21: Entzündliche Erkrankungen der Nasennebenhöhlen, Mittelohren und des Endocranialraums verschiedener frühbronzezeitlichen Populationen.

Tab. 22: Die Häufigkeiten von Stomatitis und Skorbut bei verschiedenen bronzezeitlichen Kinderpopulationen.

Tab. 23: Detaillierte Aufstellung der Erkrankungen der venösen Hirnblutleiter in Jelšovce-Mad'arovce und Franzhausen-I.

Tab. 24: Das Auftreten verschiedener entzündlicher Erkrankungen in verschiedenen bronzezeitlichen, neolithischen und mittelalterlichen Populationen

24a: Sinusitis maxillaris

24b: Otitis media und Pneumatisationshemmungen

24c: Sinusitis frontalis

24d: Otitis media 
24e: Stomatitis

24f: Mastoiditis

24g: Hirndrucksymptomatik (MA + BZ)

24h: Hirndrucksymptomatik (nur BZ)

24i: Meningeale Reizungen (MA + BZ)

24j: Meningeale Reizungen (nur BZ)

24k: Perisinusitis

Tab. 25: Die Häufigkeiten von verschiedenen Mangelerkrankungen in Nitra-, Aunjetitz- und Mad'arovce-Kultur von Jelšovce.

Tab. 26: Das Auftreten von Skorbut in verschiedenen bronzezeitlichen, neolithischen und mittelalterlichen Kinderpopulationen.

Tab. 27: Das Auftreten von Rachitis in verschiedenen bronzezeitlichen und neolithischen Kinderpopulationen.

Tab. 28: Das Auftreten von Anämie in verschiedenen bronzezeitlichen und neolithischen Kinderpopulationen.

Tab. 29: Das Auftreten von transversalen Schmelzhypoplasien in verschiedenen bronzezeitlichen und neolithischen Kinderpopulationen.

Tab. 30: Das Auftreten von transversalen Schmelzhypoplasien bei den Kinderpopulationen von Nitra-, Aunjetitz- und Mad'arovce-Kultur in Jelšovce.

Tab. 31: Das Auftreten von Cribra orbitalia und transversalen Schmelzhypoplasien in verschiedenen bronzezeitlichen und neolithischen Kinderpopulationen. 


\section{Abbildungsverzeichnis}

Abb. 1: Jelšovce, Lage in der Slowakei

Abb. 2: Jelšovce, Lage im Karpatenbecken

Abb. 3: Gräber der Nitra-Kultur

Abb. 4: Gräber der Aunjetitz-Kultur

Abb. 5: Gräber der Mad'arovce-Kultur

Abb. 6: Altersverteilung Kinder gegenüber Juvenilen und Erwachsenen in der Mad'arovce-Kultur in Jelšovce

Abb. 7: Altersverteilung Kinder gegenüber Juvenilen und Erwachsenen in der Mad'arovce-Kultur in Jelšovce, differenziert nach Altersklassen

Abb. 8: Altersverteilung innerhalb der Kinderpopulation der Mad'arovce-Kultur

Abb. 9: Die Häufigkeiten von Nasennebenhöhlenerkrankungen bei den Kindern der Mad'arovce-Kultur in Jelšovce (in Prozent).

Abb. 10: Die Beziehung zwischen Rhinitis und Sinusitis maxillaris bei den Kindern der Mad'arovce-Kultur in Jelšovce (unter Berücksichtigung der Verdachtsfälle).

Abb. 11: Häufigkeiten von Zahnerkrankungen bei den Kindern der Mad'arovce-Kultur in Jelšovce in Prozent

Abb. 12: Verteilung von Zahnstein bei den Kindern der Mad'arovce-Kultur in Jelšovce in den einzelnen Altersklassen

Abb. 13: Verteilung von Parodontopathien bei den Kindern der Mad'arovce-Kultur in Jelšovce in den einzelnen Altersklassen

Abb. 14: Verteilung von transversalen Schmelzhypoplasien bei den Kindern der 
Abb. 15: Die Beziehung zwischen Zahnstein und Parodontose bei den Kindern der Mad'arovce-Kultur in Jelšovce.

Abb. 16: Das Auftreten der verschiedenen Skorbutmerkmale bei den skorbutkranken Kindern der Mad'arovce-Kultur.

Abb. 17: Das Auftreten der verschiedenen Skorbutmerkmale bei den skorbutkranken Kindern der Mad'arovce-Kultur in abfallender Häufigkeit.

Abb. 18: Altersverteilung der Kinder in der Mad'arovce-Kultur von Jelšovce

Abb. 19: Altersverteilung der Kinder in der Aunjetitz-Kultur von Jelšovce

Abb. 20: Altersverteilung der Kinder in der Nitra-Kultur von Jelšovce

Abb. 21: Vergleich der Absterbekurven im Kindesalter von Nitra-, Aunjetitz- und Mad'arovce-Kultur von Jelšovce

Abb. 22: Vergleich der Absterbekurven im Kindesalter verschiedener bronzezeitlicher und mittelalterlicher Kulturen

Abb. 23: Vergleich der Absterbekurven im Alter von der Geburt bis zum 4. Lebensjahr 101 verschiedener bronzezeitlicher und mittelalterlicher Kulturen

Abb. 24: Entzündliche Erkrankungen der Nasennebenhöhlen, Mittelohren und des Endocranialraumes in den drei frühbronzezeitlichen Kinderpopulationen von Jelšovce

Abb. 25: Entzündliche Erkrankungen des Endocranialraumes in den drei frühbronzezeitlichen Kinderpopulationen von Jelšovce

Abb. 26: Vergleich endocranialer Erkrankungen verschiedener bronzezeitlicher und mittelalterlicher Kinderpopulationen

Abb. 27: Das Auftreten von Anämie, Rachitis und Skorbut in den frühbronzezeitlichen Kinderpopulationen von Jelšovce (Häufigkeit in Prozent).

Abb. 28: Häufigkeitsverteilung von Anämie, Rachitis und Skorbut in den drei frühbronzezeitlichen Kinderpopulationen von Jelšovce (Häufigkeit in Prozent).

Abb. 29: Das Auftreten von Rachitis in verschiedenen bronzezeitlichen und neolithischen Kinderpopulationen (Häufigkeit in Prozent). 
Abb. 30: Das Auftreten von Skorbut in verschiedenen bronzezeitlichen und neolithischen Kinderpopulationen (Häufigkeit in Prozent).

Abb. 31: Das Auftreten von Anämien in verschiedenen bronzezeitlichen und neolith126 ischen Kinderpopulationen (Häufigkeit in Prozent).

Abb. 32: Diagramm der Klimaschwankungen zwischen Neolithikum und Völkerwanderungszeit (nach BOUZEK 1982)

Abb. 33: Diagramm zu Fallbeispiel 1: Skorbut und entzündliche Erkrankungen der pneumatischen Räume des Schädels

Abb. 34: Diagramm zu Fallbeispiel 2: Skorbut und Anämie

Abb. 35: Diagramm zu Fallbeispiel 3: Tumorerkrankung

Abb. 36: Diagramm zu Fallbeispiel 4: Schädelhirntrauma

Abb. 37: Diagramm zu Fallbeispiel 5: Skorbut, meningeale Reizung und Kauterisation Mad'arovce-Kultur von Jelšovce 


\section{Tafelverzeichnis}

Tafel 1: Hirndrucksymptomatik (Grab 281), Variation der Lambdanaht (Grab 318) 154

Tafel 2: Anämie (Grab 81) 156

Tafel 3: ausgeheilte Rachitis (Grab 102) 158

Tafel 4: Sutura metopica und Schädeltrauma I (Grab 253) 160

Tafel 5: Schädeltrauma II (Grab 253), Kauterisation (Grab 228 B), 162

Gefäßatypie frontal (19)

Tafel 6: Auflagerungen der Lamina interna I (Grab 162) 164

Tafel 7: Auflagerungen der Lamina interna II (Gräber 162 und 140 C) 166

Tafel 8: Auflagerungen der Lamina interna III (Grab 140 C) 168

Tafel 9: Tuberkulöse Grübchen I (Grab 212) 170

Tafel 10: Tuberkulöse Grübchen II (Grab 212), Auflagerung im 172 Canalis caroticus (Grab 566)

Tafel 11: Veränderungen im Bereich des Ohres I (Gräber 275 und 318) 174

Tafel 12: poröses Orbitadach I (Grab 72) 176

Tafel 13: poröses Orbitadach II (Grab 72) 178

Tafel 14: poröses Orbitadach III (Grab 336 B) 180

Tafel 15: poröses Orbitadach IV (Gräber 88 und 126) 182

Tafel 16: poröses Orbitadach V (Gräber 299 und 300) 184

Tafel 17: Sinusitis maxillaris und Stomatitis (Gräber 65 A und 334) 186 
Tafel 18: Veränderungen im Bereich des Ohres II (Gräber 65 A und 241), Veränderungen der Clavicula (Grab 616 B)

Tafel 19: Zahnerkrankungen I (Gräber 83, 99, 220, 254 B, 336 B, 616 B)

Tafel 20: Zahnerkrankungen II (Gräber 102, 220, 318)

Tafel 21: Veränderungen an den oberen Extremitäten I (Gräber 65A und 158 A)

Tafel 22: Veränderungen an den oberen Extremitäten II (Gräber 99 und 279 A)

196

Tafel 23: Veränderungen an den oberen Extremitäten III (Gräber 65A und 279 A), Veränderungen an den Beckenknochen I (Grab 65 A)

Tafel 24: Veränderungen an Rippen und Brustbein (Gräber 158 A und 279 A)

Tafel 25: Veränderungen der Wirbelsäule I (Grab 350)

Tafel 26: Veränderungen der Wirbelsäule II (Gräber 126 und 228 B)

Tafel 27: Veränderungen an den Beckenknochen II (Grab 299)

Tafel 28: Veränderungen an den unteren Extremitäten I (Grab 95)

Tafel 29: Auflagerungen an den Beinknochen I (Gräber 220, 334, 604 B) 


\section{Abkürzungsverzeichnis}

\begin{tabular}{|c|c|c|c|}
\hline $\begin{array}{l}\text { Abb. } \\
\text { acust. }\end{array}$ & $\begin{array}{l}\text { Abbildung } \\
\text { acusticus }\end{array}$ & $\begin{array}{l}\text { met. } \\
\text { Min. }\end{array}$ & $\begin{array}{l}\text { metaphysäre } \\
\text { Minuten }\end{array}$ \\
\hline $\mathrm{AK}$ & Aunjetitz-Kultur & MK & Mad'arovce-Kultur \\
\hline ant. & anterior & Juv. & Juvenile \\
\hline ap & anterior - posterior & Mm. & musculi \\
\hline Apert. pirif. & Apertura piriformis & Mon. & Monate \\
\hline Aufl. & Auflagerung & MR & meningeale \\
\hline Aunjet. & Aunjetitz & & Reizungen \\
\hline Bar & Barbing & NB & neugeboren \\
\hline bds. & beidseits & n. Chr. & nach Christus \\
\hline Begr. & Begrenzung & Neolith. & Neolithikum \\
\hline $\mathrm{BZ}$ & Bronzezeit & NK & Nitra-Kultur \\
\hline Duct. & Ductus & Osteophyt. & Osteophyten \\
\hline Erw. & Erwachsene & Per & Pergamon \\
\hline ethm. & ethmoidalis & Pit & Pitten \\
\hline ext. & externus & Pneumat. hemm. & Pneumatisations- \\
\hline Fet. mon. & Fetalmonat & & hemmungen \\
\hline For. & Foramen & por. & porös ausgekleidete \\
\hline Fra-I & Franzhausen-I & post. & posterior \\
\hline front. & frontalis & Proc. & Processus \\
\hline G. & Geburt & PS & Perisinusitis \\
\hline ges. & gesamt & $\mathrm{R}$. & Ramus \\
\hline GfA & Gesellschaft für & S. & Sinus \\
\hline & Anthropologie e.V. & s. & siehe \\
\hline Gm-F & Gemeinlebarn-F & sag. & sagittalis \\
\hline ha & Hektar & SAW & Slowakische \\
\hline Hain & Hainburg & & Akademie der \\
\hline Hart & Harting & & Wissenschaften \\
\hline HD & $\begin{array}{l}\text { Hirndruck- } \\
\text { symptomatik }\end{array}$ & $\begin{array}{l}\text { Sin./sin. } \\
\text { Sinusit. }\end{array}$ & $\begin{array}{l}\text { sinus } \\
\text { Sinusitis }\end{array}$ \\
\hline hrsg. v. & herausgegeben von & S.o. & siehe oben \\
\hline Ind. & Individuen & sphen. & sphenoidale/ -is \\
\hline Inf. & Infans & s.u. & siehe unten \\
\hline infraorb. & $\begin{array}{l}\text { Infraorbitale } \\
\text { internus }\end{array}$ & sub. Hämat. & $\begin{array}{c}\text { subperiostale } \\
\text { Hämatome }\end{array}$ \\
\hline J. & Jahre & Sulc. & Sulcus \\
\hline Jel. & Jelšovce & sup. & superior \\
\hline Jh. & Jahrhundert & Tab. & Tabelle \\
\hline Jochbeinaußenfl. & Jochbeinaußenfläche & Transv. & transversale \\
\hline $\mathrm{kV}$ & Kilovolt & TS & transversale \\
\hline L. & Lamina & & Schmelzhypoplasien \\
\hline $\mathrm{Lj}$. & Lebensjahr & V.a. & Verdacht auf \\
\hline M. & Musculus & v. Chr. & vor Christus \\
\hline MA & Mittelalter & vgl. & vergleiche \\
\hline Mad'arov. & Mad'arovce & z.B. & zum Beispiel \\
\hline $\begin{array}{l}\text { Mangelerkr. } \\
\text { max. }\end{array}$ & $\begin{array}{l}\text { Mangelerkrankungen } \\
\text { maxillaris }\end{array}$ & & \\
\hline
\end{tabular}




\section{Einleitung}

Die Untersuchung von menschlichen Skeletresten liefert einen wertvollen Beitrag zur Rekonstruktion der Lebensweise in der Vorzeit (SCHULTZ 1982). Wo Informationen aus schriftlichen Quellen fehlen, sind Knochenfunde oft die einzige Quelle über den Gesundheitszustand einer Population. Der Anthropologe Ämilian Kloiber (KLOIBER 1957) bezeichnet vorgeschichtliche menschliche Skeletfunde aus diesem Grund als "biohistorische Urkunden" (SCHULTZ 1994 a, 1989 a und 1989 c). Sie geben Hinweise auf die ganz persönliche Geschichte eines Individuums sowie eventuelle in der Gesellschaft praktizierte medizinischtherapeutische Eingriffe (z.B. Trepanationen/ Kauterisationen) (SCHULTZ 1989 c).

Es ist bekannt, dass jeder Mensch durch die Umgebung, in der er lebt, beeinflusst wird; Umweltgegebenheiten wie Wohn- und Arbeitsbedingungen, die Möglichkeiten der Ernährung, hygienische Bedingungen, geographische und klimatische Voraussetzungen, prägen die Lebensqualität eines Menschen (SCHULTZ 1982). Alle diese Faktoren sind auch für das Auftreten und die Verbreitung von Erkrankungen mitverantwortlich. Schon Hippokrates maß den äußeren Umständen eine entscheidende Bedeutung bei der Entstehung von Krankheiten bei. Er weist in seiner Abhandlung "Über Luft-, Wasser- und Ortsverhältnisse" darauf hin, wie wichtig es ist, sich mit den geographischen Gegebenheiten vor Ort zu beschäftigen und diese bei der Behandlung von Patienten zu berücksichtigen (HIPPOKRATES in Kollesch \& Nickel 1994) Daraus lässt sich ableiten, dass vom Auftreten bestimmter Erkrankungen (in unterschiedlicher Ausprägung und Häufigkeit) Rückschlüsse auf die zugrundeliegenden Lebensbedingungen gezogenen werden können (SCHULTZ 1982).

Die Paläopathologie ist eine Wissenschaft im Grenzgebiet zwischen Medizin, Anthropologie und Archäologie (MOODIE 1923). Die Berücksichtigung von Erkenntnissen aus anderen Disziplinen (z.B. Archäologie, Anthropologie, Paläozoologie oder Paläobotanik) über klimatische, soziale 
und wirtschaftliche Verhältnisse in der Vorzeit ist bei der Interpretation der paläopathologischen Befunde unerlässlich (SCHULTZ et al. 1998, SCHULTZ 1989 c und 1994 a).

Erst die Summe der Einzelbefunde aus den Untersuchungen der verschiedenen Disziplinen bildet das Mosaik, aus dem das Bild der früheren Lebensbedingungen und -verhältnissen entsteht. Nur die Berücksichtigung aller Aspekte kann ein möglichst authentisches Bild entstehen lassen.

Da Kinder besonders empfindlich auf Veränderungen der Lebensbedingungen reagieren und von diesen schwerer als andere Mitglieder einer Population betroffen sind, kommt ihnen in der Paläopathologie eine besondere Bedeutung zu (MENSFORTH et al. 1978, SCHULTZ \& SCHMIDT-

SCHULTZ 1994, SCHULTZ 2001 a und 1994 b). Kinder befinden sich im Wachstum und sind daher auf hochwertige Nahrung angewiesen. Von Nahrungsknappheit sind sie deshalb besonders schwer betroffen (CARLI-THIELE 1996); zudem tragen Mangelerkrankungen zur Insuffizienz des noch unvollständig ausgereiften Immunsystems bei, was ebenfalls zu einer Benachteiligung der Kinder führt (SCHULTZ 2001 a und 1987, SCHULTZ \& SCHMIDT-SCHULTZ 1994).

Im Rahmen der vorliegenden Arbeit wurden bronzezeitliche Kinderskelete aus Jelšovce im Nitratal in der Slowakei untersucht. Sie gehören der Mad'arovce-Kultur an, einer südwestslowakischen regionalen Kulturtradition der ausgehenden frühen Bronzezeit (Frühe Bronzezeit in der Slowakei etwa 2200 bis 1500 v. Chr.). Das Nitratal war schon in der Steinzeit besiedelt. Verschiedene steinzeitliche Kulturen, z.B. Menschen der Lengyel-Kultur, LudaniceGruppe, Badener-Kultur und Kosihy-Čaka-Gruppe hinterließen Siedlungsspuren in Form von Resten der Wohnstätten, Vorratsgruben und Bestattungen. Während der Frühbronzezeit bewohnten nacheinander Träger von Nitra-, Aunjetitz- und Mad'arovce-Kultur das Nitratal (FURMÁNEK et al.1999, BÁTORA 2000, BÁTORA \& SCHULTZ 2001).

Die Nitra-Kultur ist eine lokal im Nitratal auftretende Kulturgruppe der beginnenden Bronzezeit und steht am Übergang vom Neolithikum zur Bronzezeit. Sie erstreckte sich in der Südwestslowakei vor allem in den Tälern von Waag und Nitra (FURMÁNEK et al. 1999, BÁTORA 2000). 
Die Aunjetitz-Kultur war seit Beginn des 2. Jahrtausends v. Chr. in Böhmen, Mähren, Niederösterreich, Schlesien, Sachsen, Thüringen, Brandenburg und West-Polen verbreitet. Diese früh- bis mittelbronzezeitliche Kulturgruppe war gekennzeichnet durch reichen Metallbesitz und spezifischen Formenbestand. Es wurden Kupfererzlagerstätten ausgebeutet und das Rohkupfer zu Arsen- und Zinnbronzen legiert (BÁTORA 2000).

Zur Entstehung der Aunjetitz-Kultur in der Slowakei gibt es gegenwärtig zwei Theorien: Die erste geht von einer Invasion von Trägern der Aunjetitz-Kultur aus dem benachbarten Mähren aus, die zweite postuliert eine kontinuierliche Entwicklung aus der Nitra-Kultur heraus unter Beteiligung westlicher, aunjetitzer und südöstlicher Einflüsse (FURMÁNEK et al. 1999).

Die Mad'arovce-Kultur hat sich aus der ansässigen Bevölkerung der Aunjetitz-Kultur entwickelt (FURMÁNEK et al. 1999, BÁTORA 2000). Sie ist mit der mährischen Věteřov-Kultur verwandt und wurde durch befestigte Höhensiedlungen, u.a. in Nitriansky Hrádok, Malé Kosihy und Veselé (alle heutige Slowakei) bekannt (FURMÁNEK et al. 1999). In der Frühphase der Mad'arovce-Kultur wurde die Verwendung von Bronze anstatt von Kupfer üblich. Die Keramikindustrie entwickelte eigene Formen; typisch sind die "klassischen Tassen der Mad'arovce-Kultur" (BÁTORA 2000).

In der Mittelbronzezeit entwickelte sich aus der Mad'arovce-Kultur die karpatische Hügelgräberkultur. Aus dieser gingen während der Spätbronzezeit die mitteldanubischen Urnenfelderkulturen hervor. Das Nitratal wurde in dieser Periode von Trägern der Čaka- und der Lausitzer-Kultur besiedelt (FURMÁNEK et al. 1999, BÁTORA 2000).

In Jelšovce haben Träger der drei frühbronzezeitlichen Kulturen nacheinander gelebt und ihre Toten in unmittelbarer Nähe zueinander bestattet (BÁTORA 2000). Das Gräberfeld von Jelšovce bietet somit die einzigartige Möglichkeit zur komplexen anthropologischen und paläopathologischen Erforschung und zum gegenseitigen Vergleich dieser drei denselben Lebensraum nutzenden Populationen. Die Untersuchungsergebnisse können Hinweise auf Veränderungen der Lebensbedingungen über 700 Jahre im Verlauf der gesamten Frühbronzezeit 
in Jelšovce geben und ermöglichen Aussagen zur Bedeutung des Biotops für die Gesundheit der Menschen (SCHULTZ 1987, SCHULTZ \& SCHMIDT-SCHULTZ 1994, BÁTORA \& SCHULTZ 2001).

Bei der Beurteilung der paläopathologischen Untersuchungsergebnisse ist zu bedenken, dass nur Erkrankungen nachgewiesen werden können, die lange genug bestanden haben, um zu Veränderungen an den Knochen zu führen (ORTNER 2003, WoOD et al. 1992). Bei Kindern führen z.B. meningitische Erkrankungen erst nach acht bis zwölf Tagen zu nachweisbaren Veränderungen des Schädelknochens (SCHULTZ 2001 b). Fulminante Verläufe sind somit nicht nachweisbar (ORTNER 2003, WOOD et al. 1992).

Auch Erkrankungen der Weichteile, die sich nicht am Knochen manifestieren, sind nur zum Teil nachzuweisen, da die Weichteile in der Regel nicht erhalten sind. Daraus ergibt sich, dass zwar ein großer Teil, aber nicht alle prähistorischen Erkrankungen erfasst werden können. Postmortale Zerstörung erschwert ebenfalls die Befundung von prähistorischen Knochenfunden. Somit spiegeln die gemachten Beobachtungen lediglich einen Teil der tatsächlich vorhandenen Erkrankungen wider (SCHULTZ et al. 1998, ORTNER 2003). 


\section{$\underline{\text { I.1. Fragestellung der Arbeit }}$}

Ziel dieser Arbeit ist es, die Kinderskelete der Mad'arovce-Kultur des frühbronzezeitlichen Gräberfeldes von Jelšovce auf Spuren krankhafter Veränderungen zu untersuchen, um Kenntnisse über den Gesundheitszustand der Kinder zu erlangen und Rückschlüsse auf deren Lebensbedingungen zu ziehen. Zudem sollen die Kinderskelete der Mad'arovce-Kultur mit denen der Aunjetitz- und Nitra-Kultur aus Jelšovce und anderen bronzezeitlichen, neolithischen sowie mittelalterlichen Populationen verglichen werden.

Dabei soll untersucht werden, ob und in wieweit sich die Lebensbedingungen im Laufe der Zeit verändert haben. Für Unterschiede in Mortalität und Morbidität sollen mögliche Erklärungen aufgezeigt werden.

Der Skorbut wird in dieser Arbeit bevorzugt behandelt, da zum einen ein System zur Diagnose von kindlichem Skorbut vorgestellt wird, welches eine Diagnosefindung erleichtern soll. Zum anderen treten in der Mad'arovce-Kultur in Jelšocve im Vergleich zu den anderen Mangelerkrankungen relativ viele Fälle von Skorbut auf, so dass sich hier eine genauere Betrachtung anbietet. Außerdem kann ein Skorbut als möglicher Cofaktor die hohe Prävalenz an entzündlichen Erkrankungen in der Mad'arovce-Kultur mit erklären. 


\section{$\underline{\text { II. Material und Methoden }}$}

\section{II.1 Material}

\section{II.1.1 Das frühbronzezeitliche Gräberfeld von Jelšovce}

\section{II.1.1.1 Geographische Lage}

Das frühbronzezeitliche Gräberfeld von Jelšovce liegt im Nitratal im nordwestlichen Karpatenbecken im Südwesten der heutigen Slowakei (Abb. 1 und 2, BÁTORA 2000). Der Ort Jelšovce im Bezirk Nitra ist ein kleiner Ort 12 Kilometer nordwestlich der Stadt Nitra. Er liegt an der Hauptstraße von Nitra nach Topol'čany, am westlichen Ufer des Flusses Nitra auf einer 2,5 bis 3 m hohen Löß-Sand-Terrasse. Das Gräberfeld liegt im östlichen Teil des Geländes eines großen ehemaligen LPG-Wirtschaftshofes am Nordrand des heutigen Ortes. Der Süd- und Ostteil des Gräberfeldes befindet sich im ackerbaulich genutzten Bereich. Bei den Grabungen unter der Leitung von Prof. Dr. Jozef Bátora in den Jahren 1982 bis 1987 wurden auf einer Fläche von 3,7 ha 642 Gräber entdeckt und untersucht, von denen 616 in die frühe Bronzezeit datiert wurden. Diese frühbronzezeitlichen Gräber werden drei unterschiedlichen, auf einander folgenden Kulturen zugeordnet, der Nitra-, der Aunjetitz- und der Mad'arovce-Kultur. Es handelt sich um eines der größten bisher bekannten Gräberfelder aus der frühen Bronzezeit im nördlichen Karpatenbecken. Ursprünglich bestand das Gräberfeld aus etwa 1000 Gräbern, durch die Überbauung in den 50er und 60er Jahren des 20. Jh. und durch landwirtschaftliche Nutzung wurde eine nicht unerhebliche Anzahl der Gräber zerstört. Die Gräber waren zum Teil mit reichen Grabbeigaben ausgestattet. Es wurden Schmuck- und Gebrauchsgegenstände aus Kupfer, Bronze, Keramik, Horn und Eberzähnen gefunden. Zahlreiche Gräber wurden schon in früheren Zeiten ausgeraubt. Neben den Gräbern wurden auf derselben Fläche noch 572 ur- und frühgeschichtliche Siedlungsobjekte aus 14 unterschiedlichen Kulturen und Kulturgruppen entdeckt. Es zeigte 
sich, dass dieses Gebiet während beinahe des gesamten Paläolithikums bis ins frühe Mittelalter hinein fast ununterbrochen intensiv besiedelt wurde (BÁTORA 2000).

\section{II.1.1.2 Entdeckungsgeschichte}

Tab. 1 Chronologische Auflistung der Entdeckungsgeschichte des Gräberfeldes von Jelšovce

\begin{tabular}{|c|c|}
\hline $1951 / 52$ & $\begin{array}{l}\text { Eine befestigte Siedlung der Mad’arovce-Kultur wurde von J. Porubský und J. } \\
\text { Kudláček untersucht. Sie lag } 500 \text { m vom Gelände des Wirtschaftshofes entfernt (bei } \\
\text { der heutigen Hydrozentrale). }\end{array}$ \\
\hline 1959 & $\begin{array}{l}\text { J. Rajček und K. Pieta, Mitarbeiter des Archäologischen Instituts der Slowakischen } \\
\text { Akademie der Wissenschaften in Nitra (SAW), entdeckten im Profil einer } \\
\text { Silagegrube eine Siedlungsgrube der Badener Kultur. }\end{array}$ \\
\hline 1962 & $\begin{array}{l}\text { In einer weiteren Silagegrube wurde eine Kulturschicht mit Keramikfunden aus der } \\
\text { Čaka-Kultur entdeckt. }\end{array}$ \\
\hline 1963 & $\begin{array}{l}\text { Bei Baggerarbeiten wurden Siedlungsobjekte und Gräber der Nitra- und } \\
\text { Mad'arovce-Kultur zerstört. }\end{array}$ \\
\hline Herbst 1981 & $\begin{array}{l}\text { Bei Kanalbauarbeiten wurden mehrere Gräber angeschnitten, diverser } \\
\text { Kupferschmuck, ein Knochenpfriem und zwei Eberzähne konnten gerettet werden. }\end{array}$ \\
\hline $\begin{array}{l}\text { November } \\
1981 \text { und } \\
\text { Frühling } \\
1982\end{array}$ & $\begin{array}{l}\text { Begehungen durch J. Bátora und M. Antala bzw. J. Urbanec, bei denen Reste eines } \\
\text { gestörten Grabes der Mad'arovce-Kultur und Siedlungsobjekte aus der Kosihy- } \\
\text { Čaka-Gruppe sowie Siedlungshinweise der Ludanice-Gruppe und der Badener } \\
\text { Kultur gefunden wurden, fanden statt. }\end{array}$ \\
\hline 1982-1987 & $\begin{array}{l}\text { Unter der Leitung von J. Bátora vom Archäologischen Institut der SAW zu Nitra } \\
\text { fanden Ausgrabungen auf dem Gelände des Wirtschaftshofes statt. }\end{array}$ \\
\hline
\end{tabular}


Abb. 1 Jelšovce, Lage in der Slowakei (BÁTORA 2000, S. 13)

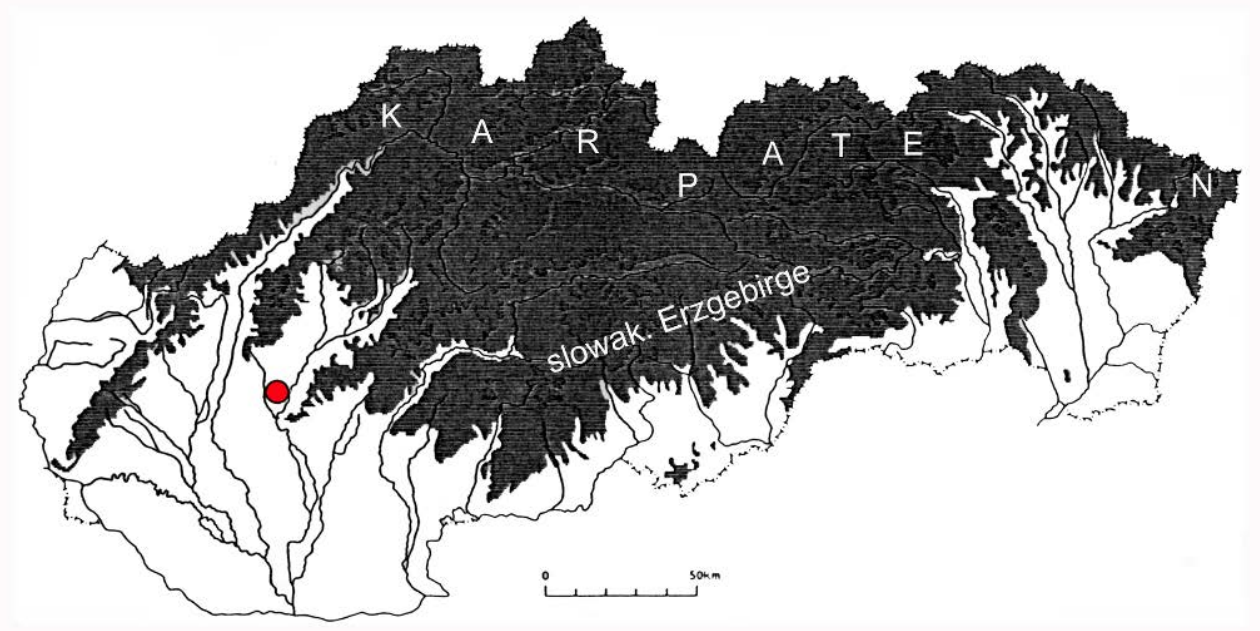

roter Punkt: Jelšovce, schwarze Flächen: Gebirge, schwarze Striche: Flüsse

Abb. 2 Jelšovce, Lage im Karpatenbecken (BÁTORA 2000, S. 13)

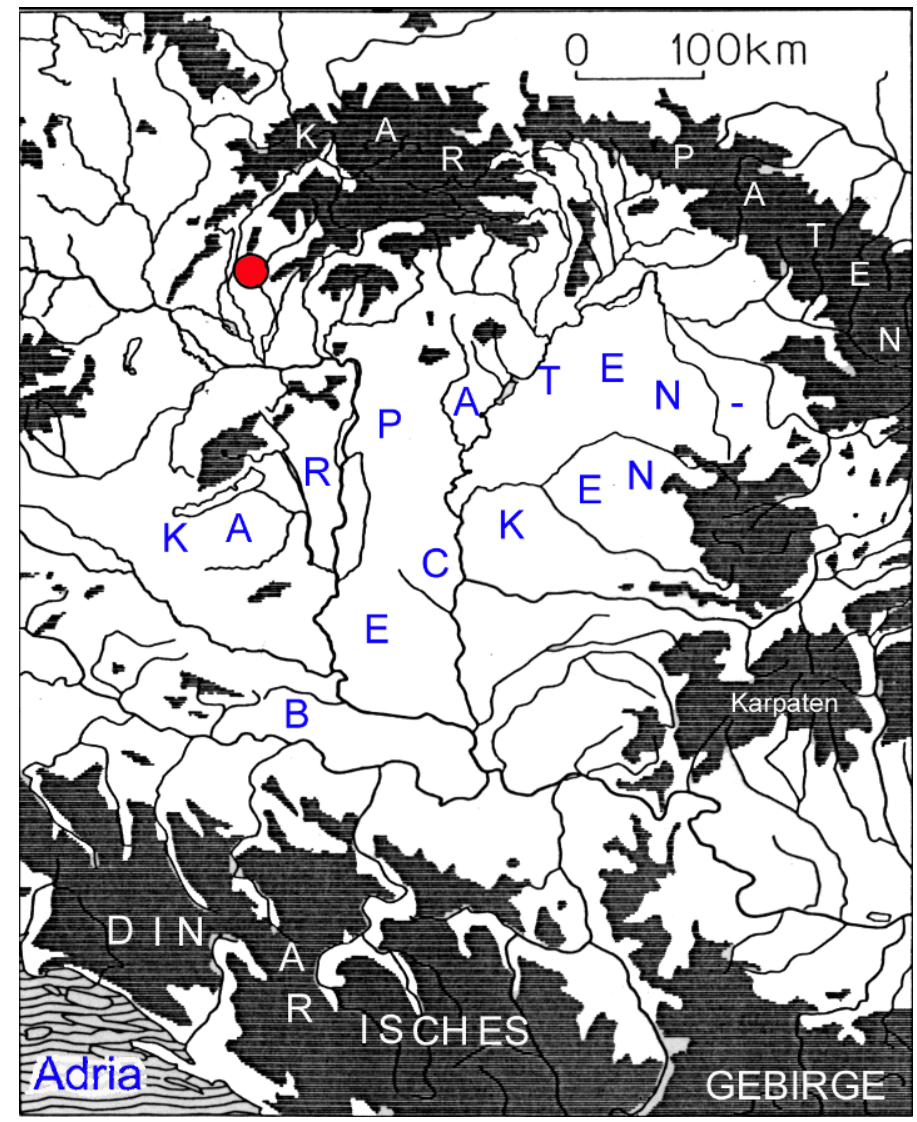

roter Punkt: Jelšovce, schwarze Flächen: Gebirge, schwarze Striche: Flüsse 


\section{$\underline{\text { II.1.1.3 Struktur des Gräberfeldes }}$}

Insgesamt waren die Gräber über eine sehr große Fläche verteilt. Die nördlichste Bestattung lag etwa 300 m von der südlichsten entfernt. Der Abstand zwischen dem östlichsten und dem westlichsten Grab betrug etwa 270 m. Die Belegungsbereiche der drei frühbronzezeitlichen Kulturen lassen sich relativ gut voneinander abgrenzen. Es können drei separate Gräberfeldbereiche sowie vier kleinere Gräbergruppen unterschieden werden. Leider wurden alle Bereiche teilweise durch den Bau landwirtschaftlicher Gebäude gestört oder durch Betonflächen und Straßenbelag überdeckt. Die Tatsache, dass es kaum zu Grabstörungen durch nachfolgende Kulturen gekommen ist, lässt die Schlussfolgerung $\mathrm{zu}$, dass die Gräber der jeweils vorausgegangenen Kulturen für die nachfolgenden sichtbar waren und von diesen respektiert wurden (BÁTORA 2000).

Die Gräber der Nitra-Kultur sind durch zwei selbständige, etwa 170 m voneinander entfernte Gräberfeldareale und zwei kleinere Gräbergruppen repräsentiert. Eine der Gräbergruppen befindet sich zentral im großen Gräberfeldareal der Mad'arovce-Kultur. Die übrigen Gräber befinden sich im nördlichen und nordwestlichen Bereich des Gräberfeldes (Abb. 3, BÁTORA 2000).

Die Aunjetitz-Kultur ist in einer selbständigen, auf der Nordseite durch eine 14 bis $18 \mathrm{~m}$ breite Lücke von den nitrazeitlichen Gräbern getrennte Gräberfeldgruppe vertreten. Auf der Südseite schließt sich das mad'arovcezeitliche Gräberfeld lückenlos an (Abb. 4, BÁTORA 2000).

Das Gräberfeld der Mad'arovce-Kultur erstreckt sich auf eine große Fläche des südlichen und östlichen Teils des untersuchten Areals. Eine kleine Gruppe von 13 Gräbern befindet sich im nördlichen Gräberfeldareal der Nitra-Kultur (Abb. 5, BÁTORA 2000). Der Südrand des Gräberfeldes war besonders durch die landwirtschaftliche Nutzung des Bodens betroffen und wurde teilweise zerstört. Im Gegensatz zu meist in Reihen angeordneten Gräbern der Nitra- und Aunjetitz-Kultur finden sich bei der Mad'arovce-Kultur vorwiegend in Gruppen angeordnete Gräber. Dies lässt Rückschlüsse auf Veränderungen in der Sozialstruktur und in den 
Familienbeziehungen der Gemeinschaft zu. Im zentralen Gräberfeldbereich der Mad'arovceKultur wurden vier ursprünglich überhügelte Gräber gefunden, die diese Annahme zusätzlich bestätigen (BÁTORA 2000).

\section{II.1.1.4 Datierung des Gräberfeldes}

Die Belegung des frühbronzezeitlichen Gräberfeldes erfolgte von etwa 2200 v. Chr. bis 1500 v. Chr. Die nitrazeitliche Belegung beginnt etwa 2200 bis 2150 v. Chr. Der Übergangsbereich zwischen Nitra- und Aunjetitz-Kultur wird auf etwa 1930 bis 1870 v. Chr. datiert. Die klassische Phase der Aunjetitz-Kultur dauerte etwa bis 1730 v. Chr. Die Zeit, in der das Gräberfeld nicht genutzt wurde, betrug zwischen den einzelnen Kulturen nur ca. 50 bis 100 Jahre.

Die Belegungszeit der Mad'arovce-Kultur endet ungefähr 1500 v. Chr. (BÁTORA 2000, GÖRSDORF 2000). Die etwa 700 jährige Belegung des Gräberfeldes repräsentiert die gesamte kulturhistorische Entwicklung der Frühbronzezeit in der Südwestslowakei.

\section{II.1.2 Die Skeletfunde}

Im Rahmen dieser Arbeit wurden menschliche Kinderskelete der letzten und jüngsten frühbronzezeitlichen Kultur, der Mad'arovce-Kultur, untersucht und bearbeitet. Unter den insgesamt 311 mad’arovcezeitlichen Gräbern mit 339 Individuen befanden sich 64 Gräber mit 79 subadulten Individuen. Zwei Gräber mit Resten von vier Kindern (Gräber 26 und 561) konnten leider nicht untersucht werden, da sie zum Zeitpunkt der Untersuchung als verschollen galten. Für die Untersuchung standen somit 75 Individuen der Altersklassen Neonatus bis Infans II zur Verfügung. Davon waren von acht Kindern nur einzelne Knochen als Zusatzfunde in anderen Gräbern erhalten. Diese werden nicht als einzelne Individuen besprochen, sondern lediglich in der Sterbestatistik berücksichtigt. Die Skeletfunde wurden nach der Bergung in der Akademie der Wissenschaften in Nitra von Dr. Julius Jakab anthropologisch bearbeitet, altersbestimmt und für die Ausgrabungsveröffentlichung katalogisiert. Von Prof. Schultz wurden einzelne Gräber 
schon ganz oder teilweise vorab makroskopisch untersucht. Im Einzelnen handelt es sich um folgende Gräber:

vollständig bearbeitet: Grabnummern 88 A, 118, 120, 126, 318, 331, 332, 334, 336 B, 340

teilweise bearbeitet: Grabnummern 81 (Teile des Craniums, Humerus links), 253 (Cranium), 279 B (Postcranium), 299 (Cranium), 338 (Postcranium), 616 B (Postcranium)

Auch diese Skelete wurden von der Autorin nachuntersucht. Zusätzlich wurden ergänzende Untersuchungen durchgeführt (Fotografie, Endoskopie, Röntgen, mikroskopische Untersuchung). 
Abb. 3 Gräber der Nitra-Kultur (gelb markierte Bereiche) (BÁTORA 2000, S. 448)

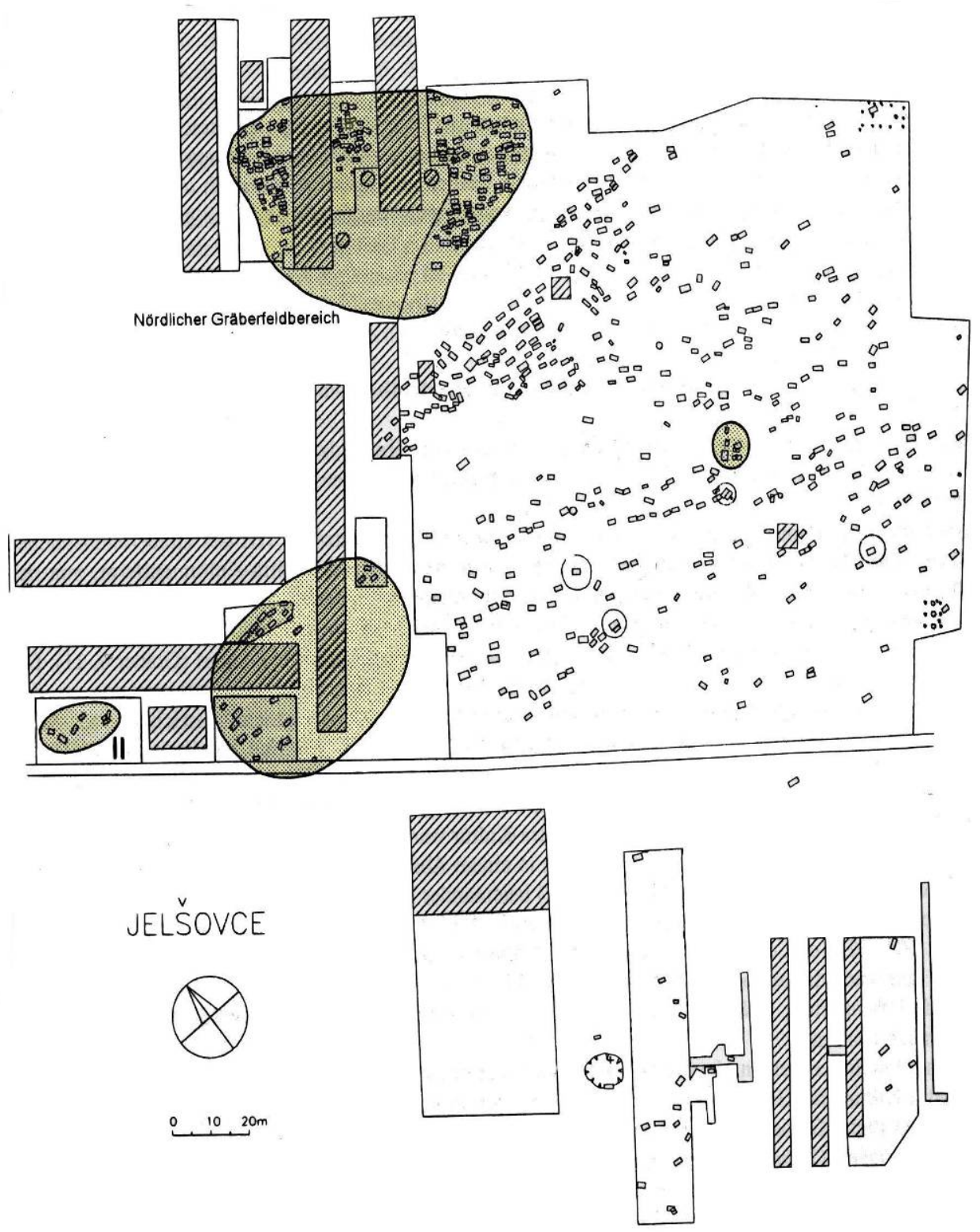

Die Abbildung zeigt das Areal des ehemaligen LPG-Wirtschaftshofes sowie die Grabungsflächen und Gräber in diesem Bereich. Gelb markiert sind die Areale, in denen sich nitrazeitliche Gräber befinden.

schraffierte Bereiche: umrandete Bereiche: kleine Rechtecke:
Gebäude

Ausgrabungsflächen

Grabgruben 
Abb.4 Gräber der Aunjetitz-Kultur (blau markierte Bereiche) (BÁTORA 2000, S. 461)
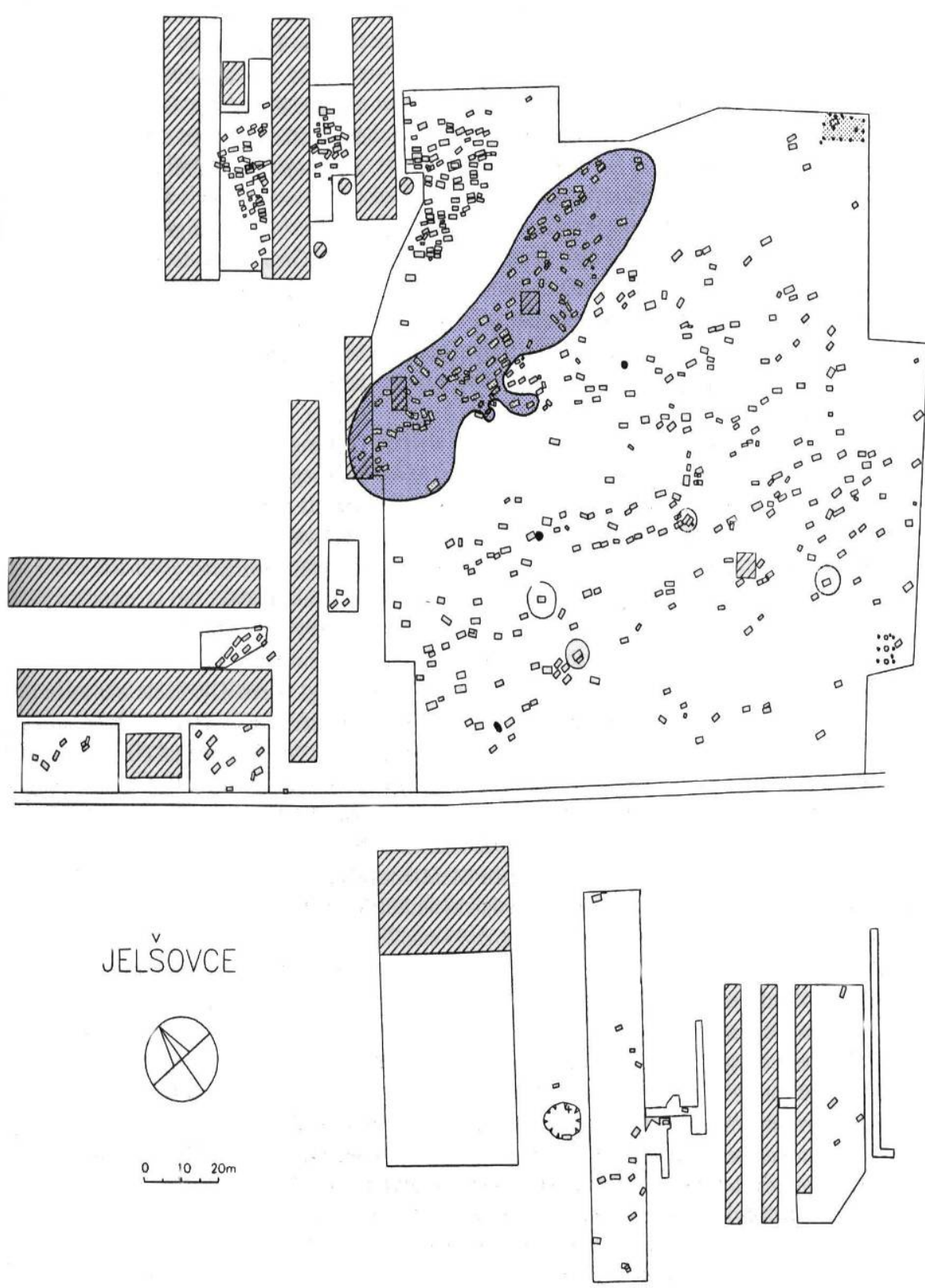

Die Abbildung zeigt das Areal des ehemaligen LPG-Wirtschaftshofes sowie die Grabungsflächen und Gräber in diesem Bereich. Blau markiert sind die Areale, in denen sich aunjetitzzeitliche Gräber befinden.

schraffierte Bereiche:

umrandete Bereiche:

kleine Rechtecke:
Gebäude

Ausgrabungsflächen

Grabgruben 
Abb.5 Gräber der Mad'arovce-Kultur (rot markierte Bereiche) (BÁTORA 2000, S. 472)

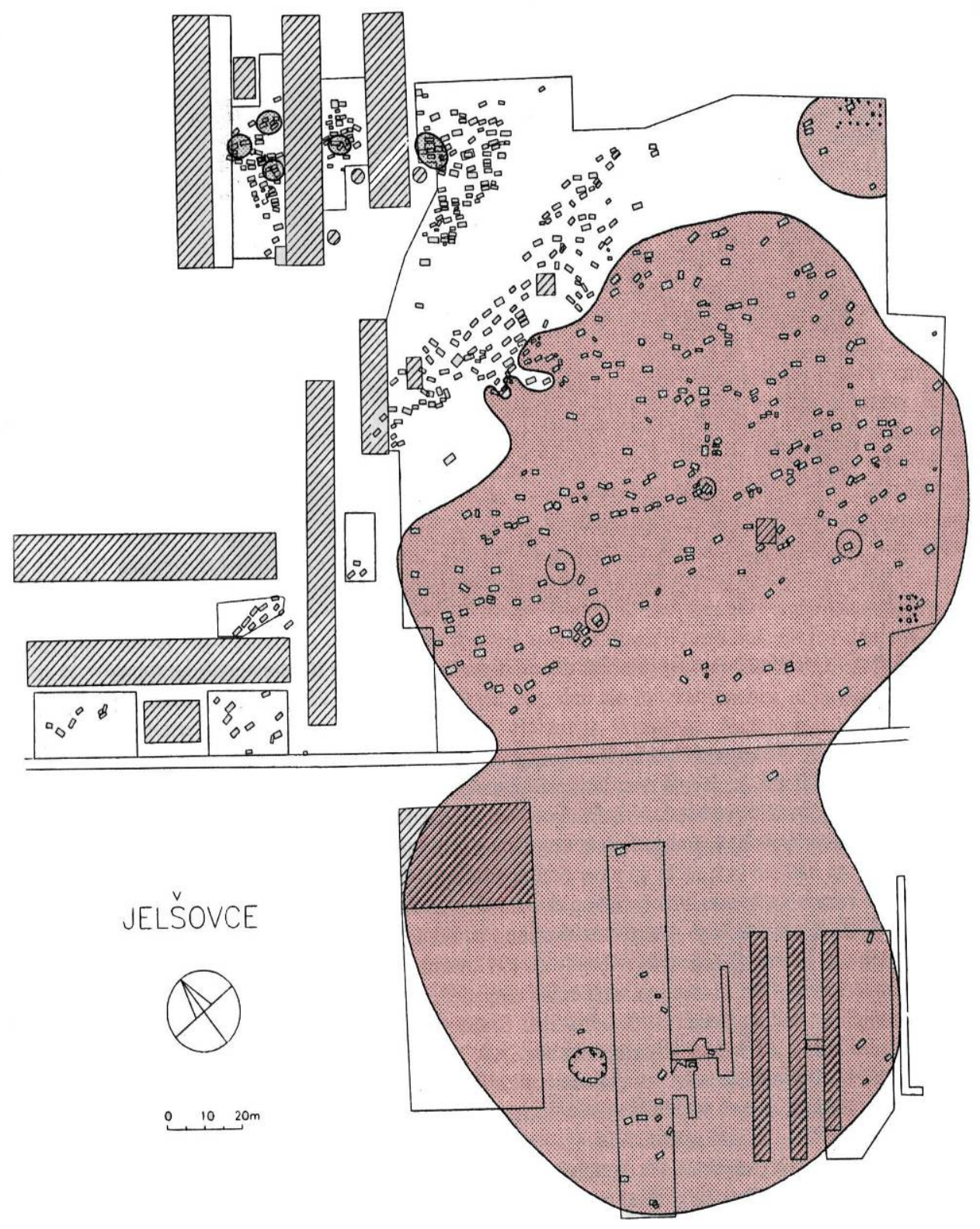

Die Abbildung zeigt das Areal des ehemaligen LPG-Wirtschaftshofes sowie die Grabungsflächen und Gräber in diesem Bereich. Rot markiert sind die Areale, in denen sich mad'arovcezeitliche Gräber befinden.

schraffierte Bereiche: umrandete Bereiche: kleine Rechtecke:
Gebäude

Ausgrabungsflächen

Grabgruben 
Tab. 2 Liste aller Kinder der Mad'arovce-Kultur von Jelšovce nach Grabnummern sortiert

\begin{tabular}{|c|c|c|c|c|c|c|c|}
\hline & $\begin{array}{l}\text { Grab- } \\
\text { Nr. }\end{array}$ & $\begin{array}{l}\text { Alters- } \\
\text { klasse }\end{array}$ & Alter & & $\begin{array}{l}\text { Grab- } \\
\text { Nr. }\end{array}$ & \begin{tabular}{|l} 
Alters- \\
klasse
\end{tabular} & Alter \\
\hline 1 & $6 \mathrm{~B}$ & Ia & 0,5-1 J. & 41 & $173 \mathrm{~B}$ & Ia-Ib & 1-4 J. \\
\hline 2 & $6 \mathrm{C}$ & (Ib-) II & (5-) 6-7 (-8) J. & 42 & 212 & II & 9,5-12 J. \\
\hline 3 & $6 \mathrm{D}$ & $\mathrm{Ib}$ & $2,5-4(-5) \mathrm{J}$. & 43 & 220 & II & 9,5-12,5 J. \\
\hline 4 & $6 \mathrm{E}$ & Ib-II & 4-6,5 J. & 44 & 221 & II & $6-8 \mathrm{~J}$. \\
\hline 5 & $6 \mathrm{~F}$ & II & $6-12 \mathrm{~J}$. & 45 & $224 \mathrm{~A}$ & II & 9,5-11 J. \\
\hline 6 & $6 \mathrm{G}$ & II- (Juv.) & (8-) 11-14 (-16) J. & 46 & $228 \mathrm{~B}$ & II & $6-8 \mathrm{~J}$. \\
\hline 7 & 15 & Ia & 10-16 Mon. & 47 & 241 & $\mathrm{Ib}$ & 3-5 J. \\
\hline 8 & 19 & II & $10-12 \mathrm{~J}$. & 48 & 253 & II & $12-13(-14) \mathrm{J}$. \\
\hline 9 & 26 & Ia & 2-6 Mon. & 49 & 254 & II & 7-9-10 J. \\
\hline 10 & 31 & Ia & 12-16 Mon. & 50 & 271 & Ib-II & 5-7 J. \\
\hline 11 & 41 & $\mathrm{Ib}$ & 2,5-4 J. & 51 & 275 & $\mathrm{Ib}$ & 4,5-6 J. \\
\hline 12 & $65 \mathrm{~A}$ & $\mathrm{Ib}$ & 3-4 J. & 52 & $279 \mathrm{~A}$ & II & 7-9 J. \\
\hline 13 & 72 & Ia (-Ib) & $1,5-2(-2,5) \mathrm{J}$. & 53 & $279 \mathrm{~B}$ & (Ib-) II & 5-7 J. \\
\hline 14 & 81 & Ia-Ib & 1,5-2,5 J. & 54 & 281 & II & 9,5-12 J. \\
\hline 15 & 83 & Ib (-II) & 5,5-6 (-7) J. & 55 & 299 & II & 9-10,5 J. \\
\hline 16 & 84 & II (-Juv.) & $12-14(-16) \mathrm{J}$ & 56 & $300 \mathrm{~A}$ & $\mathrm{Ib}$ & $2-2,5(-3) \mathrm{J}$ \\
\hline 17 & 86 & $\mathrm{Ib}$ & 3-5 J. & 57 & $300 \mathrm{~B}$ & Ia & 3-9 Mon. \\
\hline 18 & $88 \mathrm{~A}$ & $\mathrm{Ib}$ & 2,5-4,5 J. & 58 & $300 \mathrm{C}$ & $\mathrm{Ib}$ & 3-4 J. \\
\hline 19 & $88 \mathrm{~B}$ & II & $8-10 \mathrm{~J}$. & 59 & 301 & $\mathrm{Ib}$ & 4,5-5,5 (-6) J. \\
\hline 20 & 95 & $\mathrm{Ib}$ & 3,5-5,5 J. & 60 & 318 & II & 7,5-10 J. \\
\hline 21 & 97 & Ib-II & 5-9 J. & 61 & 331 & $\mathrm{Ib}$ & $2,5-3,5 \mathrm{~J}$. \\
\hline 22 & 99 & II & 6-8 J. & 62 & 332 & $\mathrm{Ib}$ & 3-4 J. \\
\hline 23 & 102 & II & 9-10,5 J. & 63 & 334 & II & 12-14 J. \\
\hline 24 & $103 \mathrm{~B}$ & II & 9-13 J. & 64 & $336 \mathrm{~B}$ & $\mathrm{Ib}$ & $2-4 \mathrm{~J}$. \\
\hline 25 & 105 & $\mathrm{Ib}$ & 3-5 J. & 65 & $337 \mathrm{~A}$ & $\mathrm{Ib}$ & 4-6 J. \\
\hline 26 & 113 & II & 9,5-12 J. & 66 & $337 \mathrm{~B}$ & Ia & 0,5-1 J. \\
\hline 27 & 118 & $\mathrm{Ib}$ & 3,5-4,5 J. & 67 & 338 & Ia-Ib & 18-26 Mon. \\
\hline 28 & 120 & $\mathrm{Ib}$ & $2,5-3,5 \mathrm{~J}$. & 68 & 339 & $\mathrm{Ib}$ & 2,5-4,5 J. \\
\hline 29 & 126 & II & 9-11 J. & 69 & 340 & Ia & $1-2 \mathrm{~J}$. \\
\hline 30 & $134 \mathrm{~B}$ & II & 8-11 J. & 70 & 346 & Ia-Ib & $1,5-2,5(-3,5) \mathrm{J}$. \\
\hline 31 & $135 \mathrm{~A}$ & II & 9-11 J. & 71 & 350 & II & 7-9 J. \\
\hline 32 & $140 \mathrm{~B}$ & II & 6-12 J. & 72 & 376 & II & $6-8 \mathrm{~J}$. \\
\hline 33 & $140 \mathrm{C}$ & $\mathrm{Ib}$ & 2-2,5 (-3) J. & 73 & $561 \mathrm{~A}$ & $\mathrm{Ib}$ & 2-4 J. \\
\hline 34 & 155 & II & 8-10 (-11) J. & 74 & $561 \mathrm{~B}$ & $\mathrm{Ib}$ & $2-5 \mathrm{~J}$. \\
\hline 35 & $158 \mathrm{~A}$ & II & $7-10 \mathrm{~J}$. & 75 & $561 \mathrm{C}$ & Ib-II & 4-8 J. \\
\hline 36 & $158 \mathrm{~B}$ & II & $6-12 \mathrm{~J}$. & 76 & 566 & (Ib-) II & $(5,5-)$ 6-6,5 (-7) J. \\
\hline 37 & 162 & Ia & 0,5-1 J. & 77 & $604 \mathrm{~B}$ & II & $11-14 \mathrm{~J}$ \\
\hline 38 & 164 & II & 7-6 J. & 78 & $616 \mathrm{~B}$ & II & 9-11 J. \\
\hline 39 & 169 & II & 6-7 (-8) J. & 79 & 626 & II & 5-8 J. \\
\hline 40 & $173 \mathrm{~A}$ & II & 6-8 J. & & & & \\
\hline
\end{tabular}




\section{$\underline{\text { II.2 Methoden }}$}

\section{II.2.1 Paläopathologische Untersuchungsmethoden}

\section{II.2.1.1 Makroskopische Untersuchung}

Zu Beginn der Bearbeitung wurde das Skelet ausgelegt. Alle vorhandenen Knochen wurden in einen Skeletbogen eingezeichnet, makroskopisch auf Spuren pathologischer Prozesse hin untersucht. Das Lupenmikroskop wurde zur Differenzierung von postmortalen und intravitalen Veränderungen an Oberflächen eingesetzt. Alle Befunde wurden auf speziellen Befundbögen dokumentiert und beschrieben. Veränderungen des Schädels wurden auch grafisch auf einem Spezialbogen festgehalten (SCHULTZ 1988 a).

Für die Klassifikation der Cribra orbitalia wurden die Kriterien nach Schultz (1988 a) verwandt (s. Anhang). Zahnstatus und Kieferveränderungen wurden in einen dafür vorgesehenen Bogen (SCHULTZ 1988 a) eingetragen. Die Gradeinteilungen von Parodontopathien, Zahnstein, Karies und transversalen Schmelzhypoplasien wurden ebenfalls von Schultz (1988a) übernommen (s. Anhang). Das Entstehungsalter der Schmelzhypoplasien wurde nach Ubelaker (1980) bestimmt. Der Abrasionsgrad wurde nach Brothwell (1981) und Perizonius und Pot (1981) beurteilt. Pathologische Veränderungen wurden fotografisch (farbig, digital - Digitalkamera Casio und Digitalkamera Sony DSC-V1) dokumentiert. Die digital aufgenommenen Bilder wurden zur Markierung der Schnittebene für die Dünnschliffpräparate über einen Laserdrucker (LaserJet 4, Hewlett Packard) schwarz-weiß ausgedruckt.

\section{II.2.1.2 Lupenmikroskopische Untersuchung}

Von einigen besonders interessanten Oberflächenveränderungen wurden Lupenfotos hergestellt (Photomakroskop M 400, Wild/ Heerbrugg, Schweiz). 


\section{II.2.1.3 Endoskopische Untersuchung}

Der Endocranialraum aller vollständig erhaltenen Schädel, die Nasennebenhöhlen, die pneumatischen Räume des Os temporale (Mittelohr, Mastoidzellen) und vereinzelt auch die Markröhren der Langknochen wurden mit einem Stabendoskop der Firma Volpi endoskopiert. Interessante pathologische Befunde wurden fotografisch festgehalten.

\section{II.2.1.4 Röntgenologische Untersuchung}

Makroskopisch veränderte Langknochen sowie Schädeldächer mit außergewöhnlichen Veränderungen wurden geröntgt. Zur Beurteilung des Grades der Pneumatisation des Warzenfortsatzes wurden nicht postmortal eröffnete Processus mastoidei durchleuchtet. Einige typische Beispiele für normale und pathologische Pneumatisation wurden zudem geröntgt.

Zur Darstellung der Harris-Linien wurden alle Tibiae, die mindestens eine vollständige Metaphyse aufweisen, in anterior - posteriorem Strahlengang geröntgt. Es wurden nur diejenigen Linien gewertet, die mindestens ein Drittel der Schaftbreite erreichten (BYERS 1991). Die Errechnung des Entstehungsalters erfolgte nach Wells (1967), Schultz (1978) und Templin (1993). Grundlage der Berechnung sind die Tabellen zur Altersbestimmung an Langknochen von Stloukal und Hanáková (1978).

Alle Röntgenbilder wurden mit dem „Faxitron“ der Firma Hewlett Packard hergestellt. Es handelt sich hierbei um ein Vollschutzröntgengerät, das eine Röntgenstromstärke von 3 mA besitzt und dessen Spannung sich im Bereich von 10 bis 110 kV regeln lässt. Als Filme wurden Structurix NDT 65 von Agfa und Cronex NDT 65 von Du Pont, beide folienlos, verwendet. 


\section{II.2.1.5 Anfertigung von Abgüssen}

Von einer unklaren Impression der Lamina externa eines Os frontale (Grab 228 B) wurde ein Abguss angefertigt.

\section{II.2.1.6 Lichtmikroskopische Untersuchung}

Bei makroskopisch unklarer Diagnose oder zur Bestätigung der makroskopischen Ergebnisse, sowie zur Unterscheidung von intravitalen oder postmortalen Veränderungen wurden mikroskopische Präparate angefertigt, die eine histologische Untersuchung ermöglichen. Dazu mussten Proben aus dem Knochen gesägt werden. Nach der Einbettung der Proben in Epoxidharz (Biodur) wurden ungefärbte Dünnschliffe hergestellt (Verfahren von Schultz und Brandt; SCHUlTz \& DROMMER 1983 und SCHUlTz 1988 b). Die fertigen Schliffe wurden unter einem Lichtmikroskop im einfachen und polarisierten Durchlicht befundet.

\section{II.2.1.7 Rasterelektronenmikroskopische Untersuchung}

Bei ausgewählten Fälle wurden zur Beurteilung der Oberflächen die für die Histologie gesägten Proben vor ihrer Einbettung im Rasterelektronenmikroskop untersucht (Digital Scanning Microscope, DCM 960, Zeiss). Vor der Untersuchung wurden die Proben mit einer dünnen Gold-Palladium-Schicht bedampft. Die Befunde wurden fotografisch dokumentiert.

\section{$\underline{\text { II.2.2 Bestimmung des individuellen Sterbealters }}$}

Die Altersbestimmung wurde anhand der Tabellen mit den Längen der Extremitätenknochen (STLOUKAL \& HANÁKOVÁ 1978, JOHNSTON 1962) und des Zahnentwicklungsstandes sowie des Durchbruchstandes einzelner Zähne (UBELAKER 1980) vorgenommen. Soweit dies möglich war, wurden auch die Schlusszeiten der verschiedenen Epiphysenfugen (Ossifikationsmerkmale) berücksichtigt (nach SzILVÁSSY 1988, in Anlehnung an FEREMBACH et al. 1979). 
Folgende Altersklassen wurden unterschieden:

Fetus: 4. Lunarmonat - reifer Fetus (bis zur Geburt)

Infans I: $\quad$ Geburt - 6 Jahre (bis zum Durchbruch des 1. Molaren)

Ia: Geburt bis 2 Jahre

Ib: 2 bis 6 Jahre

Infans II: 6 bis 14 Jahre (bis spätestens zum Durchbruch des 2. Molaren)

Juvenil: $\quad 14$ bis 18 (bis 20) Jahre (bis zum Schluss von Sphenobasilarfuge und/ oder

Durchbruch des 3. Molaren)

Wenn sich die Altersspanne eines Individuums nicht einer einzigen Altersklasse zuordnen ließ, wurde es anteilig auf die verschiedenen Altersklassen aufgeteilt. Bei den Gräbern 26 und 561, die nicht zur Untersuchung zur Verfügung standen, wurde die Altersbestimmung von Dr. Julius Jakab übernommen.

\section{II.2.3 Aufbau des Katalogs}

Die Befunde wurden für diese Arbeit in einem nach Schlomm (2000) modifizierten und erweiterten Katalogblatt dargestellt. Einer Übersicht über Fundort, Erhalt und Alter eines Individuums folgt die Beschreibung der Befunde an Cranium, Zähnen und Postcranium. Nicht vorhandenen Strukturen werden im Befundteil nicht aufgeführt. In einem Skeletschema sind die vorhandenen Knochen markiert.

Am Ende werden die wichtigen Befunde zusammengefasst aufgelistet. 


\section{Ergebnisse}

\section{III.1 Altersverteilung der Kinder}

Der Anteil der Kinder von der Geburt bis zum 14. Lebensjahr an der Gesamtbevölkerung der untersuchten Population beträgt 23,3 \% ( $\mathrm{n}=79 / 339)$, Juvenile und Erwachsene stellen zusammen 76,7 \% ( $\mathrm{n}=260$ / 339) (Tab. 3 und Abb. 6); Früh- und Neugeborene wurden nicht gefunden (s.u.). Zwischen der Geburt und dem 6. Lebensjahr verstarben 11,5 \% der Kinder (n = 39/ 339). Davon gehören 3,4 \% $(n=11,5 / 339)$ in die Gruppe Infans Ia und 8,1 \% $(n=27,5 /$ 339) in die Gruppe Infans Ib. Zwischen dem 6. und dem 14. Lebensjahr verstarben 11,8 \% ( $n=40 / 339)$ aller Individuen (Tab. 3 und Abb. 7). Vergleicht man die Anteile innerhalb der Kinderpopulation so gehören der Gruppe Infans I 49,4 \% (n= 39/ 79) und der Gruppe Infans II 50,6 \% $(n=40 / 79)$ der Kinder an. Die Gruppe Infans I besteht zu 14,6 \% $(n=11,5 / 79)$ aus Kindern der Untergruppe Infans Ia und zu 34,8 \% $(n=27,5 / 79)$ aus Kindern der Gruppe Infans Ib (Tab. 4 und Abb. 8). Das Fehlen der ganz kleinen Kinder ist möglicherweise durch Siedlungsbestattungen zu erklären (vgl. IV.2.1). Die zugehörige Siedlung wurde im Rahmen einer Rettungsgrabung nur unvollständig ergraben (KUDLÁČEK 1958) (vgl. IV.2.1).

Tab. 3 Altersverteilung alle Individuen der Mad'arovce-Kultur von Jelšovce

\begin{tabular}{|l|l|l|l|}
\hline Altersklasse & $\begin{array}{l}\text { Alter } \\
\text { [Jahre] }\end{array}$ & $\begin{array}{l}\text { Anzahl n } \\
\text { absolut }\end{array}$ & $\begin{array}{l}\text { Anteil in } \\
\text { Prozent [\%] }\end{array}$ \\
\hline Fetus & unreif & 0 & 0 \\
\hline Infans Ia & NB - 2 & 11,5 & 3,4 \\
\hline Infans Ib & $2-6$ & 27,5 & 8,1 \\
\hline Infans II & $6-14$ & 40 & 11,8 \\
\hline $\begin{array}{l}\text { Juvenile und } \\
\text { Erwachsene }\end{array}$ & älter als 14 & 260 & 76,7 \\
\hline Gesamt & & 339 & 100 \\
\hline
\end{tabular}

(NB: neugeboren) 
Tab. 4 Altersverteilung innerhalb der Kinderpopulation der Mad'arovce-Kultur

\begin{tabular}{|l|l|l|l|}
\hline Altersklasse & $\begin{array}{l}\text { Alter } \\
\text { [Jahre] }\end{array}$ & $\begin{array}{l}\text { Anzahl n } \\
\text { absolut }\end{array}$ & $\begin{array}{l}\text { Anteil der } \\
\text { Kinder in [\%] }\end{array}$ \\
\hline Fetus & unreif & 0 & 0 \\
\hline Infans Ia & NB - 2 & 11,5 & 14,6 \\
\hline Infans Ib & $2-6$ & 27,5 & 34,8 \\
\hline Infans II & $6-14$ & 40 & 50,6 \\
\hline Juvenil & $14-18(20)$ & 0 & 0 \\
\hline Gesamt & bis 14 & 79 & 100 \\
\hline
\end{tabular}

(NB: neugeboren)

Abb. 6 Altersverteilung Kinder gegenüber Juvenilen und Erwachsenen in der Mad'arovce-Kultur in Jelšovce

\section{Altersverteilung in der Mad'arovce-Kultur}

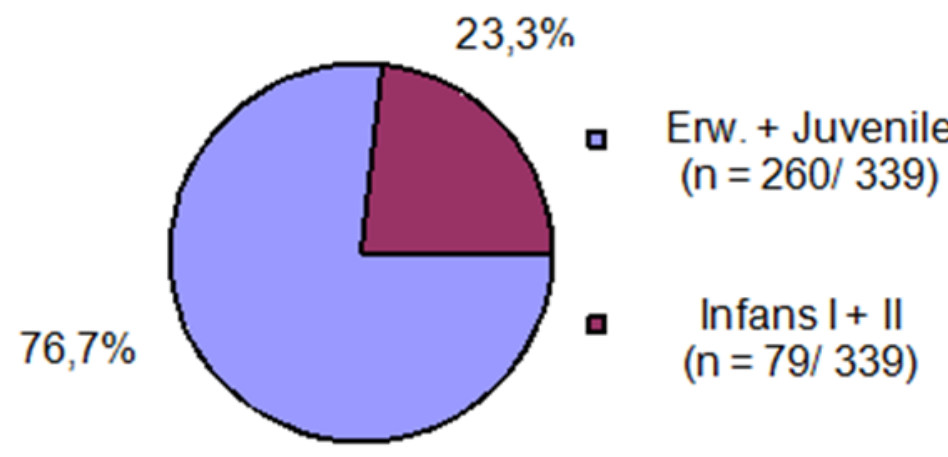

(Erw.: Erwachsene) 
Abb. 7 Altersverteilung der Kinder gegenüber Juvenilen und Erwachsenen in der Mad'arovce-Kultur in Jelšovce, differenziert nach Altersklassen

\section{Gesamtaltersverteilung in der Mad'arovce-Kultur}

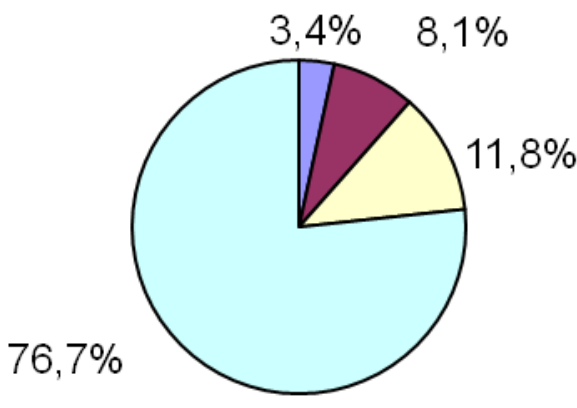

․ Infans la

$(n=11,5 / 339)$

- Infans lb

$(n=27,5 / 339)$

․ Infans II

$(n=40 / 339)$

Erw.+Juv.
$(\mathrm{n}=260 / 339)$

(Erw.: Erwachsene; Juv.: Juvenile)

Abb. 8 Altersverteilung innerhalb der Kinderpopulation der Mad'arovce-Kultur

\section{Altersverteilung in der Kinderpopulation der Mad'arovce- Kultur in Prozent}

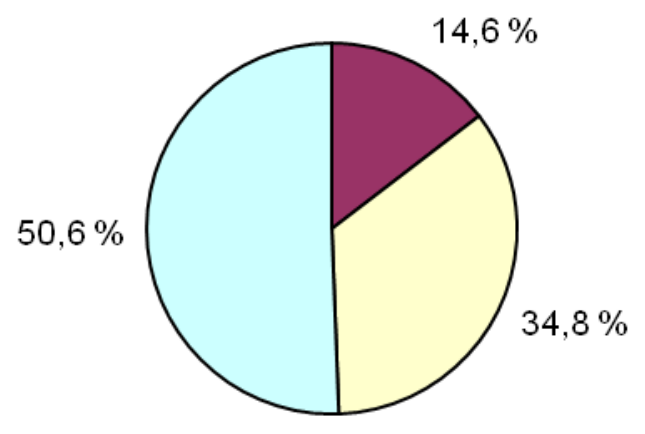

口Infans la $(n=11,5 / 79)$

口Infans Ib $(n=27,5 / 79)$

Infans II ( $\mathrm{n}=40 / 79)$ 


\section{III.2 Spuren pathologischer Prozesse am Cranium}

\section{$\underline{\text { III.2.1 Schädelanomalien und -dysplasien }}$}

Das Kind aus Grab 318 weist eine Deviation der Lambdanaht auf (Tafel 1). Das Os occipitale läuft nach oben hin sehr spitz zu. Rechts befinden sich mehrere Nahtknochen im Bereich der Lambdanaht. Möglicherweise ist die Ursache für diese Besonderheit ein ehemaliger Fontanellenknochen der kleinen Fontanelle, der mit dem Os occipitale synostosiert ist. Insgesamt ist der Schädel des Kindes nicht verformt. Ein Plagiocephalus (= Schiefschädel) besteht nicht. Eine begleitende Hirndrucksymptomatik ist ebenfalls nicht zu finden.

Zeichen einer Hirndrucksymptomatik als Folge eines Hydrocephalus internus finden sich bei 16 von 46 Kindern $(n=16 / 46: 34,8$ \%). Bei diesen Kindern sind die Abdrücke der Hirnwindungen (Impressiones digitatae) in der Lamina interna über die Norm verstärkt (Tafel 1). Bei zwei weiteren Kindern besteht der Verdacht auf einen erhöhten Hirndruck (Gräber 83 und 626).

Medizinischer Hintergrund:

Als Hydrocephalus wird jede Erweiterung der intracraniellen Liquorräume bezeichnet. Aufgrund der starren Verhältnisse des Endocranialraums führt eine Vermehrung der Flüssigkeit zu einem Anstieg des intracraniellen Druckes. Bei einem Hydrocephalus internus besteht eine Liquorzirkulationsstörung. Die Verbindungen zwischen inneren und äußeren Liquorräumen sind verlegt. Ursache für einen Hydrocephalus sind am häufigsten Verklebungen nach Entzündungen (Meningitiden, Meningoencephalitiden), seltener auch Fehlbildungen des Liquorsystems, Tumoren der hinteren Schädelgrube (z.B. Medulloblastome) oder Hirnfehlbildungen. Kraniostenosen beruhen auf der vorzeitigen Synostose von Schädelnähten. Sie führen zu Deformierungen des Schädels. Je nachdem welche Naht sich in welchem Alter schließt, kommt es zu typischen Verformungen des Schädels. Es besteht die Gefahr einer chronischen Erhöhung des intracraniellen Druckes mit cerebralen Funktionsstörungen.

\section{$\underline{\text { III.2.2 Veränderungen der Lamina externa des Schädeldaches }}$}

Makroskopisch sind bei 12,2 \% $(n=5 / 41)$ der Kinder poröse Areale der Ossa parietalia im Bereich der Lambdanaht zu beobachten. Bei einem dieser Kinder (Grab 340) besteht der Ver- 
dacht auf ein Hämangiom des Schädeldachs. Bei den Übrigen ist eine Differenzierung aufgrund des geringen Ausprägungsgrades nicht möglich. Die mikroskopische Untersuchung des porösen Areals bei dem Kind aus Grab 81 zeigt eine stellenweise ausgedünnte, durchbrochene Lamina externa mit einer begleitenden Hypertrophie der Diploë. Die Diploë scheint angedeutet vertikal nach außen zu wachsen. Zeichen einer Knochenmarkshypertrophie in Form von erweiterten Markräumen sind jedoch nicht zu finden. Damit sind zwar nicht alle Merkmale einer Anämie erfüllt (s. III.5.2) (vgl. SCHULTZ 2003), wahrscheinlich handelt es sich trotzdem um ein sehr frühes Stadium einer Anämie (Tafel 2). Bei einem Kind (Grab 72) ist eine feine plattenartige Auflagerung besonders am Os frontale feststellbar, die wahrscheinlich im Rahmen eines Skorbut aufgetreten ist (s. III.5.1).

Das Kind aus Grab 102 weist makroskopisch zwar keine Oberflächenveränderungen des Schädeldaches auf; insgesamt erscheint dieses jedoch verbreitert. Mikroskopisch zeigen sich atypische, tangential zur Oberfläche orientierte Spongiosabälkchen sowie verschmälerte Markräume. Irreguläre und tangential angeordnete, grazile Trabekel der Diploë mit Auflösung der eigentlichen Lamina externa verbunden mit einer Reduktion der Markräume ist typisch für eine Rachitis (ScHULTz 2003). Im vorliegenden Fall ist die Lamina externa unverändert. Zeichen einen aktiven Knochenumbaus sind nicht zu finden. Die Diploëveränderungen weisen auf eine abgeheilte Affektion hin. Differentialdiagnostisch kommen eine ausgeheilte Rachitis oder eine Osteomyelitis in Frage, wobei das komplette Ausheilen einer Osteomyelitis eher unwahrscheinlich ist (Tafel 3) (s. auch III.5.3).

Das Kind aus Grab 253 weist eine Fraktur des Schädeldaches auf, die vermutlich durch einen Schlag auf den Kopf verursacht wurde (Tafeln 4 und 5). Begleitend finden sich Verletzungen der Schädelbasis und des Jochbogens (s. III.2.5, III.2.7 und IV.8.4). Nebenbefundlich besitzt das Kind als epigenetisches Merkmal eine Sutura metopica (Tafel 4).

Das Kind aus Grab 19 zeigt am Os frontale supranasal eine narbige Umgebung um die Öffnung eines Kanals der nach endocranial in den Sulcus sinus sagittalis cranial der Crista frontalis 
führt (Tafel 5). Möglicherweise handelt es sich um die Durchtrittsöffnung einer entzündeten, an untypischer Stelle gelegenen Vene (Emissarvene).

Bei dem Kind aus Grab 228 B findet sich mittig auf dem Os frontale nahe der Kranznaht eine ovale, muldenförmige Läsion mit glatter Begrenzung und wulstigem Rand (Tafel 5). Im Röntgenbild sind außer einer leichten Abplattung der betroffenen Oberfläche keine Auffälligkeiten zu finden. Rasterelektronenmikroskopisch zeigt sich eine gut verheilte, narbig veränderte Oberfläche im Bereich der Mulde (s. IV.8.5).

Medizinischer Hintergrund:

Poröse Oberflächen können ihre Ursache in Mangelerkrankungen (Rachitis, Anämie, Skorbut), Entzündungen (Kopfschwartenentzündung, Osteomyelitis des Schädeldaches) oder Traumen (z.B. Geburtstrauma) haben. Bei ausgeprägter Anämie bildet sich ein so genannter Bürstenschädel. Ein Hämangiom kann zu einer ähnlichen porösen, netz- bis leistenartigen Verdickung des Schädeldaches führen. Frakturen des Schädeldachs entstehen meist durch massive Gewalteinwirkungen auf den Schädel.

\section{$\underline{\text { III.2.3 Veränderungen der Lamina interna des Schädeldaches }}$}

Meningeale Reizungen lassen sich bei zwölf von 45 Kindern ( $n=12$ / 45: 26,7 \%) nachweisen. Aufgrund des Erhaltungszustandes und des Ausprägungsgrades der Veränderungen ist makroskopisch eine Differenzierung der Ursachen nicht sicher möglich. Die für hämorrhagisch bedingte Auflagerungen typischen zungenförmige Platten (SCHULTZ 2001 b und 2003; CARLI-THIELE \& SCHULTZ 2001), sind makroskopisch nur bei zwei Kindern (Gräber 88 und 162) zu erkennen (Tafeln 6 und 49). Mikroskopisch bestätigt sich bei dem Kind aus Grab 162 neben postmortalen Wurzelimpressionen eine hämorrhagisch bedingte Auflagerung (Tafel 6 und 7). Das Kind aus Grab 140 C weist mikroskopisch bei einer perisinusoidal gelegenen Auflagerung in der Fossa cranii posterior ebenfalls hämorrhagisch bedingte Veränderungen als Zeichen eines organisierten epiduralen Hämatoms auf; rasterelektronenmikroskopisch zeigt sich ein gut ausgeheilter Prozess (Tafel 7 und 8). Daraus ergibt sich, dass die meningealen Veränderungen bei drei Kindern ( $\mathrm{n}=3$ / 12: 25 \%) offenbar hämorrhagisch bedingt sind. 
Bei den übrigen Kindern zeigen sich lediglich atypische Gefäßimpressionen, die ebenso entzündlichen wie hämorrhagischen Ursprungs sein könnten. Diese atypischen Gefäßimpressionen treten bevorzugt in den Impressiones digitatae sowohl an den Parietalia als auch an den Temporalia und Occipitalia auf.

Das Kind aus Grab 19 zeigt eine starke Zerstörung der Lamina interna. Mikroskopisch erweisen sich die Veränderungen als postmortal entstanden. Krankhafte intravitale Veränderungen sind nicht zu erkennen.

Bei einem Kind (Grab 212) besteht der Verdacht auf eine tuberkulöse Meningitis. Wie die lichtund rasterelektronenmikroskopische Untersuchung ergab, entstanden die in Gruppen stehenden, kleinen Grübchen in der Fossa cranii posterior offenbar durch Druckatrophie. Derartige Grübchen werden bei meningealer Tuberkulose beobachtet und gehen auf den Abdruck der für diese Krankheit charakteristischen Knötchen zurück (Tafel 9 und 10).

Medizinischer Hintergrund:

Pathologische Vorgänge, die ihre Spuren im Schädelinneren hinterlassen, werden unter dem Begriff der meningealen Reizungen zusammengefasst, der nicht nach den Ursachen differenziert. Epidurale und subdurale Hämatome entstehen durch die Ruptur von Meningealgefäßen bzw. von venösen Hirnblutleitern. Hirnhautentzündungen (Meningitiden) werden überwiegend durch Bakterien oder Viren, seltener durch Parasiten oder Pilze verursacht, treten aber auch nichterregerbedingt als so genannte Begleitmeningitiden im Rahmen anderer Erkrankungen (immunologisches Phänomen) auf. Ursächlich können eine hämatogene Streuung der Erreger, eine kontinuierliche Ausbreitung eines entzündlichen Prozesses (Sinusitis / Mastoiditis) der pneumatischen Räume des Schädels, offene Schädel-Hirnverletztungen sowie eine Kopfschwartenentzündung mit Ausbreitung der Erreger über die Diploëvenen ins Schädelinnere sein. Häufig entwickelt sich in der Folge durch Verklebungen im Subarachnoidalraum ein Hydrocephalus (SCHULTZ 1993) (s. III.2.1).

Eine Sonderstellung nehmen die Meningitiden bei Tuberkulose ein. Eine tuberkulöse Meningitis spielt sich an der Schädelbasis und in der hinteren Schädelgrube ab (bevorzugt Befall caudaler Hirnnerven und Schädelbasisarterien). Sie entsteht sekundär durch eine hämatogene Streuung der Tuberkelbakterien. 
III.2.4 Erkrankungen der venösen Hirnblutleiter:

Eine Perisinusitis ist bei 26,2 \% ( $=11 / 42:$ 26,2 \%) der Kinder feststellbar. Bei weiteren drei Kindern ( $n=3 /$ 42: 7,1 \%) besteht diesbezüglich ein Verdacht. Der Befall der Sinus im Einzelnen ist in der folgenden Tabelle (Tab. 5) aufgeschlüsselt. Die Sinus sigmoidei sind mit 34,7 ( $n=8 / 23)$ häufiger als die Sinus transversi mit 6,7 \% $(n=2 / 30)$ von Erkrankungen betroffen. Auffallend sind die Unterschiede zwischen dem Befall des rechten ( $\mathrm{n}=2$ / 17: 11,7 \%) und des linken ( $\mathrm{n}=7 /$ 18: 38,9 \%) Sinus sigmoideus (Tab.5). Nur bei einem Kind ist der Sinus sagittalis superior erkrankt ( $n=1 / 32: 3,1 \%)$.

Tab. 5 Entzündliche Veränderungen der verschiedenen venösen Hirnblutleiter

\begin{tabular}{|c|c|c|c|c|c|c|}
\hline & $\begin{array}{l}\text { erkrank } \\
\text { te } \\
\text { Ind. }\end{array}$ & $\begin{array}{l}\text { erkrank- } \\
\text { te Ind. } \\
\text { in } \%\end{array}$ & $\begin{array}{l}\text { Ind. V.a. } \\
\text { erkrankt }\end{array}$ & $\begin{array}{l}\text { Ind. V.a. } \\
\text { erkrankt } \\
\text { in \% }\end{array}$ & $\begin{array}{l}\text { Ind. V.a. } \\
\text { erkrankt } \\
\& \\
\text { erkrankt }\end{array}$ & $\begin{array}{l}\text { Ind. V.a. } \\
\text { erkrankt } \\
\& \\
\text { erkrankt } \\
\text { in \% }\end{array}$ \\
\hline S. sagittalis sup. & $1 / 32$ & 3,1 & $0 / 32$ & 0 & $1 / 32$ & 3,1 \\
\hline S. transversus & $2 / 30$ & 6,7 & $1 / 30$ & 3,3 & $3 / 30$ & 10 \\
\hline rechts & $2 / 23$ & 8,7 & $1 / 23$ & 4,3 & $3 / 23$ & 13 \\
\hline links & $0 / 20$ & 0 & $0 / 20$ & 0 & $0 / 20$ & 0 \\
\hline S. sigmoideus & $8 / 23$ & 34,7 & $2 / 23$ & 8,7 & $10 / 23$ & 43,5 \\
\hline rechts & $2 / 17$ & 11,7 & $1 / 17$ & 5,9 & $3 / 17$ & 17,6 \\
\hline Links & $7 / 18$ & 38,9 & $2 / 18$ & 11 & $9 / 18$ & 50 \\
\hline
\end{tabular}

(Ind.: Individuen, V.a.: Verdacht auf)

Das Kind aus Grab 318 zeigt im linken Sinus sigmoideus ein rundes Foramen mit gezacktem, unregelmäßigem Rand (Tafel 11). Die Umgebung dieses Foramens besitzt eine netzartige, stark feinporöse Oberfläche. Makroskopisch und endoskopisch ist nicht sicher festzustellen, ob es sich um eine intravitale Perforation eines Mittelohrprozesses in den Sinus sigmoideus oder eine postmortale Zerstörung des Knochens handelt, da die Oberfläche stark postmortal verfremdet ist. Am Os temporale links ist oberhalb des Meatus acusticus externus ebenfalls 
ein kleines Foramen mit poröser Umgebung zu erkennen (Tafel 11). Auch hier ist nicht sicher zu sagen, ob es sich um ein intravitales Geschehen handelt. In Verbindung mit diesen beiden Veränderungen ist ein perforierter Mittelohrprozess jedoch durchaus denkbar.

Medizinischer Hintergrund:

Es wird zwischen septischen (entzündlichen) und aseptischen Sinusthrombosen und Sinusvenenthrombosen (Brückenvenen) und unterschieden. Aseptische Sinusthrombosen treten vor allem bei Erwachsenen mit Gerinnungsstörungen auf, bei Säuglingen auch in Folge von Ernährungsstörungen bei Mangelernährung (STAEMMLER 1958). Die septischen Sinusthrombosen werden vor allem durch das Übergreifen entzündlicher Prozesse der benachbarten Strukturen verursacht. Meist breiten sich die Prozesse kontinuierlich von den pneumatischen Räumen des Schädels oder der Orbita aus. Otitiden und Mastoiditiden führen vor allem zu Thrombosen des Sinus transversus und des Sinus sigmoideus, während Entzündungen von Nasen- und Nasennebenhöhlen vor allem den Sinus cavernosus betreffen. Bei längerem Bestehen kann der Thrombus organisiert und der Sinus rekanalisiert werden. Es bleibt ein abgeflachter, narbig veränderter Sinus zurück.

\section{$\underline{\text { III.2.5 Veränderungen an der Schädelbasis }}$}

Das Kind aus Grab 253 zeigt eine perimortale Fraktur der Schädelbasis, die am Foramen occipitale magnum gelegen ist. Zusammen mit der äußeren Schädelverletzung (s. III.2.2 und III.2.7) ist ein Trauma als Todesursache sehr wahrscheinlich (Tafel 4 und 5) (s. IV.8.4), da auch Spuren einer Heilung nicht auszumachen sind.

Das Kind aus Grab 566 weist am Boden des linken Canalis caroticus netzartige, wulstige Neubildungen auf (Tafel 10).

Medizinischer Hintergrund:

An der Schädelbasis verlaufen fast alle gehirnversorgenden Gefäße sowie die meisten Hirnnerven. Prozesse der Schädelbasis haben vor allem neurologische Symptome zur Folge. Fehlbildungen (z.B. bei Klippel-Feil-Syndrom, s. III.4.5) können zu Einengungen des Hirnstamms führen. Traumatisch bedingte Schädelbasisfrakturen verlaufen durch massive Blutungen oder Gehirnverletzungen häufig tödlich. 


\section{III.2.6 Erkrankungen der Orbita}

Bei 20 von 32 Kindern finden sich Veränderungen des Orbitadaches in Form von Cribra orbitalia, bei weiteren zwei Kindern besteht der Verdacht darauf $(n=20$ / 32: 62,5 \%; $n=2$ / 32: 6,25 \%; $\mathrm{n}=$ 22/ 32: 68,75 \%). Die Verteilung auf die drei Schweregrade (SCHULTZ 1988 a) zeigt, dass bei den Kindern der Mad'arovce-Kultur überwiegend die leichteren Formen auftreten. Bei 60 \% der betroffenen Kinder ( $n=12 / 20$ : 60 \%) tritt Grad I auf. Bei 35 \% der Kinder ( $n=7 / 20: 35 \%)$ ist der Grad II nachweisbar. Nur zwei von 20 Kindern ( $n=2 / 20: 10 \%)$ (Gräber 72 und 340) sind schwer betroffen (Grad III). Mikroskopisch wurden die Orbitadächer von sieben Individuen (Gräber 72, 88 A, 126, 169, 299, 300, 336 B) untersucht; ein rechtes Orbitadach (Grab 72) auch rasterelektronenmikroskopisch. Zeichen einer beginnenden Anämie finden sich bei dem Kind aus Grab 72 in Form einer Ausdünnung der Lamina externa sowie parallelen, senkrecht zur Oberfläche ausgerichteten Spongiosabälkchen (Tafel 12 und 13). Bei diesem Kind sind zusätzlich hämorrhagisch bedingte Veränderungen als Zeichen eines Skorbuts in Form von mehreren Lagen parallel zur Oberfläche orientierten Knochenneubildungen zu erkennen (Tafeln 12 und 13) (s. IV.8.2). Bei dem Kind aus Grab 126 handelt es sich eventuell ebenfalls um eine anämisch bedingte Cribra orbitalia. Hier zeigen sich neben starker postmortaler Verfremdung einzelne angedeutet senkrecht zur Oberfläche orientierte Spongiosabälkchen, die auf eine beginnende Anämie hindeutet. Die Markräume sind dabei aber nicht vergrößert (Tafel 15). Aufgrund erweiterter Markräume besteht bei dem Kind aus Grab 299 differentialdiagnostisch zu einer Entzündung auch der Verdacht einer beginnenden Anämie (Tafel 16). Bei dem Kind aus Grab 336 B sind neben diagenetischen Veränderungen auch entzündlich und hämorrhagisch bedingte Veränderungen festzustellen (Tafel 14). Die Cribra orbitalia des Kindes aus Grab 88 A sind größtenteils postmortal verursacht. Das Orbitadach weist eine Spongiosadystrophie auf, die weder anämisch, noch rachitisch oder hämorrhagisch bedingt ist (Tafel 15). Es besteht der Verdacht auf eine vom Sinus frontalis ausgehende Entzündung (vgl. III.2.9). Bei den Kindern aus den Gräbern 169 und 300 lässt sich 
eine Hypervaskularisation der Orbitadächer nachweisen. Bei dem Kind aus Grab 169 handelt es sich bei gleichzeitig leicht erweiterten Spongiosaräumen und einer atypisch konfigurierten Spongiosa am ehesten um einen entzündlichen Prozess. Bei dem Kind aus Grab 300 ist die Spongiosa nicht sicher pathologisch. Ob die Veränderungen hier entzündlicher oder hämorrhagischer Natur sind, ist nicht sicher zu sagen (Tafel 16).

Auch alle übrigen Wände der Orbita können von pathologischen Veränderungen betroffen sein. Bei sechs von 23 Kindern ist der Orbitaboden porös ( $n=6 / 23:$ 26,1 \%). Bei einem Kind (Grab 72) ist eine Auflagerung nachweisbar.

Medizinischer Hintergrund:

Cribra orbitalia ist die rein morphologische Beschreibung poröser Oberflächen des Orbitadaches. Sie können z.B. entzündlicher, hämorrhagischer, rachitischer, anämischer oder postmortaler, diagenetischer Ursache sein und gelten - wenn sie intravitalen Ursprungs sind - als unspezifische Stressmarker.

\section{$\underline{\text { III.2.7 Veränderungen des Jochbogens }}$}

Das Kind aus Grab 253 weist eine intravitale Sprengung der Naht zwischen Os zygomaticum und Maxilla des rechten Jochbogens auf. Dabei wurde ein kleiner Teil des Os zygomaticum mit abgesprengt (Tafel 4). Diese Verletzung steht offensichtlich im Zusammenhang mit den Verletzungen an Schädelbasis und Schädeldach (s. III.2.2, III.2.5 und IV.8.4).

\section{III.2.8 Erkrankungen der Nasenhöhle}

Bei $40 \%(n=8 / 20)$ der Mad'arovce-Kinder sind die Nasenhöhlen von krankhaften Veränderungen im Sinne einer Rhinitis betroffen. Bei fünf der erkrankten Kinder sind die Oberflächen der lateralen Wände und des Bodens aufgrund der bestehenden Hypervaskularisation bei akuter Entzündung vermehrt porös. Bei drei Kindern ( $n=3 / 20$ : 15 \%) finden sich auch narbig veränderte, wulstige Wandflächen als Zeichen eines chronischen Entzündungsprozesses. Bei vier weiteren Kindern besteht der Verdacht auf eine Rhinitis ( $n=4 / 20: 20 \%)$. Insgesamt 
bestehen nur relativ diskrete Krankheitsspuren, so dass eine granulomatöse Rhinitis ausgeschlossen werden kann.

Die Ductus nasolacrimales sind bei acht von 17 Kindern $(n=8 / 17: 47,1 \%)$ ein- oder beidseits als Zeichen einer Entzündung feinporös oder wulstig verändert. Bei zwei weiteren Kindern ( $\mathrm{n}=2 /$ 17: 11,8 \%) besteht der Verdacht auf eine Entzündung. Bei dem Kind aus Grab 318 besteht eine starke Seitendifferenz in der Weite der Ductus. Der rechte Ductus nasolacrimalis ist im unteren Teil zystisch erweitert, der linke hingegen relativ eng.

Medizinischer Hintergrund:

Eine akute Rhinitis wird von Viren (z.B. Rhino-, Influenza-, Parainfluenza- oder Coronaviren) verursacht. Normalerweise heilt eine Rhinitis nach ein bis zwei Wochen folgenlos ab. Eine bakterielle Superinfektion führt zu einer eitrigen Rhinitis. Komplikationen sind eine Fortleitung der Erreger in Nasennebenhöhlen und Mittelohr.

\section{III.2.9 Erkrankungen der Nasennebenhöhlen}

Bei sechs von 17 Kindern der Mad'arovce-Kultur ( $n=6 / 17: 35,3$ \%) sind Veränderungen im Sinne einer Sinusitis maxillaris nachweisbar (Abb. 9). Bei weiteren drei Kindern besteht der Verdacht auf eine Entzündung der Kieferhöhlen ( $n=3 / 17:$ 17,6 \%). Die chronische Entzündung zeigt sich bei dem Kind aus dem Grab 65 A im Endoskop durch wulstig, vernarbte Oberflächen (Tafel 17). Bei dem Kind aus Grab 334 ist der Boden des Sinus als Zeichen einer akuten Entzündung über die Norm verstärkt porös (Tafel 17). Das Kind aus Grab 337 zeigt als Zeichen einer Entzündung am Boden des rechten Sinus über der Alveole von 16 eine feinporöse, plattenartige Auflagerung.

Die Sinus frontales (Stirnhöhlen) sind bei drei von sieben Kindern stark asymmetrisch ausgebildet. Die Seitendifferenzen sind so ausgeprägt, dass die eine Seite kaum bzw. gar nicht pneumatisch entwickelt, während die andere normal ausgebildet ist. Bei zwei Kindern (Gräber 616 B und 169) zeigt sich an der vorderen Wand des rechten Sinus eine erbs- bzw. reiskorngroße Exostose. Diese Neubildungen repräsentieren offenbar Osteome, die wohl als Folge 
eines chronischen Entzündungsprozesses entstanden sein dürften ( $n=2$ / 7: 28,6 \%) (Abb. 9). Das Kind aus Grab 169 weist im Knochendünnschliff des rechten Orbitadaches und des angrenzenden Os frontale ebenfalls Veränderungen auf, die auf eine Entzündung des Sinus frontalis hinweisen (vgl. III.2.6) (Tafel 15).

Die Cellulae ethmoidales (Siebbeinzellen) sind aufgrund ihrer besonders dünnen Wände nur selten bei archäologischen Funden erhalten. In der Mad'arovce-Kultur sind nur bei vier Kinder Reste der Siebbeinzellen erhalten geblieben. Pathologische Veränderungen lassen sich bei keinem Kind erkennen.

Die Sinus sphenoidales (Keilbeinhöhlen) sind bei sechs Kindern erhalten. Nur bei drei dieser Kinder sind die Sinus auch befundbar. Bei einem Kind (Grab 113) zeigen sich Gefäßimpressionen, die im Rahmen einer Entzündung entstanden sein könnten; die anderen beiden Kinder sind gesund $(\mathrm{n}=1 / 3$ : 33,3 \%) (Abb. 9).

Medizinischer Hintergrund:

Eine akute Sinusitis entsteht durch Fortleitung aus einer akuten Rhinitis. Chronische Sinusitiden entwickeln sich aus einer nicht ausheilenden akuten oder subakuten Sinusitis durch ständige Reizung der Schleimhäute (BOENNINGHAUS \& LENARZ 2001). Die Sinus maxillares (Kieferhöhlen) sind die am häufigsten betroffenen Nasennebenhöhlen, weil die Abflussverhältnisse anatomisch bedingt besonders ungünstig sind. Der Sekretstau fördert die Entzündung und behindert die Abheilung. Komplikationen bei allen Nasennebenhöhlenentzündungen sind, je nach Lokalisation, ein Durchbruch in den Endocranialraum (Meningitis, Abszesse, Sinusvenen-, Sinusthrombosen), die Orbita (Abszesse, Phlegmone) oder die Entwicklung einer Oberkiefer- oder Stirnbeinosteomyelitis (BOENNINGHAUS \& LENARZ 2001).

Die Ergebnisse zeigen, dass ein Zusammenhang zwischen dem Auftreten einer Sinusitis maxillaris und einer Rhinitis besteht (Abb. 10). Bei sechs der neun Kinder ( $n=6 / 9$ : $67 \%$ ) mit Sinusitis maxillaris bzw. Verdacht auf eine diesbezügliche Entzündung besteht auch eine Rhinitis oder ein Verdacht darauf, von den übrigen drei Kindern ist nur ein Kind gesund, bei den anderen beiden Kindern ist die Nasenhöhle nicht befundbar bzw. nicht vorhanden. Um- 
gekehrt gilt, von den zwölf Kindern mit Rhinitis, leiden sechs auch an einer Sinusitis maxillaris ( $n=6 / 12: 50 \%$; einschließlich Verdachtsfälle). Von den übrigen sechs Kindern sind nur bei drei Kindern die Sinus maxillares erhalten geblieben. Also haben nur $25 \%$ der Kinder mit Rhinitis normale Kieferhöhlen ( $\mathrm{n}=3 /$ 12: 25 \%). Zahnwurzelprozesse, die in ihrer Folge eine dentogene Sinusitis maxillaris verursacht haben, finden sich bei den Kindern der Mad'arovceKultur nicht.

Abb. 9 Die Häufigkeiten von Nasennebenhöhlenerkrankungen bei den Kindern der Mad'arovce-Kultur in Jelšovce (in Prozent).

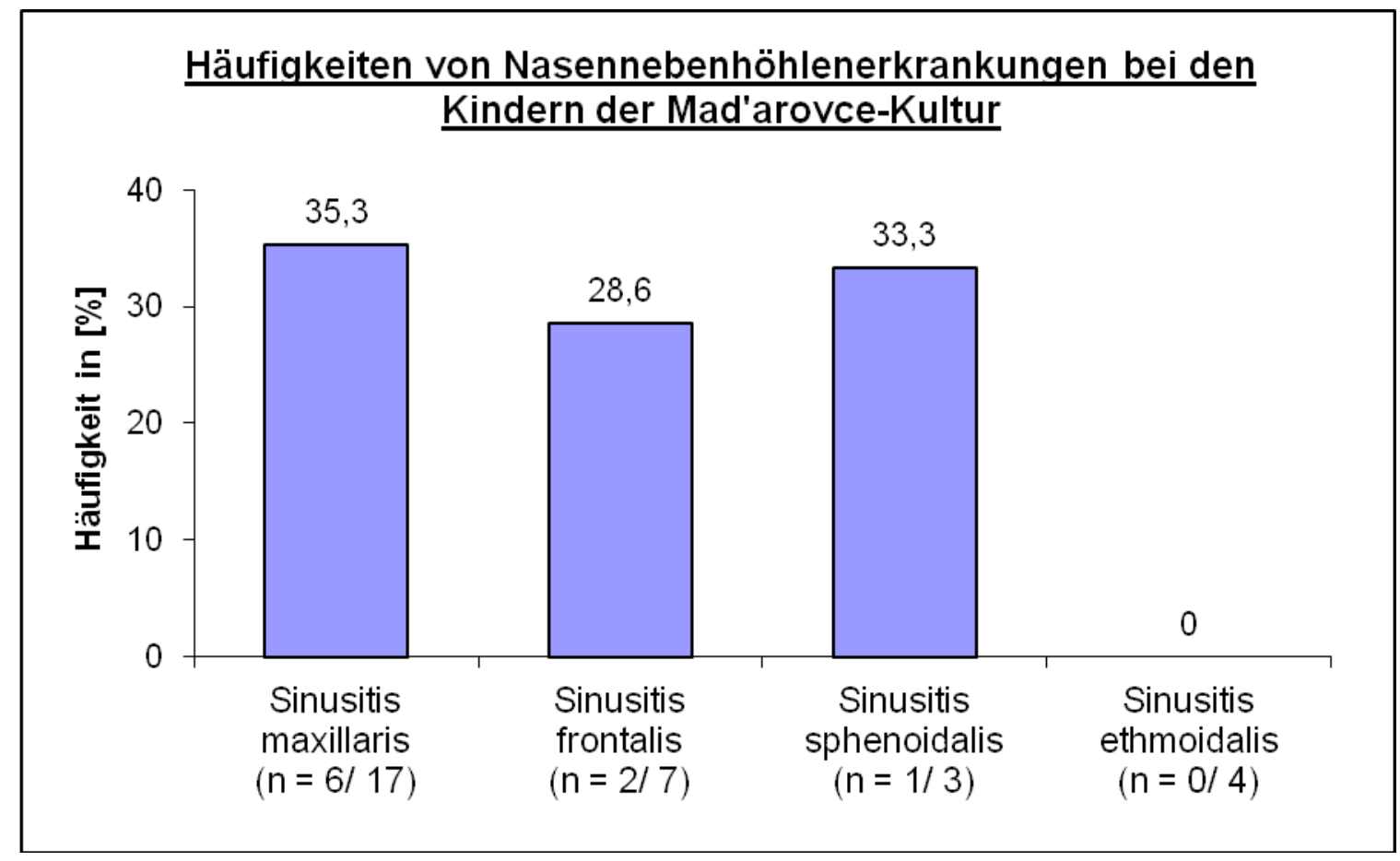


Abb. 10 Die Beziehung zwischen Rhinitis und Sinusitis maxillaris bei den Kindern der

Mad'arovce-Kultur in Jelšovce (unter Berücksichtigung der Verdachtsfälle).

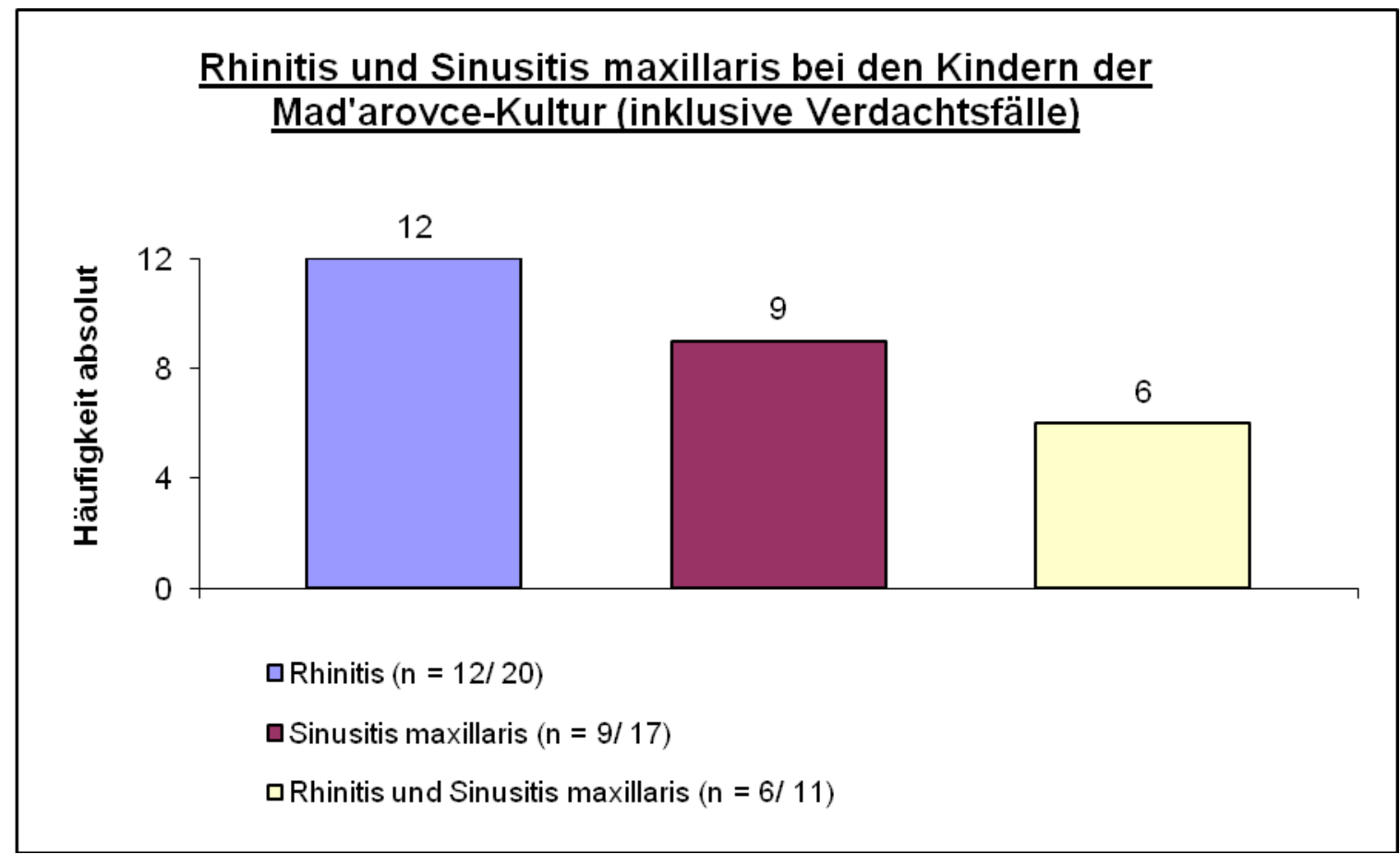

\section{$\underline{\text { III.2.10 Erkrankungen des harten Gaumens }}$}

Zeichen einer Stomatitis weisen zehn von 28 Kindern $(n=10 /$ 28: 35,7 \%) auf. Die knöcherne Gaumenoberfläche ist bei diesen Kindern vor allem im hinteren Anteil über das normale Maß hinaus unregelmäßig porös (Tafel 17). Bei sechs der Kinder ist die Oberfläche zusätzlich wulstig vernarbt. Häufig tritt eine Stomatitis im Rahmen eines Skorbuts auf. Von den zehn an Stomatitis erkrankten Kindern leiden sechs auch an einem Skorbut (n $=6 / 10: 60 \%$ ) (vgl. III.5.1.).

Medizinischer Hintergrund:

Die Entzündung der Schleimhaut des harten Gaumens wird in der Paläopathologie als Stomatitis bezeichnet. Ursächlich sind häufig Erkrankungen der Zähne oder des Zahnhalteapparates. Die Mundhygiene spielt dabei eine wichtige Rolle. Erkrankungen, die ebenfalls zu einer Stomatitis führen können, sind u.a. Skorbut, Agranulozytose, akute Leukämien, Diabetes mellitus, Pilz- und Virusinfektionen. 


\section{$\underline{\text { III.2.11 Erkrankungen des Mittelohres und des Warzenfortsatzes }}$}

Eine Otitis media (Mittelohrentzündung) lässt sich bei drei von 24 Kindern $(n=3 / 24:$ 12,5 \%) nachweisen, bei drei weiteren Kindern besteht der Verdacht auf eine abgelaufene Entzündung. Nur bei einem Kind tritt eine Entzündung beidseits auf (Grab 241); bei drei Kindern ist die Gegenseite gesund; bei zwei Kindern fehlt die andere Seite bzw. ist nicht befundbar.

Endoskopisch zeigt das Promontorium bei einer Otitis media narbige Einziehungen und Wulstungen sowie eine vermehrte Porosität der Oberfläche (Tafel 18).

Medizinischer Hintergrund:

Akute Mittelohrentzündungen entstehen meist durch Fortleitung von Entzündungen über die Tuba auditiva aus dem Nasenrachenraum. Seltener sind Trommelfellperforationen oder ein hämatogener Befall im Rahmen von Viruserkrankungen ursächlich. Bei Säuglingen sind Mittelohrentzündungen häufig. Sie werden durch eine relativ kurze und weite Tuba auditiva begünstigt. Meist treten sie nach viralen Infekten durch bakterielle Superinfektionen (vor allem Pneumokokken und Haemophilus influenzae) auf (KOSENOV 1973, BOENNINGHAUS \& LENARZ 2001).

Eine Mastoiditis ist nur bei einem von 34 Kindern $(n=1 / 33: 3 \%)$ nachzuweisen (Grab 241). Der linke Processus mastoideus dieses Kindes weist in den retrosinuösen Zellen entzündliche Veränderungen auf, ist dabei aber altersentsprechend normal pneumatisiert. Dieses Kind litt ebenfalls an einer beidseitigen Mittelohrentzündung.

Bei sechs von 33 Kindern ( $n=6$ / 33: 18,2 \%) sind Pneumatisationshemmungen zu beobachten (Tafel 11). Bei vier Kindern ist die Gegenseite jeweils gesund, bei einem Kind sind beide Seiten betroffen, bei einem weiteren Kind ist nur eine Seite vorhanden. Möglicherweise stehen diese Verzögerungen des Pneumatisationsprozesses mit abgelaufenen Mittelohrentzündungen in kausalem Zusammenhang. Allerdings sind nur bei dem Kind aus Grab 300 entzündliche Veränderungen des Mittelohres und eine Hemmung der Pneumatisation gemeinsam zu beobachten. 
Medizinischer Hintergrund:

Breitet sich die Entzündungen des Mittelohres in die Cellulae mastoideae aus, sprich man von einer Mastoiditis. Es kommt zu eitrigen Einschmelzungen der knöchernen Zellen des pneumatisierten Warzenfortsatzes. Das Auftreten einer Mastoiditis ist von der Pneumatisation des Warzenfortsatzes und damit auch vom Alter des Kindes abhängig. Die Abheilung wird durch die stark eingeschränkten Abflussmöglichkeiten behindert. Im günstigsten Fall bricht ein Abszess unter das Periost und nach außen durch. Schwerwiegende Komplikationen sind der Einbruch in den Endocranialraum und daraus folgend Sepsis, epidurale Abszesse, Sinusvenen- und Sinusthrombosen, Meningitiden, Meningoencephalitiden oder Hirnabszesse (BOENNINGHAUS \& LENARZ 2001). Entzündliche Prozesse des Mittelohres oder anhaltende Tubenventilationsstörungen besonders in früher Kindheit können zu einer Hemmung oder Verzögerung der Pneumatisation führen, da eine normale Pneumatisation abhängig von einer normalen Schleimhautfunktion und einer guten Belüftung des Mittelohres ist (WITTMAACK 1918, BOENNINGHAUS \& LENARZ 2001).

\section{III.3 Spuren pathologischer Prozesse an Kiefer und Zähnen}

\section{III.3.1 Karies}

Karies kommt bei drei von 49 Kindern vor $(n=3 / 49:$ 6,1 \%) (Abb. 11). Insgesamt sind vier von 509 Zähnen (n= 4/ 509: 0,79 \%) betroffen. Alle Kinder sind der Altersklasse Infans II zugeordnet. Karies tritt lediglich bei Milchzähnen (Milchmolare) mit den Schweregraden I bis III auf (s. Anhang, SchUltz 1988 a). Bei dem Kind aus Grab 616 B sind zwei benachbarte Zähne an der Kontaktfläche betroffen (64 Krone distal, 65 Krone mesial) (Tafel 19). Der Zahn 75 des Kindes aus Grab 83 weist zwei kariöse Läsionen auf, die größere ist occlusal, die kleinere buccal gelegen (Tafel 19). Bei dem Kind aus Grab 318 sind Hals und Wurzel des Zahnes 75 an der mesialen Seite betroffen. Apikale Abszesse sind auf dem Boden der kariösen Läsionen nicht entstanden.

Medizinischer Hintergrund:

Karies wird durch die Erweichung der Zahnhartsubstanzen (Schmelz und Dentin) bedingt. Erster Schritt ist die Entkalkung von Zahnschmelz durch Milchsäure, die beim Abbau von Zuckern durch Bakterien im aufgelagerten 
Zahnbelag (Plaque) entsteht. Kariöse Defekte entstehen, wenn sich der Prozess bis in das Dentin fortsetzt. An Zahnhälsen und in Fissuren ist der Schmelz etwas dünner; folglich sind diese Regionen besonders kariesanfällig (Locus minoris resistentiae). Die kariösen Läsionen können auf den Schmelz beschränkt sein oder bis weit in die Dentinschicht hinein reichen und zur Eröffnung der Pulpahöhle führen. Folgen einer Eröffnung der Pulpahöhle sind Pulpitis, apikaler Prozess und letztendlich der Zahnverlust. Ähnliche Läsionen können auch durch starke Abrasion mit Eröffnung der Pulpahöhle oder postmortal z.B. durch das Einwirken von Huminsäuren entstehen. Dies kann zu Verwechslungen führen.

\section{III.3.2 Zahnstein}

Zahnstein lässt sich bei 27 von 48 Kindern nachweisen ( $\mathrm{n}=$ 27/ 48: 56,3 \%) (Abb. 11) (Tafel 19 und 20). Die Häufigkeit ist deutlich altersabhängig und mit zunehmendem Alter ansteigend (Abb. 12). In der Altersklasse Ia ist nur eins von sechs Kindern $(n=1 / 6: 16,7 \%)$ betroffen, in der Gruppe Infans Ib sind es sieben von $17(\mathrm{n}=7 /$ 17: 41,2 \%), in der Gruppe Infans II 19 von 25 Kindern ( $\mathrm{n}=$ 19/ 25: 76,0 \%). Insgesamt ist Zahnstein an 153 von 509 Zähnen nachweisbar ( $\mathrm{n}=$ 153/ 509: 30,1 \%). Die Mad'arovce-Kinder sind vom Ausmaß her relativ wenig befallen, überwiegend tritt Grad I auf (Grad I: n = 25/ 27: 92,6\%) (s. Anhang, SCHULTZ 1988 a). Diese Beobachtung ist möglicherweise durch einen postmortalen Zahnsteinverlust mitbegründet, z.B. ist ein Abplatzen der Belege während der Bergung und anschließenden Säuberung denkbar.

Medizinischer Hintergrund:

Sehr häufig entsteht Zahnstein bei ungenügender Mundhygiene. Weiche, bakterielle Zahnbeläge werden bei Übersättigung der Beläge mit anorganischen Mineralien zu harten, den Oberflächen aufgelagerten, anhaftenden Belägen umgewandelt. Sie entstehen bevorzugt in der Nähe der Ausführungsgänge der großen Speicheldrüsen (lingual an den unteren Schneidezähnen; buccal an den oberen 1. und 2. Molaren). Die im Speichel enthaltenen Glykoproteine begünstigen ihre Entstehung, weil sie als Substrat für die Bakterien dienen. Aufgrund der irritierenden Wirkung von Zahnsteinbelägen auf das Zahnfleisch begünstigen sie die Entstehung von Parodontopathien. 


\section{III.3.3 Parodontopathien}

Parodontopathien kommen bei 63,4 \% der Kinder vor $(n=26 / 41:$ 63,4 \%). Die Hälfte dieser Kinder ( $\mathrm{n}=13 / 41: 31,7 \%$ ) weisen auch entzündliche Veränderungen (Parodontitis) auf (Abb. 11) (Tafel 19). Alle Kinder, die an einer Parodontitis leiden, sind ebenfalls an Parodontose erkrankt.

Es zeigen sich Unterschiede in der Altersverteilung:

$\begin{array}{llll}\text { Parodontose: Infans Ia } & \text { 0/ } 4 \text { (0\%), } & \text { Ib 9/ } 14(64,3 \%), & \text { II 17/ } 22 \text { (77,3 \%) } \\ \text { Parodontitis: Infans Ia } & \text { 0/ } 4 \text { (0\%), } & \text { Ib 4/ } 14(28,6 \%), & \text { II } 9 / 22(40,9 \%)\end{array}$

Mit zunehmendem Alter nimmt die Erkrankungshäufigkeit sowohl von Parodontose als auch von Parodontitis zu. Die Parodontose tritt deutlich häufiger auf als die Parodontitis (Abb. 11 und 13).

Medizinischer Hintergrund:

Parodontopathien sind Erkrankungen von Zahnfleisch, Zahnhalteapparat und Alveolarknochen. Als Ursachen werden bakterielle Plaque und eine unzureichende Mundhygiene verantwortlich gemacht. Zahnsteinbefall, Karies, Zahn- und Kieferfehlbildungen begünstigen ebenfalls die Entstehung. Parodontose ist durch den nichtentzündlichen, gleichmäßigen Rückgang des Alveolarknochens mit Lockerung der Zähne gekennzeichnet. Am mazerierten Knochen sind die Ränder der Alveolen glatt begrenzt. Eine Parodontitis geht mit der Bildung von eitrigen Zahnfleischtaschen und entzündlichen Veränderungen des Alveolarknochens einher. Sie ist durch einen aggressiven Knochenschwund gekennzeichnet. Am mazerierten Knochen zeigen sich unregelmäßig, zackig, riefig begrenzte Ränder der Alveolen. Reaktive Knochenneubildungen sind möglich. Bei dem Verständnis von Parodontose und Parodontitis gibt es Unterschiede zwischen Paläopathologen und Klinikern. Der Kliniker kann beide im Prinzip nicht voneinander trennen. Der Paläopathologe kann in der Regel zwischen entzündlichen und nicht-entzündlichen Veränderungen am Knochen unterscheiden. Die Begriffe beschreiben in der Paläopathologie also nicht das klinische Erscheinungsbild, sondern den morphologischen Zustand am mazerierten Knochen (s. SCHULTZ 1988 a). 


\section{$\underline{\text { III.3.4.Beziehung zwischen Zahnstein und Parodontopathien }}$}

Die enge Beziehung zwischen dem Befall von Zahnstein und Parodontopathien zeigt sich auch bei den Kindern der Mad'arovce-Kultur. Von 41 Kindern leiden 20 ( $n=20 / 41$ : 48 \%) sowohl an Zahnstein als auch an Parodontopathien (Abb. 15). Nur etwa ein Viertel der Kinder ( $n=10 / 41: 24,4 \%$ ) leidet weder an der einen noch an der anderen Erkrankung. Von den 27 an Zahnstein leidenden Kindern, ist bei 25 der Alveolarknochen beurteilbar. Umgekehrt sind von den 26 an Parodontopathien erkrankten Kindern bei ebenfalls 25 Kindern die Zähne zur Beurteilung des Zahnsteinbefalls erhalten. Von diesen 25 Kindern, bei denen sowohl Zahnsteinbefall als auch Parodontopathien beurteilbar sind, leiden $80 \%$ ( $\mathrm{n}=20$ Kinder) an beiden Erkrankungen (Abb.15).

\section{$\underline{\text { III.3.5 Transversale Schmelzhypoplasien }}$}

Transversale Schmelzhypoplasien kommen bei 31 von 51 Kindern ( $\mathrm{n}=31$ / 51: 60,8 \%) vor (Abb. 11, Tafel 20). Wie Tabelle 6 zeigt, nimmt die Häufigkeit des Auftretens mit dem Alter zu (Abb. 14). Die meisten transversalen Schmelzhypoplasien sind im Alter zwischen eineinhalb und vier Jahren entstanden. Bei keinem Kind sind die Milchzähne betroffen.

Tab. 6 Altersverteilung für transversale Schmelzhypoplasien

\begin{tabular}{|l|l|l|}
\hline Altersstufe & Häufigkeit absolut & Häufigkeit in \% \\
\hline Infans Ia & 1 von 7 & 14,3 \\
\hline Infans Ib & 10 von 19 & 52,6 \\
\hline Infans II & 20 von 25 & 80,0 \\
\hline
\end{tabular}

Etwa 50 \% aller bleibenden Zähne weisen transversale Schmelzhypoplasien auf ( $\mathrm{n}=177 / 355$ : 49,9 \%). Am häufigsten sind die ersten Incisivi und die Canini betroffen (66 \% bzw. 60 \%), gefolgt von zweiten Incisivi und ersten Molaren (56 \% bzw. 54 \%). Am seltensten sind 
transversale Schmelzhypoplasien bei den zweiten Prämolaren und zweiten Molaren zu beobachten (26 \% bzw. 32 \%). Es treten die Schweregrade I bis IV auf (s. Anhang, ScHULTZ 1988 a). Überwiegend kommen jedoch die Grade I und II vor. Eine punktförmige Schmelzhypoplasie zeigt nur ein Kind (Grab 31, Zahn 84).

Medizinischer Hintergrund:

Transversale Schmelzhypoplasien sind Zeichen einer Wachstumsstörung. Die querverlaufenden, rillenförmigen Vertiefungen oder wulstigen Vorwölbungen entstehen bei Störungen des Calciumstoffwechsels oder aufgrund eines Proteinmangels bei Mangelernährung oder schwerer Erkrankung während der Zahnentwicklung. Sie bleiben zeitlebens erhalten und verschwinden nur wenn der Zahnschmelz abgetragen wird (MC HENRY \& SCHULZ 1976). Überwiegend sind die bleibenden Zähne betroffen. Wenn auch Milchzähnen betroffen sind, weist dies auf eine Minderversorgung des Feten während der Schwangerschaft und eine hochgradige Mangelernährung der Schwangeren hin (KÜNZEL \& TOMAN 1985).

Andere Formen von Schmelzhypoplasien haben vielfältige Ursachen und treten sehr viel seltener als transversale Schmelzhypoplasien auf. Disseminiert auftretende, punktförmige Schmelzhypoplasien können genetisch bedingt sein oder lokal durch Traumata entstehen.

\section{III.3.6 Transversale Zementhypoplasien}

Transversale Zementhypoplasien sind den transversalen Schmelzhypoplasien ähnliche Veränderungen. Sie treten im Zahnzement der Zahnwurzeln auf und entstehen aufgrund der späteren Bildung der Zahnwurzeln bei etwas älteren Kindern. Die Pathophysiologie ist die gleiche wie bei transversalen Schmelzhypoplasien. Bei drei Kindern (Gräber 102, 158, 212) konnten transversale Zementhypoplasien nachgewiesen werden, die im Alter von $6 \mathrm{~J} . \pm 24 \mathrm{M}$. bis $10 \mathrm{~J}$. \pm 30 M. entstanden sind (Tafel 20).

\section{$\underline{\text { III.3.7 Veränderungen an Maxilla und Mandibula bei Skorbut }}$}

Skorbuttypische Veränderungen treten am Schädel häufig an Alveolarknochen und Unterkieferästen in Form von porösen Auflagerungen auf (vgl. III.5.1). Poröse Auflagerungen an den 
Rändern der Alveolen finden (Tafel 50) sich bei 35,9 \% der Kinder ( $\mathrm{n}=14 /$ 39: 35,9 \%), bei weiteren 5,1\% ( $\mathrm{n}=2 /$ 39: 5,1 \%) besteht der Verdacht auf Auflagerungen. Porös ausgekleidete Alveolen treten bei sechs von 41 Individuen auf ( $n=6 / 41:$ 14,6 \%). Nur ein Kind (Grab 65 A) zeigt subperiostale Auflagerungen an den Innenseiten der Unterkieferäste.

Abb. 11 Häufigkeiten von Zahnerkrankungen bei den Kindern der Mad'arovce-Kultur in Jelšovce in Prozent

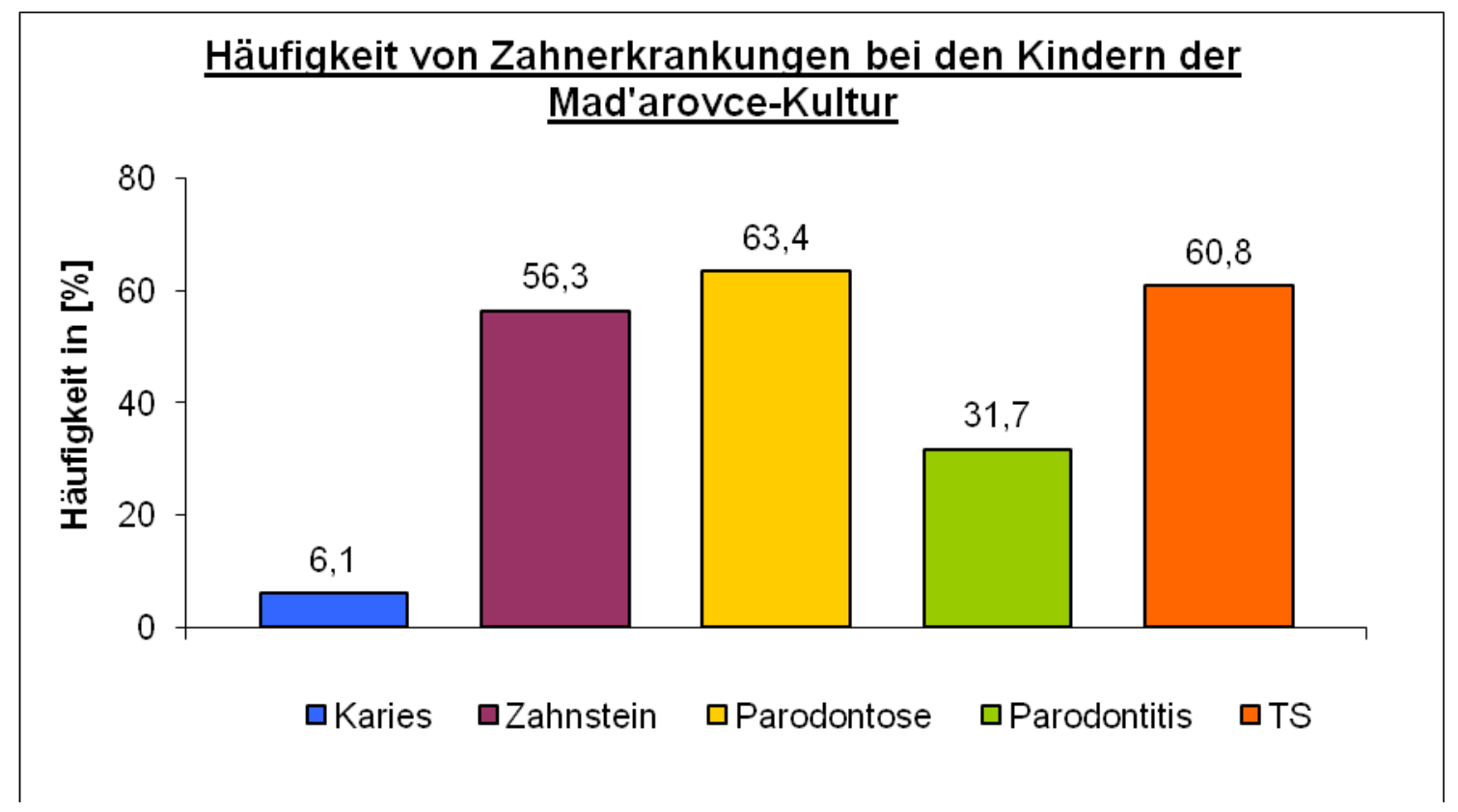

\begin{tabular}{|l|l|l|l|l|l|}
\hline & Karies & Zahnstein & Parodontose & Parodontitis & TS \\
\hline krank absolut & $\mathrm{n}=3 / 49$ & $\mathrm{n}=27 / 48$ & $\mathrm{n}=26 / 41$ & $\mathrm{n}=13 / 41$ & $\mathrm{n}=31 / 51$ \\
\hline krank in [\%] & 6,1 & 56,3 & 63,4 & 31,7 & 60,8 \\
\hline
\end{tabular}

(TS: transversale Schmelzhypoplasien) 
Abb. 12 Verteilung von Zahnstein bei den Kindern der Mad'arovce-Kultur in Jelšovce in den einzelnen Altersklassen

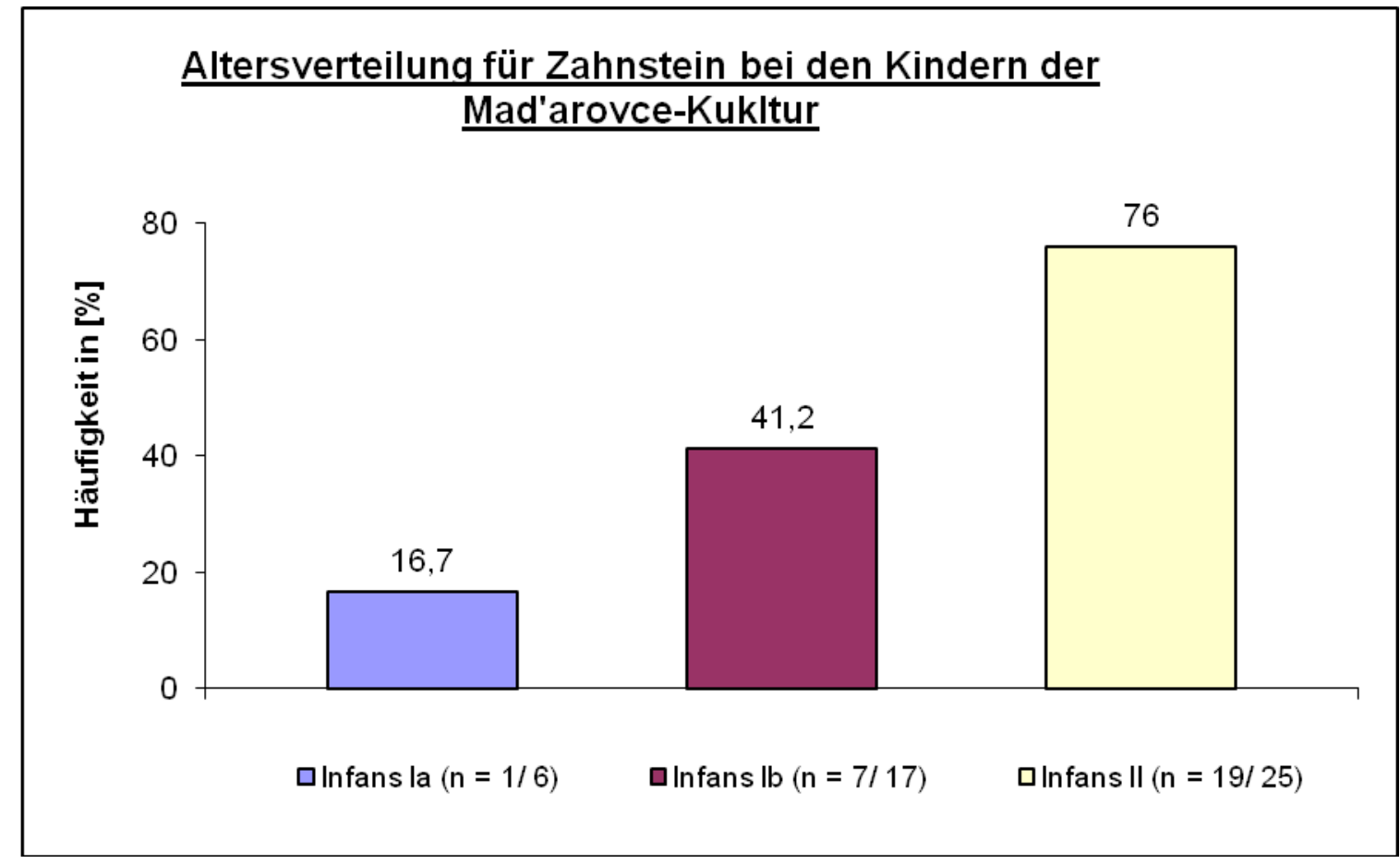

Abb. 13 Verteilung von Parodontopathien bei den Kindern der Mad'arovce-Kultur in Jelšovce in den einzelnen Altersklassen

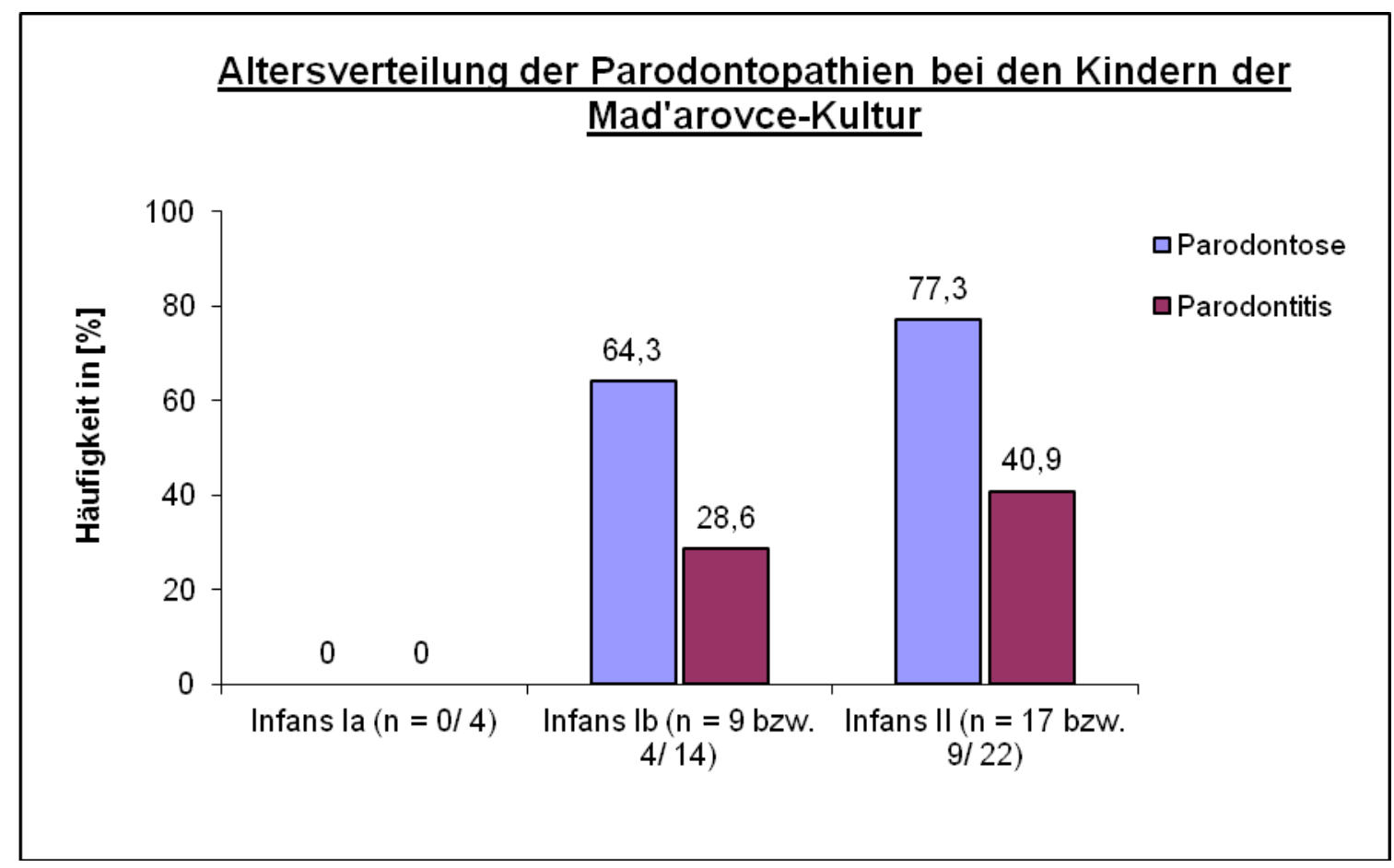

Alle Kinder mit einer Parodontitis leiden gleichzeitig auch an einer Parodontose. 
Abb. 14 Verteilung von transversalen Schmelzhypoplasien bei den Kindern der Mad'arovce-Kultur in Jelšovce in den einzelnen Altersklassen

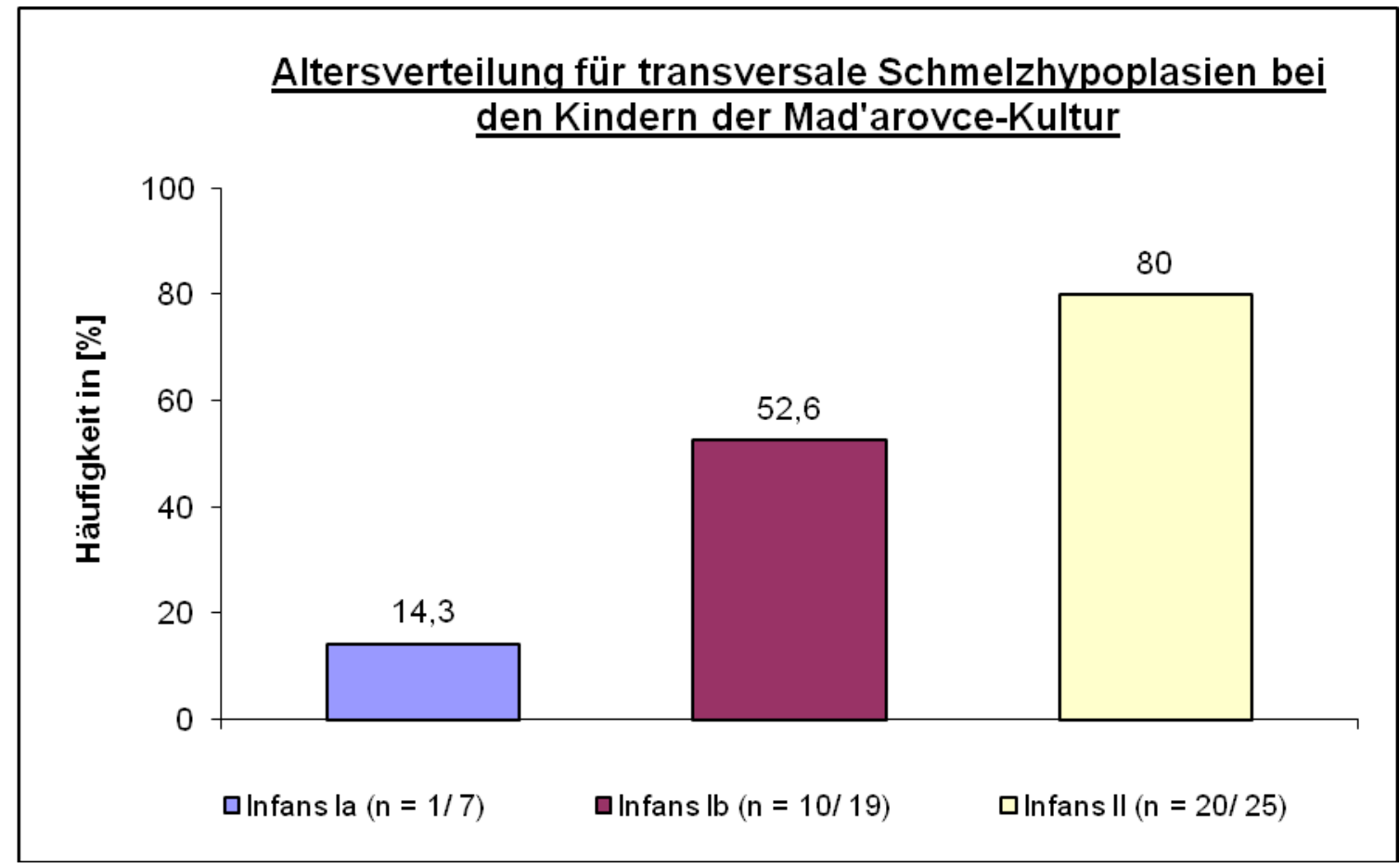

Abb. 15 Die Beziehung zwischen Zahnstein und Parodontopathien bei den Kindern der Mad'arovce-Kultur in Jelšovce.

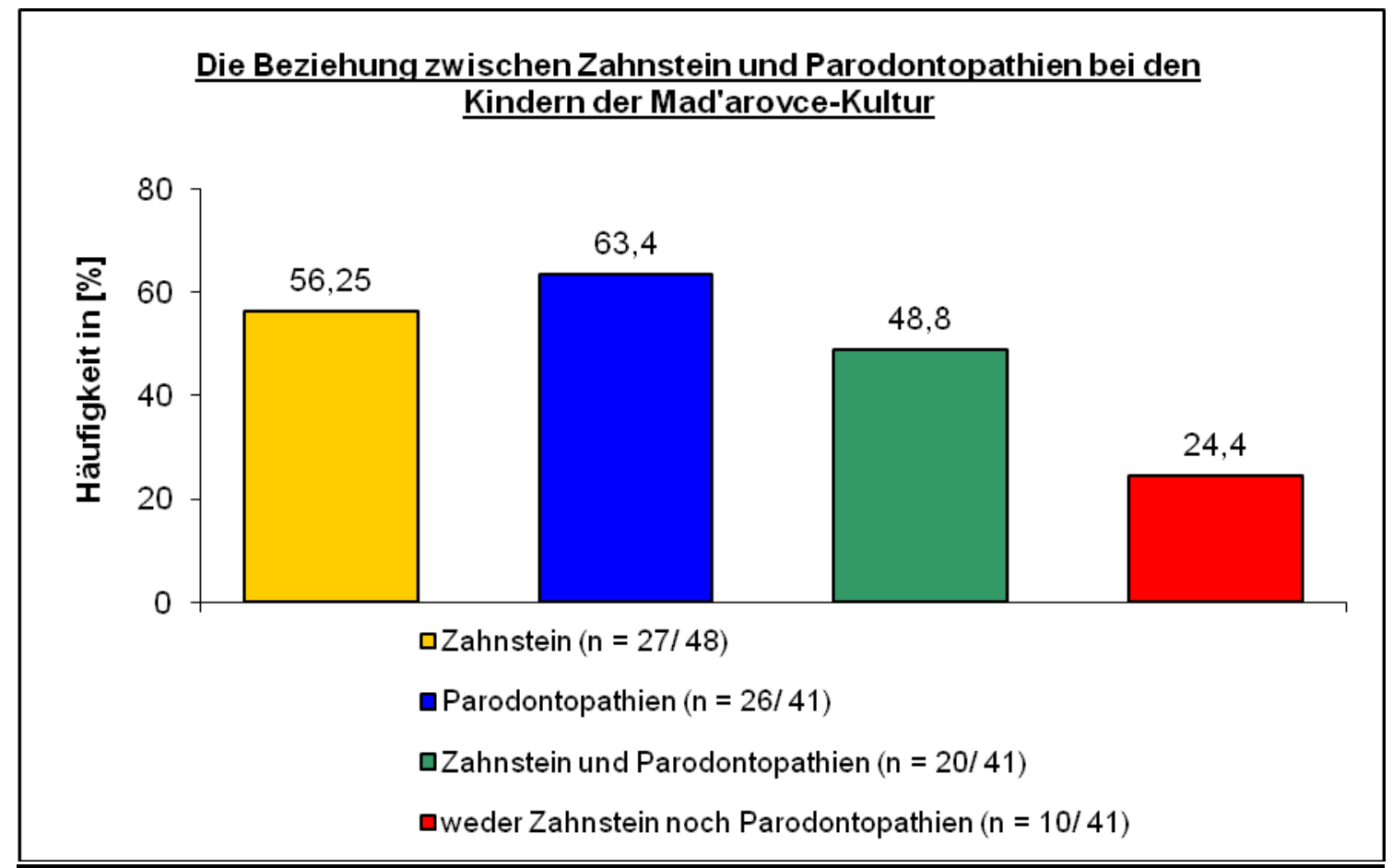




\section{III.4 Spuren pathologischer Prozesse am Postcranium}

\section{$\underline{\text { III.4.1 Veränderungen des Schultergürtels }}$}

\section{III.4.1.1 Clavicula}

Bei vier von 27 Kindern sind Enthesiopathien zu finden, bei zwei weiteren Kindern besteht der Verdacht darauf. Im Einzelnen zeigen sich grubige Vertiefungen, teilweise mit wulstigem Grund, bei drei Kindern im Ursprungsgebiet des Ligamentum coracoacromiale (Gräber 169, 220 und 566), bei einem Kind sternal im Ursprungsgebiet des Ligamentum costoclaviculare (Grab 158) und bei zwei Kindern acromial im Bereich des Ursprungs des M. deltoideus (Gräber 275 und 331).

Reste eines subperiostalen Hämatoms sind ventral und caudal im Bereich des Ursprungs des M. deltoideus bzw. des Lig. trapezoideum des Lig. coracoclavicularis bei einem Kind (Grab 65 A) nachweisbar.

Bei einem Kind (Grab 616 B) liegt eine beidseitige Dysplasie der sternalen Metaphysen mit einer Abflachung und Verringerung der S-Krümmung der Clavicula vor. Hinweise auf eine ausgeheilte Fraktur sind im Röntgenbild nicht zu finden (Tafel 18). Die Veränderungen sind wahrscheinlich genetisch bedingt.

Medizinischer Hintergrund:

Körperliche Überbeanspruchung kann im Bereich von Band-, Sehnen- und Muskelursprünge und -ansätze zu Nekrotisierung des angrenzenden Knochens führen. Diese Enthesiopathien treten bevorzugt im Ursprungsgebiet der Ligg. costoclaviculare und coracoclaviculare in Form von Gruben (Nekrosen) auf. Subperiostale Hämatome treten durch Abhebungen des Periosts ebenfalls in von Muskelzug beanspruchten Regionen auf.

Eine Abflachung und Verringerung der S-Krümmung der Clavicula sowie Verdrehungen des sternalen gegen das acromiale Ende können Hinweis auf eine ausgeheilte Fraktur, möglicherweise ein Geburtstrauma, sein. Im Röntgenbild sind häufig keine Frakturzeichen mehr auszumachen. 


\section{$\underline{\text { III.4.1.2 Scapula }}$}

Durch den Zug der an der Scapula entspringenden Muskeln (Mm. subscapularis, infra- und supraspinatus) manifestieren sich bei Skorbut besonders häufig Hämatome in den Fossae subscapularis, supra- und infraspinata. Reste eines subperiostalen Hämatoms zeigen sich bei zwei von 39 Kindern ( $=$ 2/ 39: 5,1 \%) (Gräber 126 und 83). Es sind jeweils einmal die Fossa subscapularis und die Fossa infraspinata betroffen.

\section{III.4.2 Veränderungen der oberen Extremitäten}

\section{$\underline{\text { III.4.2.1 Humerus }}$}

Spuren subperiostaler Hämatome lassen sich bei zwei von 49 Kindern (Gräber 65 A und 158) an der Diaphyse ventrolateral bzw. dorsal nachweisen ( $n=2 / 49: 4,1 \%)$ (s. auch Katalog). Bei dem Kind aus Grab 65 A ist die hämorrhagisch bedingte Auflagerung auch mikroskopisch nachweisbar (Tafel 21).

Weitere zwei Kinder (Gräber 72, 300 A) weisen feinporöse Areale im Bereich des Collums an der ventralen Seite auf. Der Grund von Fossa radialis und/ oder coronoidea sind bei fünf Kindern grobporös. Ob es sich dabei um genetisch bedingte Veränderungen, Wachstumszeichen oder Anzeichen einer Anämie handelt, ist unklar.

Eine Krempelung der proximalen Metaphysenplatten tritt bei einem Kind (Grab 158) auf (s. III.5.1).

Bei einem anderen Kind (Grab 346) sind die Fossae olecrani, radialis und coronoidea atypisch abgeflacht und im Vergleich zur Gegenseite nur sehr schwach ausgebildet. Die Unterarmknochen der betroffenen Seite sind nicht erhalten.

\section{III.4.2.2 Ulna und Radius}

Spuren subperiostaler Hämatome sind bei vier von 33 Kindern (n = 4/ 33: 12,1 \%) an einer Ulna und bei zwei von 39 Kindern $(n=2 / 39: 5,1 \%)$ an einem Radius erkennbar (Lokalisation s. Katalog Gräber 99, 158, 279 A, 346). Der bei dem Kind aus Grab 31 bestehende Ver- 
dacht einer Auflagerung am Radius konnte histologisch widerlegt werden. Die verdächtige Veränderung stellte sich als eine normale, im Wachstum befindliche Knochenstruktur heraus. Bei den Kindern aus den Gräbern 99, 158 und 279 A konnte die mikroskopische Untersuchung das Vorliegen hämorrhagisch bedingter Auflagerungen an den Ulnae bestätigen (Tafel 21 - 23). Die rechte Ulna des Kindes aus Grab 72 zeigt mikroskopisch eine Inaktivitätsatrophie.

Eine Krempelungen der distalen Metaphysen von Ulna und Radius kommen bei einem Kind (Grab 158 A) vor (s. auch Kapitel III.5.1.). Bei dem Kind aus Grab 275 ist der Ansatz des M. pronator teres grubenförmige vertieft.

\section{$\underline{\text { III.4.2.3 Hand }}$}

Das Kind aus Grab 65 A weist am Metacarpale II der rechten Hand eine subperiostale, hämorrhagisch bedingte Auflagerung in der Umgebung eines Foramen nutricium auf (Tafel 23).

\section{III.4.3 Veränderungen der Rippen}

An der Innenfläche eines Rippenfragmentes weist das Kind aus Grab 65 A Reste eines subperiostalen Hämatoms auf. Das Kind aus Grab 158 zeigt an der Innenfläche einer Rippe eine flache, muldenförmige Vertiefung. Es bestand der Verdacht auf einen druckatrophischen Prozess. Mikroskopisch und rasterelektronenmikroskopisch stellte sich die Läsion jedoch als postmortal verursacht heraus (Tafel 24). Spuren entzündlicher Prozesse auf den Rippeninnenflächen („Pleuritis“) sind nicht zu finden.

Aufgetriebene sternale Enden der Rippen lassen sich bei keinem Kind nachweisen. Bei zwei von 48 Kindern (Gräber 299 und 340) sind die Rippen insgesamt auffallend verdickt und verplumpt. Bei dem Kind aus Grab 299 konnten mikroskopisch keine erweiterten Markräume festgestellt werden, so dass sich der Verdacht auf eine Anämie nicht bestätigte. Auch die mikroskopische Untersuchung des Orbitadaches dieses Kindes gibt keinen eindeutigen Hinweis 
auf eine Anämie (vgl. III.2.6). Auch bei dem Kind aus Grab 340 gibt es keine weiteren Hinweise auf eine Anämie (vgl. III.2.6).

Medizinischer Hintergrund:

Sowohl hämorrhagische als auch entzündliche Prozesse können an den Rippeninnen- und -außenflächen ihre Spuren hinterlassen. Subperiostale Hämatome an den Innenflächen der Rippen treten bei Frakturen und Skorbut auf. Bei Rachitis und Skorbut können die sternalen Enden der Rippen (im Bereich der Knorpel-KnochenGrenzen) trichterförmig oder knotig erweitert sein. Diese Veränderungen entsprechen dem „rachitischen“ bzw. „skorbutischem Rosenkranz“. Sind nicht nur die Enden sondern die gesamte Rippe aufgetrieben und verplumpt und in ihrem Querschnitt fast quadratisch, kann dies der Hinweis auf eine Expansion des roten Knochenmarkes aufgrund einer Anämie sein.

\section{III.4.4 Veränderungen des Brustbeins}

Das Kind aus Grab 279 A (9 bis 10,5 Jahre) zeigt eine untypische Variante der Verknöcherung des Sternums auf. Manubrium und oberes Segment des Corpus sind vollständig synostosiert. Die übrigen Segmente des Corpus sind altersentsprechend noch nicht synostosiert. Es könnte sich um eine atypische Verlagerung der Synchondrosis sternalis zwischen erstem und zweitem Segment des Corpus sterni mit frühzeitigem Verschluss der Knorpelfuge zwischen Manubrium und erstem Corpussegment handeln (Tafel 24).

\section{III.4.5 Veränderungen der Wirbelsäule}

Das Kind aus Grab 350 zeigt eine leichte Variante des Klippel-Feil-Syndroms. Die Wirbelbögen der Halswirbel C5 und C6 dieses Kindes sind auf der rechten Seite fast vollständig ankylosiert. Die Wirbelkörper sind von der Ankylosierung nicht betroffen. Es besteht eine Spina bifida des 6. Halswirbels. Der Processus spinosus des ersten Brustwirbels ist gespalten (aberrante Form der Spaltbildung). Der Bogen ist leicht asymmetrisch, die linke Hälfte ist etwas nach cranial verzogen (Tafel 25). Diese angeborene partielle Blockwirbelbildung hat zu Lebzeiten des Kindes vermutlich zu keinerlei Beschwerden geführt. Bei einem weiteren Kind 
(Grab 126) besteht ebenfalls eine Spina bifida. Hier ist der 3. Sakralwirbel von der Spaltbildung betroffen (Tafel 26). Neben der Spina bifida weist dieses Kind auch eine Verdrehung der Processus spinosi der oberen Brustwirbel nach rechts auf. Die Foramina vertebralia sind asymmetrisch. Auch zwei weitere Kinder (Grab 228 B und 318) haben asymmetrisch verzogene Lendenwirbel allerdings ohne eine seitliche Verdrehung der Processus spinosi. Bei allen drei Kindern besteht der Verdacht auf eine genetisch bedingte Skoliose (Tafel 26).

Medizinischer Hintergrund:

An der Wirbelsäule sind vielfältige angeborene Fehlbildungen bekannt. Aplasien und Hypoplasien ganzer Wirbel oder Wirbelabschnitte sind möglich, ebenso Spaltbildungen an den verschiedensten Stellen sowie Ankylosierungen, d.h. Verschmelzungen ganzer Wirbel oder Teile davon (komplette / partielle Blockwirbelbildung). Häufig sind Kombinationen verschiedener Fehlbildungen. Bei einer Spina bifida spinalis handelt es sich um eine Hemmungsmissbildung, die in de Regel in den ersten drei Monaten der Schwangerschaft entsteht. Der mediale Schluss des Wirbelkanals kommt nicht zustande. Es können einzelne oder mehrere Segmente in Folge betroffen sein. Die häufigste Lokalisation für derartige Spaltbildungen sind die Übergangsregionen zwischen verschiedenen Wirbelsäulenabschnitten, besonders ist der lumbosacrale Übergang betroffen (BARNES 1994, BROCHER \& WILLERT 1980). Das Klippel-Feil-Syndrom ist eine wahrscheinlich genetisch bedingte Erkrankung, die mit Blockwirbelbildungen der Halswirbelsäule, am häufigsten im Bereich C2/3, gefolgt von C5/6, einhergeht (BARNES 1994, BROCHER \& WILLERT 1980). Es sind vor allem die Wirbelkörper, seltener auch die Wirbelbögen betroffen. Im betroffenen Bereich kommen zusätzlich gehäuft Spaltbildungen in Form einer Spina bifida vor. Skoliosen sind heute eine häufige Erscheinung bei heranwachsenden Kindern. Es handelt sich um fixierte seitliche Verkrümmungen der Wirbelsäule mit Verdrehung der einzelnen Wirbel (Wirbelkörper und Foramen vertebrale könne asymmetrisch sein) und sekundär der Rippen.

\section{III.4.6 Veränderungen des Beckens}

\section{$\underline{\text { III.4.6.1 Os ilii }}$}

Bei dem Kind aus Grab 65 A sind Spuren eines subperiostalen Hämatoms nachweisbar (n = 1/ 36: 2,8 \%) (s. Katalog, Tafel 23). Auf der Ala des Os ilii zwischen Y-Naht und Spina iliaca 
anterior superior weisen vier von 28 Kindern ein Areal mit mittel- bis grobporöser Oberfläche auf.

\section{$\underline{\text { III.4.6.2 Os ischii }}$}

Bei dem Kind aus Grab 299 ist die Fossa acetabuli beidseits über die Norm grobporös (Tafel 27). Ob es sich bei diesen Veränderungen um ein physiologisches Phänomen des Wachstums oder um einen krankhaften Prozess handelt ist noch unklar. Das Kind aus Grab 99 zeigt ventral zwischen Tuber ischiadicum und Y-Naht ein fein- bis mittelporöses Areal. Die Bedeutung ist ebenfalls noch unklar.

\section{III.4.7 Veränderungen der unteren Extremitäten}

\section{$\underline{\text { III.4.7.1 Femur }}$}

Makroskopisch zeigen sieben von 49 Kindern $(n=7 / 49: 14,3$ \%) Reste von plattenartigen Auflagerungen. Bei sechs der Kinder sind die Veränderungen an der Dorsalseite des Femur medial oder lateral der Linea aspera gelegen. Nur bei dem Kind aus Grab 254 B lässt sich die Auflagerung an der Ventralseite beobachten (s. Katalog Gräber 95, 134 B, 169, 220, 254 B, 346, 604 B). Die mikroskopische Untersuchung bestätigte bei den Kindern aus den Gräbern 220 und 604 B, dass es sich um hämorrhagisch bedingte Auflagerungen handelt (Tafel 29). Auch bei den Kindern aus den Gräbern 169 und 254 B ist eine hämorrhagische Ursache der Auflagerungen sehr wahrscheinlich (Tafeln 30 und 31). Bei dem Kind aus Grab 134 B ist mikroskopisch keine Auflagerung nachweisbar, stattdessen finden sich Zeichen eines appositionellen Wachstums mit rezidivierenden Wachstumsstillständen. Bei dem Kind aus Grab 95 konnten die beidseitigen Veränderungen mikroskopisch ebenfalls nicht auf hämorrhagisch bedingte Auflagerungen zurückgeführt werden. Es zeigen sich Spuren des normalen Wachstumsprozesses (Tafel 28).

Bei fünf von 22 Kindern lässt sich eine Krempelung der distalen Metaphysenplatte erkennen $(n=5 / 22: 22,7 \%)(v g l . ~ I I I .5 .1).(T a f e l ~ 50)$. 
Das Kind aus Grab 318 weist am Femur im Bereich des Trochanters minor eine exostotische Neubildung auf, die wahrscheinlich von einem Bruch in Bereich des Femurhalses herrührt (Tafel 34). Das Femur ist im Vergleich zur Gegenseite als Zeichen einer Inaktivität deutlich atrophisch. Auch das Röntgenbild zeigt den Dichteunterschied im Vergleich zur Gegenseite. Das Kind aus Grab 135 A weist beidseits an der Tuberositas gluteae reiskorngroße, knotige Exostosen auf (Tafel 34). Bei dem Kind aus Grab 566 sind der Ursprung des M. vastus medialis sowie der Ansatz des M. iliopsoas grubig vertieft. Diese Phänomene sind auf eine Überbeanspruchung des entspringenden bzw. ansetzenden Muskeln zurück zuführen.

Bei 15 von 40 Kindern ( $\mathrm{n}=$ 15/ 40: 37,5 \%) ist das Collum des Femurs fleckförmig grobporös (Tafel 39).

Bei dem Kind aus Grab 103 B ist die craniale Fläche des Collums stark netzartig porös. Mikroskopisch zeigt sich eine aufgelockerte poröse Oberfläche und radiär ausgerichtete Spongiosabälkchen sowie eine deutlich vermehrte Resorption im Bereich der Corticalis. Rasterelektronenmikroskopisch sind vermehrt Gefäßforamina bei starker postmortaler Verfremdung zu erkennen (Tafel 33). Differentialdiagnostisch handelt es sich entweder um überschießendes Wachstum oder um eine genetisch bedingte normale Wachstumsvariante. Zeichen für eine Osteomyelitis sind nicht zu finden. Eine Spongiosavermehrung bei Anämie wäre ebenfalls denkbar. Allerdings lassen sich an den übrigen Teilen des Skeletes keine weiteren Hinweise auf eine Anämie finden.

Das Kind aus Grab 340 weist beidseits eine Coxa valga auf (s. III.4.7.2).

Medizinischer Hintergrund:

Reste subperiostaler Hämatome stellen sich als fein- bis mittelporöse, relativ flache, der Knochenoberfläche aufgelagerte, knöcherne Neubildungen dar. In fortgeschrittenen Heilungsstadien wird die Oberfläche der Auflagerung zunehmend glatter. Die neugebildeten Strukturen werden in den alten Knochen integriert und sind später kaum noch als solche zu erkennen. Frakturen von Langknochen stellen sich je nach Ausheilungsstadium unterschiedlich dar. Die Kallusbildung ist mehr oder weniger ausgeprägt. Ausgeheilte Frakturen sind nur noch als 
Achsabweichungen und geringgradige Verdickungen des ehemaligen Frakturbereichs zu erkennen. Perimortal entstandene Brüche weisen keinerlei Heilungsspuren auf.

\section{$\underline{\text { III.4.7.2 Tibia und Fibula }}$}

Bei vier von 41 Kindern ( $=$ 4/ 41: 9,6 \%) sind makroskopisch an den Tibiae Spuren subperiostaler Hämatome nachweisbar. Sie sind bei zwei Kindern (Gräber 95 und 334) an der Facies posterior, bei einem Kind an der Facies medialis (Grab 220) und bei dem Kind aus Grab 254 B lateral der Tuberositas tibiae zu finden (s. auch Katalog). Mikroskopisch konnte die hämorrhagische Ursache der Auflagerungen für zwei der Kinder (Gräber 254 B und 334) belegt werden (Tafel 29 und 32). Das Kind aus Grab 95 weist mikroskopisch im Entstehen begriffene Haverssysteme als Zeichen des normalen Wachstumsprozesses auf (Tafel 28). Die betroffene Tibia aus Grab 220 wurde nicht mikroskopisch untersucht. Das Femur desselben Kindes zeigt aber eine mikroskopisch nachgewiesene, hämorrhagisch bedingte Auflagerung (s. III.4.7.1) (Tafel 29). Bei dem Kind aus Grab 134 B ist an der rechten Tibia eine längsspaltige Oberfläche zu finden. Rasterelektronenmikroskopisch stellt sich eine ausgeprägte, intravital poröse Oberfläche mit ovalen, spaltigen Foramina dar. Mikroskopisch sind ebenfalls intravital entstandene allerdings postmortal stark verfremdete Gefäßspalten zu erkennen. Am ehesten handelt es sich um den Rest einer postmortal erodierten, sehr gut integrierten, durchbauten Auflagerung (Tafel 35).

An den Fibulae sind bei vier von 34 Kindern Auflagerungen zu sehen (n = 4/ 34: 11,8 \%). Der Verdacht auf eine Auflagerung besteht bei vier weiteren Kindern (11,8 \%). Überwiegend sind die Auflagerungen medial, bei zwei Kindern auch lateral, des Margo anterior zu beobachten (s. auch Katalog Gräber 95, 113, 126, 169, 299, 350, 566 und 626) (Tafeln 30, 36 und 38). Mikroskopisch lassen sich bei zwei Kindern subperiostale Auflagerungen nachweisen (Gräber 299 und 626) (Tafel 36 - 38). Bei dem Kind aus Grab 95 bleibt der Verdacht bestehen, eine Auflagerung ist jedoch nicht sicher nachweisbar. 
Eine Krempelungen der proximalen Metaphysenplatten der Tibia tritt bei einem Kind (Grab 88 A) auf.

Bei elf von 33 Kindern ( $=11$ / 33: 33,3 \%) sind an den Tibiae grubige, spindelförmige Vertiefungen der Linea musculi solei zu beobachten. Es handelt sich hierbei um Enthesiopathien, die ähnlich wie bei den Claviculae mit einer Überbeanspruchung der Muskeln (M. soleus) in Zusammenhang stehen (Tafel 34).

Harris-Linien lassen sich röntgenologisch bei 17 von 19 zur Untersuchung geeigneten Individuen feststellen ( $\mathrm{n}=17 /$ 19: 89,5 \%). Insgesamt wurden 31 Tibiae untersucht. Die Anzahl der Harris-Linien variiert von einer bis zu 22 Stück pro Metaphyse, durchschnittlich sind zehn bis zwölf Linien nachweisbar (Tafel 39).

Bei dem Kind aus Grab 616 B fällt die linke Tibia durch eine massive Verdickung des gesamten Knochens auf (Tafel 40); besonders die mediale Oberfläche ist deutlich vorgewölbt. Die proximale Epiphyse weist einen atypischen Verknöcherungssporn, der in die zentral aufgelockerte Diaphyse reicht (Tafel 40). Endoskopisch zeigt sich in der Diaphyse die Spongiosa atypisch dünn und fadenartig verändert (Tafel 41). Histologisch ist der Markraum erweitert; dort wo die Vorwölbung besteht, ist die Compacta ausgedünnt. Die atypisch konfigurierte Spongiosa an einer Stelle der distalen Diaphyse, an der eigentlich keine Spongiosa sein sollte, ist ebenfalls zu erkennen (Tafeln 41). Zeichen eines aggressiven Knochenumbaus wie bei Osteomyelitis sind nicht zu finden. Die rechte Seite ist unauffällig. Es besteht der Verdacht auf einen ossären Tumor (s. IV.8.3).

Bei dem Kind aus Grab 300 A sind Tibia und Fibula der rechten Seite deutlich verplumpt. Die Kanten sind abgerundet. Beide Knochen weisen eine Verbiegung nach medial auf (Tafel 42). Im Röntgenbild ist eine deutliche Verbreiterung der Compacta besonders im Schaftbereich zu erkennen (Tafel 47). Mikroskopisch zeigt sich eine der ehemaligen Oberfläche aufgelagerte Knochenneubildung im Sinne einer Periostose (Tafel 43 und 44). Die rechte Tibia ist dabei stärker als die rechte Fibula befallen, etwa zwei Drittel der gesamten Oberfläche sind betrof- 
fen. Im mikroskopischen Bild sind die Kollagenfasern im Bereich der Oberfläche senkrecht zur Oberfläche orientiert (Tafel 43). Es handelt sich um gut differenzierten Faserknochen, der einen beginnenden Umbau zu Lamellenknochen aufweist (Tafel 44). Zeichen einer Osteomyelitis sind nicht zu finden. Auch die makroskopisch gesunde Seite weist in schwächerer Ausprägung dieselben periostotischen Veränderungen auf. Es handelt sich um einen langsam fortschreitenden Prozess, der in unterschiedlicher Ausprägung die Unterschenkelknochen des Kindes befallen hat. Was ursächlich für diese Veränderungen ist, konnte bisher nicht geklärt werden. Von den heute bekannten Knochendysplasien kommt lediglich die Chondrodysplasia punctata in Frage. Bei dieser Erkrankung kann es zu ausgeprägten Unterschieden im Ausmaß des Befalls der verschiedenen Knochen kommen. Häufig tritt ein einseitiger Befall einer Extremität auf (SPRANGER et al. 1974). Typisch sind vor allem die Veränderungen im Bereich der Epiphysen. Es wären auch noch weitere Knochendeformitäten zum Beispiel an Wirbelkörpern und Gesichtsknochen zu erwarten (SPRANGER et al. 1974), welche bei diesem Kind an den vorhandenen Knochen jedoch nicht zu finden sind. Daher ist es sehr fraglich, ob das Kind wirklich an einer Chondrodysplasie litt.

Bei dem Kind aus Grab 15 sind alle Knochen der unteren Extremitäten über die Norm grazil (Tafel 45). Femura und Humeri haben fast den gleichen Durchmesser. Im Röntgenbild erscheint die Compacta ausgedünnt (Tafel 47). Die Knochen der oberen Extremitäten sind normal bis kräftig entwickelt. Die Femura zeigen histologisch eine Reduktion der Compacta, die von der Markhöhle ausgeht (Tafeln 46). Es findet sich eine vermehrte osteoklastische Resorption im Bereich der Compacta (Tafeln 44 und 46). Rechte und linke Seite sind bei diesem Kind gleich stark betroffen. Knochenneubildungen im Bereich der Linea aspera schließen aber eine ausgeprägte Inaktivitätsatrophie aus (Tafel 46). Typische Zeichen einer Osteomyelitis sind ebenfalls nicht zu finden. Offensichtlich handelt es sich um eine chronische, systemische Erkrankung, die in dieser Form heute nicht mehr auftritt. 
Das Kind aus Grab 340 weist neben einer Coxa valga beidseits (s. III.4.7.1) sehr grazile Knochen der unteren Extremitäten auf. Im Röntgenbild ist eine Doppelkontur der Corticalis zu erkennen. Auch mikroskopisch ist diese Doppellamelle nachweisbar. Zwischen innerer und äußerer Generallamelle befindet sich kaum kompakter Knochen (Tafel 48). Die Spongiosa ist reduziert, die Corticalis stark ausgedünnt. Im Faserknochen der Corticalis befinden sich erste Primärosteone. Aufgrund der offensichtlich sehr wenig mechanisch beanspruchten Knochen (Inaktivitätsatrophie) liegt der Verdacht nahe, dass dieses Kind trotz seiner ein bis zwei Jahre noch nicht gelaufen ist. Vermutlich war es zu krank um Laufen zu lernen oder ist längere Zeit bettlägerig gewesen.

Medizinischer Hintergrund:

Zahlreiche Knochentumoren manifestieren sich bevorzugt in der Knieregion. Sie können zu einer lokalisierten Verdickung und Verplumpung des Knochens führen. Harris-Linien entstehen bei schweren Erkrankungen oder durch Mangelernährung. Das Wachstum wird zugunsten der Gesundung eingestellt. Sie werden nach Wachstumsstillständen bei Wiedereinsetzen des Wachstums als verdichtete Linien in den Metaphysenbereichen der Langknochen (bevorzugt erkennbar an den Tibiae) im Röntgenbild sichtbar. Bei chronisch unterernährten Kindern lassen sich Harris-Linien aufgrund des dauerhaft langsamen Wachstums nur in geringer Ausprägung oder gar nicht nachweisen (MC HENRY \& SCHULZ 1976). Harris-Linien können im Laufe des Lebens durch Umbauvorgänge des Knochens resorbiert werden und damit verschwinden.

\section{$\underline{\text { III.4.7.3 Fuß }}$}

Das Os metatarsale I der rechten Seite des Kindes aus Grab 99 zeigt im distalen Ende eine atypische, fadenförmige Spongiosa. Wahrscheinlich handelt es sich um eine ausgeheilte Mikrofraktur, alternativ auch um eine Metastase oder einen ossären Tumor. Allerdings gibt es bei diesem Kind keine weiteren Hinweise auf eine tumoröse Neubildung. 


\section{III.5 Mangelerkrankungen}

In Jelšovce sind zwei Drittel der Kinder der Mad’arovce-Kultur von Mangelerkrankungen betroffen ( $\mathrm{n}=39$ / 59: 66,1 \%).

$\underline{\text { III.5.1 infantiler Skorbut = Möller-Barlowsche-Erkrankung }}$

Bei zwölf von 49 Kindern ( $=12$ / 49: 24,5 \%) kann ein Skorbut nachgewiesen werden. Bei drei weiteren Kindern (Gräber 126, 279 A und 337) besteht diesbezüglich ein Verdacht.

Ein Skorbut lässt sich nicht anhand einzelner Veränderungen diagnostizieren. Erst die Kombination verschiedener Merkmale lässt eine Diagnose zu. Typisch sind neben erhöhter Infektanfälligkeit und bestimmten Wachstumsstörungen vor allem subperiostal gelegene Blutungen. Diese hinterlassen an den Knochen sogenannte hämorrhagisch bedingte Auflagerungen, die sich mikroskopisch nachweisen lassen. Dieser neugebildete Knochen ist der originalen Lamina externa oder Corticalis aufgelagert, die nicht strukturell verändert ist (z. B. Tafeln 21 - 23, 29, 36 und 38). Auch die darunter liegende Spongiosa ist normal strukturiert. Bei gut integrierten, älteren Auflagerungen ist die ehemalige Lamina externa bzw. interna; äußere Compacta bzw. Corticalis häufig nur noch als zur Oberfläche parallel verlaufender Streifen erkennbar. Ob diese Auflagerungen traumatisch, im Rahmen eines Skorbut oder einer Gerinnungsstörung entstanden sind, ist nicht zu erkennen. Lediglich der hämorrhagische Ursprung der Veränderung ist nachweisbar. Von Rachitis und Anämie lässt sie sich eindeutig unterscheiden.

Die folgende Tabelle (Tab. 7 a und b) listet Veränderungen auf, die im Rahmen eines Skorbuts auftreten können. 
Tab. 7 a und b Merkmale eines Skorbuts am mazerierten Knochen

(ASCHOFF 1919, BARLOW 1883, 1895 a und 1895 b, BRICKLEY \& IVES 2006, FRAENKEL 1908, HART \& Lessing 1913, JAfFe 1972, Klose 1919, MÖller 1862, ORTNer \& ERICKSEN 1997, ORTNER et al. 2001, ORTNER 2003, SCHULTZ 2001 a, 2001 b und 2003)

Tab. 7 a Veränderungen am Cranium

\begin{tabular}{|c|c|c|}
\hline $\begin{array}{l}\text { betroffene } \\
\text { Strukturen }\end{array}$ & Lokalisation der Veränderungen & Art der Veränderungen \\
\hline L. interna & in den Abdrücken der Hirnwindungen & $\begin{array}{l}\text { hämorrhagisch bedingte } \\
\text { Auflagerungen und atypische } \\
\text { Gefäßimpressionen }\end{array}$ \\
\hline L. externa & $\begin{array}{l}\text { besonders im Bereich der } \\
\text { Wachstumshöcker }\end{array}$ & $\begin{array}{l}\text { hämorrhagisch bedingte } \\
\text { Auflagerungen }\end{array}$ \\
\hline Unterkiefer & $\begin{array}{l}\text { Innenfläche des Ramus mandibulae } \\
\text { im Bereich des Zahnhalteapparates und der } \\
\text { Alveolarränder } \\
\text { porös ausgekleidete Alveolen }\end{array}$ & $\begin{array}{l}\text { hämorrhagisch bedingte } \\
\text { Auflagerungen und verstärkte } \\
\text { Porosität }\end{array}$ \\
\hline Oberkiefer & $\begin{array}{l}\begin{array}{l}\text { im Bereich des Zahnhalteapparates und der } \\
\text { Alveolarränder }\end{array} \\
\text { porös ausgekleidete Alveolen } \\
\text { im Bereich des Foramens infraorbitale } \\
\text { dorsale Fläche der Maxilla }\end{array}$ & $\begin{array}{l}\text { hämorrhagisch bedingte } \\
\text { Auflagerungen und verstärkte } \\
\text { Porosität }\end{array}$ \\
\hline Orbita & Orbitadach und laterale Wand & $\begin{array}{l}\text { hämorrhagisch bedingte } \\
\text { Auflagerungen, poröse } \\
\text { Oberfläche }\end{array}$ \\
\hline Jochbogen & ventrale und dorsale Fläche & $\begin{array}{l}\text { hämorrhagisch bedingte } \\
\text { Auflagerungen }\end{array}$ \\
\hline $\begin{array}{l}\text { harter } \\
\text { Gaumen }\end{array}$ & vor allem im hinteren Anteil, Stomatitis & $\begin{array}{l}\text { verstärkte Porosität und / oder } \\
\text { poröse Auflagerungen }\end{array}$ \\
\hline $\begin{array}{l}\text { Os } \\
\text { sphenoidale }\end{array}$ & $\begin{array}{l}\text { Außenseite der Ala major } \\
\text { Auflagerungen auf der Ala minor }\end{array}$ & $\begin{array}{l}\text { verstärkte Porosität und / oder } \\
\text { poröse Auflagerungen } \\
\text { hämorrhagisch bedingte } \\
\text { Auflagerungen }\end{array}$ \\
\hline Os temporale & Außenseite der Squama temporalis & $\begin{array}{l}\text { verstärkte Porosität und/ oder } \\
\text { hämorrhagisch bedingte } \\
\text { Auflagerungen }\end{array}$ \\
\hline
\end{tabular}


Tab. 7 b Veränderungen am Postcranium

\begin{tabular}{|l|l|l|}
\hline $\begin{array}{l}\text { betroffene } \\
\text { Strukturen }\end{array}$ & Lokalisation der Veränderungen & Art der Veränderungen \\
\hline Langknochen & $\begin{array}{l}\text { Epi- und Diaphysen, bevorzugt Femur und } \\
\text { Tibia }\end{array}$ & $\begin{array}{l}\text { hämorrhagisch bedingte } \\
\text { Auflagerungen }\end{array}$ \\
\cline { 2 - 3 } & Metaphysen & $\begin{array}{l}\text { Osteophytenbildung ("gekrempel- } \\
\text { te Metaphysenplatten") }\end{array}$ \\
\hline Schulterblatt & $\begin{array}{l}\text { Fossa subscapularis, supra- und } \\
\text { infraspinata }\end{array}$ & $\begin{array}{l}\text { hämorrhagisch bedingte } \\
\text { Auflagerungen }\end{array}$ \\
\hline Becken & bevorzugt Fossa iliaca & $\begin{array}{l}\text { hämorrhagisch bedingte } \\
\text { Auflagerungen }\end{array}$ \\
\hline Rippen & im Bereich der Knorpel-Knochengrenzen & $\begin{array}{l}\text { hämorrhagisch bedingte } \\
\text { Auflagerungen }\end{array}$ \\
\hline
\end{tabular}

Im Einzelnen sind bei den skorbutkranken Kindern der Mad'arovce-Kultur die folgenden Veränderungen nachweisbar: Auflagerungen auf der Lamina externa zeigt ein Kind (Grab 72) (n = 1/ 13) (s. III.2.2). Bei einem anderen Kind (Grab 88 A) sind im Rahmen des Skorbuts hämorrhagisch bedingte, plattenartige poröse Auflagerungen auf der Lamina interna aufgetreten (Tafel 49). Bei fünf weiteren Kindern sind atypische Gefäßimpressionen zu sehen (n = 5/ 11) (s. III.2.3) (Tafeln 6, 7 und 49). Auflagerungen an der Innenseite des Ramus mandibulae zeigt nur das Kind aus Grab 65 A ( $n=1 / 9)$. Bei dem Kind aus Grab 95 ist die Innenfläche des Ramus mandibulae verstärkt porös $(n=1 / 9)$. Durch den Zahndurchbruch können die Alveolar-ränder porös verändert sein, bei Skorbut kommt es zu einer massiven Verstärkung der Porosität kombiniert mit dem Auftreten poröser Auflagerungen. Auflagerungen im Bereich des Zahnhalteapparates und der Alveolarränder zeigen elf von 13 an Skorbut erkrankter Kinder (s. III.3.7) (Tafel 50). Porös ausgekleidete Alveolen lassen sich bei fünf Kindern beobachten, von diesen ist aber nur bei drei Kindern ein Skorbut sicher zu diagnostizieren $(n=3 / 13)$. Die Umgebung des Foramens infraorbitale ist bei vier von sieben betroffenen Kindern, die Dorsalfläche der Maxilla ist bei fünf von sieben an Skorbut leidenden Kindern über die Norm porös (Tafel 49). Von neun skorbutkranken Kindern mit erhaltenen Orbitadächern leiden acht an einer Cribra orbitalia (Tafeln 12 - 14). Der Jochbogen ist bei zwei von zehn Kindern (Gräber 6 B 
und 336 B) über die Norm porös. Eine Stomatitis ist bei sechs von zehn Kindern zu beob-achten (s. III.2.10) (Tafel 17). Die Außenfläche der Ala major des Os sphenoidale sowie das angrenzende Os temporale zeigen bei drei von acht Kindern eine vermehrte Porosität (Tafel 50). Bei einem dieser Kinder (Grab 72) liegt eine Auflagerung vor. Die Ala minor weist bei drei Kindern eine Auflagerung auf (Gräber 6 B, 88 A und 301) (Tafel 50). An den Langknochen weisen 17 Kinder Auflagerungen auf. Bei acht Kindern stehen diese offenbar im Zusammenhang mit der Vitamin-C-Mangelerkrankung $(n=8 / 13)$. Bei porösen Oberflächen bzw. Auflagerungen an den Diaphysen der langen Röhrenknochen muss differentialdiagnostisch auch an die Entstehung durch chronische Herz-Lungen-Kreislauferkrankungen (z.B. PierreMarie-Bamberger-Syndrom, Cottey’s Disease) gedacht werden. Diese Veränderungen stellen sich allerdings mikroskopisch nicht als hämorrhagisch bedingt dar. Am Schulterblatt lassen sich bei zwei Kindern Auflagerungen nachweisen $(n=2 / 10)$ (s. III.4.1.2.). Das Becken ist nur bei dem Kind aus Grab 65 A betroffen (n = 1/8) (s. III.4.6.1) (Tafel 23). Dasselbe Kind weist im Rahmen des Skorbuts auch Auflagerungen auf einem Rippenfragment, einer Clavicula und dem Metacarpale II der rechten Hand auf (s. III.4.1.1, III.4.3 und III.4.2.3.) (Tafel 23). Die Metaphysenplatten der langen Röhrenknochen zeigen als Zeichen der Wachstumsstörung bei Skorbut bei sechs von 13 Kindern eine Osteophytenbildung und erscheinen daher nach hinten "gekrempelt". Betroffen sind bei vier Kindern das distale Femur, bei einem Kind (Grab 88 A) die proximale Tibia und bei einem weiteren Kind (Grab 158 A) distaler Radius, distale Ulna und proximaler Humerus (Tafel 50). Die Verteilung der Skorbutmerkmale bei den skorbutkranken Kindern der Mad'arovce-Kultur zeigen die Abbildungen 16 und 17. Cribra orbitalia zeigen 88,9 \% (n=8/9) der skorbutkranken Kinder. Nachweisbar hämorrhagischen Ursprungs sind sie jedoch nur bei 2 Kindern. Poröse Auflagerungen an den Alveolarknochen von Ober- und Unterkiefer treten mit fast 85\% (n = 11/ 13: 84,6 \%) ebenfalls häufig auf. Eine poröse Oberfläche der Dorsalfläche der Maxilla ist bei 71,4 \% der Kinder zu beobachten (n = 5/ 7) (Abb. 16 und 17). Ebenfalls häufig treten Auflagerungen auf den Langknochen sowie 
um das Foramen infraorbitale herum auf ( $n=8 / 13$ : 61,5 bzw. $n=4 / 7: 57,1 \%)$. Selten hingegen sind poröse Auflagerungen auf der Lamina externa des Schädeldaches ( $\mathrm{n}=1 /$ 13: 7,7 \%). Typischerweise treten diese im Bereich der Wachstumshöcker auf.

\section{$\underline{\text { III.5.1.1 Merkmale eines Skorbuts }}$}

In diesem Abschnitt wird das System vorgestellt, das Grundlage der Diagnosestellung eines Skorbuts in der vorliegenden Arbeit war. Die verschiedenen Veränderungen, die bei einem Skorbut auftreten können, werden in Merkmale I. und II. Ordnung eingeteilt, je nachdem wie typisch sie für das Auftreten eines Skorbutes sind. Merkmale I. Ordnung sind Auflagerungen auf Lamina externa und interna sofern sie hämorrhagisch bedingt sind. Unspezifische Veränderungen, z.B. atypische Gefäßimpressionen der Lamina interna werden lediglich als Merkmale II. Ordnung gewertet. Weitere Merkmale I. Ordnung sind porös ausgekleidete Alveolen und hämorrhagisch bedingte Auflagerungen an den Alveolarrändern von Ober- und Unterkiefer, da diese Veränderungen bei den für den Skorbut typischen Einblutungen in den Zahnhalteapparat entstehen. Ebenfalls Merkmale I. Ordnung sind hämorrhagisch bedingte Auflagerungen an Femura, Tibiae und Fibulae, da diese besonders häufig von subperiostalen Blutungen betroffen sind, sowie Osteophytenbildungen im Bereich der Metaphysen der langen Röhrenknochen im Sinne einer „Krempelung “ der Metaphysenplatte.

\section{Merkmale I. Ordnung:}

- hämorrhagisch bedingte Auflagerungen auf Lamina externa und interna des Schädeldaches und an Alveolarrändern (ASCHOFF 1919, BARLOW 1883 und 1895 a, BRICKLEY \& IVES 2006, HART \& LESSING 1913, ORTNER 2003, ORTNER et al. 2001, SCHULTZ 2001 a, $2001 \mathrm{~b}$ und 2003)

- porös ausgekleidete Zahnalveolen (ASCHOFF 1919, BARLOW 1883 und 1895 a, BRICKLEY \& IVES 2006, ORTNER 2003, SCHULTZ 2001 a)

- histologisch nachgewiesene hämorrhagisch bedingte Cribra orbitalia (ASCHOFF 1919, BARlOW 1895 a, BRICKLEY \& IVES 2006, CARLi-ThIELE \& SCHUlTZ 2001, HART \& 
LESSING 1913, JAFFE 1972, ORTNER 2003, SCHULTZ 2001 b und 2003)

- hämorrhagisch bedingte Auflagerungen an Femura, Tibiae, Fibulae und proximalen Ulnae (BARlow 1895 a, CARLi-Thiele \& SCHUltz 2001, FraenKel 1908, HART \& LESSING 1913, KLOSE 1919, ORTNER 2003, SCHULTZ 2001 a und 2001 b)

- Osteophytenbildungen im Bereich der Metaphysen der langen Röhrenknochen ("gekrempelte" Metaphysen) (FRAENKEL 1908, HART \& LESSING 1913, JAFFE 1972, ORTNER 2003, SCHUSTER 1990)

\section{Merkmale II. Ordnung:}

Diese sind weniger typische Veränderungen, die aber häufig im Rahmen eines Skorbuts auftreten.

- unspezifische Veränderungen an Lamina interna und externa des Schädeldaches

- Auflagerungen an der Innenseite des Ramus mandibulae und im Bereich des Foramen infraorbitale (BRICKLEY \& IVES 2006, HART \& LESSING 1913, ORTNER 2003)

- über die Norm verstärkte Porosität der Hinterfläche der Maxilla und des Processus zygomaticus (BARLOW 1883 und 1895 a, BRICKLEY \& IVES 2006, ORTNER 2003)

- verstärkte Porosität oder Auflagerung auf der Außenfläche der Ala major ossis sphenoidalis und der Squama temporalis (BRICKLEY \& IVES 2006, ORTNER \& ERICKSEN 1997, ORTNER 2003)

- hämorrhagisch bedingte Auflagerungen an nicht genannten Stellen des knöchernen Schädels (BRICKLEY \& IVES 2006)

- unspezifische Cribra orbitalia, ohne histologische Untersuchung

- Stomatitis (ASCHOFF 1919, BARLOW 1895 b, BRICKLEY \& IVES 2006, CARLI-THIELE \& SCHULTZ 2001, ORTNER 2003, SCHULTZ 2001 a)

- hämorrhagisch bedingte Auflagerungen an Humeri, Ulnae, Radii, Scapulae, Rippen, Beckenknochen oder anderen Knochen des Postcraniums (BRICKLEY \& IVES 2006, CARLITHIELE \& SCHULTZ 2001, ORTNER 2003) 
Tab. 8 Merkmale eines Skorbuts nach ihrer Wertigkeit

Tab. 8 a am Cranium

\begin{tabular}{|c|c|c|c|}
\hline & Cranium & I. Ordnung & II. Ordnung \\
\hline Auflagerun & en Lamina interna & hämorrhagisch & unspezifisch \\
\hline Auflagerun & en Lamina externa & hämorrhagisch & unspezifisch \\
\hline Mandibula & Aufl. Ramus mandibulae innen & XXXXXXxxxxxxxxx & hämorrhagisch \\
\hline & Alveolen innen poröse & vermehrt porös & XXXXXXXXXXXXXXX \\
\hline & Auflagerungen Alveolarrand & hämorrhagisch & 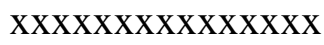 \\
\hline Maxilla & Auflagerungen Alveolarrand & hämorrhagisch & XXXXXXXXXXXXXXX \\
\hline & Alveolen innen porös & vermehrt porös & 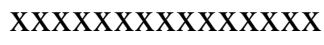 \\
\hline & Aufl. bei Foramen infraorbitale & 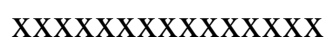 & hämorrhagisch \\
\hline & Dorsalseite der Kieferhöhle & XXXXXXXXXXXXXXX & vermehrt porös \\
\hline Cribra orbi & & $\begin{array}{l}\text { mit Histologie: } \\
\text { hämorrhagisch }\end{array}$ & $\begin{array}{l}\text { ohne Histologie, } \\
\text { unspezifisch }\end{array}$ \\
\hline Processus z & gomaticus, Dorsalseite & XXXXXXXXXXXXXXX & vermehrt porös \\
\hline Stomatitis & & XXXXXXXXXXXXXXX & vermehrt porös \\
\hline $\begin{array}{l}\text { Ala major c } \\
\text { angrenzend }\end{array}$ & $\begin{array}{l}\text { sis sphenoidalis, Außenseite und } \\
\text { s Os temporale }\end{array}$ & 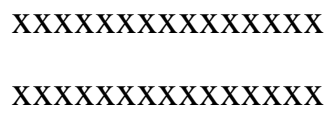 & vermehrt porös \\
\hline Auflagerun & en an nicht genannten Stellen & XXXXXXXXXXXXXXX & hämorrhagisch \\
\hline
\end{tabular}

Tab. 8 b am Postcranium

\begin{tabular}{|c|c|c|c|c|}
\hline \multicolumn{3}{|l|}{ Postcranium } & I. Ordnung & II. Ordnung \\
\hline \multirow{3}{*}{$\begin{array}{l}\text { Auflagerungen } \\
\text { untere } \\
\text { Extremitäten }\end{array}$} & \multicolumn{2}{|c|}{ Femur } & hämorrhagisch & XXXXXXXXXXXXxxx \\
\hline & \multicolumn{2}{|c|}{ Tibia } & hämorrhagisch & 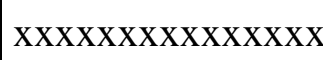 \\
\hline & \multicolumn{2}{|c|}{ Fibula } & hämorrhagisch & 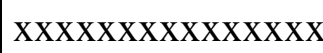 \\
\hline \multirow{4}{*}{$\begin{array}{l}\text { Auflagerungen } \\
\text { obere } \\
\text { Extremitäten }\end{array}$} & \multicolumn{2}{|c|}{ Humerus } & Xxxxxxxxxxxxxxx & hämorrhagisch \\
\hline & \multirow[t]{2}{*}{ Ulna } & proximal, Olecranon & hämorrhagisch & XXXXXXXXXXXXXXX \\
\hline & & distal, Mitte & 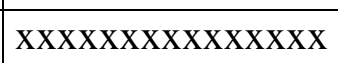 & hämorrhagisch \\
\hline & \multicolumn{2}{|c|}{ Radius } & Xxxxxxxxxxxxxxx & hämorrhagisch \\
\hline \multicolumn{3}{|c|}{ Auflagerungen Scapula } & Xxxxxxxxxxxxxxx & hämorrhagisch \\
\hline \multicolumn{3}{|c|}{ Auflagerungen Becken } & $\operatorname{xxxxxxxxxxxxxxx}$ & hämorrhagisch \\
\hline \multicolumn{3}{|c|}{ Auflagerungen Rippen } & 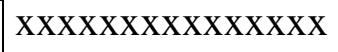 & hämorrhagisch \\
\hline \multicolumn{3}{|c|}{ Auflagerungen auf anderen Knochen } & 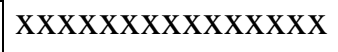 & hämorrhagisch \\
\hline \multicolumn{3}{|c|}{ "gekrempelte" Metaphysen } & Osteophyten & 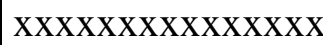 \\
\hline
\end{tabular}


Die Kombination der verschiedenen Merkmale lässt die Diagnose eines Skorbuts sicher bis wahrscheinlich sein. Treten in einer Kategorie bestimmte Merkmale mehrfach auf, z.B. Auflagerungen auf Femur rechts und links, so wird für dieses Merkmal jedoch trotzdem nur ein Punkt vergeben. Anders verhält es sich bei "Auflagerungen an nicht genannten Stellen" bzw. "auf anderen Knochen", hier wird für jeden betroffenen Knochen ein Punkt vergeben. Nach Berücksichtigung aller Merkmale wird die erzielte Punktzahl ausgewertet. Die folgende Tabelle (Tab. 9) zeigt, bei welchen Kombinationen ein Skorbut diagnostiziert wurde und bei welchen lediglich der Verdacht auf einen Vitamin-C-Mangel geäußert wurde.

Tab. 9 Verteilung der Merkmale zur Diagnostik eines Skorbuts

\begin{tabular}{|l|l|}
\hline Skorbut diagnostiziert & Verdacht auf Skorbut \\
\hline Merkmale I. $\geq 4 \mathrm{x}$ & Merkmale I. 0 x, II. $5 \mathrm{x}$ \\
\hline Merkmale I. $3 \mathrm{x}, \mathrm{II} . \geq 1 \mathrm{x}$ & Merkmale I. $1 \mathrm{x}$, II. $4 \mathrm{x}$ \\
\hline Merkmale I. 2 x, II. $\geq 3 \mathrm{x}$ & Merkmale I. 2 x, II. $2 \mathrm{x}$ \\
\hline Merkmale I. 1 x, II. $\geq 5 \mathrm{x}$ & Merkmale I. 3 x, II. 0 x \\
\hline Merkmale I. 0 x, II. $\geq 6 \mathrm{x}$ & \\
\hline
\end{tabular}

Zur Verdeutlichung werden einige Beispiele angeführt.
1). Grab 65 A:
(s. auch IV.8.1)

hämorrhagisch bedingte Auflagerungen an Alveolarrändern Maxilla (I.) und Mandibula (I.), atypische Gefäßimpressionen Lamina interna des Schädeldaches (II.), Dorsalseite Maxilla porös (II.), Cribra orbitalia (ohne Histologie) (II.), Stomatitis (II.), Auflagerung an Innenfläche Mandibula (II.) und Außenseite Os sphenoidale (II.), Auflagerungen an Humerus (II.), Os metacarpale (II.), Becken (II.), Rippen (II.), Clavicula(II.)

= Merkmale 2 x I., 11 x II. = an Skorbut erkranktes Kind 
2). Grab 72: $\quad$ (s. auch IV.8.2)

hämorrhagisch bedingte Auflagerungen auf der Lamina externa des Os frontale (I), hämorrhagisch bedingte Auflagerungen an Alveolarrändern Maxilla (I.) und Mandibula (I.), histologisch nachgewiesene, hämorrhagisch bedingte Cribra orbitalia (I.), atypische Gefäßimpressionen Lamina interna des Schädeldaches (II.), Dorsalseite Maxilla porös (II.), Außenseite Os sphenoidale und Umgebung porös (II.), Umgebung des Foramen infraorbitale porös (II.)

= Merkmale 4 x I., 4 x II. = an Skorbut erkranktes Kind

3). Grab 6 B:

hämorrhagisch bedingte Auflagerungen an Alveolarrändern der Maxilla (I.),

Hinterfläche von Maxilla (II.) und Processus zygomaticus (II.) porös, Stomatitis (II.), Außenseite des Os sphenoidale und Umgebung porös (II.), Auflagerung auf Ala minor (II.) und pars petrosa (II.) ossis sphenoidalis

= Merkmale 1 x I., 6 x II. = an Skorbut erkranktes Kind

4). Grab 279 A:

hämorrhagisch bedingte Auflagerungen an Alveolarrändern der Maxilla (I.), Osteophyten-

bildungen im Bereich der Metaphysen der langen Röhrenknochen (I.),

Cribra orbitalia (ohne Histologie) (II.), Auflagerung an distaler Ulna (II.)

= Merkmale 2 x I., 2 x II. = Verdacht auf Skorbut

5). Grab 164:

hämorrhagisch bedingte Auflagerungen an Alveolarrändern der Mandibula (I.),

Stomatitis (II.), Cribra orbitalia (ohne Histologie) (II.)

= Merkmale 1 x I., 2 x II. = kein Skorbut

Medizinischer Hintergrund:

Die Ursache eines Skorbuts ist der chronische Mangel an Vitamin C (= Ascorbinsäure). Da der Mensch im Gegensatz zu anderen Lebewesen Ascorbinsäure nicht selber synthetisieren kann, ist er auf eine ausreichende Auf- 
nahme mit der Nahrung (vor allem in frischem Obst, Gemüse, Fleisch und Milch) angewiesen. Dem Körper ist es bei unzureichender Zufuhr nicht möglich ist die Aminosäuren Prolin und Lysin im Rahmen der Kollagenbiosynthese zu hydroxylieren. Als Folge ist die Tripelhelixbildung der Kollagenfibrillen nicht möglich und es entsteht ein minderwertiges, weniger stabiles Kollagen. Gewebe, die zu großen Teilen aus Kollagen bestehen, sind folglich besonders betroffen. Das trifft vor allem auf Knochen und Bindegewebe zu. Es resultiert zum einen eine primäre, typische Störung des Knochenwachstums mit typischen Veränderungen der enchondralen Knochenformationen. Der schnellwachsende Knochen der costochondralen Übergänge und der epi-diaphysealen Regionen der Langknochen sind besonders betroffen (JAFFE 1972, ORTNER 2003, FRAENKEL 1908). Zum anderen treten aufgrund der Brüchigkeit von Gefäßwänden multiple Einblutungen in verschiedene Gewebe auf. Prinzipiell sind Einblutungen überall möglich, bevorzugt treten sie jedoch in Schleimhäute, den Zahnhalteapparat und unter das Periost der Knochen auf (BARLOW 1895 a und 1895 b, FRAENKEL 1908, HART \& LESSING 1913).

\section{$\underline{\text { III.5.2 Anämie }}$}

Eine beginnende Anämie tritt bei vier von 47 der Kinder ( $=$ 4/ 47: 8,5\%) auf (Gräber 72, 81, 86 und 113). Bei diesen Kindern sind im Bereich der Lambdanaht poröse Oberflächen zu beobachten. Wird nur das Schädeldach betrachtet, sind sogar 9,8 \% ( $n=4 / 41:$ 9,8 \%) der Kinder betroffen. Bei zwei weiteren Kindern (Gräber 299 und 318) kann eine Anämie vermutet werden ( $n=2 / 41: 4,25 \%$ ). Differentialdiagnostisch ist auch bei dem Kind aus Grab 340 an eine Anämie zu denken (s. Katalog). Bei dem Kind aus Grab 81 zeigt sich mikroskopisch stellenweise eine Ausdünnung der Lamina externa mit einer begleitenden Hypertrophie der Diploë (s. III.2.2). Das Erscheinungsbild ist nicht ganz typisch für eine Anämie, allerdings finden sich auch keine Hinweise auf andersartige Erkrankungen. Es handelt sich wahrscheinlich um ein sehr frühes Stadium einer Anämie (Tafel 2). Außer den Veränderungen am Schädeldach weist das Kind aus Grab 72 in der Histologie auch anämietypische Veränderungen am Orbitadach auf (s. III.2.6. und IV.8.2) (Tafeln 12 und 13). Das Kind aus Grab 299 zeigt am Orbitadach möglicherweise anämieverdächtige Veränderungen (Tafel 16) (vgl. III.2.6 und III.4.3). 
Medizinischer Hintergrund:

Eine Anämie ist nur ein Symptom das bei unterschiedlichsten Erkrankungen auftreten kann. Im Folgenden sind einige der wichtigsten Ursachen aufgeführt. Dabei kann grundsätzlich zwischen angeborene und erworbene Anämien unterschieden werden. Es gibt eine Vielzahl von angeborenen Anämien, die alle sehr selten sind. Auch die Ursachen erworbener Anämie sind vielfältig (KRIENER et al. 2001). Einige häufige Anämieformen werden hier angeführt:

Mangelanämien bei Minderversorgung von z.B. Eisen, Proteinen, Vitaminen (B12, C, Folsäure)

Blutungsanämien bei rezidivierenden und chronischen Blutungen z.B. im Rahmen entzündlicher

Darmerkrankungen, Parasitenbefall des Darmtraktes, Skorbut

Infektanämien bei schweren akuten oder chronischen Erkrankungen

Tumoranämien bei malignen Erkrankungen

Merkmal einer Anämie ist eine Expansion des roten Knochenmarkes. Sie stellt den Versuch der Kompensation des Mangels an Erythrozyten durch Vergrößerung des Produktionsraumes dar (FISCHER 1974, SCHULTZ 2001 a, RITTER 2001). Dieses zeigt sich makroskopisch durch eine verstärkte Porosität des Schädeldaches. Meist beginnt die poröse Ausdünnung der Lamina externa in der Nähe der Lambdanaht an den Ossa parietalia oder am Os occipitale. Es kann bis zu vollständigen Auflösung der Lamina externa kommen (SCHULTZ 2001 b und 1989 c, CARLI-THIELE \& SCHULTZ 2001). Mikroskopisch zeigt sich eine Erweiterung der Markräume (SCHULTZ 2001 b). Neugebildete Spongiosabälkchen verlaufen parallel zueinander und senkrecht zur Knochenoberfläche (SCHULTZ 2001 b, WAPLER et al. 2004). Gleichartige Veränderungen sind häufig auch als Cribra orbitalia am Orbitadach (GÖTZ 1988, SCHULTZ 2001 b) zu finden. Weitere Merkmale einer Anämie können verplumpte, angeschwollene und verdickte Schulterblätter, Becken, Rippen und Wirbelkörper sein. Zwischen den verschiedenen Anämieformen kann am mazerierten Knochen mikroskopisch nicht differenziert werden (WAPLER et al. 2004). 
III.5.2.1 Merkmale einer Anämie

Tab. 10 Merkmale einer Anämie am mazerierten Knochen (CARLI-THIELE 1996, ORTNER 2003, SCHULTZ 2001 b und 2003, WAPLER et al. 2004)

\begin{tabular}{|l|l|l|}
\hline $\begin{array}{l}\text { betroffene } \\
\text { Strukturen }\end{array}$ & Lokalisation der Veränderungen & Art der Veränderungen \\
\hline L. externa & $\begin{array}{l}\text { gesamte Kalotte, } \\
\text { Beginn bds. der Lambdanaht }\end{array}$ & $\begin{array}{l}\text { verstärkte Porosität bis zu massiven } \\
\text { Knochenneubildungen, } \\
\text { "Bürstenschädel" }\end{array}$ \\
\hline Orbita & Orbitadach & $\begin{array}{l}\text { verstärkte Porosität bis zu massiven } \\
\text { Knochenneubildungen }\end{array}$ \\
\hline Scapula & & verplumpt \\
\hline Becken & gesamter Knochen & angeschwollen \\
\cline { 1 - 1 } Rippen & & verdickt \\
\hline Wirbelkörper & & \\
\hline
\end{tabular}

\section{$\underline{\text { III.5.3 Rachitis }}$}

Rachitis lässt sich bei keinem Kind der Mad'arovce-Kultur nachweisen ( $\mathrm{n}=0$ / 58). Lediglich bei einem Kind (Grab 102) zeigen sich am Schädeldach histologische Veränderungen der Diploë, die Hinweise auf eine ausgeheilte Rachitis geben ( $=1 /$ 58: 1,7 \%) (s. III.2.2). Das Schädeldach dieses Kindes ist nicht normal strukturiert. Die Markräume sind verkleinert, die Trabekel sind grazil und irregulär, parallel zur Oberfläche angeordnet (Tafel 3, s. III.2.2).

Medizinischer Hintergrund:

Der Rachitis liegt ein Vitamin-D-Mangel im Kindesalter zugrunde. Das Fehlen von Vitamin D führt zu einer Verminderung der Calcium- und Phosphatresorption sowie einer Störung der Hydroxylapatitsynthese. Von der Erweichung und der abnormen Biegsamkeit des Knochens aufgrund der unzureichenden Mineralisation des neugebildeten Osteoids (FRAENKEL \& LOREY 1910, ORTNER 2003) sind vor allen die schnellwachsenden Knochen (lange Röhrenknochen, Knorpel-Knochengrenzen Rippen) betroffen (ORTNER 2003, WIMBERGER 1925). Anders als Vitamin C kann Vitamin D vom menschlichen Körper unter bestimmten Voraussetzungen synthetisiert werden. Bei einem Teilschritt der Vitaminsynthese in der Epidermis wird UV-Licht benötigt. Deswegen entsteht eine Rachitis vor allem aufgrund ungenügender Exposition der Haut gegenüber dem Sonnenlicht. 


\section{III.5.3.1 Merkmale einer Rachitis}

Tab. 11 Merkmale einer Rachitis am mazerierten Knochen (FRAENKEL \& LOREY 1910,

KLOSE 1919, KÜBler 1974, ORTNER \& MAYs 1998, ORTNER 2003, SCHUltZ 2001 b und 2003)

\begin{tabular}{|l|l|l|}
\hline $\begin{array}{l}\text { betroffene } \\
\text { Strukturen }\end{array}$ & $\begin{array}{l}\text { Lokalisation der } \\
\text { Veränderungen }\end{array}$ & Art der Veränderungen \\
\hline L. externa & vor allem Tubera frontal und & rachitischer Osteophyt, "Quadratschädel" \\
vor allem bds. der Lambdanaht & Kraniotabes \\
verzögerter Fontanellenschluss
\end{tabular}


Abb. 16 Das Auftreten der verschiedenen Skorbutmerkmale bei den skorbutkranken Kindern der Mad'arovce-Kultur.

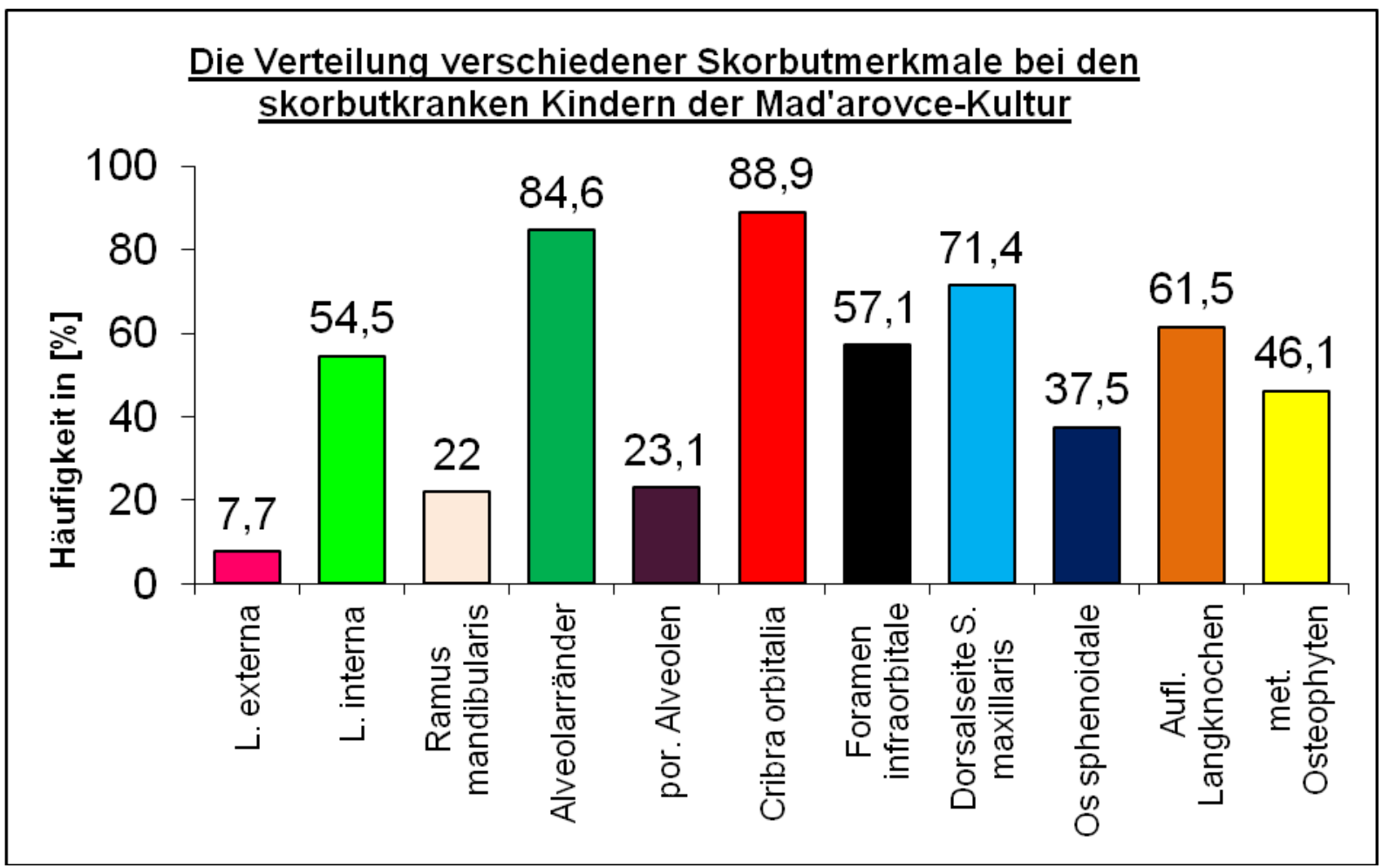

Abb. 17 Das Auftreten der verschiedenen Skorbutmerkmale bei den skorbutkranken Kindern der Mad'arovce-Kultur in abfallender Häufigkeit.

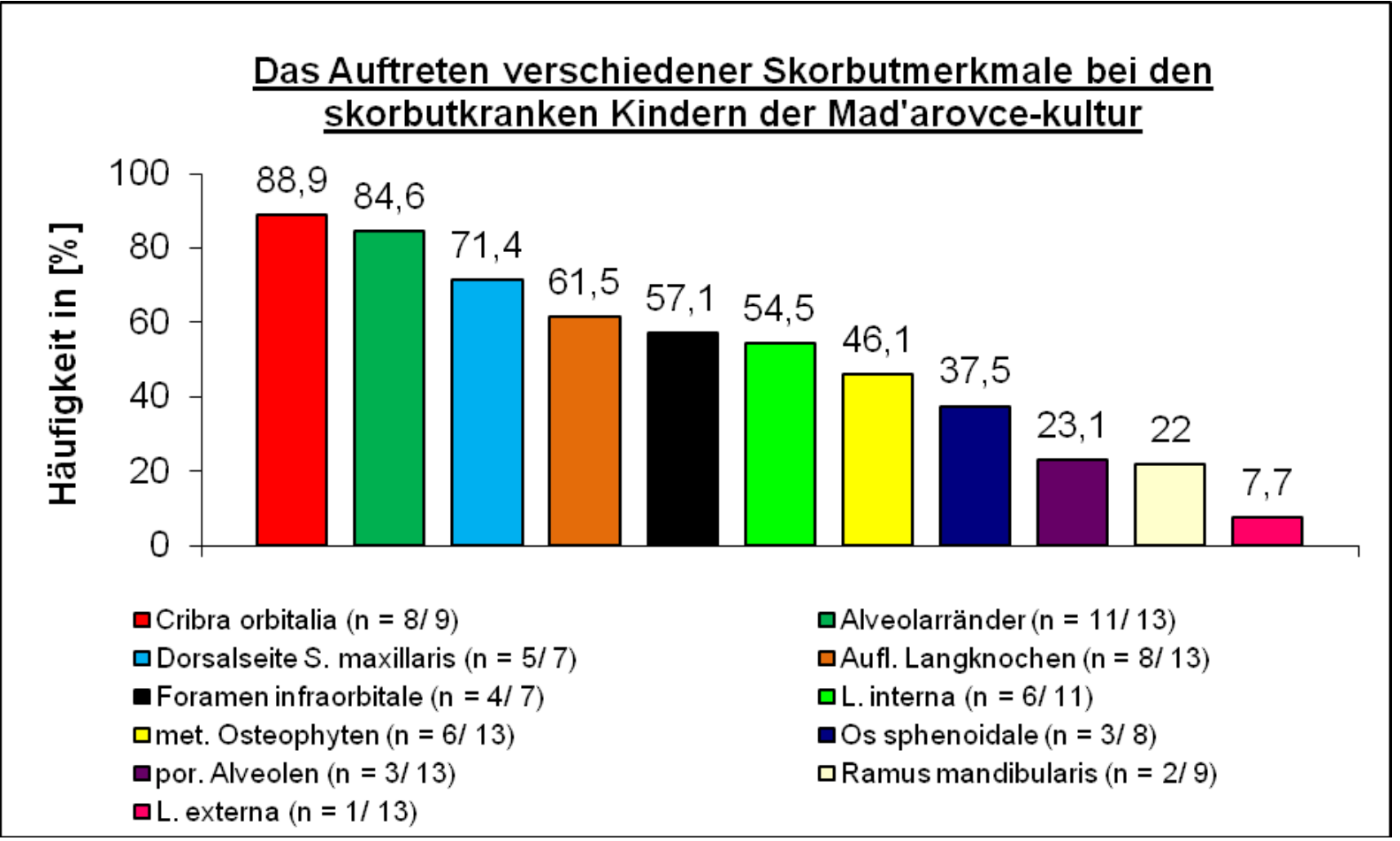

( S.: Sinus, Aufl.: Auflagerung, L.: Lamina, met.: metaphysäre, por.: porös ausgekleidete) 


\section{III.6 Charakterisierung des Gesundheitsstatus der Kinder der Mad'arovce-}

$\underline{\text { Kultur }}$

Im folgende Abschnitt wird der Gesundheitsstatus der Kinder der Mad'arovce-Kultur von Jelšovce charakterisiert, indem eine Übersicht über auftretenden Erkrankungen gegeben wird. Endocranial treten Spuren meningealer Reizungen $(n=12 / 45$ : 26,7 \%) und Entzündungen im Bereich der venösen Hirnblutleiter (Perisinusitis: $n=11 / 42$ : 26,2 \%) auf (Abb. 38). Mikroskopisch stellt sich bei drei Kindern heraus, dass die meningealen Reizungen hämorrhagischen Ursprungs sind ( $n=3 / 12$ : $25 \%$ ). Bei 34,8 \% der Kinder findet sich eine Hirndrucksymptomatik ( $\mathrm{n}=16 / 46)$. Die Erkrankungen der Nasenhöhle und der Nasennebenhöhlen verteilen sich wie folgt (Abb. 38): Rhinitis: $n=8 / 20: 40 \%$; Sinusitis maxillaris: $n=6$ / 17: 35,3 \%; Sinusitis frontalis: $n=2 / 7: 28,6 \%$; Sinusitis sphenoidalis: $n=$ 1/3: 33,3 \%; Sinusitis ethmoidalis: 0/ 4: 0 \%. Von einer Otitis media sind drei von 24 (n = 3/

24: 12,5\%), von einer Hemmung der Pneumatisation des Warzenfortsatzes sechs von 33 Kindern ( $n=6 / 33$ : 18,2\%) betroffen (Abb. 38). Ein Kind litt an einer Mastoiditis ( $n=1 / 33$ : 3\%) (Abb. 38). Cribra orbitalia zeigen sich bei 62,5 \% der Kinder $(n=20 / 32)$ (Abb. 39).

Eine Pleuritis tritt bei den Kindern der Mad'arovce-Kultur nicht auf ( $n=0 / 31$ : 0 \%) (Abb. 39). Bei zwei Kindern wurde eine Spina bifida, bei drei Kindern eine Skoliose diagnostiziert. Enthesiopathien durch körperliche Überanstrengung treten an Claviculae ( $\mathrm{n}=4$ / 27: 14,8 \%) und Tibiae ( $\mathrm{n}=11$ / 33: 33,3 \%) auf. Ehemalig subperiostal gelegene plattenartige Auflagerungen auf den langen Röhrenknochen sind bei 48,6 \% $(n=17 / 35)$ der Kinder nachzuweisen (Abb. 39): Die oberen Extremitäten und der Schultergürtel sind zu 17,1 \% ( $n=6 / 35)$, die unteren Extremitäten zu 45,5 \% $(n=15 / 33)$ betroffen. Bei sechs von 29 Kindern $(n=6 / 29$ : 20,7 \%) treten skorbutbedingt Osteophytenbildungen im Bereich der Metaphysen der langen Röhrenknochen im Sinne einer „Krempelung “ der Metaphysenplatte auf (Abb. 39). An einer Mangelerkrankung leiden 66,1 \% der Kinder $(n=39 / 59)$. Im Einzelnen sind 24,4 \% $(n=12 /$ 49) von Skorbut, 0\% ( $=0 / 58)$ von Rachitis und 8,5 \% (n= 4/47) von Anämie betroffen 
(Abb. 39). Bei einem Kind ist eine ausgeheilte, klinisch nicht mehr bestehende Rachitis nachweisbar $(\mathrm{n}=1 /$ 58: 1,7 \%). Harris-Linien treten bei 89,5 \% $(\mathrm{n}=17 / 19)$ der Kinder auf (Abb. 39). Transversale Schmelzhypoplasien sind bei 60,8\% $(n=31 / 51)$ der Kinder zu beobachten (Abb. 39). Transversale Zementhypoplasien treten bei drei Kindern auf. Bei 35,7 \% $(n=10 / 28)$ der Kinder zeigt sich eine Stomatitis. Bei 6,1\% $(n=3 / 49)$ ist eine Karies, bei 56,3 $\%(n=27 / 48)$ Zahnstein nachweisbar (Abb. 39). An Parodontose litten 63,4\% (n = 26/ 41) der Kinder, von diese war die Hälfte ebenfalls an Parodontitis erkrankt $(n=13 / 41$ : 31,7 \%) (Abb. 39).

Die Art und Häufigkeit der Erkrankungen bei den Kindern der Mad'arovce-Kultur charakterisieren diese Population als Angehörige einer sesshaften Bauernpopulation.

Abb. 38 Häufigkeitsverteilung verschiedener Erkrankungen des Craniums

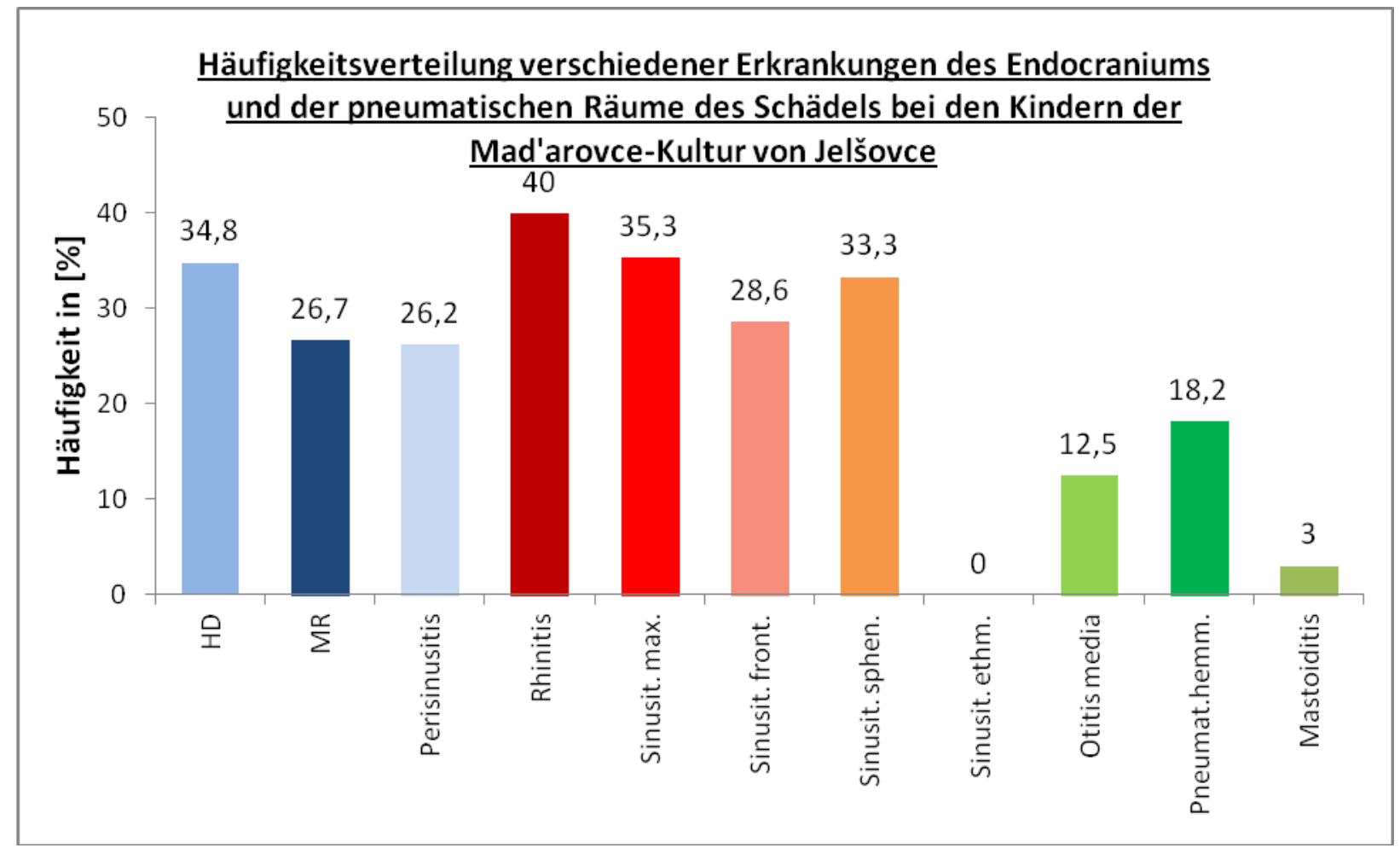

(HD: Hirndrucksymptomatik, MR: meningeale Reizungen, Sinusit.: Sinusitis, max.: maxillaris, front.: frontalis, sphen.: sphenoidalis, ethm.: ethmoidalis, Pneumat.hemm.: Pneumatisationshemmungen) 
Abb. 39 Häufigkeitsverteilung verschiedener Erkrankungen bei den Kindern der Mad'arovceKultur von Jelšovce

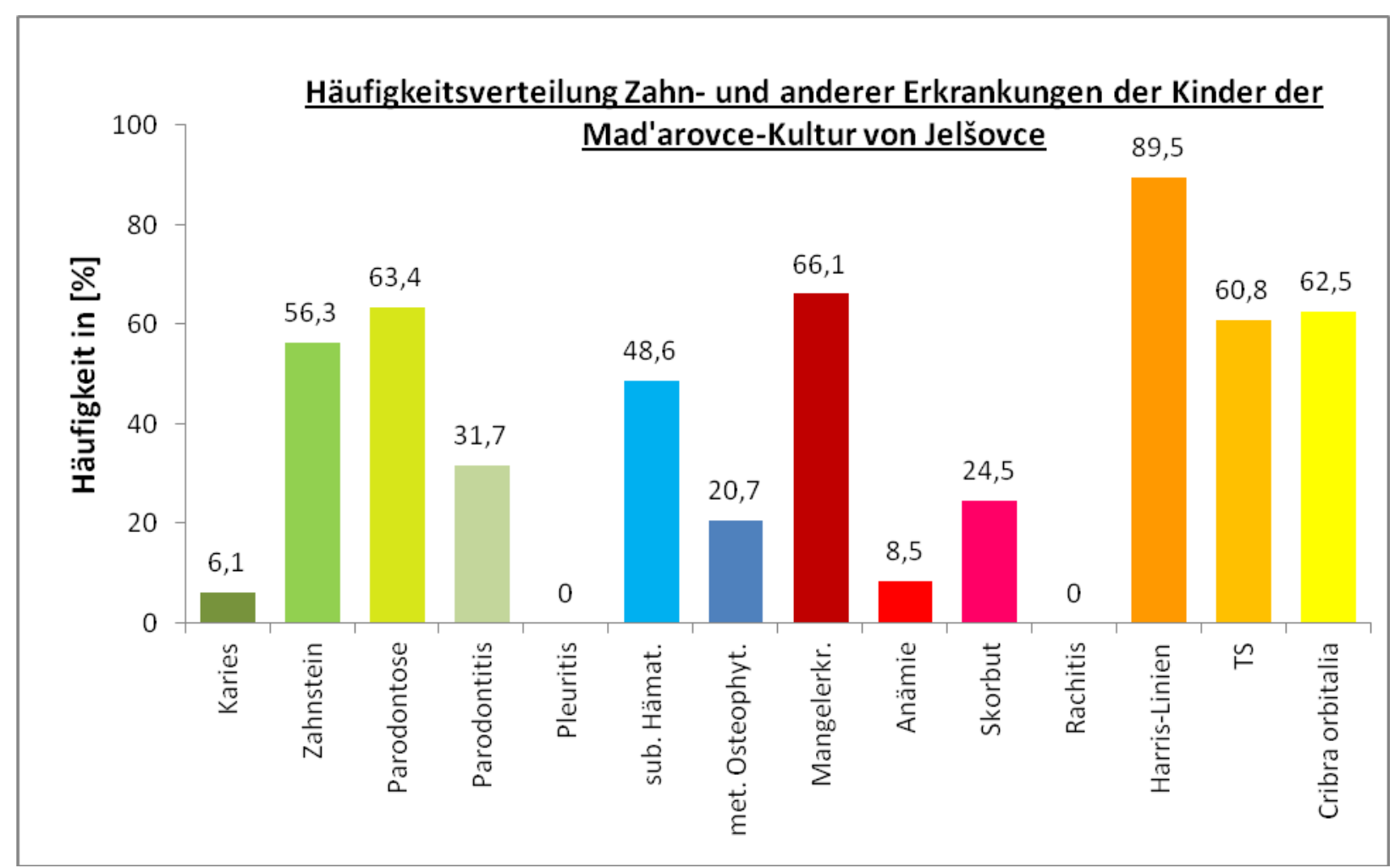

(sub. Hämat.: subperiostale Hämatome, met.: metaphysäre, Osteophyt.: Osteophyten,

Mangelerkr.: Mangelerkrankungen, TS: transversale Schmelzhypoplasien) 


\section{$\underline{\text { IV. Diskussion }}$}

\section{IV.1. Vergleichspopulationen}

Die Ergebnisse der Untersuchungen an den Kinderskeleten der Mad'arovce-Kultur sollen mit denen anderer Kulturen verglichen werden um Unterschiede in Lebensbedingungen zu erkennen. Bei der Suche nach Vergleichsdaten ist zu berücksichtigen, dass die veröffentlichen Ergebnisse nicht immer nach den gleichen Untersuchungsmethoden erhoben wurden.

Verschiedene Autoren haben unter Umständen unterschiedliche Kriterien und Einteilungen zu Altersbestimmung und Krankheitsmerkmalen verwendeten, die zum Teil in ihrem Ergebnis etwas variieren und nicht immer aus den Veröffentlichungen eindeutig zu erschließen sind. Deshalb wird in dieser Arbeit bevorzugt auf Skeletserien zurückgegriffen, die in der Arbeitsgruppe Paläopathologie in Göttingen nach den gleichen Richtlinien untersucht wurden. Ein besonderes Augenmerk wird dabei auf die von demselben Gräberfeld in Jelšovce stammenden Kinderskelete der Nitra- und Aunjetitz-Kultur gelegt. Diese wurden in den Jahren 1993 und 1996 von Professor Dr. Dr. M. Schultz untersucht (SCHUltz 2001 a, ScHULTZ et al. 1998, BÁTORA \& SCHULTZ 2001, SCHULTZ \& SCHMIDT-SCHULTZ 1999).

Weitere Populationen, die zu Vergleichen herangezogen wurden:

\section{Bronzezeit:}

Traisental, Niederösterreich:

Hainburg: $\quad$ 1800-1500 v. Chr.

Wieselburger-Kultur (SCHULTZ 1988/89 \& 2001 a)

Franzhausen-I : $\quad$ 2200-1800 v. Chr.

Unterwölblinger-Kultur (SCHULTZ 1987, 1988/89, 1989 a \& 2001 a)

Pottenbrunn 2200-1900 v. Chr.

Unterwölblinger-Kultur (SCHULTZ 1987, 1989 a \& 2001 a) 
Gemeinlebarn-F 2200-1800 v. Chr.

Věteřov-Kultur (SCHULTZ 1987, 1988/89, 1989 a \& 2001 a)

Pitten 1500-1250 v. Chr.

Mittelbronzezeit $\quad$ (SCHULTZ 1987, 1988/89, 1989 a \& 2001 a)

Anatolien:

İkiztepe 2500-2300 v. Chr.

Frühbronzezeit $\quad$ (SCHULTZ 1988/89, 1989 a, 1990 \& 2001 a)

\section{Neolithikum:}

Wandersleben Linienbandkeramik (CARLI-THIELE 1996, CARLI-THIELE \&

SCHULTZ 2001, BRAULKE 2004)

Aiterhofen Linienbandkeramik (CARLI-THIELE 1996, CARLI-THIELE \&

SCHULTZ 2001)

\section{$\underline{\text { Mittelalter: }}$}

$\begin{array}{lll}\begin{array}{ll}\text { Barbing } \\ \text { 5traubing }\end{array} & \text { 5.-6. Jh. n. Chr. } & \text { (DETKEN 1989) } \\ \text { Harting } & \text { 6.-8. Jh. n. Chr. } & \text { (KREUTZ 1997) } \\ \text { Boğazkale } & \text { 6.-9. Jh. n. Chr. } & \text { (PCHL 1989) } \\ & & \text { SCHULTZ 1989 b, SCHULTZ \& SCHMIDT- } \\ \text { Bettingen } & \text { 12. Jh. n. Chr. } & \text { (TEMPLIN 1993) } \\ \text { Pergamon } & \text { 13.-14. Jh. n. Chr. } & \text { (SCHULTZ 1989 b, SCHULTZ \& SCHMIDT- } \\ & & \end{array}$

Wird in den folgenden Texten auf die hier genannten Populationen Bezug genommen, so wurden die Vergleichsdaten, wenn nicht anders vermerkt, aus diesen Veröffentlichungen entnommen. 


\section{IV.1.1 Gemeinsamkeiten und Unterschiede der verglichenen Populationen}

Um die festgestellten Unterschiede der Vergleichsergebnisse richtig interpretieren zu können, sollen zunächst Ähnlichkeiten und Unterschiede in Lebensweise und Umweltbedingungen zwischen den verschiedenen Populationen betrachtet werden (vgl. Tab. 12 und 13).

Den drei Populationen aus Jelšovce ist ihr Lebensraum gemeinsam. Die geographischen Voraussetzungen waren die gleichen. Sie nutzten die gleichen natürlichen Ressourcen und landwirtschaftlichen Flächen und lebten hauptsächlich vom Ackerbauern, betrieben aber auch Viehzucht, Metallverarbeitung und Töpferei. Zahlreiche Kontakte zu benachbart lebenden Menschen anderer Kulturkreise sind für alle drei Kulturen belegt. Unterschiede bestehen in der Populationsgröße, der absoluten Datierung und der ethnischen Zugehörigkeit. Die Mad'arovce-Kultur ist die jüngste und größte Population (79 Kinderskelete). Ihre Träger sind genetisch mit denen der Aunjetitz-Kultur verwandt. Bei der Aunjetitz-Kultur handelt es sich um die kleinste Population (48 Kinderskelete). Die Nitra-Kultur ist die älteste der drei Populationen, liegt von der Größe zwischen den beiden Anderen (58 Kinderskelete) und unterscheidet sich genetisch von diesen. Alle drei Kulturen können als relativ reich gelten. Der Wohlstand nahm im Verlauf der Frühbronzezeit sogar noch zu. Ein Zeichen dafür sind die lediglich aus der Mad'arovce-Kultur stammenden Funde von Gold- und Bleischmuckstücken. Die Menschen der Mad'arovce-Kultur lebten in einer kleinen befestigten Siedlung nahe des Flusses. Aus Aunjetitz- und Nitra-Kultur wurden in Jelšovce bisher zwar keine Siedlungen bekannt, aber es kann wohl davon ausgehen werden, dass sie ebenfalls in kleinen Dörfern in Flussnähe lebten.

Im Verlauf der frühen Bronzezeit kam es zu wirtschaftlichen und gesellschaftlichen Veränderungen (BEHRINGER 2007). Gesellschaftliche Veränderungen zeigen sich unter anderem an der Struktur des Gräberfeldes. Bei etwa gleichlanger Belegungsdauer (jeweils circa 200 Jahre) nehmen die Gräber der Mad'arovce-Kultur die weitaus größte Fläche ein. Während in Aunjetitz- und Nitra-Kultur überwiegend in Reihen angeordnet sind, kommt es in der Mad'arovce- 
Kultur zu in Gruppen angeordnete Bestattungen mit größeren Distanzen untereinander. Zum Teil treten Nachbestattungen in den Grabhügeln reicher, zentral gelegenen Gräber auf (vgl. BÁTORA 2000). Familiären Beziehungen scheinen als Zeichen geänderter Machtstrukturen wichtiger zu werden. Die wirtschaftlichen Veränderungen zeichnen sich durch eine zunehmende Spezialisierung in Handwerk, Metallurgie und Handel aus (Handwerker - Händler Bauern). Zum Beispiel wurden die Herstellung und Verwendung von Bronze anstatt von Kupfer üblich. Durch Verbesserung der Kenntnisse konnten härtere, haltbarere Legierungen hergestellt werden (BEHRINGER 2007). Auch die landwirtschaftlichen Bedingungen änderten sich im Laufe der Frühbronzezeit durch langsamen Klimawandel und jahrhundertelange Nutzung der gleichen Anbauflächen. Klimatische Veränderungen während der Frühbronzezeit sind aus der Forschung bekannt (BOUZEK 1982, 1993 und 2001, FURMÁNEK et al. 1999, BEHRINGER 2007) (Abb. 32). Im Äneolithikum herrschte während des Atlantikums in Mitteleuropa ein sehr stabiles, feucht warmes Klima, durchschnittlich etwa 3 bis $4{ }^{\circ} \mathrm{C}$ wärmer und 60 bis $70 \%$ feuchter als heute. Die Frühbronzezeit fällt in die Endphase des Atlantikums, das sogenannte Epiatlantikum. Es kam es zu einer mäßigen Abkühlung (FURMÁNEK et al. 1999). Kürzere feuchte und kürzere trockene Perioden wechselten in rascher Folge. Charakteristisch ist vor allem eine Instabilität des Klimas mit ausgeprägten Klimaschwankungen innerhalb weniger (10 - 20) Jahre. Für die drei frühbronzezeitlichen Populationen aus Jelšovce lässt sich feststellen, dass alle drei in einer Zeit mit starken Klimaschwankungen lebten. Es lässt sich postulieren, dass zur Zeit der Nitra-Kultur das Klima eher feuchter und wärmer war als zur Zeit der Mad'arovce-Kultur. Diese Klimaveränderungen lassen sich auch anhand Veränderungen des Bodens der Grabverfüllungen erkennen. In Aunjetitz- und Nitra-Kultur handelt es sich um Löß. In den Verfüllungen der mad'arovcezeitlichen Gräbern finden sich Schwarz- und Parabraunerden. Schwarz- und Braunerden entstehen durch Degradation aus Lößböden. Während die sehr fruchtbaren Löß- und Schwarzerdeböden Niederschläge über längere Zeit speichern und die Vegetation so längere Trockenperioden ohne Schaden überstehen kann, 
verlieren Schwarz- und Braunerden diese Eigenschaft (SCHEFFLER \& SCHACHTSCHABEL 2002). Den Menschen der Nitra- und der Aunjetitz-Kultur scheinen insgesamt hochwertigere Ackerböden zur Verfügung gestanden zu haben als denjenigen der Mad'arovce-Kultur. Zudem mussten die Menschen der Mad'arovce-Kultur auch mehr Mitglieder der Gemeinschaft von diesen weniger fruchtbaren Böden versorgen. Allerdings kann die Beackerung die Degradierung der Böden verzögern (SCHEFFLER \& SCHACHTSCHABEL 2002). Die landwirtschaftlichen Voraussetzungen waren somit auch für die Mad'arovce-Kultur nicht schlecht. Noch heute findet man in der Gegend um Jelšovce sehr fruchtbares Ackerland.

Die niederösterreichischen Populationen des Traisentals hatten ähnliche wirtschaftliche und gesellschaftliche Grundlagen wie die Populationen aus Jelšovce. Wie Jelšovce waren Franzhausen, Pottenbrunn und Gemeinlebarn vom Neolithikum bis in das Mittelalter fast durchgängig besiedelt (NEUGEBAUER 1994 und 1997). Die in der Frühbronzezeit dort lebenden Menschen betrieben neben der Landwirtschaft auch Viehzucht und Metallurgie. Ihre kleinen Dörfer befanden sich in Flussnähe. In Franzhausen konnten einzelne, locker gereihte Gehöfte und Weiler mit mehreren Gebäuden, Langhäusern und kleineren Gebäuden entlang des Flusslaufes gefunden werden. Pro Gehöft lebten etwa acht bis zwölf Personen (NEUGEBAUER 1994 und 1997). Daraus ergibt sich eine Gruppe von 25 bis 30 Personen je Generation (BERNER 1997). Bei der Franzhausener Population (Franzhausen-I) handelte es sich um überdurchschnittlich reiche Ackerbauern, wie sich anhand von Grabbeigaben erkennen lässt (NEUGEBAuer 1994, Neugebauer-MAREsCH \& Neugebauer 1988). Die Bevölkerungen von Pitten, Hainburg und Gemeinlebarn-F hingegen zeichneten sich nicht durch besonderen Reichtum aus, allerdings wurden auch zahlreiche reich ausgestattete Gräber gefunden (SCHULTZ 1989 a).

Die Hainburger Population unterscheidet sich von den übrigen niederösterreichischen Populationen. Sie gehört einem anderen Kulturkomplex, der östlich des Wiener Waldes angesiedelten Wieselburger Kultur an. Ihr Siedlungsgebiet liegt im Grenzgebiet der heutigen Staaten 
Österreich, Ungarn und Slowakei. Die wirtschaftlichen Grundlagen ähneln aber denen der anderen niederösterreichischen Populationen (SCHULTZ 1988/89). Es bestanden Handelskontakte zu diesen benachbarten Kulturen. Importwaren aus Aunjetitz-, Mad'arovceund Věteřov-Kultur in Form von Tassen und Amphoren wurden gefunden (FURMÁNEK et al. 1999).

Die Population von İkiztepe unterscheidet sich in verschiedenen Punkten von den übrigen bronzezeitlichen Populationen. Die anatolische Siedlung von İkiztepe liegt auf vier Hügeln an der Schwarzmeerküste an der Mündung des Kizilirmak (antiker Halys). Die Bevölkerung bestand aus Bauern und Fischern, die einen ausgedehnten Handel mit ihren Nachbarn betrieben. Die Belegungszeit des frühbronzezeitlichen Gräberfeldes beträgt etwa 200 Jahre. Aus der Anfangsphase sind Pithosbestattungen in Siedlungen von Säuglingen und Kleinkindern bis zu drei Jahren bekannt (WITTWER-BACKOFEN 1997). Absolut gesehen ist die Siedlung etwas älter als die verglichenen bronzezeitlichen Populationen in Mitteleuropa. Aufgrund dessen fand auch kein direkter kultureller Austausch statt. Auch vom Biotop her unterscheiden sich die im Landesinneren gelegenen Populationen in Niederösterreich und Jelšovce von dem am Schwarzen Meer gelegenen İkiztepe. Daraus ergibt sich auch ein Unterschied in den zur Verfügung stehenden Nahrungsmitteln. Die anatolische Population von İkiztepe kann aufgrund von Grabbeigaben als überdurchschnittlich reich bezeichnet werden (BILGI 1984).

Die Linienbandkeramiker aus Wandersleben lebten in Mitteldeutschland in der letzten Periode der Steinzeit, die sich durch Sesshaftigkeit, Viehzucht, Ackerbau und Töpferei auszeichnete. Sie lebten in großen Langhäusern, die Wohnung, Stall und Speicher unter einem Dach vereinten (BRAULKE 2004, CARLI-THIELE 1996). Klimatisch gesehen lebten sie im Atlantikum, d.h. insgesamt war das Klima stabiler, wärmer und feuchter als zu Zeiten der bronzezeitlichen Kulturen. Wie in İkiztepe ergibt sich auch hier nicht die Möglichkeit eines direkten kulturellen Austausches. 
Tab. 12 Gemeinsamkeiten und Unterschiede der Populationen der Nitra-, Aunjetitz- und Mad'arovce-Kultur von Jelšovce (BÁTORA 2000, BÁTORA \& SCHULTZ 2001)

\begin{tabular}{|c|c|}
\hline Gemeinsamkeiten & Unterschiede \\
\hline Frühbronzezeit & absolute Datierung \\
\hline Ackerbau und Viehzucht & ethnische Zugehörigkeit \\
\hline gleiches Siedlungsgebiet & Populationsgröße \\
\hline gleichen Anbauflächen & Bodenbeschaffenheit \\
\hline relativ reich & Bronze statt Kupfer \\
\hline Belegungszeit des Gräberfeldes & zunehmende Spezialisierung \\
\hline Klimawandel & Verteilung der Krankheitshäufigkeiten \\
\hline & Befestigung der Siedlungen \\
\hline
\end{tabular}

Tab. 13 Vergleiche der Lebensbedingungen zwischen den untersuchten Populationen aus Niederösterreich und Jelšovce, Wandersleben und İkiztepe (BÁTORA 2000, BÁTORA \& SCHUltz 2001, BiLgi 1984, BRAulKe 2004, CARLi-ThIELE 1996, SCHUltZ 1990)

\begin{tabular}{|l|l|l|}
\hline Niederösterreich und Jelšovce & Wandersleben & İkiztepe \\
\hline Biotope im Landesinneren & Landesinnere & Schwarzmeerküste \\
\hline Flusstäler / flussnah & & flussnah \\
\hline Epiatlantikum & Atlantikum & Atlantikum - Epiatlantikum \\
\hline Kupfer und Bronze & Stein & Kupfer und Bronze \\
\hline kulturelle Kontakte untereinander & & \\
\hline Ackerbau, Viehzucht & Ackerbau, Viehzucht & Ackerbau, Viehzucht und \\
\hline gegenseitige Beeinflussung & & Fischfang \\
\hline zahlreiche Handelskontakte zu & & zahlreiche Handelskontakte \\
\hline benachbarten Kulturen & & zu benachbarten Kulturen \\
\hline
\end{tabular}


Abb. 32 Diagramm der Klimaschwankungen zwischen Neolithikum und

Völkerwanderungszeit (nach BOUZEK 1982, S. 189)

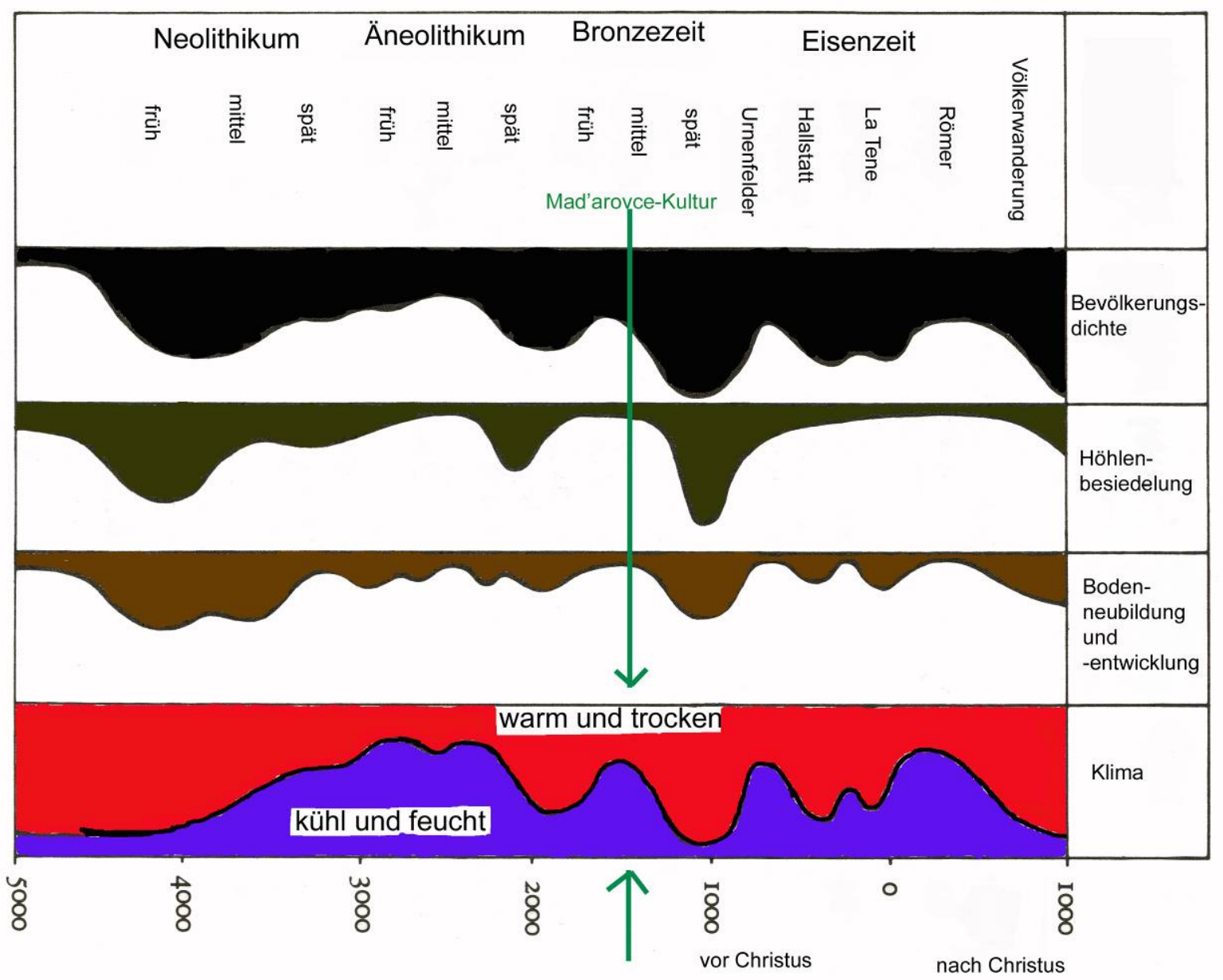

Die Abb. 32 zeigt, dass die Kinder der Mad'arovce-Kultur mit einem eher kühler und feuchteren Klima leben mussten als die Kinder der vorrausgegangenen Kulturen in Slowakei und Niederösterreich. Seit der Zeit der Nitra-Kultur hatte sich das Klima zunehmend abgekühlt. Die Bevölkerungsdichte nahm in dem gleichen Zeitraum leicht zu. Wenig Bodenneubildung und eine geringe Besiedlung von Höhlen sind ebenfalls ein Zeichen des kühler und feuchter werdenden Klimas (s. BOUZEK 1982, S. 189).

\section{IV.2. Altersverteilung}

\section{IV.2.1 Kleinkinderdefizit}

Das bekannte Problem der fehlenden Feten- und Kleinkinderskelete auf prähistorischen Gräberfeldern (Kleinkinderdefizit) zeigt sich auch in der Altersverteilung der Kinderpopulationen von Jelšovce. Theoretisch ist eine Abnahme der Sterblichkeit von Infans Ia zu Infans Ib und 
wiederum zu Infans II zu erwarten. Wie Tabelle 14 zeigt, weist keine der drei Populationen aus Jelšovce diese Verteilung auf. Basierend auf den Untersuchungen von Schultz, SchmidtSchultz und Kreutz (SCHULTZ et al. 1998) wurden die Kinder der Nitra- und der AunjetitzKultur den Altersklassen neu zugeordnet. Bei allen drei Kulturen werden wider Erwarten mehr Kinder der Gruppe Infans II als der Gruppe Infans I zugeordnet (Tab. 14, Abb. 18 - 20). Der Anteil der Kleinkinder (Infans Ia) ist besonders in Aunjetitz- und Nitra-Kultur mit 5,0 bzw. 8,1 \% sehr niedrig (Abb. 18 - 20). Eher der Erwartung entspricht die Altersverteilung in İkiztepe. Hier werden 57,4 \% der Kinder der Altersklasse I und nur 32,4 \% der Altersklasse II zugeordnet. Fetale Skelete sind aber auch hier mit nur 6,6 \% unterrepräsentiert (Tab. 14).

Tab. 14 Altersverteilung der Kinderpopulationen von Jelšovce und İkiztepe (in Prozent)

\begin{tabular}{|c|c|c|c|c|}
\hline Altersklasse & Mad'arovce & Aunjetitz & Nitra & $\begin{array}{l}\text { İkiztepe } \\
\text { (SCHULTZ 2001a) }\end{array}$ \\
\hline Fetus & $\mathbf{0}$ & $\begin{array}{r}8,3 \\
(\mathrm{n}=4)\end{array}$ & $\mathbf{0}$ & $\begin{array}{r}6,6 \\
(n=9,3)\end{array}$ \\
\hline Infans I & $\begin{array}{r}49,4 \\
(n=39)\end{array}$ & $\begin{array}{r}41,5 \\
(n=19,9)\end{array}$ & $\begin{array}{r}38,3 \\
(n=22,3)\end{array}$ & $\begin{array}{r}57,4 \\
(\mathrm{n}=80,9)\end{array}$ \\
\hline Infans Ia & $\begin{array}{r}14,5 \\
(n=11,5)\end{array}$ & $\begin{array}{r}5,0 \\
(n=2,4)\end{array}$ & $\begin{array}{r}8,1 \\
(n=4,7)\end{array}$ & $\begin{array}{r}25,0 \\
(\mathrm{n}=35,2)\end{array}$ \\
\hline Infans Ib & $\begin{array}{r}34,8 \\
(n=27,5)\end{array}$ & $\begin{array}{r}36,5 \\
(\mathrm{n}=17,5)\end{array}$ & $\begin{array}{r}30,2 \\
(\mathrm{n}=17,5)\end{array}$ & $\begin{array}{r}32,4 \\
(\mathrm{n}=45,7)\end{array}$ \\
\hline Infans II & $\begin{array}{r}50,6 \\
(n=40)\end{array}$ & $\begin{array}{r}43,9 \\
(n=21,1) \\
\end{array}$ & $\begin{array}{r}55,3 \\
(\mathrm{n}=32,1) \\
\end{array}$ & $\begin{array}{r}32,4 \\
(n=45,7)\end{array}$ \\
\hline Juvenis & & $\begin{array}{r}6,3 \\
(\mathrm{n}=3)\end{array}$ & $\begin{array}{r}6,4 \\
(n=3,7)\end{array}$ & $\begin{array}{r}3,6 \\
(n=5,1)\end{array}$ \\
\hline & $\begin{array}{r}100 \\
(n=79)\end{array}$ & $\begin{array}{r}100 \\
(n=48)\end{array}$ & $\begin{array}{r}100 \\
(n=58)\end{array}$ & $\begin{array}{r}100 \\
(n=141)\end{array}$ \\
\hline
\end{tabular}

Skelete von Feten fehlen in der Mad'arovce-Kultur vollständig. Da es äußerst unwahrscheinlich ist, dass in Jelšovce keine Fehl- und Frühgeburten auftraten und keine Neugeborenen starben, ist das Fehlen fetaler und neugeborener Skelete, sowie der relativ niedrige Anteil an Kleinkindern, als artifiziell anzusehen. Mögliche Ursachen sind neben der schnelleren Vergänglichkeit und dem schlechteren Erhalt dünnerer Knochen, auch die Tiefe der Grabgruben. 
Bei kleinen Kindern wurden die Grabgruben in der Regel weniger tief angelegt (vgl. KREUTZ 1997), und sind dadurch stärker von natürlicher Bodenerosion sowie, speziell in Jelšovce, von ackerbaulicher Nutzung des Geländes betroffen.

Ebenfalls denkbar sind Sonderbestattungen Neu- und Frühgeborener (vgl. SCHWIDETZKY 1965). Aus der Übergangsphase von Aunjetitz- zu Mad’arovce-Kultur sind Kinderbestattungen in Pithosgefäßen in den Siedlungen bekannt (FURMÁNEK et al. 1999). V. Podborský beschreibt Kinderbestattungen in Pithosgefäßen im Kerngebiet der, zum gleichen Kulturkomplex wie die Mad’arovce-Kultur gehörenden, Věteřov-Kultur (PODBORSKÝ 1993). Aus dem ägäischen Raum (minoisches Kreta, frühbronzezeitliches Anatolien) sind ebenfalls Kinderbestattungen in Siedlungen in Gefäßen bekannt (SCHACHERMEYR 1964, WITTWER-BACKOFEN 1997). Zurzeit gibt es aus Jelšovce allerdings keine Hinweise auf derartige Bestattungen. In der zugehörige mad'arovcezeitliche Siedlung in Jelšovce wurden keine Kinderskelete gefunden, möglicherweise weil diese nur unvollständig ergraben wurde (KUDLÁČEK 1958, BÁTORA 1986). Die Vergleichbarkeit der Daten aus Jelšovce ist trotz des Kleinkinderdefizits gegeben, da für die drei Populationen vergleichbare Erhaltungsbedingungen vorliegen. Von ähnlichen Bedingungen kann auch im Traisental ausgegangen werden. In den oben genannten Kulturen fehlen somit etwa gleiche Anteile der Kleinkinder, so dass die im Folgenden aufgeführten Daten als vergleichbar gelten können. Eine Hinzuschätzung der fehlenden Kleinkinder ist zum gegenwärtigen Zeitpunkt nicht möglich, da die Untersuchung der erwachsenen Skelete noch nicht abgeschlossen ist und somit die zur Berechnung notwendigen Daten nicht zur Verfügung stehen (BERNER 1997, BOCQUET-APPEL \& MASSET 1982).

\section{IV.2.2. Kindersterblichkeit}

Der Anteil subadulter Individuen in Jelšovce beträgt in der Mad'arovce-Kultur 23,3 \% (n = 79/ 339), in der Aunjetitz-Kultur 36,6 \% und in der Nitra-Kultur 42,0 \%. Andere bronzezeitliche und mittelalterliche Populationen weisen vergleichbare Ergebnisse auf. In Hainburg be- 
trägt die Kindersterblichkeit 30 \%, in Pitten 46 \% (beide Bronzezeit). In Straubing beträgt sie 23,4 \%, bei weiteren mittelalterliche Populationen liegt die Kindersterblichkeit zwischen 23 und 40 \% (KREUTZ 1997, SCHULTZ 1987), andere Untersuchungen ergeben sogar eine Kindersterblichkeit von 45 bis 60 \% (SCHULTZ 1989 b, ARNOLD 1989).

In Jelšovce zeigt sich ein Rückgang der Kindersterblichkeit im Verlauf der frühen Bronzezeit. Der Vergleich der drei Kulturen zeigt bei den Kindern der Aunjetitz-Kultur vorgeburtlich die höchste Mortalität. Allerdings fehlen die Skelete von Säuglingen in Mad'arovce- und NitraKultur vollständig (s. IV.2.1, Tab. 14). Die Absterbekurve (Abb. 21) zeigt, dass die Mortalität nach der Geburt bei den Mad'arovce-Kinder höher als in den beiden anderen Kulturen. Vor allem Säugling und Kleinkinder der Mad'arovce-Kultur hatten schlechtere Lebensbedingungen als gleichaltrige Kinder der beiden anderen Kulturen. Diesen Unterschied in den ersten Lebensjahren zeigen die Werte der Mortalität in der Gruppe Infans Ia. Mit 14,6 \% für Mad'arovce-Kultur starben in dieser Gruppe deutlich mehr Kinder als in der Nitra- und Aunjetitz-Kultur (8,1 bzw. 5,0 \%) (Abb. 18 - 20, Tab. 14).

Bis etwa zum 9. Lebensjahr weisen die Kinder der Nitra-Kultur die niedrigste Mortalitätsrate auf. Im Vergleich der Absterbekurven verläuft die Kurve der Nitra-Kultur deutlich oberhalb derjenigen der anderen beiden Kulturen. Danach gleichen sich alle Kurven einander an und verlaufen in etwa parallel zueinander. Das bedeutet, dass der Rückgang der Kindersterblichkeit im Verlauf der frühen Bronzezeit in Jelšovce mit einer Verschiebung der Sterbehäufigkeit in jüngere Altersklassen einhergeht. In den ersten zwei Lebensjahren verstarben der Mad'arovce-Kultur anteilig mehr Kinder als in Aunjetitz- und Nitra-Kultur.

Bei einem Vergleich der Mortalitätsraten von Frühbronzezeit (Jelšovce) und Mittelalter zeigt sich in den ersten drei Lebensjahren für die mittelalterlichen Populationen eine zum Teil deutlich höhere Mortalität. Besonders in Bettingen ist der Anteil der ganz kleinen Kinder sehr hoch (Tab. 15). Die Absterbekurve von Bettingen flacht als Zeichen des Rückgangs der Sterblichkeit nach dem 4. Lebensjahr ab (Abb. 22 und 23) (vgl. TEMPLIN 1993). Die Kinder von 
Boğazkale weisen bis zum 8. Lebensjahr die steilste Kurve auf (Abb. 22). Hier war die Sterblichkeit nicht nur bei den Kleinkindern wie in Bettingen sondern auch unter den älteren Kindern relativ hoch. Auch in Straubing ist die Sterblichkeit der Kinder unter sechs Jahren größer als in den frühbronzezeitliche Populationen aus Jelšovce. Erst jenseits des 6. Lebensjahres kreuzen sich die Kurven. Nach dem 6. Lebensjahr verlaufen die Kurven von Bettingen und Straubing am flachsten, das heißt, dass die Sterblichkeit bei diesen geringer ist als bei den verglichenen Populationen. Der Vergleich zeigt, dass vor allem die Kinder der Altersklasse Infans I in der Frühbronzezeit bessere Überlebenschancen hatten als gleichaltrige Kinder im Mittelalter. Verglichen mit den Lebensbedingungen der mittelalterlichen Populationen waren diejenigen der Kinder aller drei Kulturen in Jelšovce folglich relativ gut.

Abb. 18 Altersverteilung der Kinder in der Mad'arovce-Kultur von Jelšovce

\section{Altersverteilung in der Kinderpopulation der Mad'arovce- Kultur in Prozent}

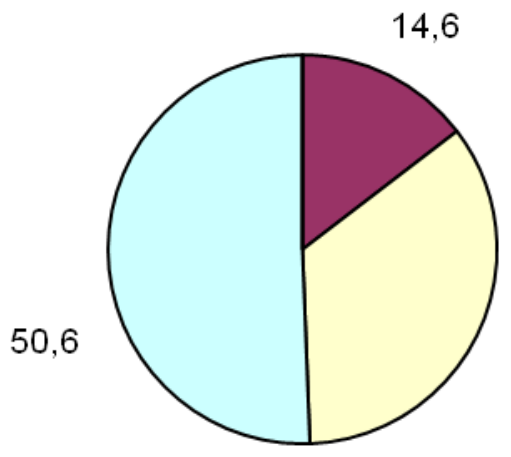

$\operatorname{lnfansla}(\mathrm{n}=11,5 / 79)$

口Infans lb $(\mathrm{n}=27,5 / 79)$

口Infans II $(n=40 / 79)$

34,8 
Abb. 19 Altersverteilung der Kinder in der Aunjetitz-Kultur von Jelšovce

\section{Altersverteilung in der Kinderpopulation der Aunjetitz- Kultur in Prozent}

aFetus $(n=4 / 48)$

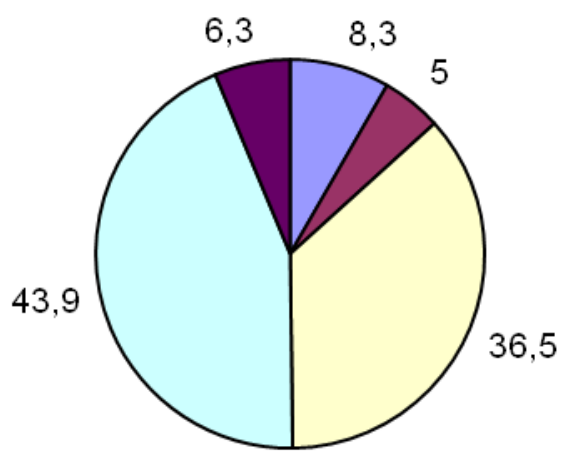

unfans la $(\mathrm{n}=2,4 / 48)$

םlnfans lb $(n=17,5 / 48)$

口Infans II $(n=21,1 / 48)$

Juvenis $(n=3 / 48)$

Abb. 20 Altersverteilung der Kinder in der Nitra-Kultur von Jelšovce

Altersverteilung in der Kinderpopulation der Nitra-Kultur in Prozent

ulnfans la $(n=4,7 / 58)$

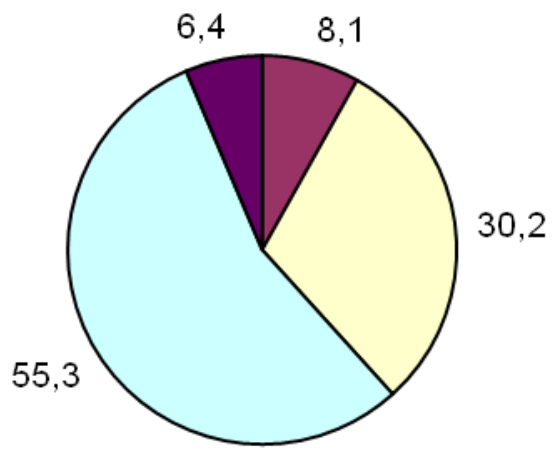

$\operatorname{lnfans~lb}(\mathrm{n}=17,5 / 58)$

口Infans II ( $\mathrm{n}=32,1 / 58)$

aJuvenis ( $\mathrm{n}=3,7 / 58)$ 
Abb. 21 Vergleich der Absterbekurven im Kindesalter von Nitra-, Aunjetitz- und Mad'arovce-Kultur von Jelšovce (BÁTORA \& SCHULTZ 2001)

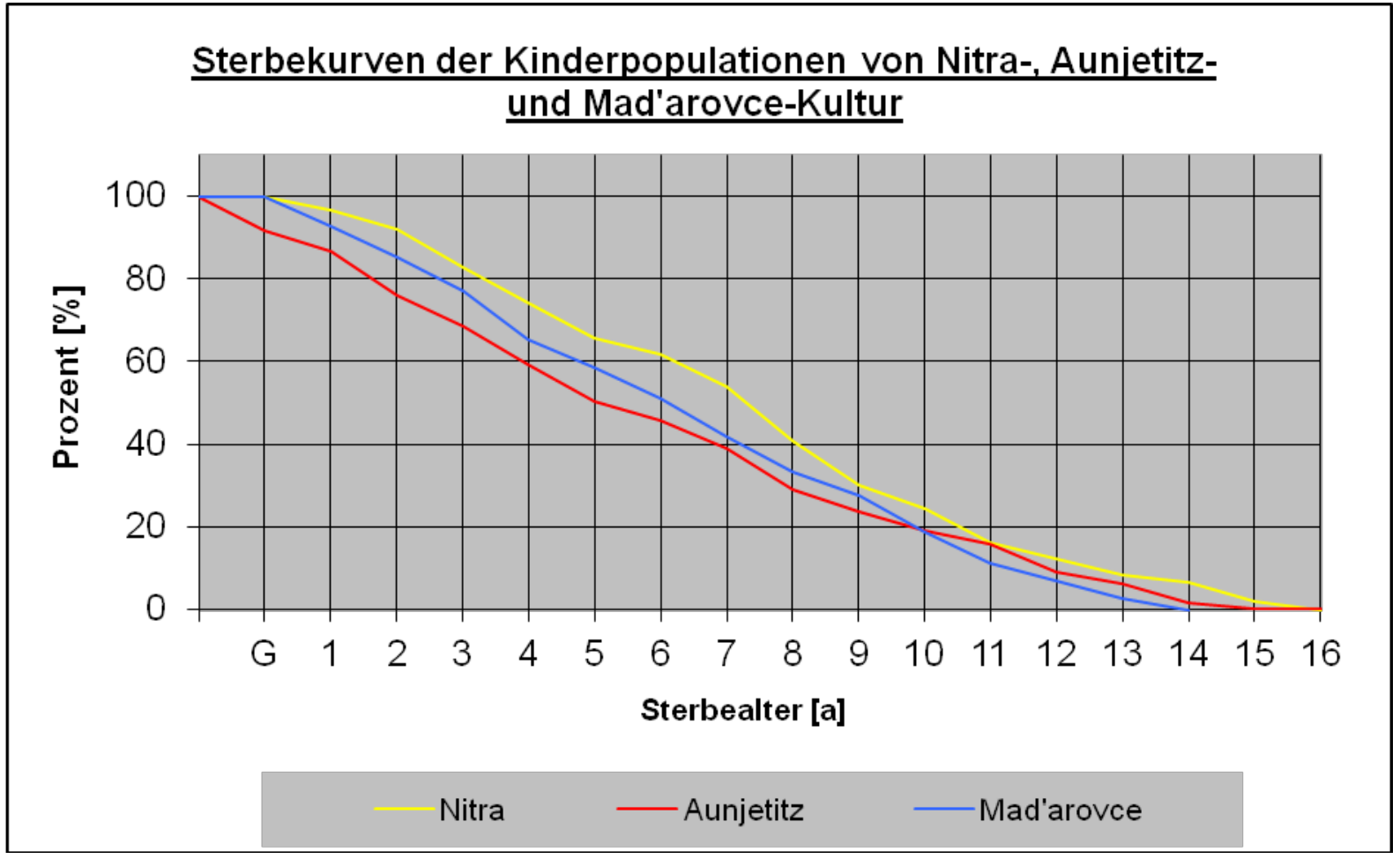

(G: Geburt)

Abb. 22 Vergleich der Absterbekurven im Kindesalter verschiedener bronzezeitlicher und mittelalterlicher Kulturen (BÁTORA \& SCHULTZ 2001, TEMPLIN 1993, KREUTZ 1997, SCHULTZ 1989 b)

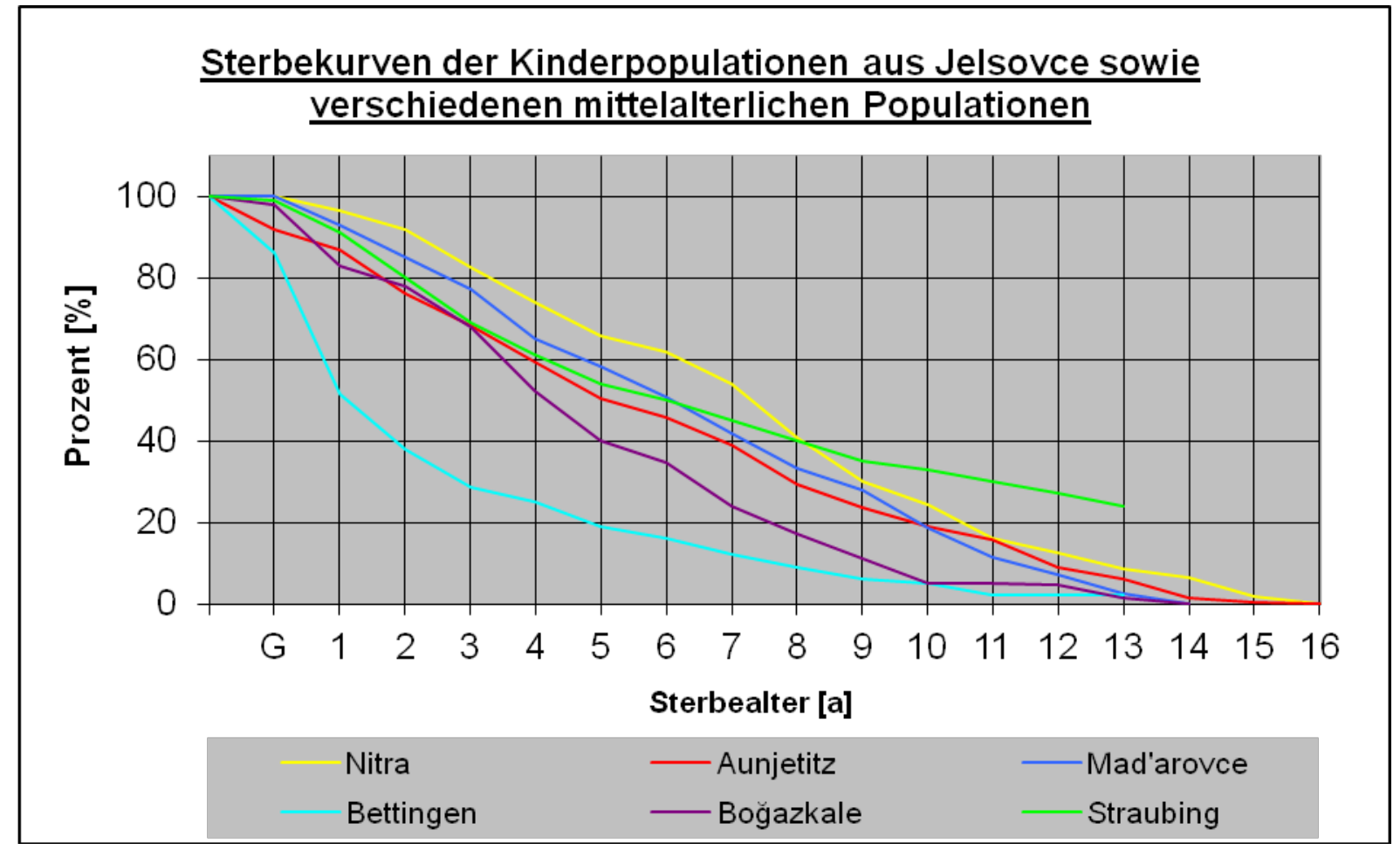

(G: Geburt) 
Tab. 15 Vergleich der differenzierten Mortalität der Populationen von Jelšovce mit mittelalterlichen Populationen

\begin{tabular}{|c|c|c|c|c|c|c|}
\hline & \multicolumn{6}{|c|}{ Population und Autor } \\
\hline & $\begin{array}{l}\text { Jelšovce- } \\
\text { Mad'arovce }\end{array}$ & $\begin{array}{l}\text { Jelšovce- } \\
\text { Aunjetitz }\end{array}$ & $\begin{array}{l}\text { Jelšovce- } \\
\text { Nitra }\end{array}$ & \begin{tabular}{|l} 
Bettingen \\
(Templin \\
1993)
\end{tabular} & $\begin{array}{l}\text { Boğazkale } \\
\text { (Schultz } \\
1989 \text { b) }\end{array}$ & $\begin{array}{l}\text { Straubing } \\
\text { (Kreutz } \\
\text { 1997) }\end{array}$ \\
\hline Alter [Jahre] & $1(\mathrm{x})[\%]$ & $1(\mathrm{x})[\%]$ & $1(\mathrm{x})[\%]$ & $1(\mathrm{x})[\%]$ & $1(\mathrm{x})[\%]$ & $1(\mathrm{x})[\%]$ \\
\hline 7. Fet.mon. & 100 & 100 & 100 & 100 & 100 & 100 \\
\hline Geburt & 100 & 91,7 & 100 & 86 & 98 & 99 \\
\hline 1 & 92,9 & 86,7 & 96,6 & 51,5 & 83 & 91 \\
\hline 2 & 85,2 & 76,0 & 92,0 & 38 & 78 & 80 \\
\hline 3 & 77,2 & 68,4 & 82,6 & 28,5 & 68 & 69 \\
\hline 4 & 65,2 & 59,3 & 74,1 & 25 & 52 & 61 \\
\hline 5 & 58,4 & 50,2 & 65,8 & 19 & 40 & 54 \\
\hline 6 & 50,9 & 45,6 & 61,8 & 16 & 34,5 & 50 \\
\hline 7 & 41,7 & 39,0 & 54,0 & 12 & 24 & 45 \\
\hline 8 & 33,4 & 29,2 & 40,7 & 9 & 17 & 40 \\
\hline 9 & 27,7 & 23,8 & 30,0 & 6 & 11 & 35 \\
\hline 10 & 18,6 & 19,1 & 24,4 & 5 & 5 & 33 \\
\hline 11 & 11,3 & 15,8 & 16,1 & 2 & 5 & 30 \\
\hline 12 & 7,0 & 9,0 & 12,4 & 2 & 4,5 & 27 \\
\hline 13 & 2,6 & 6,2 & 8,4 & 2 & 1,5 & 24 \\
\hline 14 & 0,0 & 1,5 & 6,5 & & 0 & \\
\hline 15 & & 0,3 & 2,0 & & & \\
\hline 16 & & 0,1 & 0,0 & & & \\
\hline
\end{tabular}

(Fet.mon.: Fetalmonat) 
Abb. 23 Vergleich der Absterbekurven im Alter von Geburt bis zum 4. Lebensjahr bei verschiedenen bronzezeitlichen und mittelalterlichen Kulturen (BÁTORA \& SCHULTZ 2001, TEMPLIN 1993, KREUTZ 1997, SCHULTZ 1989 b)

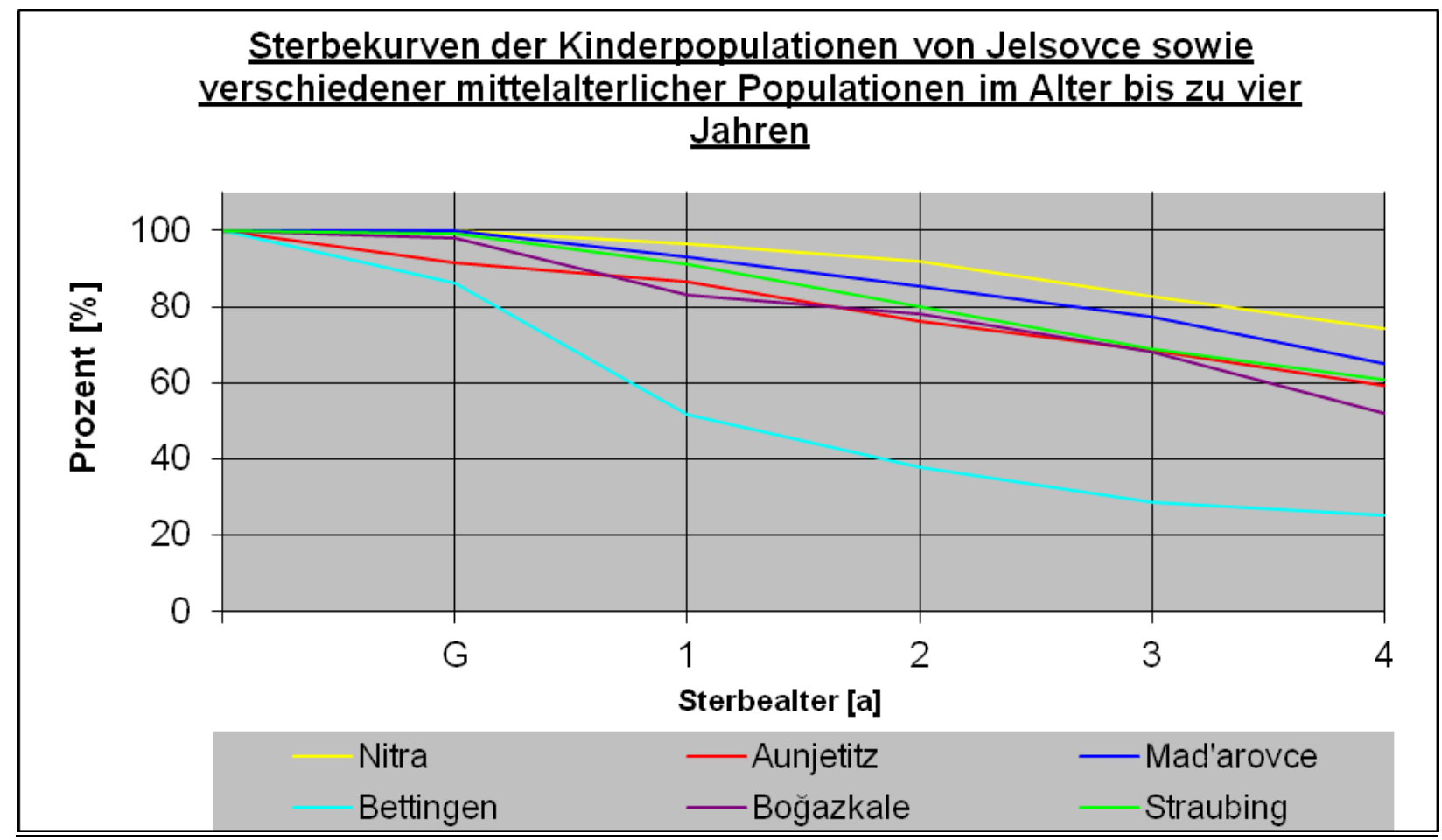

IV.2.3. Vergleich der Kinder- und Säuglingssterblichkeit in der Bronzezeit mit rezenten Daten

Der Vergleich der Kinder- und Säuglingssterblichkeit zwischen der Bronzezeit und der heutigen Zeit ist aufgrund des oben behandelten Kleinkinderdefizits gewissen Schwierigkeiten unterworfen. Außerdem sind die betrachteten Alterszeiträume nicht identisch. Bei den rezenten Daten bezieht sich die angegebene Kindersterblichkeit um den Zeitraum zwischen Geburt und 5. Lebensjahr. Die zum Vergleich verwendeten Daten aus der Bronzezeit umfassen den Zeitraum von Geburt bis zum 6. Lebensjahr. Trotz dieser Ungenauigkeiten soll ein Vergleich bzw. eine Gegenüberstellung von bronzezeitlichen und rezenten Daten erfolgen.

Die rezenten Daten zeigen, dass Kinder- (und Säuglingssterblichkeit) in den reichen Industrienationen kein großes Problem sind (Tab. 17). Die Kindersterblichkeit beträgt weniger als $1 \%$. Anders sieht es in Länder mit niedrigem wirtschaftlichem und medizinischem Entwick- 
lungsstand, den so genannten Entwicklungsländern oder Schwellenländern, aus. Vor allem von (Bürger-) Kriegen und Zerstörung betroffene Länder weisen eine erschreckend hohe Kinderund Säuglingssterblichkeit auf (Sierra Leone, Angola, Afghanistan, Kongo). Kindersterblichkeiten von bis zu 30\% und Säuglingssterblichkeiten zwischen 15 und 20 \% werden angegeben (Tab. 17). Vergleichbare Zahlen ergeben sich für die frühbronzezeitliche Kindersterblichkeit in Nitra- und Traisental. Die Mortalität liegt im Alter von der Geburt bis zum sechsten Lebensjahr zwischen 11 und 17 \% (Tab. 16). Wobei aufgrund oben genannter Unterschiede das Ergebnis nur als grobe Richtungsweisung gewertet werden kann. In Kriegsgebieten sind Infektionserkrankungen begünstigt durch Mangelernährung, unzureichende Hygiene und fehlende medizinische Versorgung die wichtigsten Ursachen für den Tod von Kindern und Säuglingen. Auch in der Bronzezeit waren Mangelernährung und Infektionserkrankungen einhergehend mit mangelhafter Hygiene ein Problem für Kinder und Erwachsenen. Erst in den letzten 100 Jahren war der Rückgang der Kindersterblichkeit in Teilen der Welt aufgrund verbesserter hygienischer Bedingungen und Verbesserung des Lebensstandards größerer Bevölkerungsschichten erheblich.

Tab. 16 Mortalität von der Geburt bis zum sechsten Lebensjahr in verschiedenen bronzezeitlichen Kulturen (BÁTORA \& SCHUlTZ 2001, SCHULTZ 1993, TESCHLER-NIKOLA 1994)

\begin{tabular}{|l|l|}
\hline Kultur & $\begin{array}{l}\text { Sterblichkeit in Prozent } \\
{[\%]}\end{array}$ \\
\hline Jelšovce-Mad'arovce & $11,8(40 / 339)$ \\
\hline Franzhausen-I & 15,7 \\
\hline Gemeinlebarn-F & 16,3 \\
\hline Pottenbrunn & 16,4 \\
\hline Jelšovce-Nitra & 16,6 \\
\hline Jelšovce-Aunjetitz & 16,7 \\
\hline
\end{tabular}


Tab. 17 Säuglings- und Kindersterblichkeit 2000 weltweit (ALBRECHT et al. 2002)

\begin{tabular}{|l|r|l|}
\hline Land & $\begin{array}{l}\text { Kindersterblichkeit } \\
\text { in \% } \\
\text { (Geburt bis zum 5.Lj) }\end{array}$ & $\begin{array}{l}\text { Säuglingssterblichkeit } \\
\text { in \% } \\
\text { (innerhalb des 1.Lj) }\end{array}$ \\
\hline Österreich & 0,5 & \\
\hline Deutschland & 0,5 & \\
\hline USA & 0,8 & \\
\hline Slowakei & 0,9 & \\
\hline Argentinien & 2,1 & \\
\hline Albanien & 3,1 & \\
\hline Philippinen & 4 & \\
\hline Ägypten & 4,3 & \\
\hline Türkei & 4,5 & \\
\hline Peru & 5 & \\
\hline Bolivien & 8 & \\
\hline Indien & 9,6 & \\
\hline Botsuana & 10,1 & \\
\hline Simbabwe & 11,7 & \\
\hline Nigeria & 18,4 & \\
\hline Ruanda & 18,7 & \\
\hline Kongo & 20,7 & \\
\hline Afghanistan & 25,7 & \\
\hline Angola & 29,5 & \\
\hline Sierra Leone & 31,6 & \\
\hline (Lj:: Lebensjatr & & \\
\hline
\end{tabular}

(Lj.: Lebensjahr)

\section{IV.3. Zahnerkrankungen}

Zahnerkrankungen zeigen in ihrer Verteilung in den drei Populationen aus Jelšovce deutliche Unterschiede. Von Zahnstein und Parodontopathien sind die Kinder der Mad'arovce-Kultur mit Abstand am häufigsten (55,1 bzw. 63,4 \%), die Kinder der Aunjetitz-Kultur mit 32 bzw. 45,8 \% auch noch relativ stark betroffen. Im Gegensatz dazu ist das Ausmaß des Befalls in der Nitra-Kultur mit 2,6 bzw. 25,6 \% relativ gering. Karies tritt am häufigsten bei den Kindern der Aunjetitz-Kultur ( $\mathrm{n}=$ 5/ 25: $20 \%$ ) auf. In Nitra-Kultur und Mad'arovce-Kultur ist sie mit 7,7 bzw. 6,1 \% (n=3/ 39 bzw. 3/ 49) deutlich seltener (Tab. 18). 
Tab. 18 Das Auftreten von Zahnstein, Parodontopathien und Karies in den frühbronzezeitlichen Populationen von Jelšovce.

\begin{tabular}{|l|lr|ll|ll|}
\hline & \multicolumn{2}{|l|}{$\begin{array}{l}\text { Zahnstein } \\
\text { in [\%] }\end{array}$} & $\begin{array}{l}\text { Parodontopathien } \\
\text { in [\%] }\end{array}$ & $\begin{array}{l}\text { Karies } \\
\text { in [\%] }\end{array}$ \\
\hline Mad'arovce & $55,1 \quad(27 / 49)$ & $63,4 \quad(26 / 41)$ & 6,1 & $(3 / 49)$ \\
\hline Aunjetitz (SCHULTZ 2001 a) & $32 \quad(8 / 25)$ & $45,8 \quad(11 / 24)$ & 20 & $(5 / 25)$ \\
\hline Nitra (SCHULTZ et al. 1998) & $2,6 \quad(1 / 39)$ & 25,6 & 7,7 & $(3 / 39)$ \\
\hline
\end{tabular}

Im Vergleich mit weiteren Populationen fällt auf, dass die bronzezeitlichen Kinder deutlich häufiger als die mittelalterlichen Kinder von Parodontopathien befallen sind. 70 bis $80 \%$ Erkrankte in den mittelalterlichen und neolithischen Populationen stehen 15 bis 45 \% in den bronzezeitlichen Populationen gegenüber (Tab. 20). Eine Ausnahme stellen die Populationen der späten Frühbronzezeit, Jelšovce-Mad'arovce und Hainburg dar. Sie erreichen mit einer Erkrankungsrate von 64 bzw. 76 \% etwa das Niveau der mittelalterlichen und neolithischen Populationen (Tab. 20). Hiermit zeigt sich, einer Zunahme der Erkrankungsrate im Verlauf der Frühbronzezeit.

Ähnliches gilt in Bezug auf die Häufigkeiten von Karies. Die Kinder aus Mittelalter und Neolithikum sind tendenziell häufiger betroffen als die der Bronzezeit. Jelšovce-Aunjetitz bildet mit 20\% eine Ausnahme (Tab. 19). Für Zahnstein liegen leider von den bronzezeitlichen Populationen aus Niederösterreich ebenso wie von den hier zitierten mittelalterlichen Populationen keine Vergleichsdaten vor.

Da sowohl Karies und Zahnstein als auch Parodontopathien gehäuft bei unzureichender Mundhygiene auftreten, ist es sinnvoll diese Erkrankungen im Zusammenhang zu betrachten, obwohl hinsichtlich Zahnpflege und Mundhygiene in den prähistorischen Populationen nicht von einer systematischen Pflege ausgegangen werden kann (SCHULTZ 2001 a, SCHULTZ et al. 1998). Zahnstein und Parodontopathien treten in Jelšovce in der Mad'arovce-Kultur am häu- 
figsten auf, gefolgt von der Aunjetitz-Kultur. In der Nitra-Kultur sind sie am seltensten (Tab. 18).

Niedrige Häufigkeiten bei Parodontopathien und Karies liegen in Franzhausen-I und İkiztepe vor. Die Kinder aus Wandersleben, Bettingen, Straubing und Hainburg sind im Gegensatz dazu von beiden Erkrankungen relativ stark betroffen. Eine Ausnahme stellt JelšovceAunjetitz dar. Bei hoher Kariesfrequenz tritt nur eine mäßige Erkrankungsrate mit Parodontopathien auf.

Im Zusammenhang mit Parodontopathien ist auch ein Vitamin C Mangel wichtig (s. IV.7). In allen Populationen treten Parodontopathien häufiger auf als ein Skorbut (Tab. 20). Außerdem zeigt sich, dass mit Abnahme der Häufigkeit von Parodontopathien auch die Häufigkeit von Skorbut abnimmt. Dieser Unterschied tritt auf, da ein Skorbut zwar eine wichtige aber nicht die einzige Ursache für Parodontopathien ist. Mangelnde Mundhygiene, Infektionen oder Immunschwäche können diese ebenfalls auslösen.

Die Kinder aus Straubing sind von beiden Erkrankungen am häufigsten betroffen, die Kinder aus Franzhausen-I am wenigsten (Tab. 20). Insgesamt sind Parodontopathien in Mittelalter und Neolithikum häufiger als in der Frühbronzezeit. Gegen Ende der Frühbronzezeit sind sie wiederum häufiger als zu Beginn der Frühbronzezeit. 
Tab. 19 Die Häufigkeit des Auftretens von Karies in verschiedenen bronzezeitlichen, neolithischen und mittelalterlichen Kinderpopulationen (Kariesfrequenz = Verhältnis von gesunden zu kranken Gebissen). (CARLI-THIELE 1996, KREUTZ 1997, SCHULTZ et al. 1998, SCHULTZ 2001 a, TEMPLIN 1993)

\begin{tabular}{|l|ll|}
\hline Kultur & $\begin{array}{l}\text { Häufigkeit in Prozent } \\
{[\%]}\end{array}$ \\
\hline Bettingen & 31,2 \\
\hline Jelšovce-Aunjetitz & 20 & $(5 / 25)$ \\
\hline Wandersleben & 14,3 & $(5 / 32)$ \\
\hline Straubing & 14,3 & $(24 / 168)$ \\
\hline Hainburg & 13,2 & $(5 / 38)$ \\
\hline Gemeinlebarn-F & 11,9 & $(5 / 42)$ \\
\hline Jelšovce-Nitra & 7,7 & $(3 / 39)$ \\
\hline Jelšovce-Mad'arovce & 6,1 & $(3 / 49)$ \\
\hline Franzhausen-I & 4,4 & $(4 / 91)$ \\
\hline İkiztepe & 2,3 & $(2 / 91)$ \\
\hline
\end{tabular}

Tab. 20 Die Häufigkeit des Auftretens von Parodontopathien und Skorbut in verschiedenen bronzezeitlichen, neolithischen und mittelalterlichen Kinderpopulationen. (CARLIThiele 1996, KREUTZ 1997, SCHUltZ et al. 1998, SCHUltZ 2001 a, TEMPLIN 1993)

\begin{tabular}{|c|c|c|}
\hline Kultur & $\begin{array}{l}\text { Parodontopathien } \\
\text { Häufigkeit in Prozent } \\
{[\%]}\end{array}$ & $\begin{array}{l}\text { Skorbut } \\
\text { Häufigkeit in Prozent } \\
{[\%]}\end{array}$ \\
\hline Straubing & $(108 / 137)$ & (57/ 159) \\
\hline Hainburg & $(28 / 37)$ & $(0 / 32)$ \\
\hline Bettingen & 70,4 & \\
\hline Wandersleben & $(38 / 57)$ & $(22 / 68)$ \\
\hline Jelšovce-Mad'arovce & $(26 / 41)$ & $(12 / 49)$ \\
\hline Jelšovce-Aunjetitz & $(11 / 24)$ & $(10 / 43)$ \\
\hline Jelšovce-Nitra & 25,6 & $(6 / 47)$ \\
\hline İkiztepe & $(16 / 88)$ & $(17 / 123)$ \\
\hline Franzhausen-I & $(7 / 45)$ & $(7 / 110)$ \\
\hline
\end{tabular}




\section{$\underline{\text { IV.4. Entzündungserkrankungen }}$}

Vergleicht man die Häufigkeiten des Auftretens von Entzündungserkrankungen in den drei Populationen von Jelšovce so fällt auf, dass die Kinder der Mad'arovce-Kultur häufiger von entzündlichen Erkrankungen der Nasennebenhöhlen und Ohren sowie des Endocranialraums betroffen sind, als die Kinder der anderen Kulturen (Tab. 21, Abb. 24). Die Kinder der NitraKultur sind insgesamt am seltensten betroffen.

Bei den Kindern der Mad'arovce-Kultur tritt eine Sinusitis maxillaris mit 35,5 \% (n = 6/ 17) auf, bei den Kindern der Aunjetitz-Kultur sind es nur 15,8 \% (n = 3/ 19). Die Kinder der NitraKultur sind überhaupt nicht betroffen $(n=0 / 43)$. Auch eine Otitis media tritt bei keinem Kind der Nitra-Kultur auf $(n=0 / 43)$. In der Aunjetitz-Kultur sind 8\% $(n=2 / 24)$, bei der Mad'arovce-Kultur sogar 12,5 \% $(n=3 / 24)$ der Kinder betroffen. Die Perisinusitis zeigt mit 26,2 \% ( $=11 / 42)$ erkrankter Kinder in der Mad'arovce-Kultur, 17,2 \% $(n=5 / 31)$ in der Aunjetitz-Kultur und 7,0 \% in der Nitra-Kultur die gleiche Verteilung.

Meningeale Reizungen treten in der Mad'arovce-Kultur bei 26,7 \% ( $\mathrm{n}=12 / 45)$, in der Aunjetitz-Kultur bei 20,7 \% $(n=6 / 29)$ und in der Nitra-Kultur bei 11,6 \% $(n=5 / 43)$ der Kinder auf. Hirndrucksymptomatik lässt sich in der Mad'arovce-Kultur bei 34,8 \% ( $n=16$ / 46), in der Aunjetitz-Kultur bei 9,7 \% $(n=3 / 31)$ und in der Nitra-Kultur bei 2,3 \% $(n=1 / 43)$ der Kinder beobachten. Bei allen oben aufgeführten Erkrankungen sind jeweils die Kinder der Mad'arovce-Kultur am häufigsten betroffen, die der Nitra-Kultur am seltensten. Die einzige Ausnahme bei dieser Häufigkeitsverteilung bildet die Mastoiditis (Tab. 21, Abb. 24). Sie tritt bei den Kindern der Mad'arovce-Kultur kaum auf. Lediglich ein Kind ist betroffen $(n=1 / 33$ : 3 \%), obwohl die Otitis media als Ursache für eine Mastoiditis mit 12,5 \% ( $=3$ / 24) relativ häufig ist. Vermutlich sind die Kinder mit Otitis media gestorben, bevor sich eine Mastoiditis entwickeln konnte. Anders verhält es bei den Kindern der Aunjetitz-Kultur und Nitra-Kultur. Hier ist die Mastoiditis mit 12,5 bzw. 11,6 \% (n = 3/ 23 bzw. 5/ 43) etwa gleich häufig. Be- 
trachtet man die endocranialen Erkrankungen isoliert, so zeigt sich für Aunjetitz-Kultur und Nitra-Kultur ein entsprechendes Krankheitsprofil (Abb. 25). Meningeale Reizungen treten bei beiden Populationen am häufigsten auf; die Perisinusitis ist etwas seltener. Nur ein kleiner Teil der Kinder zeigt auch eine Hirndrucksymptomatik. Anders verhält es sich bei den Kindern der Mad'arovce-Kultur. Hier sind meningeale Reizungen und Perisinusitiden etwa gleich häufig. Außerdem weisen relativ viele Kinder eine Hirndrucksymptomatik auf (Abb. 25).

Eine Zusammenstellung der Krankheitshäufigkeiten in anderen Populationen (s. Tab. 24 und Abb. 26) zeigt, dass die Kinder der Mad'arovce-Kultur von Jelšovce auch im Vergleich mit diesen relativ häufig von Entzündungserkrankungen betroffen sind.

Die Sinusitis maxillaris ist in Jelšovce-Mad'arovce mit 35,3 \% $(n=6 / 17)$ auch im Vergleich mit Wandersleben ( $n=7 / 39: 18 \%)$, Franzhausen-I $(n=4 / 52: 7,7 \%)$, İkiztepe $(n=4 / 60: 6,7$ \%) und Hainburg ( $\mathrm{n}=$ 0/ 18: 0 \%) sehr hoch (Tab. 24 a) (s. IV.7).

Von einer Sinusitis frontalis sind zwei von sieben Kindern aus Jelšovce-Mad'arovce betroffen $(n=2 / 7: 28,6 \%)$. Die Kinder aus Wandersleben und İkiztepe sind mit 8,3\% $(n=2 / 24)$ bzw. 6,3 \% ( $\mathrm{n}=1 / \mathrm{16})$ deutlich seltener betroffen (Tab. 24 c). Bei den Kindern aus Franzhausen-I lassen sich keine Erkrankungen der Sinus frontales nachweisen $(\mathrm{n}=0$ / 28).

Eine Otitis media trat in İkiztepe mit 16,3 \% $(\mathrm{n}=8 / 49)$ etwas häufiger auf als in JelšovceMad'arovce mit 12,5 \% ( $\mathrm{n}=3 / 24)$ (Tab. 21, Tab. 24 d). Für die Populationen aus Niederösterreich fehlen leider die Vergleichsdaten. Diese wären interessant, da bei der Zusammenfassung von Otitis media und Pneumatisationshemmungen des Processus mastoideus die Erkrankungshäufigkeiten von Gemeinlebarn-F, Hainburg und Pitten mit 33,3 \% (n = 9/ 27), 28,9 $\%(n=11 / 38)$ und 26,3 \% (n = 5/ 19) zum Teil deutlich über der von Jelšovce-Mad'arovce (n = 9/ 39: 23,1\%) liegen (Tab. 24 b). In İkiztepe liegen sie mit 19,6\% $(n=10 / 51)$ etwas niedriger und in Franzhausen-I mit 10,7 \% $(n=6 / 56)$ noch deutlich darunter. 
Eine Mastoiditis tritt in İkiztepe mit 21,7\% $(n=10 / 46)$ deutlich häufiger auf als bei allen drei Populationen aus Jelšovce, dort sind zwischen 3 und 12,5 \% der Kinder betroffen (n = 1/ 33: $3 \% ; n=5 / 43: 11,6 \% ; n=3 / 24: 12,5 \%)$ (Tab. 21, Tab. 24 f).

Eine Stomatitis wiederum ist mit 35,7 \% $(n=10 / 28)$ in Jelšovce-Mad'arovce häufig. Nur in Wandersleben sind mit 36,7 \% $(n=18 / 49)$ noch mehr Kinder erkrankt. Auch in Aiterhofen ist die Erkrankungsrate mit 33,3 \% $(n=2 / 6)$ betroffener Kinder vergleichbar hoch. In İkiztepe $(n=10 / 64: 15,6 \%)$, Franzhausen-I $(n=6 / 57: 10,5 \%)$ und Hainburg $(n=0 / 26: 0$ \%) ist ein Auftreten deutlich seltener (Tab. 24 e). In diesem Zusammenhang ist die Stomatitis in Beziehung zum Skorbut zu betrachten (vgl. IV.5, AsCHOFF 1919). Die hohe Skorbuthäufigkeit in Jelšovce-Mad'arovce ist vermutlich für das häufige Auftreten einer Stomatitis mit verantwortlich. In allen vier verglichenen Populationen ist eine Stomatitis häufiger als ein Skorbut, da der Skorbut nicht die einzige Ursache für eine Stomatitis ist (vgl. IV.3). In İkiztepe und Franzhausen-I sind es gleichsinnig weniger Fälle als in Jelšovce-Mad'arovce. In Hainburg treten beide Erkrankungen nicht auf (Tab. 22).

Im Vergleich von Hirndrucksymptomatik und meningealen Reizungen zwischen den bronzezeitlichen Populationen sind die Kinder von Jelšovce-Mad'arovce in beiden Kategorien am häufigsten betroffen. Die Häufigkeit liegt bei verschiedenen bronzezeitlichen Kinderpopulationen für meningeale Reizungen zwischen 10 und 27 \% und für Hirndrucksymptomatik zwischen 5 und 34 \% (Tab. 24 g - j). Ein Vergleich mit mittelalterlichen Populationen zeigt, dass meningeale Erkrankungen im Mittelalter deutlich häufiger auftraten als in der Bronzezeit (45 - $65 \%$ versus 10 - 30 \%) (Tab 24 i und j), Hirndrucksymptomatik wurde jedoch bei keinen mittelalterlichen Kind nachgewiesen (0 \% versus 2 - 34 \%) (Abb. 26, Tab. 24 g und h). Perisinusitiden sind in der Bronzezeit mit Ausnahme von Jelšovce-Mad'arovce und -Aunjetitz (26,2 bzw. 17,2 \%, $n=11 / 42$ bzw. 5/ 31) mit 3 bis $10 \%$ eher selten. Jelšovce-Mad'arovce ist mit fast $27 \%$ vergleichbar mit dem mittelalterlichen Barbing (27,3\%). Insgesamt sind die 
mittelalterlich Kinder (13 - 27 \%) häufiger als die bronzezeitlichen Kinder (3 - $10 \%$ ) betroffen (Tab. 24 k).

Neben diesen deutlichen Unterschieden zwischen Bronzezeit und Mittelalter gibt es in der Bronzezeit zwischen den einzelnen Populationen ebenfalls Unterschiede. Bei fast allen bronzezeitlichen Populationen sind meningeale Reizungen deutlich häufiger als Hirndrucksymptomatik (10 - 20 \% versus 2 - $10 \%$ ) (Abb. 26). Perisinusitiden sind in allen Populationen seltener als Meningeale Reizungen. Sehr Ähnliche Profile weisen Jelšovce-Aunjetitz, Jelšovce-Nitra, Pitten und Gemeinlebarn-F auf (Abb. 26). Meningeale Reizungen treten hier häufiger auf als Perisinusitiden, diese wiederum häufiger als Hirndrucksymptomatik. Ebenfalls ähnlich ist die Verteilung in İkiztepe und Franzhausen-I. Dort sind Perisinusitiden seltener als Hirndrucksymptomatik, die meningealen Reizungen treten ebenfalls am häufigsten auf (Abb. 26). Ausnahmen stellen Hainburg und Jelšovce-Mad'arovce dar. Eine Hirndrucksymptomatik tritt mit 17,1 $(n=6 / 35)$ bzw. 34,8 \% $(n=16 / 46)$ bei beiden vergleichsweise häufig auf. In Jelšovce-Mad'arovce ist sie sogar häufiger als meningeale Reizungen (Abb. 26, Tab. 24 g - j).

Die Häufigkeit von Entzündungen des Sinus sagittalis superior und des Sinus transversus rechts sind für Franzhausen-I und Jelšovce-Mad'arovce in etwa vergleichbar (S.sag.sup. $n=2$ / 41: 4,9 \% bzw. $n=1 / 32: 3,1 \%$; S. transv. re. $n=4 / 44: 9,1 \%$ bzw. $n=2 / 23: 8,7 \%$; Tab. 23). Entzündungen der Sinus sigmoidei treten in Jelšovce-Mad'arovce 7- bis 10-mal häufiger auf als in Franzhausen-I. In Jelšovce-Mad'arovce sind die Sinus sigmoidei rechts zu 11,7 \% (n $=2 / 17)$ und links zu 38,9 \% $(n=7 / 18)$ betroffen. In Franzhausen-I sind es rechts 1,6 \% $(n=$ 1/ 61) und links 3,6 \% ( $\mathrm{n}=2 / 55)$. Weiterhin auffällig ist, dass bei beiden Populationen die linken Seiten häufiger betroffen sind als die Rechten (Tab. 23). Meningeale Reaktionen als mögliche Ursachen von Perisinusitiden sind in Jelšovce-Mad'arovce und in Franzhausen-I mit 26,7 \% ( $n=12 / 45)$ bzw. 22,2 \% ( $n=22 / 99)$ in etwa gleich häufig und können daher den Häufigkeitsunterschied nicht hinreichend erklären (Tab. 24 j). Mittelohrentzündungen als 
weitere mögliche Ursache für Perisinusitiden, besonders des Sinus sigmoideus, sind in Jelšovce-Mad'arovce häufiger als in Franzhausen-I (Mittelohrentzündungen und Pneumatisationshemmungen $n=9$ / 39: 23,1 \% versus $n=6$ / 58: 10,7 \%; Tab. 21, Tab. 24 b) und daher als Ursache für den beobachteten Unterschied denkbar.

Zusammenfassend lässt sich sagen, dass Entzündungserkrankungen im Mittelalter insgesamt tendenziell häufiger sind als in Jelšovce-Mad'arovce. In dieser Population treten sie wiederum häufiger auf als in den übrigen frühbronzezeitlichen Populationen aus Niederösterreich und Jelšovce. Auch bei den Mangelerkrankungen nimmt die Erkrankungshäufigkeit im Verlauf der Frühbronzezeit zu (vgl. IV.5).

Tab. 21 Entzündliche Erkrankungen der Nasennebenhöhlen, Mittelohren und des Endocranialraums verschiedener frühbronzezeitlichen Populationen.

(SCHULTZ 2001 a, SCHULTZ et al. 1998)

\begin{tabular}{|c|c|c|c|c|c|c|}
\hline & \multicolumn{6}{|c|}{ Erkrankungshäufigkeit in Prozent [\%] } \\
\hline & \begin{tabular}{|l|} 
Jelšovce- \\
Mad'arovce
\end{tabular} & $\begin{array}{l}\text { Jelšovce- } \\
\text { Aunjetitz }\end{array}$ & \begin{tabular}{|l} 
Jelšovce- \\
Nitra
\end{tabular} & Íkiztepe & \begin{tabular}{|l|} 
Franz- \\
hausen-I
\end{tabular} & Hainburg \\
\hline $\begin{array}{l}\text { Sinusitis } \\
\text { maxillaris }\end{array}$ & $35,3 \quad(6 / 17)$ & $15,8(3 / 19)$ & $0 \quad(0 / 43)$ & $6,7 \quad(4 / 60)$ & $7,7 \quad(4 / 52)$ & $(0 / 18)$ \\
\hline Otitis media & $12,5 \quad(3 / 24)$ & $8 \quad(2 / 24)$ & $0 \quad(0 / 43)$ & $16,3 \quad(8 / 49)$ & & \\
\hline $\begin{array}{l}\text { Otitis media }+ \\
\text { Pneumat.hemm. }\end{array}$ & $23,1 \quad(9 / 39)$ & $16 \quad(4 / 24)$ & & $19,6(10 / 51)$ & $10,7 \quad(6 / 56)$ & $28,9(11 / 38)$ \\
\hline Mastoiditis & $3 \quad(1 / 33)$ & $12,5(3 / 23)$ & $11,6(5 / 43)$ & $21,7(10 / 46)$ & & \\
\hline $\begin{array}{l}\text { Hirndruck- } \\
\text { symptomatik }\end{array}$ & $34,8(16 / 46)$ & $9,7 \quad(3 / 31)$ & $2,3 \quad(1 / 43)$ & $5,1 \quad(6 / 117)$ & $10,1(10 / 99)$ & $17,1 \quad(6 / 35)$ \\
\hline $\begin{array}{l}\text { Meningeale } \\
\text { Reizungen }\end{array}$ & $26,7(12 / 45)$ & $20,7(6 / 29)$ & $11,6(5 / 43)$ & $9,4(11 / 117)$ & $22,2(20 / 99)$ & $17,1 \quad(6 / 35)$ \\
\hline Perisinusitis & $26,2(11 / 42)$ & $17,2(5 / 31)$ & $7,0 \quad(3 / 43)$ & $2,8 \quad(4 / 144)$ & $9,8(8 / 82)$ & $(1 / 36)$ \\
\hline
\end{tabular}

(Pneumat.hemm.: Pneumatisationshemmungen) 
Tab. 22 Die Häufigkeiten von Stomatitis und Skorbut bei verschiedenen bronzezeitlichen Kinderpopulationen (in Prozent). (SCHULTZ 2001 a, SCHULTZ et al. 1998)

\begin{tabular}{|l|lc|lc|l|l|}
\hline Kultur & \multicolumn{2}{|l|}{ Stomatitis in [\%] } & \multicolumn{2}{|l|}{ Skorbut in [\%] } & $\begin{array}{l}\text { Stomatitis und } \\
\text { Skorbut in [\%] }\end{array}$ \\
\hline Jel.-Mad'arovce & 35,7 & $(10 / 28)$ & 24,5 & $(12 / 49)$ & 60 & $(6 / 10)$ \\
\hline İkiztepe & 15,6 & $(10 / 64)$ & 13,8 & $(17 / 123)$ & \\
\hline Franzhausen-I & 10,5 & $(6 / 57)$ & 6,4 & $(7 / 110)$ & \\
\hline Hainburg & 0 & $(0 / 26)$ & 0 & $(0 / 32)$ & \\
\hline
\end{tabular}

Tab. 23 Detaillierte Aufstellung der Erkrankungen der venösen Hirnblutleiter in Jelšovce-Mad'arovce und Franzhausen-I. (SCHULTZ 2001 a)

\begin{tabular}{|ll|ll|lc|}
\hline & \multicolumn{3}{|c|}{ Häufigkeit in Prozent [\%] } \\
\hline Hirnblutleiter & \multicolumn{3}{|c|}{ Franzhausen-I } & Jelšovce-Mad'arovce \\
\hline Sinus sagittalis superior & 4,9 & $(2 / 41)$ & 3,1 & $(1 / 32)$ \\
\hline rechter & Sinus transversus & 9,1 & $(4 / 44)$ & 8,7 & $(2 / 23)$ \\
\hline linker & Sinus transversus & 13,3 & $(6 / 45)$ & 0 & $(0 / 20)$ \\
\hline rechter & Sinus sigmoideus & 1,6 & $(1 / 61)$ & 11,7 & $(2 / 17)$ \\
\hline linker & Sinus sigmoideus & 3,6 & $(2 / 55)$ & 38,9 & $(7 / 18)$ \\
\hline
\end{tabular}


Abb. 24 Entzündliche Erkrankungen der Nasennebenhöhlen, Mittelohren und des

Endocranialraums in den drei frühbronzezeitlichen Kinderpopulationen von Jelšovce

(SCHULTZ et al. 1998)

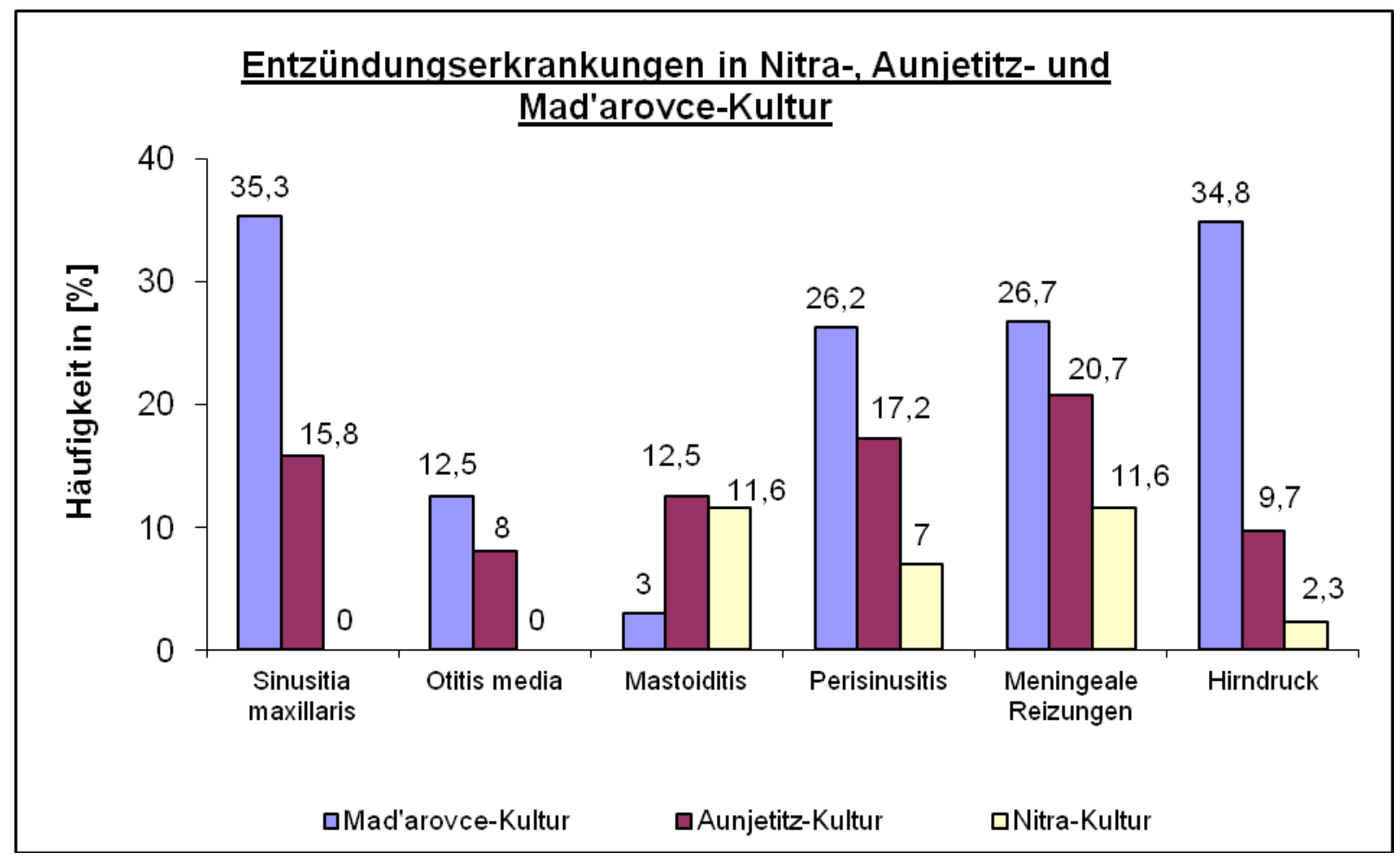

\begin{tabular}{|l|l|l|l|l|l|l|}
\hline & \multicolumn{6}{|c|}{ Zahlen absolut } \\
\hline & Sin. max. & $\begin{array}{l}\text { Otitis } \\
\text { med. }\end{array}$ & Mastoiditis & Perisinusitis & $\begin{array}{l}\text { Mening. } \\
\text { R. }\end{array}$ & Hirndruck \\
\hline Mad'arovce & $6 / 17$ & $3 / 24$ & $1 / 33$ & $11 / 42$ & $12 / 45$ & $16 / 46$ \\
\hline Aunjetitz & $3 / 19$ & $2 / 24$ & $3 / 23$ & $5 / 31$ & $6 / 29$ & $3 / 31$ \\
\hline Nitra & $0 / 43$ & $0 / 43$ & $5 / 43$ & $1 / 43$ & $5 / 43$ & $3 / 43$ \\
\hline
\end{tabular}

(Sin. max.: Sinusitis maxillaris, med.: media, Mening. R.: Meningeale Reizungen) 
Tab. 24 Das Auftreten verschiedener entzündlicher Erkrankungen in verschiedenen bronzezeitlichen, neolithischen und mittelalterlichen Populationen (CARLI-THIELE \& SCHULTZ 2001, SCHULTZ et al. 1998, SCHULTZ 1993, SCHULTZ 2001 a und 2001 b)

a) Sinusitis maxillaris:

\begin{tabular}{lll} 
Jel.-Mad'arovce & $35,3 \%$ & $(6 / 17)$ \\
\hline Wandersleben & $18 \%$ & $(7 / 39)$ \\
Jel.-Aunjetitz & $15,8 \%$ & $(3 / 19)$ \\
Franzhausen-I & $7,7 \%$ & $(4 / 52)$ \\
İkiztepe & $6,7 \%$ & $(4 / 60)$ \\
Hainburg & $0 \%$ & $(0 / 18)$ \\
Jelšovce-Nitra & $0 \%$ & $(0 / 43)$
\end{tabular}

c) Sinusitis frontalis:

\begin{tabular}{lll} 
Jel.-Mad'arovce & $28,6 \%$ & $(2 / 7)$ \\
\hline Wandersleben & $8,3 \%$ & $(2 / 24)$ \\
İkiztepe & $6,3 \%$ & $(1 / 16)$ \\
Franzhausen-I & $0 \%$ & $(0 / 28)$
\end{tabular}

\section{e) Stomatitis:}

\begin{tabular}{lll} 
Wandersleben & $36,7 \%$ & $(18 / 49)$ \\
Jel.-Mad'arovce & $35,7 \%$ & $(10 / 28)$ \\
\hline Aiterhofen & $33,3 \%$ & $(2 / 6)$ \\
İkiztepe & $15,6 \%$ & $(10 / 64)$ \\
Franzhausen-I & $10,5 \%$ & $(6 / 57)$ \\
Hainburg & $0 \%$ & $(0 / 26)$
\end{tabular}

g) Hirndrucksymptomatik (MA + BZ):

\begin{tabular}{lll} 
Jel.-Mad'arovce & $34,8 \%$ & $(16 / 46)$ \\
\hline Hainburg & $17,1 \%$ & $(6 / 35)$ \\
Franzhausen-I & $10,1 \%$ & $(10 / 99)$ \\
Jel.-Aunjetitz & $9,7 \%$ & $(3 / 31)$ \\
İkiztepe & $5,1 \%$ & $(6 / 117)$ \\
Gemeinlebarn-F & $5 \%$ & $(1 / 20)$ \\
Pitten & $5 \%$ & $(1 / 20)$ \\
Jelšovce-Nitra & $2,3 \%$ & $(1 / 43)$ \\
Barbing & $0 \%$ & $(0 / 28)$ \\
Harting & $0 \%$ & $(0 / 22)$ \\
Boğazkale & $0 \%$ & $(0 / 64)$ \\
Pergamon & $0 \%$ & $(0 / 16)$
\end{tabular}

\section{b) Otitis media und}

\section{Pneumatisationshemmungen:}

\begin{tabular}{lll} 
Gemeinlebarn-F & $33,3 \%$ & $(9 / 27)$ \\
Hainburg & $28,9 \%$ & $(11 / 38)$ \\
Pitten & $26,3 \%$ & $(5 / 19)$ \\
Jel.-Mad'arovce & $23,1 \%$ & $(8 / 35)$ \\
\hline İkiztepe & $19,6 \%$ & $(10 / 51)$ \\
Jel.-Aunjetitz & $16 \%$ & $(2 / 24)$ \\
Franzhausen-I & $10,7 \%$ & $(5 / 56)$
\end{tabular}

\section{d) Otitis media:}

\begin{tabular}{llll} 
İkiztepe & & $16,3 \%$ & $(8 / 49)$ \\
Jel.-Mad'arovce & & $12,5 \%$ & $(3 / 24)$ \\
\hline Jel.-Aunjetitz & & $8 \%$ & $(2 / 24)$ \\
Jelšovce-Nitra & $0 \%$ & $(0 / 43)$
\end{tabular}

\section{f) Mastoiditis:}

\begin{tabular}{lll} 
İkiztepe & $21,7 \%$ & $(10 / 46)$ \\
Jel.-Aunjetitz & $12,5 \%$ & $(3 / 24)$ \\
Jelšovce-Nitra & $11,6 \%$ & $(5 / 43)$ \\
Jel.-Mad'arovce & $3 \%$ & $(1 / 33)$ \\
\hline
\end{tabular}

h) Hirndrucksymptomatik (nur BZ):

$\begin{array}{lll}\text { Jel.-Mad'arovce } & 34,8 \% & (16 / 46) \\ \text { Hainburg } & 17,1 \% & (6 / 35) \\ \text { Franzhausen-I } & 10,1 \% & (10 / 99) \\ \text { Jel.-Aunjetitz } & 9,7 \% & (3 / 31) \\ \text { İkiztepe } & 5,1 \% & (6 / 117) \\ \text { Gemeinlebarn-F } & 5 \% & (1 / 20) \\ \text { Pitten } & 5 \% & (1 / 20) \\ \text { Jelšovce-Nitra } & 2,3 \% & (1 / 43)\end{array}$


i) Meningeale Reizungen (MA + BZ):

\begin{tabular}{lll} 
Pergamon & $64,3 \%$ & $(9 / 14)$ \\
Barbing & $50 \%$ & $(11 / 22)$ \\
Harting & $45,5 \%$ & $(10 / 22)$ \\
Jel.-Mad'arovce & $26,7 \%$ & $(12 / 45)$ \\
\hline Boğazkale & $25 \%$ & $(16 / 64)$ \\
Franzhausen-I & $22,2 \%$ & $(22 / 99)$ \\
Jel.-Aunjetitz & $20,7 \%$ & $(6 / 29)$ \\
Hainburg & $17,1 \%$ & $(6 / 35)$ \\
Jelšovce-Nitra & $11,8 \%$ & $(5 / 43)$ \\
Gemeinlebarn -F & $10 \%$ & $(2 / 20)$ \\
Pitten & $10 \%$ & $(2 / 20)$ \\
İkiztepe & $9,4 \%$ & $(11 / 117)$
\end{tabular}

\section{k) Perisinusitis:}

\begin{tabular}{lll} 
Barbing & $27,3 \%$ & \\
Jel.-Mad'arovce & $26,2 \%$ & $(11 / 42)$ \\
\hline Jel.-Aunjetitz & $17,2 \%$ & $(5 / 31)$ \\
Harting & $13,6 \%$ & \\
Franzhausen-I & $9,8 \%$ & $(8 / 82)$ \\
Gemeinlebarn-F & $8,7 \%$ & $(2 / 23)$ \\
Jelšovce-Nitra & $7,0 \%$ & $(3 / 43)$ \\
Pitten & $5,3 \%$ & $(1 / 19)$ \\
İkiztepe & $2,8 \%$ & $(4 / 144)$ \\
Hainburg & $2,8 \%$ & $(1 / 36)$
\end{tabular}

(Jel.: Jelšovce, MA: Mittelalter, BZ: Bronzezeit)

\section{j) Meningeale Reizungen (nur BZ):}

Jel.-Mad'arovce $\quad 26,7 \% \quad(12 / 45)$

Franzhausen-I $\quad 22,2 \% \quad(22 / 99)$

Jel.-Aunjetitz $\quad 20,7 \% \quad(6 / 29)$

Hainburg $\quad 17,1 \% \quad(6 / 35)$

Jelšovce-Nitra $\quad 11,8 \% \quad(5 / 43)$

Gemeinlebarn-F $\quad 10 \% \quad(2 / 20)$

Pitten $\quad 10 \% \quad(2 / 20)$

İkiztepe $\quad 9,4 \% \quad(11 / 117)$ 
Abb. 25 Entzündliche Erkrankungen des Endocranialraums in den drei frühbronzezeitlichen Kinderpopulationen von Jelšovce (SCHULTZ et al. 1998)

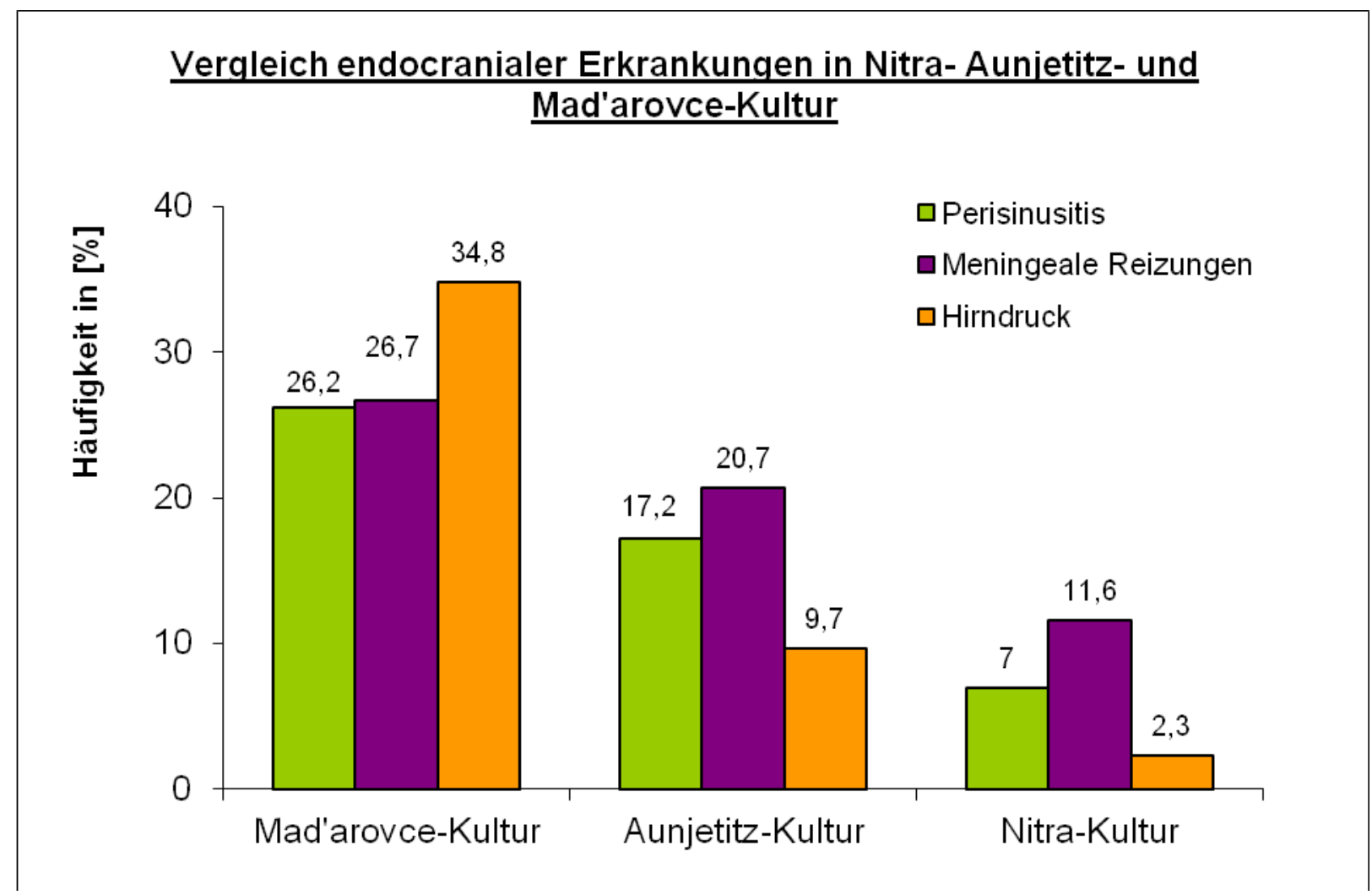

\begin{tabular}{|l|l|l|l|}
\hline & Mad'arocve-Kultur & Aunjetitz-Kultur & Nitra-Kultur \\
\hline Perisinusitis & $\mathrm{n}=11 / 42: 26,2 \%$ & $\mathrm{n}=5 / 31: 17,2 \%$ & $\mathrm{n}=3 / 43: 7 \%$ \\
\hline Meningeale Reizungen & $\mathrm{n}=12 / 45: 26,7 \%$ & $\mathrm{n}=6 / 29: 20,7 \%$ & $\mathrm{n}=5 / 43: 11,6 \%$ \\
\hline Hirndruck & $\mathrm{n}=16 / 46: 34,8 \%$ & $\mathrm{n}=3 / 31: 9,7 \%$ & $\mathrm{n}=1 / 43: 2,3 \%$ \\
\hline
\end{tabular}


Abb. 26 Vergleich endocranialer Erkrankungen verschiedener bronzezeitlicher und mittelalterlicher Kinderpopulationen (DETKEN 1989, POHL 1989, SCHULTZ 1987, 1993 und 2001 a, SCHULTZ \& SCHMIDT-SCHULTZ 1994, SCHULTZ et al. 1998)

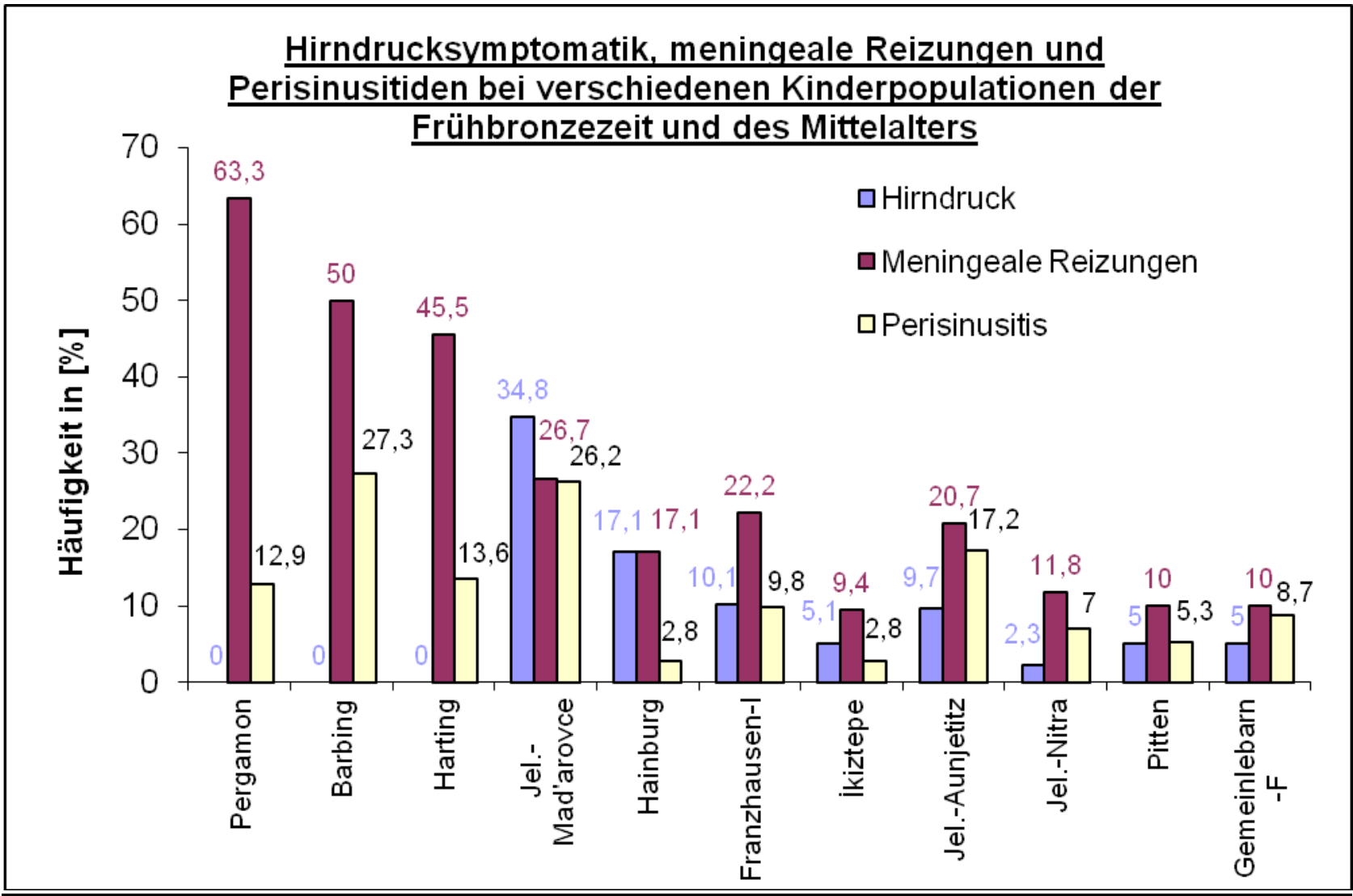

\begin{tabular}{|c|c|c|c|c|c|c|c|c|c|c|c|c|}
\hline & Per & Bar & Hart & MK & Hain & Fra-I & İkiztepe & $\mathrm{AK}$ & NK & Pit & Gm-F \\
\hline \multirow{2}{*}{ 旲 } & [\%] & 0 & 0 & 0 & 34,8 & 17,1 & 10,1 & 5,1 & 9,7 & 2,3 & 5 & 5 \\
\hline & abs. & $0 / 14$ & $0 / 22$ & $0 / 22$ & $16 / 46$ & $6 / 35$ & $10 / 99$ & $6 / 117$ & $3 / 31$ & $1 / 43$ & $1 / 20$ & $1 / 20$ \\
\hline \multirow{2}{*}{$\stackrel{q}{\Sigma}$} & [\%] & 64,3 & 50 & 45,5 & 26,7 & 17,1 & 22,2 & 9,4 & 20,7 & 11,8 & 10 & 10 \\
\hline & abs. & $9 / 14$ & $11 / 22$ & $10 / 22$ & $12 / 45$ & $6 / 35$ & $22 / 99$ & $11 / 117$ & $6 / 29$ & $5 / 43$ & $2 / 20$ & $2 / 20$ \\
\hline \multirow[b]{2}{*}{$\curvearrowleft$} & [\%] & 12,9 & 27,3 & 13,6 & 26,2 & 2,8 & 9,8 & 2,8 & 17,2 & 7,0 & 5,3 & 8,7 \\
\hline & abs. & $4 / 31$ & & & $11 / 42$ & $1 / 36$ & $8 / 82$ & $4 / 144$ & $5 / 31$ & $3 / 43$ & $1 / 19$ & $2 / 23$ \\
\hline
\end{tabular}

(Per: Pergamon, Bar: Barbing, Hart: Harting, MK: Mad'arovce-Kultur, Hain: Hainburg, Fra-I:

Franzhausen-I, AK: Aunjetitz-Kultur, NK: Nitra-Kultur, Pit: Pitten, Gm-F: Gemeinlebarn-F, HD: Hirndrucksymptomatik, MR: meningeale Reizungen, PS: Perisinusitis) 


\section{$\underline{\text { IV.5 Mangelerkrankungen }}$}

Ein Vergleich der Häufigkeiten der Mangelerkrankungen insgesamt bei den Populationen von Jelšovce zeigt das gleiche Bild wie bei den Entzündungserkrankungen (s. IV.4.). Die Kinder der Mad'arovce-Kultur sind von Mangelerkrankungen mit 66,1 \% (n = 39/ 59) insgesamt am häufigsten, die Kinder der Aunjetitz-Kultur mit 61,9 \% ( $n=26 / 42)$ etwas weniger und die Kinder der Nitra-Kultur mit 53,2 \% (n = 25/ 47) am wenigsten häufig betroffen (Tab. 25).

Bei den Kindern der Mad'arovce- und Aunjetitz-Kultur fallen im Vergleich zur Nitra-Kultur relativ viele Skorbutfälle auf. In Mad'arovce- und Aunjetitz-Kultur sind die Häufigkeiten mit $24,5 \%(n=12 / 49)$ und $23,3 \%(n=10 / 43)$ in etwa vergleichbar, in der Nitra-Kultur sind es nur 12,8 \% ( $n=6 / 47)$. Bei den übrigen Mangelerkrankungen zeigt sich eine andere Verteilung. Anämie und Rachitis treten bei den Kindern der Aunjetitz-Kultur mit 38,7 \% (n = 12/ 31) bzw. 7,1 \% ( $=3 / 42)$ am häufigsten auf, gefolgt von den Kindern der Nitra-Kultur mit 17,0 \% ( $\mathrm{n}=8 / 47)$ bzw. 2,1 \% $(\mathrm{n}=1 / 48)$. Hier sind die Mad'arovce-Kinder mit 10,6 \% $(\mathrm{n}=$ 5/ 47) bzw. $0 \%(n=0 / 58)$ am seltensten betroffen. Nur bei einem Kind der Mad'arovceKultur besteht der Verdacht auf eine ausgeheilte Rachitis ( $n=1 / 58: 1,7 \%)$. In allen drei Kulturen treten bei einem bis drei Kindern sowohl Anämie als auch Skorbut bei demselben Individuum auf.

Wie Abbildung 28 zeigt, weisen Aunjetitz- und Nitra-Kultur für Mangelerkrankungen ebenso wie für die Entzündungserkrankungen ein entsprechendes Krankheitsprofil auf (vgl. IV.4.). In beiden Populationen ist die Anämie am häufigsten, gefolgt von Skorbut und Rachitis (Abb. 27 und 28). In der Aunjetitz-Kultur liegt die Verteilung auf einem etwas höheren Niveau als in der Nitra-Kultur. In der Mad'arovce-Kultur sieht dieses Profil anders aus. Auch hier tritt die Rachitis am seltensten auf; Skorbutfälle jedoch häufiger als Fälle von Anämien (Abb. 27 und 28). Nach dem in Aunjetitz-Kultur und Nitra-Kultur erkennbaren Krankheitsprofil würde man 
in der Mad'arovce-Kultur bei gleichem Profil eine deutlich niedrigere Rate an Skorbuterkrankungen erwarten (etwa 6 - $7 \%$ ).

Die Rachitis scheint in der Bronzezeit in Mitteleuropa eine seltene Erkrankung gewesen zu sein. Ebenso wie in Jelšovce-Mad'arovce treten in Franzhausen-I und Pitten sowie den neolithischen Populationen von Wandersleben und Aiterhofen keine Fälle auf. Auch in den anderen bronzezeitlichen Populationen handelt es sich nur um Einzelfälle. In Jelšovce-Nitra und Hainburg ist jeweils nur ein Kind betroffen, in İkiztepe und Jelšovce-Aunjetitz liegt die Häufigkeit bei 3,9 \% ( $\mathrm{n}=$ 5/ 129: 3,9 \%) bzw. 7,1 \% ( $\mathrm{n}=3 /$ 42: 7,1 \%) (Abb. 29, Tab. 27). Der Skorbut hingegen war weiter verbreitet. Vergleichbar hoch wie in Jelšovce-Mad'arovce und -Aunjetitz, wo jeweils etwa ein Viertel der Kinder betroffen ist, sind die Zahlen bei anderen bronzezeitlichen Populationen allerdings nicht. In İkiztepe sind 13,8 \% ( $\mathrm{n}=17 / 123)$, in Pitten 8,7 \% und in Franzhausen-I 6,4 \% $(\mathrm{n}=7 / 110)$ der Kinder betroffen. Aus Hainburg ist kein einziger Fall bekannt $(n=0 / 32)$. Anders sieht es für mittelalterliche Populationen aus; in Straubing litten fast $36 \%(n=57 / 159:$ 35,8 \%) der Kinder an Skorbut (Abb. 30, Tab. 26) (s. IV.7).

Ein anderes Verteilungsmuster zeigt die Anämie. Hier sind die Kinder aus Jelšovce-Aunjetitz mit fast $39 \%(n=12 / 31)$ verglichen mit allen anderen bronzezeitliche Populationen mit Abstand am häufigsten betroffen, gefolgt von den Kindern aus Jelšovce-Nitra mit 17 \% ( $n=8$ / 47). In Franzhausen-I, Hainburg und Gemeinlebarn-F treten überhaupt keine Fälle von Anämien auf. In İkiztepe sind nur 4,7 \% $(n=6 / 129)$ der Kinder betroffen, in Wandersleben sind es 12,9 \% ( $n=11 / 85)$, in Jelšovce-Mad'arovce 10,6 \% $(n=5 / 47)$ (Abb. 31, Tab. 28).

Stressmarker, die unter anderem mit Mangelerkrankungen in Verbindung gebracht werden, sind Harris-Linien, transversale Schmelzhypoplasien, Cribra orbitalia und poröse Oberflächen an Langknochen. Sie gelten als unspezifische Zeichen einer Wachstumsstörung des Kindes, sofern Orbitae und Langknochen nicht mikroskopisch untersucht werden konnten, und können auch im Rahmen von entzündlichen Erkrankungen auftreten. 
Die Ursachen der Wachstumsstörung sind aus den genannten Veränderungen nicht zu erkennen. Innerhalb der Kinderpopulationen aus Jelšovce stehen lediglich Vergleichsdaten für die transversalen Schmelzhypoplasien zur Verfügung. Die Häufigkeiten in Aunjetitz- und Mad'arovce-Kultur entsprechen sich mit 60,9 \% $(n=25 / 41)$ bzw. 60,8 \% $(n=31 / 51)$. Die Belastung in der Nitra-Kultur ist mit 38,3 \% $(n=18 / 47)$ deutlich geringer (Tab. 30). Es zeigt sich die gleiche Verteilung wie für Skorbut (s.o.) (Tab. 25 und 26). In Wandersleben und Franzhausen-I treten transversale Schmelzhypoplasien ähnlich häufig auf wie in JelšovceMad'arovce und -Aunjetitz ( $n=49 /$ 87: 56,3 \% bzw. $n=43 / 68: 63,2 \%$ ). In Hainburg, Gemeinlebarn-F und İkiztepe sind vergleichbar mit Jelšovce-Nitra zwischen 30 und 40 \% der Kinder betroffen (Tab. 29). Niedrig ist der Befall in Pitten mit nur 18,2 \% $(n=4 / 22)$ (Tab. 29). Insgesamt sind transversale Schmelzhypoplasien im Neolithikum häufiger als in der Frühbronzezeit. Gegen Ende der Frühbronzezeit sind sie wiederum häufiger als zu ihrem Beginn.

Für Harris-Linien liegen lediglich Daten für Jelšovce-Mad'arovce und Franzhausen-I vor. Für Franzhausen-I wird eine Häufigkeit von etwa 40 \% angegeben. In Jelšovce-Mad'arovce sind mit 89,5 \% ( $\mathrm{n}=17 / 19)$ deutlich mehr Kinder betroffen.

Poröse Orbitadächer (Cribra orbitalia) sind in Jelšovce-Mad'arovce mit 62,5 \% (n = 20/ 32) sehr häufig. In allen anderen verglichenen Populationen ist das Auftreten seltener, obwohl auch in Wandersleben mit 53,7 \% $(n=29 / 54)$, in İkiztepe mit 52,4 \% $(n=43 / 82)$ und Franzhausen-I mit 47,4 \% ( $\mathrm{n}=27 / 57)$ viele Kinder betroffen sind (Tab. 31). Etwas weniger häufig treten Cribra orbitalia in Boğazkale auf $(n=7 / 19: 36,8 \%)$. Selten sind sie in Hainburg mit nur 6,3 \% $(n=2 / 32)$. In allen Populationen überwiegt der Grad I, nur in Jelšovce-Mad'arovce und in İkiztepe treten relativ häufig auch Grad II und III auf $(n=9 / 32: 28,1 \%$ bzw. $n=14$ / 82: 17,1 \%; Tab. 31). In Gemeinlebarn-F treten bei 12,5 \% $(n=2 / 16)$, in Franzhausen-I bei $8,8 \%(\mathrm{n}=5 / 57)$ und in Boğazkale bei 5,3 \% $(\mathrm{n}=1 / 19)$ der Kinder Cribra orbitalia vom Grad II auf (Tab. 31). 
Zu porösen Oberflächen an Langknochen liegen keine Vergleichsdaten vor.

Insgesamt zeigt sich für die Mangelerkrankungen ein ähnliches Bild wie für die Entzündungserkrankungen (s. IV.4). Die bronzezeitlichen Populationen aus dem Nitratal sind stärker von Mangelerkrankungen betroffen als die etwa zeitgleich lebenden Populationen in Niederösterreich. Besonders für Anämie und Skorbut wird dieser Unterschied deutlich. Nur die mittelalterlichen Populationen sind noch stärker betroffen. Bei der Rachitis handelt es sich überwiegend um Einzelfälle. Unspezifische Stressmarker treten in Jelšovce-Mad'arovce häufiger auf als in den übrigen bronzezeitlichen Populationen.

Tab. 25 Die Häufigkeiten von verschiedenen Mangelerkrankungen in Nitra-, Aunjetitz- und Mad'arovce-Kultur von Jelšovce (in Prozent). (ScHULTZ et al. 1998)

\begin{tabular}{|c|c|c|c|}
\hline & \multicolumn{3}{|c|}{ Häufigkeit in Prozent [\%] } \\
\hline & $\begin{array}{l}\text { Mad'arovce- } \\
\text { Kultur }\end{array}$ & $\begin{array}{l}\text { Aunjetitz- } \\
\text { Kultur }\end{array}$ & $\begin{array}{l}\text { Nitra- } \\
\text { Kultur }\end{array}$ \\
\hline Mangelerkr. ges. & $66,1 \quad(39 / 59)$ & $61,9 \quad(26 / 42)$ & $53,2(25 / 47)$ \\
\hline Skorbut & $24,5 \quad(12 / 49)$ & $23,3 \quad(10 / 43)$ & $12,8(6 / 47)$ \\
\hline Anämie & $10,6 \quad(5 / 47)$ & $38,7 \quad(12 / 31)$ & $17 \quad(8 / 47)$ \\
\hline Rachitis & $(0 / 58)$ & $7,1 \quad(3 / 42)$ & $2,1 \quad(1 / 48)$ \\
\hline
\end{tabular}

(Mangelerkr.: Mangelerkrankungen; ges.: gesamt) 
Abb. 27 Das Auftreten von Anämie, Rachitis und Skorbut in den frühbronzezeitlichen Kinderpopulationen von Jelšovce (Häufigkeit in Prozent). (SCHULTZ et al. 1998)

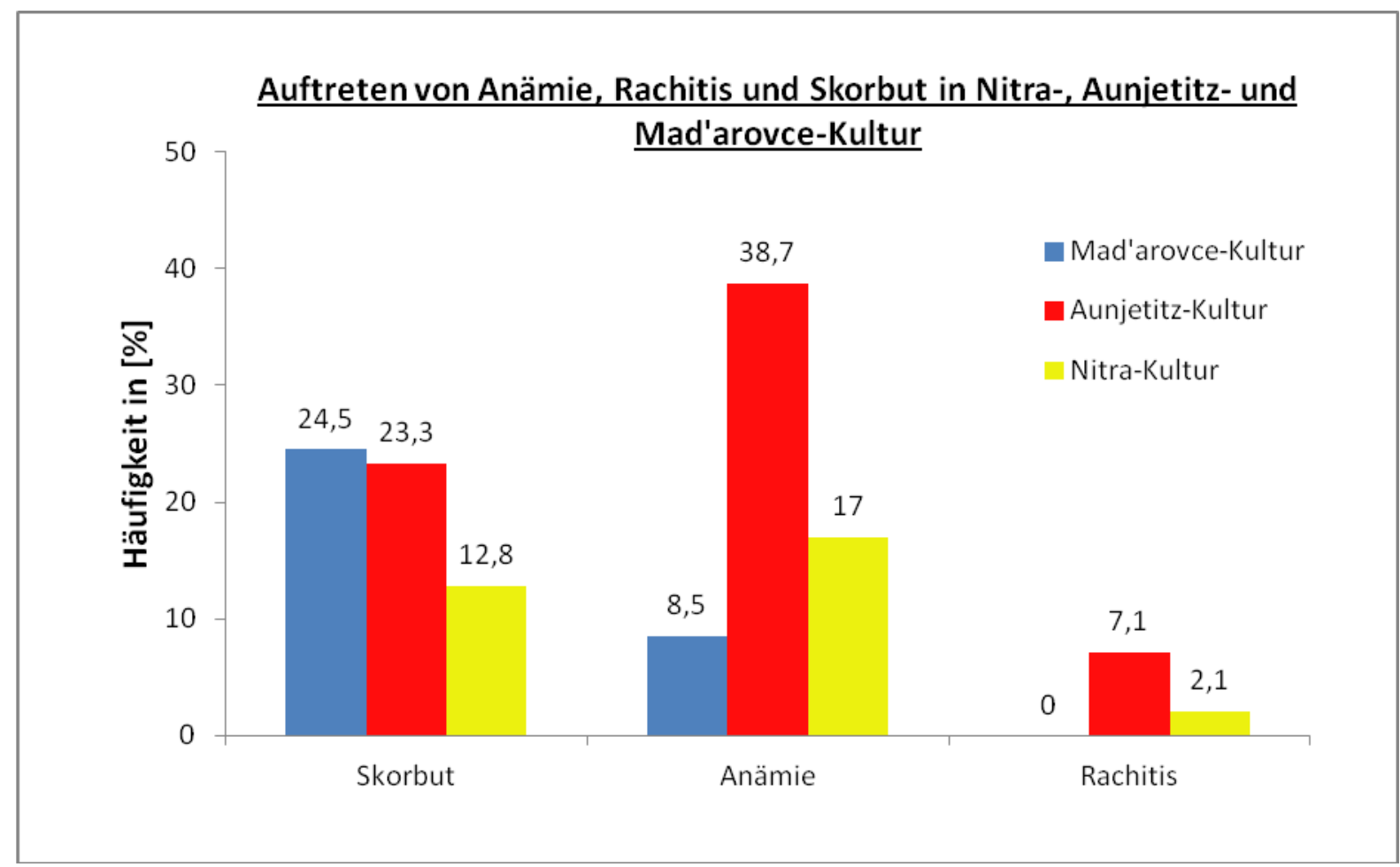

\begin{tabular}{|l|l|l|l|}
\hline & Skorbut & Anämie & Rachitis \\
\hline Mad'arovce-Kultur & $\mathrm{n}=12 / 49: 24,5 \%$ & $\mathrm{n}=5 / 47: 8,5 \%$ & $\mathrm{n}=0 / 58: 0 \%$ \\
\hline Aunjetitz-Kultur & $\mathrm{n}=10 / 43: 23,3 \%$ & $\mathrm{n}=12 / 31: 38,7 \%$ & $\mathrm{n}=3 / 42: 7,1 \%$ \\
\hline Nitra-Kultur & $\mathrm{n}=6 / 47: 12,8 \%$ & $\mathrm{n}=8 / 47: 17,0 \% \%$ & $\mathrm{n}=1 / 47: 2,1 \%$ \\
\hline
\end{tabular}




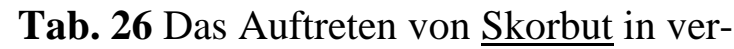
schiedenen bronzezeitlichen, neolithischen und mittelalterlichen Kinderpopulationen.

\begin{tabular}{|l|lc|}
\hline Kultur & $\begin{array}{l}\text { Häufigkeit in } \\
\text { Prozent [\%] }\end{array}$ \\
\hline Straubing & $35,6 \quad(57 / 159)$ \\
\hline Wandersleben & $34,9 \quad(22 / 68)$ \\
\hline Jel.-Mad'arovce & $24,5 \quad(12 / 49)$ \\
\hline Jel.-Aunjetitz & $23,3 \quad(10 / 43)$ \\
\hline İkiztepe & $13,8 \quad(17 / 123)$ \\
\hline Jelšovce-Nitra & $12,8 \quad(6 / 47)$ \\
\hline Pitten & 8,7 \\
\hline Franzhausen-I & $6,4 \quad(7 / 110)$ \\
\hline Hainburg & 0 & $(0 / 32)$ \\
\hline
\end{tabular}

Tab. 28 Das Auftreten von Anämie in verschiedenen bronzezeitlichen und neolithischen Populationen.

\begin{tabular}{|l|ll|}
\hline Kultur & \multicolumn{2}{|l|}{$\begin{array}{l}\text { Häufigkeit in } \\
\text { Prozent [\%] }\end{array}$} \\
\hline Jel.-Aunjetitz & 38,7 & $(12 / 31)$ \\
\hline Jelšovce-Nitra & 17 & $(8 / 47)$ \\
\hline Wandersleben & 12,9 & $(11 / 85)$ \\
\hline Jel.-Mad'arovce & 8,5 & $(4 / 47)$ \\
\hline İkiztepe & 4,7 & $(6 / 129)$ \\
\hline Franzhausen-I & 0 & $(0 / 52)$ \\
\hline Hainburg & 0 & $(0 / 30)$ \\
\hline Gemeinlebarn-F & 0 & $(0 / 18)$ \\
\hline
\end{tabular}

Tab. 27 Das Auftreten von Rachitis in verschiedenen bronzezeitlichen und neolithischen Kinderpopulationen.

\begin{tabular}{|l|lc|}
\hline Kultur & \multicolumn{2}{|l|}{$\begin{array}{l}\text { Häufigkeit in } \\
\text { Prozent [\%] }\end{array}$} \\
\hline Jel.-Aunjetitz & 7,1 & $(3 / 42)$ \\
\hline İkiztepe & 3,9 & $(5 / 129)$ \\
\hline Hainburg & 2,6 & $(1 / 38)$ \\
\hline Jelšovce-Nitra & 2,1 & $(1 / 47)$ \\
\hline Jel.-Mad'arovce & 0 & $(0 / 58)$ \\
\hline Franzhausen-I & 0 & $(0 / 110)$ \\
\hline Pitten & 0 & $(0 / 32)$ \\
\hline Wandersleben & 0 & $(0 / 98)$ \\
\hline Aiterhofen & 0 & $(0 / 27)$ \\
\hline
\end{tabular}

Tab. 29 Das Auftreten von transversalen Schmelzhypoplasien in verschiedenen bronzezeitlichen und neolithischen Populationen.

\begin{tabular}{|l|ll|}
\hline Kultur & \multicolumn{2}{|l|}{ Häufigkeit in } \\
& Prozent [\%] \\
\hline Wandersleben & $63,2 \quad(43 / 68)$ \\
\hline Jel.-Aunjetitz & $60,9 \quad(25 / 41)$ \\
\hline Jel.-Mad'arovce & $60,8 \quad(31 / 51)$ \\
\hline Franzhausen-I & $56,3 \quad(49 / 87)$ \\
\hline Jelšovce-Nitra & $38,3 \quad(18 / 47)$ \\
\hline Hainburg & 36,1 & $(13 / 36)$ \\
\hline Gemeinlebarn-F & $34,9 \quad(15 / 43)$ \\
\hline İkiztepe & $28,7 \quad(16 / 88)$ \\
\hline Pitten & $18,2 \quad(4 / 22)$ \\
\hline
\end{tabular}

(Tab. 26-29: CARLi-THIELE 1996, CARLI-THIELE \& SCHUlTZ 2001, KREUTZ 1997, SCHULTZ 2001 a, SCHULTZ et al. 1998) 
Tab. 30 Das Auftreten von transversale Schmelzhypoplasien bei den Kinderpopulationen von Nitra-, Aunjetitz- und Mad'arovce-Kultur in Jelšovce. (SCHULTZ et al. 1998)

\begin{tabular}{|l|lc|}
\hline Kultur & $\begin{array}{l}\text { Häufigkeit für transversale } \\
\text { Schmelzhypoplasien in } \\
\text { Prozent [\%] }\end{array}$ \\
\hline Aunjetitz & 60,9 & $(25 / 41)$ \\
\hline Mad'arovce & 60,8 & $(31 / 51)$ \\
\hline Nitra & 38,3 & $(18 / 47)$ \\
\hline
\end{tabular}

Abb. 28 Häufigkeitsverteilung von Anämie, Rachitis und Skorbut in den drei frühbronzezeitlichen Kinderpopulationen von Jelšovce (Häufigkeit in Prozent). (SCHULTZ et al. 1998)

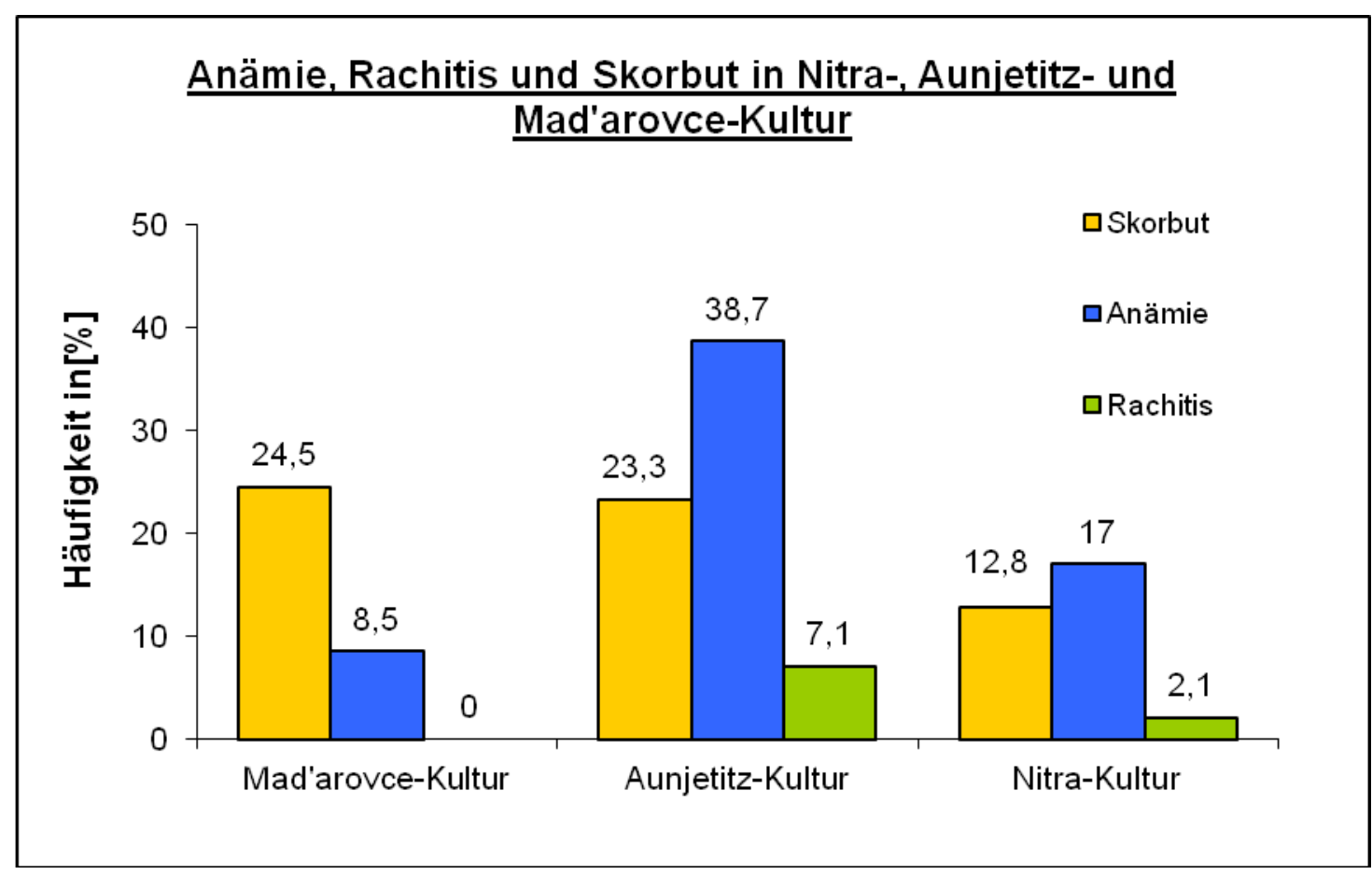

\begin{tabular}{|l|l|l|l|}
\hline & Mad'arovce-Kultur & Aunjetitz-Kultur & Nitra-Kultur \\
\hline Skorbut & $\mathrm{n}=12 / 49: 24,5 \%$ & $\mathrm{n}=10 / 43: 23,3 \%$ & $\mathrm{n}=6 / 47: 12,8 \%$ \\
\hline Anämie & $\mathrm{n}=5 / 47: 8,5 \%$ & $\mathrm{n}=12 / 31: 38,7 \%$ & $\mathrm{n}=8 / 47: 17,0 \% \%$ \\
\hline Rachitis & $\mathrm{n}=0 / 58: 0 \%$ & $\mathrm{n}=3 / 42: 7,1 \%$ & $\mathrm{n}=1 / 47: 2,1 \%$ \\
\hline
\end{tabular}


Tab. 31 Das Auftreten von Cribra orbitalia und transversalen Schmelzhypoplasien in verschiedenen bronzezeitlichen und neolithischen Populationen.

(CARli-Thiele \& Schultz 2001, Schultz 1989 b, Schultz 1988/89, SCHUltz 1990, SCHULTZ 2001 a)

\begin{tabular}{|c|c|c|c|c|c|c|c|}
\hline \multirow{3}{*}{ Kultur } & \multicolumn{6}{|c|}{ Cribra orbitalia } & \multirow{3}{*}{$\begin{array}{l}\text { transversale } \\
\text { Schmelzhypo- } \\
\text { plasien, } \\
\text { Häufigkeit in [\%] }\end{array}$} \\
\hline & \multicolumn{3}{|c|}{$\begin{array}{l}\text { Anzahl der erkrankten } \\
\text { Individuen (absolut) }\end{array}$} & \multicolumn{2}{|c|}{$\begin{array}{l}\text { Anzahl der } \\
\text { Individuen } \\
\text { (absolut) }\end{array}$} & \multirow[t]{2}{*}{$\begin{array}{l}\text { Anzahl der } \\
\text { erkrankten } \\
\text { Individuen } \\
\text { gesamt in [\%] }\end{array}$} & \\
\hline & Grad I & Grad II & Grad III & krank & gesund & & \\
\hline Jel.-Mad'arovce & 11 & 7 & 2 & 20 & 12 & 62,5 & $60,8 \quad(31 / 51)$ \\
\hline İkiztepe & 29 & 10 & 4 & 43 & 39 & 52,4 & $28,7 \quad(25 / 87)$ \\
\hline Franzhausen-I & 22 & 5 & 0 & 27 & 30 & 47,4 & $56,3 \quad(49 / 87)$ \\
\hline Wandersleben & & & & 29 & 25 & 53,7 & $63,2 \quad(43 / 68)$ \\
\hline Boğazkale & 6 & 1 & 0 & 7 & 12 & 36,8 & $50 \quad(17 / 34)$ \\
\hline Gemeinlebarn-F & 3 & 2 & 0 & 5 & 11 & 31,3 & $34,9 \quad(15 / 43)$ \\
\hline Pitten & 3 & 0 & 0 & 3 & 9 & 25 & $18,2 \quad(4 / 22)$ \\
\hline Hainburg & & & & 2 & 30 & 6,3 & $36,1 \quad(13 / 36)$ \\
\hline
\end{tabular}

Abb. 29 Das Auftreten von Rachitis in verschiedenen bronzezeitlichen und neolithischen Kinderpopulationen (Häufigkeit in Prozent).

(CARLI-THIELE \& SCHULTZ 2001, SCHULTZ 2001 a, SCHULTZ et al. 1998)

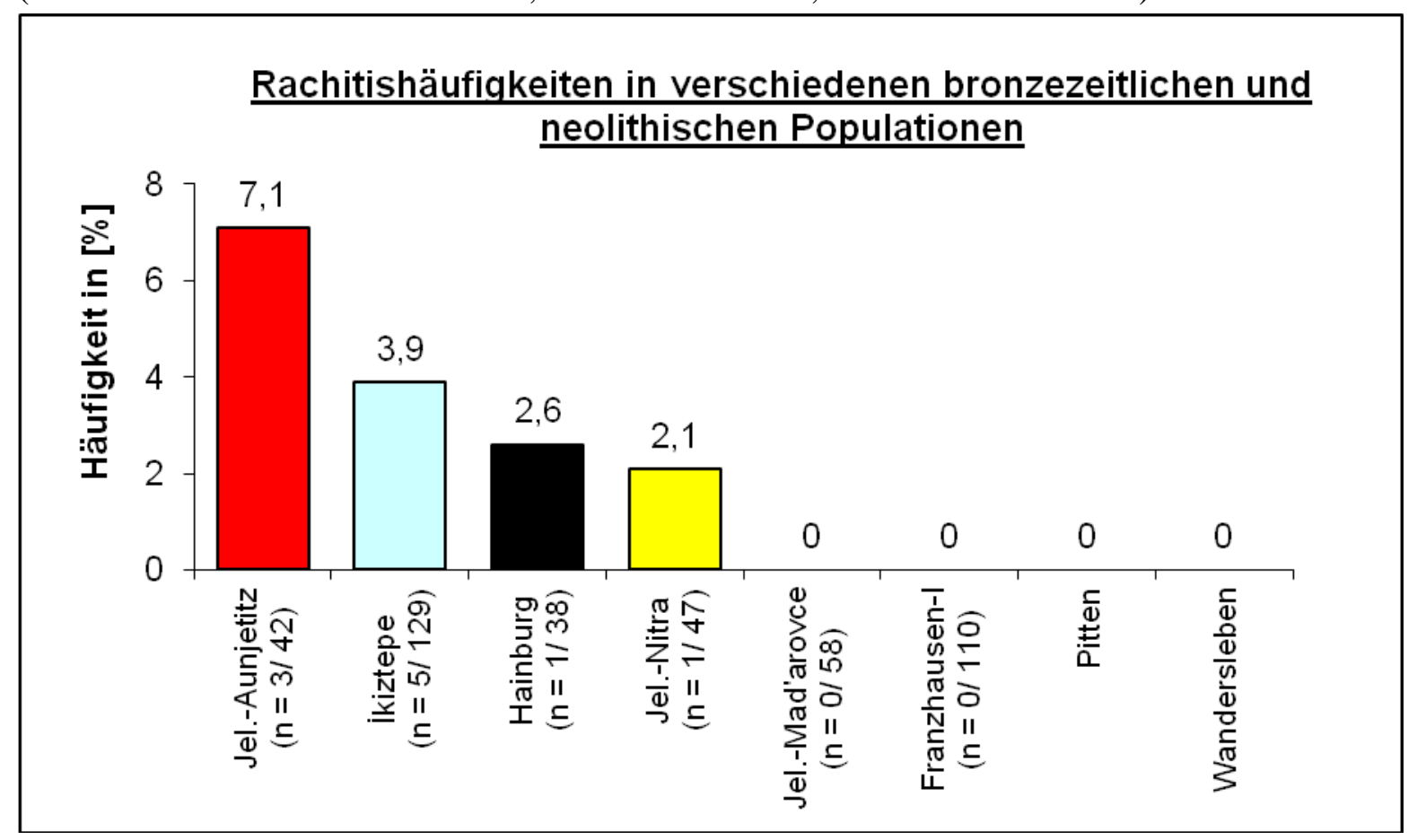

(Jel.: Jelšovce) 
Abb. 30 Das Auftreten von Skorbut in verschiedenen bronzezeitlichen und neolithischen

Kinderpopulationen (Häufigkeit in Prozent).

(CARLI-ThiElE \& SChUltz 2001, KREUTZ 1997, SChUltZ 2001 a, SCHUltz et al. 1998)

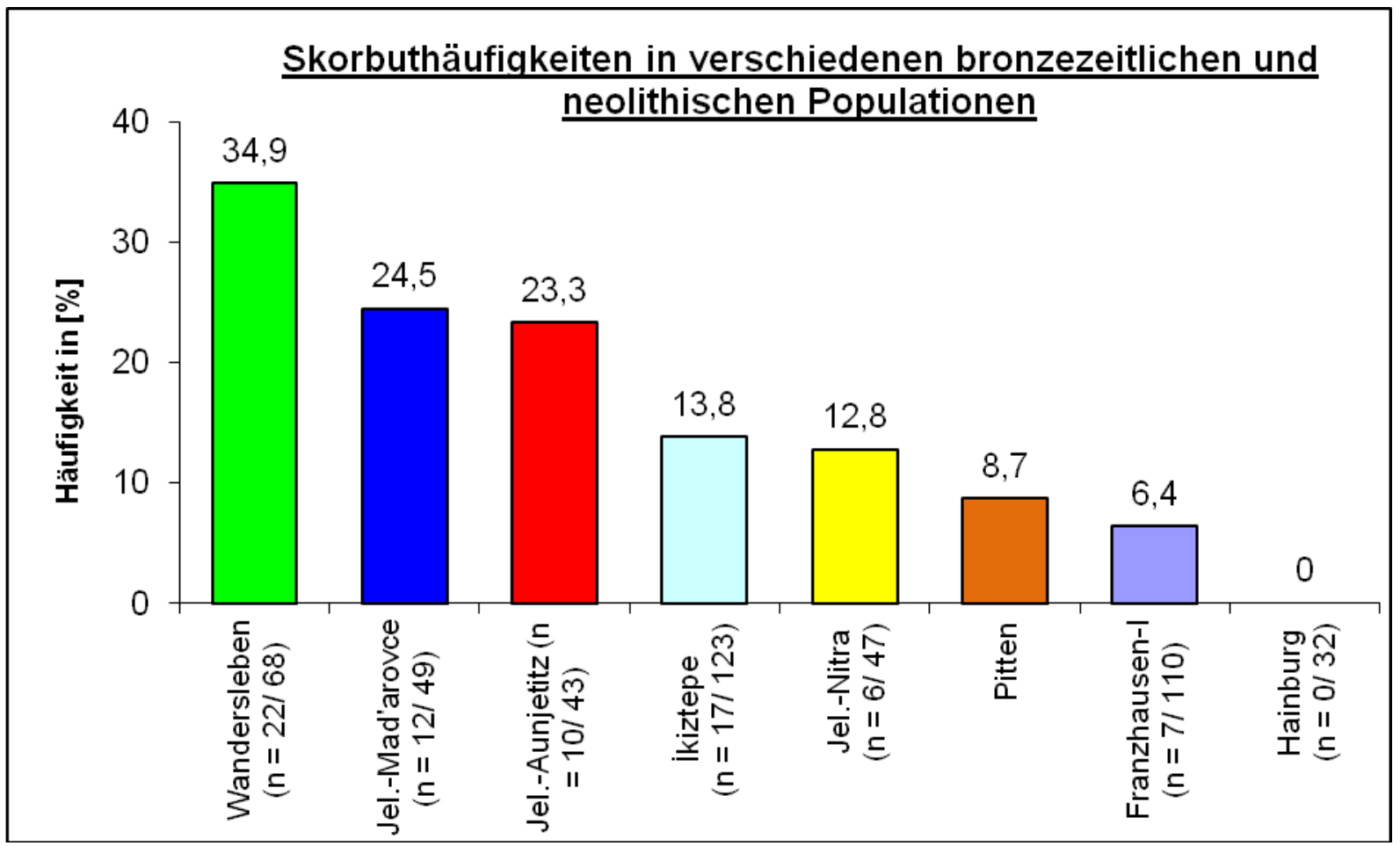

(Jel.: Jelšovce)

Abb. 31 Das Auftreten von Anämien in verschiedenen bronzezeitlichen und neolithischen

Kinderpopulationen (Häufigkeit in Prozent).

(CARLI-THIELE \& SCHUltz 2001, KREUTZ 1997, SCHUlTZ 2001 a, SCHUlTZ et al. 1998)

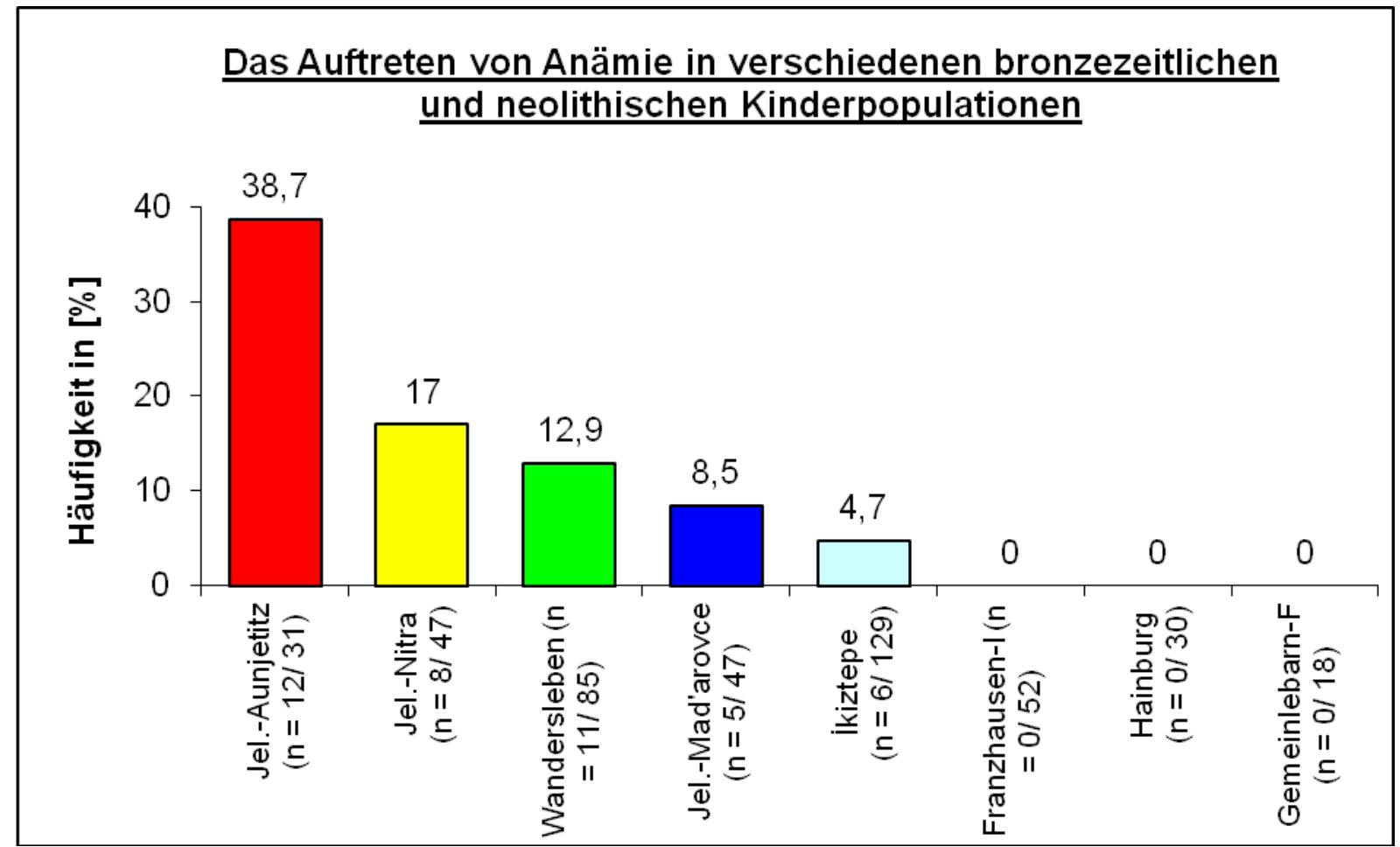

(Jel.: Jelšovce) 


\section{IV.6 Zusammenfassende Darstellung der Vergleichsergebnisse}

Die Altersverteilung in Jelšovce zeigt ein relatives Kleinkinderdefizit. In allen drei Populationen lassen sich mehr Kinder der Gruppe Infans II als Infans I zuordnen. Kinder der Gruppe Infans Ia sind besonders in Nitra- und Aunjetitz-Kultur kaum vertreten (5 - 8 \%). Es zeigt sich ein Rückgang der Kindersterblichkeit im Verlauf der Frühbronzezeit, der mit einer Verschiebung der Sterblichkeit in jüngere Altersklassen einher geht. Alle drei Populationen aus Jelšovce hatten verglichen mit mittelalterlichen Kinderpopulationen bessere Überlebenschancen. Besonders in den ersten vier Lebensjahren verstarben in der Bronzezeit anteilig weniger Kinder als im Mittelalter.

Sowohl in bezug auf entzündliche Erkrankungen des Schädels als auch auf Mangel- und Zahnerkrankungen insgesamt sind die Kinder der Mad'arovce-Kultur im Vergleich zu den anderen frühbronzezeitlichen Populationen von Jelšovce am häufigsten betroffen, gefolgt von den Kindern der Aunjetitz-Kultur. Die Kinder der Nitra-Kultur weisen die niedrigsten Erkrankungsraten auf.

Ausnahmen bilden Anämie, Rachitis, Karies und Mastoiditis. Diese treten bei den Kindern der Aunjetitz-Kultur am häufigsten, bei denen der Mad'arovce-Kultur am seltensten auf. Von einem Skorbut ebenso wie von transversalen Schmelzhypoplasien sind Aunjetitz- und Mad'arovce-Kultur etwa gleich häufig betroffen, die Kinder der Nitra-Kultur deutlich weniger.

Im Vergleich mit anderen bronzezeitlichen Populationen zeigt sich, dass fast alle Entzündungserkrankungen bei den Kindern der Mad'arovce-Kultur am häufigsten auftreten. Eine Ausnahme bildet Otitis media mit Pneumatisationshemmungen (und Mastoiditis). Von Mittelohrerkrankungen sind Gemeinlebarn-F, Hainburg und Pitten (26 - 33 \%) häufiger als Jelšovce-Mad'arovce betroffen ( $\mathrm{n}=$ 9/ 39: 23,1 \%). Verglichen mit mittelalterlichen Populationen treten meningeale Erkrankungen bei allen frühbronzezeitlichen Populationen deutlich seltener auf (45 - 60 \% versus 10 - 27 \%). In Jelšovce-Mad'arovce sind sie häufiger als in den 
übrigen bronzezeitlichen Populationen. Außerdem zeigt sich, dass diese bei den gegen Ende der Frühbronzezeit lebenden Populationen häufiger auftreten als bei denjenigen, die zu deren Beginn lebten. Gleiches gilt auch für Zahnerkrankungen. Einerseits sind die mittelalterlichen häufiger als die bronzezeitlichen Populationen betroffen, andererseits kam es zu einer Zunahme der Erkrankungshäufigkeiten im Verlauf der Frühbronzezeit.

In bezug auf die Mangelerkrankungen muss stärker differenziert werden. Anämie und Rachitis treten in Jelšovce-Aunjetitz häufiger als in allen anderen Populationen auf. In allen drei Populationen von Jelšovce sind Anämien häufiger als in den anderen frühbronzezeitlichen Populationen. Rachitis tritt nur in Einzelfällen auf. Lediglich in Jelšovce-Aunjetitz und İkiztepe ist mehr als ein Kind betroffen $(n=3 / 42: 7,1 \%$ bzw. $n=5 / 129: 3,9 \%)$. Skorbut tritt im Mittelalter (z.B. in Straubing) häufiger auf als in der Bronzezeit. In Jelšovce-Aunjetitz und -Mad'arovce ist der Skorbut ebenso wie transversale Schmelzhypoplasien häufiger als in den übrigen bronzezeitlichen Populationen. Für transversale Schmelzhypoplasien lässt sich eine Zunahme der Erkrankungshäufigkeiten im Verlauf der Frühbronzezeit feststellen. Im Neolithikum traten sie vergleichbar häufig auf wie gegen Ende der Frühbronzezeit. Für Cribra orbitalia zeigt sich, dass die Kinder aus Jelšovce-Mad'arovce ebenfalls häufiger als die Kinder der übrigen Populationen betroffen sind.

\section{IV.7 Ursachendiskussion der beobachteten Ergebnisse}

Im Verlauf der Frühbronzezeit ist es zu einer Verschlechterung der Lebensbedingungen in der heutigen Slowakei und in Niederösterreich gekommen. Ein Grund dafür ist offenbar in dem sich wandelnden Klima in der Frühbronzezeit zu sehen (vgl. IV.1.1). Die kühler und trockener werdende Klimalage führte zu einem Rückgang der landwirtschaftlichen Erträge. Besonders durch die starken Klimaschwankungen stieg das Risiko für Missernten an. Die daraus resultierende schlechtere Ernährungslage führte zu vermehrtem Auftreten von Mangelernäh- 
rung und Mangelerkrankungen. Eine Mangelernährung, z.B. der Mangel an Proteinen und Vitaminen, führt zu einer Schwächung des Immunsystems, besonders bei Kindern, und damit zu einer Zunahme der Infektanfälligkeit. Das kühlere Klima an sich begünstigte ebenfalls die Ausbreitung von Entzündungserkrankungen, besonders in den pneumatischen Räumen des Schädels.

Als Folge der oben genannten Klimaveränderungen ist eine Zunahme der Erkrankungshäufigkeiten von Entzündungs-, Mangel- und Zahnerkrankungen gegen Ende der Frühbronzezeit zu beobachten. Jelšovce-Mad'arovce ist als Vertreter der späten Frühbronzezeit sowohl von fast allen Entzündungs-, als auch Zahnerkrankungen am häufigsten betroffen (Tab. 18 und 24). Die ebenfalls in die späte Frühbronzezeit datierte Population von Hainburg weist für Parodontopathien und endocraniale Erkrankungen ebenfalls vergleichsweise hohe Werte auf (Tab. 20 und 24 g - j). Im Gegensatz dazu traten bei den absolut früher datierten Populationen aus Franzhausen-I, Gemeinlebarn-F, Jelšovce-Nitra und -Aunjetitz bei noch besseren klimatischen Bedingungen weniger dieser Erkrankungen auf.

Als Ursachen für die hohe Erkrankungsbelastung der Kinder der Mad'arovce-Kultur müssen aber auch weitere Faktoren diskutiert werden. Die Zusammenhänge zwischen Wohnverhältnissen für das Auftreten von Nasennebenhöhlen- und Ohrenerkrankungen sind von Schultz (1982) ausführlich diskutiert worden. Zugige, kalte, feuchte Behausungen führten zu ständiger Reizung der Schleimhäute und daraus resultierend zu einer chronischen Entzündung (SCHUltz 1982 und 1989 c). Wie die Behausungen der Menschen der Mad'arovce-Kultur genau aussahen, ist noch Gegenstand archäologischer Forschungen. Es ist aber davon auszugehen, dass diese vor allem im Winter nicht immer warm und trocken waren. Diesbezüglich bestanden keine wesentlichen Unterschiede zwischen den verschiedenen slowakischen und niederösterreichischen Populationen. Im deutlich südlicher gelegenen İkiztepe sind Entzündungserkrankungen mit Ausnahme von Mittelohrentzündungen vergleichsweise selten. Erklärung hierfür sind wahrscheinlich die unterschiedlichen klimatischen Bedingungen. Erstens 
liegt İkiztepe in der Zone des mediterranen, maritimen und damit milderen Klimas. Zweitens wird die Siedlung absolut früher datiert. Es herrschte noch ein insgesamt wärmeres und stabileres Klima vor. In kalten, feuchten Klimazonen sind Erkältungserkrankungen bekanntlich weiter verbreitet als in warmen, trockenen Zonen.

Auch die Größe einer Population und das Ausmaß von Kontakten zu benachbarten Gruppen spielt eine wichtige Rolle bei der Übertragung und Verbreitung von Infektionserkrankungen. Kontakte zu benachbarten Kulturen sind für alle Populationen nachweisbar. Mögliche Ursachen des Anstiegs von Erkrankungen in Jelšovce-Mad'arovce sind daher in der Zunahme der Populationsgröße verglichen mit Jelšovce-Nitra und -Aunjetitz zu suchen. Je mehr Menschen an einem Ort zusammen leben, desto mehr Ansteckungsquellen für entzündliche Erkrankungen gibt es auch. Die Tatsache, dass in den ebenfalls relativ großen Populationen von İkiztepe und Franzhausen relativ gesehen weniger Infektionserkrankungen auftreten, ist vermutlich auf die oben genannten klimatischen Bedingungen zurückzuführen.

Das Zusammenleben mit Tieren birgt ebenfalls Infektionsquellen. Die Übertragung von Krankheiten von Tieren auf Menschen ist lange bekannt. Der Anstieg meningealer Reizungen von Jelšovce-Nitra zu Jelšovce-Mad'arovce ist möglicherweise auch durch die zunehmende Übertragung von Erregern von Tieren auf den Menschen verursacht (z.B. Aufstockung der Viehbestände, s.u.). Es ist anzunehmen, dass die Menschen mit ihren Tieren unter einem Dach lebten. Neben kleinen runden Häusern sind, vor allem aus der Periode der AunjetitzKultur, bis zu 30 m lange und 8 m breite Wohnstallhäuser bekannt (HARDING 2000). Eine andere Erklärung für den Anstieg meningealer Reizungen im Verlauf der Frühbronzezeit ( 10 \% versus 20 - 30 \%) wäre das epidemische Auftreten neuer, virulenterer Erreger gegen Ende der Frühbronzezeit. Im Mittelalter hat sich die Häufigkeit meningealer Erkrankungen etwa verdoppelt (45 - 65 \% versus 10 - 30 \%) (vgl. Tab. 24 i). Noch schlechtere Ernährungslage, noch engeres Zusammenleben, u.a. mit Tieren, und schlechtere hygienische Bedingungen sind hierfür vermutlich ursächlich. 
Die hohe Belastung mit Entzündungserkrankungen in der Mad'arovce-Kultur ist auch durch die häufige Koinzidenz mit Skorbut zu erklären. Die Minderversorgung mit Vitamin C führt auch zu einer bedeutenden Beeinträchtigung des Immunsystems und daraus folgend zu einer Erhöhung der Infektanfälligkeit. Aus diesem Grund treten Entzündungserkrankungen bei den Kindern aus Jelšovce-Mad'arovce häufiger auf, als in anderen Populationen mit einer geringeren Skorbutbelastung. Es ergibt sich ein Circulus vitiosus: für Wachstum und Gesundung wird besonders viel Vitamin C benötigt. Erkrankungen wiederum können die Aufnahme von Vitamin C beeinträchtigen, so dass ein Vitamin C Mangel verschärft wird. Das im Mittelalter sehr weit verbreitete Auftreten von Skorbut lässt sich neben der schlechten Ernährungslage möglicherweise auch mit einer hohen Arbeitsbelastung der Kinder und damit einem gesteigerten Bedarf an Vitamin C erklären. Kinderarbeit war im Mittelalter schon im Alter von sechs oder sieben Jahren üblich (ARNOLD 1989). Im Gegensatz dazu treten verstärkte Muskelmarken als Zeichen starker körperlicher Belastungen bei den Kindern der Mad'arovceKultur erst mit acht bis zwölf Jahren auf.

Unterschiede im materiellen Reichtum verschiedener Populationen beeinflusst ebenfalls deren Lebensbedingungen. Die besonders reichen Populationen aus Franzhausen-I und İkiztepe weisen insgesamt weniger Erkrankungen auf als die weniger reichen Populationen aus Hainburg, Gemeinlebarn-F, Jelšovce-Nitra und Pitten. Dies scheint aber mehr für die frühe als für die späte Frühbronzezeit zu gelten, da die relativ reiche Population aus Jelšovce-Mad'arovce von den meisten Erkrankungen stärker als alle anderen bronzezeitlichen Populationen betroffen ist. Hier schein der klimatische Einfluss eine größere Rolle zu spielen (s.o.). Die Beob-achtung, dass Mittelohrerkrankungen vor allem in den unteren Bevölkerungsschichten auftreten (GRESKY et al. 2003, SCHULTZ 1989 a), lässt sich für die frühe Bronzezeit nicht machen. Franzhausen-I ist zwar relativ wenig, Gemeinlebarn-F, Hainburg und Pitten häufiger betroffen. Andererseits treten auch in den reichen Populationen von Jelšovce-Mad'arovce und 
İkiztepe relativ viele Erkrankungsfälle auf. In Jelšovce sind dabei auch besonders reiche Bestattungen betroffen.

Durch den klimatischen Wandel einerseits und die jahrhundertelange Nutzung der Böden in Jelšovce andererseits kam es zu einer Degradierung der Ackerböden mit zunehmendem Nährstoffverlust. Und damit durch die Verschlechterung der Ernährungslage zu einer Verschlechterung der Lebensbedingungen im Laufe der Frühbronzezeit. Bei gleichzeitiger Zunahme der Populationsgröße von Nitra- und Aunjetitz- zu Mad'arovce-Kultur müssen mehr Menschen von den gleichen landwirtschaftlichen Nutzflächen versorgt werden. Dies führt zu einer weiteren Verschärfung der Ernährungsprobleme. Es ist vor allem mit einem saisonal bedingten Mangel an Nahrung und Vitaminen zu rechnen.

Diesem Problem bei der Beschaffung pflanzlicher Nahrung begegneten die Menschen aus Jelšovce-Mad'arovce möglicherweise in einer verstärkten Nutzung von tierischen Nahrungsquellen. Möglicherweise haben sie ihre Viehbestände vergrößert und vermehrt Viehzucht betrieben. Zum einen steigt damit die Gefahr der Übertragung von Erkrankungen von Tieren aus den Menschen (s.o.). Zum anderen kann der vermehrte Konsum von Fleisch den Rückgang von durch Eisenmangel verursachten Anämien und damit den Rückgang von Anämien von Jelšovce-Aunjetitz zu -Mad'arovce erklären ( $n=12$ / 31: 38,7 \% versus $n=5$ / 47: 10,6 \%). Vielleicht herrschte auch zur Zeit der Aunjetitz-Kultur ein etwas feuchteres und wärmeres Klima vor als zur Zeit der Mad'arovce-Kultur, dass z.B. Wurmerkrankungen und in ihrer Folge Anämien begünstigte. Auch das endemische Auftreten einer Malaria ist als Anämieursache denkbar. Eine andere mögliche Erklärung für den Rückgang von Anämien in JelšovceMad'arovce ist im gesellschaftlichen Wandel zu suchen. Die familiären Verhältnisse werden wichtiger. Dies zeigen z.B. auch strukturelle Veränderungen in der Belegung des Gräberfeldes. Während in Jelšovce-Nitra und -Aunjetitz die Verstorbenen in Reihengräber bestattet wurden, findet man in der Mad'arovce-Kultur in Gruppen angeordnete Gräber, zum Teil um zentral gelegene Grabhügel gruppiert. Die Verstorbenen scheinen im Verband der Großfami- 
lien bestattet worden zu sein (vgl. IV.1.1). Vielleicht wurden die "eigenen Kinder" besser mit Fleisch und vitaminreicher Nahrung versorgt als in den Jahrhunderten zuvor, in denen möglicherweise vor allem der arbeitende, direkt zum Lebensunterhalt der Gemeinschaft beitragende Teil der Bevölkerung mit hochwertiger Nahrung versorgt wurde. Eine verstärkte Fürsorge gegenüber den schwächeren Mitgliedern der Gemeinschaft, die nicht unmittelbar dem Überleben der Gruppe dienen, ist wahrscheinlich. Neben ernährungsbedingten Ursachen (Eisen-, Proteinmangel) sind chronische Infektionserkrankungen (Infektanämie) oder chronischer Blutverlust zum Beispiel bei chronischen Darminfektionen (z.B. durch Parasiten) oder Skorbut als Ursachen einer Anämie denkbar. Bei einem Vitamin C Mangel kann es neben einer Anämie durch Blutverluste auch durch die Herabsetzung der enteralen Eisenresorption zu einer Anämie kommen.

Unterschiede im Auftreten von Zahnerkrankungen lassen sich ebenfalls teilweise mit mangelhafter Ernährung oder begleitenden Erkrankungen erklären.

Zur Beurteilung der Ernährungslage werden vor allem Kariesfrequenz, Abrasion und transversale Schmelzhypoplasien herangezogen (CARLI-THIELE 1996). Aufgrund fehlender Daten zur Abrasion, können hier nur Aussagen zu Karies und transversalen Schmelzhypoplasien gemacht werden.

Karies tritt in Mittelalter und Neolithikum relativ häufig auf. Zwischen 15 und $31 \%$ der Kinder aus Bettingen, Straubing und Wandersleben sind betroffen. Im Gegensatz dazu sind es in der Bronzezeit nur zwischen 4 und 14 \%, mit Ausnahme von Jelšovce-Aunjetitz mit 20\%. Dieser Unterschied weist auf Ernährungsunterschiede hin und legt Unterschiede im Zuckergehalt der Speisen nahe. Der Unterschied in der Karieshäufigkeit zwischen Jelšovce-Aunjetitz und Jelšovce-Mad'arovce lässt sich mit dem Rückgang des Konsums von kohlenhydratreicher, pflanzlicher Nahrung erklären. Vermutlich spielt jedoch auch die Härte der Zahnsubstanz und die Zusammensetzung des Speichels in diesem Zusammenhang eine Rolle. Sie sind 
interindividuell unterschiedlich und bedingt dadurch eine unterschiedliche Anfälligkeit für Karies.

Als Folge von Mangelernährung und schweren Erkrankungen kommt es zu Funktionsstörungen der zahnhartsubstanzbildenden Zellen und dadurch Minderwertigkeit von Schmelz und Dentin (s. III.3.5). Dies äußert sich in transversalen Schmelzhypoplasien.

Die transversalen Schmelzhypoplasien wurden bei den Mad'arovce-Kindern bereits mit anderthalb bis zwei Jahren gebildet. Das gleiche stellte Schultz (SCHULTZ 1988/89) bei der Untersuchung der Hainburger Kinder fest. Er schließt aus dem frühen Auftreten von transversalen Schmelzhypoplasien, dass die Kinder nicht über das zweite Jahr hinaus gestillt wurden. Das Gleiche ist für die Kinder der Mad'arovce-Kultur anzunehmen. In Gegensatz dazu sind die transversalen Schmelzhypoplasien der Kinder aus İkiztepe später entstanden (SCHULTZ 1990 und 1988/89). Eine längere Stillzeit für die Kinder aus İkiztepe lässt auch das geringe Auftreten von Karies vermuten (s.o.). Längeres Stillen schützt die Zähne vor Karies (SCHULTZ 1990). Die vergleichsweise niedrige Kariesfrequenz bei den Kindern der Mad'arovce-Kultur aus Jelšovce steht nicht im Zusammenhang mit der Länge der Stillzeit. Viel wahrscheinlicher spiegelt sie eine mangelhafte, kohlenhydratarme Ernährung wider (s.o.).

Die Entwöhnung der Kinder vom Stillen ist ein besonders riskanter Lebensabschnitt (meist zwischen 6. und 24. Monat). Die Umstellung von flüssiger aus breiförmige und feste Nahrung beinhaltet insbesondere die Gefahr der Mangelernährung. Der fehlende, in der Muttermilch enthaltene Immunschutz, begünstigt die Entstehung von schweren gastrointestinalen Infekten. Die Muttermilch steigert die Widerstandsfähigkeit des Kindes gegen Infektionen und schützt es vor Über-, Unter- und Fehlernährung. Vergleichsweise relativ niedrige Sterblichkeit bei unter 2-jährigen und "gute" Zähne sowie wenige transversalen Schmelzhypoplasien in ganz jungen Jahren weisen auf eine relativ lange Stillzeit hin. Unterschiede in der Mortalität der unter 2-jährigen in Jelšovce legen nahe, dass in Nitra- und Aunjetitz-Kultur länger als in Mad'arovce-Kultur gestillt wurde. 
Das häufige Auftreten von transversale Schmelzhypoplasien (56 - 63 \%) in JelšovceMad'arovce und -Aunjetitz, Franzhausen-I und Wandersleben zeigt, dass viele der Kinder dieser Populationen erheblichem Stress (z.B. Mangelernährung, Erkrankungen) ausgesetzt waren. Es heißt aber auch, dass etliche diesen Stress überlebt haben, sonst wären die transversalen Schmelzhypoplasien für uns heute nicht sichtbar. In Populationen mit weniger transversalen Schmelzhypoplasien haben viele Kinder vielleicht die Beeinträchtigungen überhaupt nicht überlebt, so dass sich keine später sichtbaren transversalen Schmelzhypoplasien entwickeln konnten („osteological paradox“, WoOD et al. 1992).

In Bezug auf Parodontopathien scheint weniger die Ernährung als vielmehr das unterschiedlich häufige Auftreten von begleitenden Erkrankungen eine Rolle zu spielen. Zum Beispiel kann ein Vitamin C Mangel zur Entstehung von Parodontopathien beitragen. Auf dem Boden (chronischer) Blutungen und geschwächter Immunabwehr kann es zu Entzündungen des Zahnfleisches kommen. Das relativ häufige Auftreten von Skorbut in Jelšovce-Aunjetitz und Mad'arovce passt zu dem ebenfalls häufigen Auftreten von Parodontopathien (vgl. IV.3). Insgesamt zeigt sich, dass Parodontopathien häufiger als Skorbut auftreten (Tab. 20). Je häufiger Skorbut in einer Population auftritt, desto häufiger sind auch Parodontopathien. Nur Hainburg bildet hier eine Ausnahme. Trotz einer Belastung mit Parodontopathien von 75 \%, konnte kein einziger Fall von Skorbut festgestellt werden. Auch in Jelšovce zeigt sich, dass eine hohe Skorbutbelastung nicht als alleinige Ursache für einen starken Befall mit Parodontopathien angesehen werden kann. In Jelšovce-Mad'arovce sind im Vergleich zu Jelšovce-Aunjetitz bei entsprechend starker Skorbutbelastung ( $n=12$ / 49: 24,5 bzw. $n=10$ / 43: 23,3 \%) Parodontopathien häufiger ( $\mathrm{n}=26 / 41:$ 63,4 \% bzw. $\mathrm{n}=11 / 24:$ 45,8 \%). Zum Beispiel trägt auch ein starker Zahnsteinbefall zur Entstehung von Parodontopathien bei (s. III.3.2). Es zeigt sich, dass die Kinder aus Jelšovce-Mad'arovce von diesem stärker betroffen sind als die Kinder von Jelšovce-Aunjetitz. 
Da nur Einzelfälle von Rachitis auftraten, ist davon auszugehen, dass die Kinder aller Kulturen dem Sonnenlicht ausreichend exponiert waren. Als Ursachen für die dennoch aufgetretenen Rachitisfälle werden vor allem längere Bettlägerigkeit durch schwere Erkrankungen in dunklen Behausungen angeschuldigt. Einzig bei den Kindern der AunjetitzKultur in Jelšovce sind aufgrund des etwas gehäufteren Auftretens auch genetische bedingte Fälle von Rachitis, z.B. bei erblichen Nierenerkrankungen denkbar.

Zusammenfassend lässt sich sagen, dass die Ursachen für die beobachteten Unterschiede in den unterschiedlichen Biotopen, sowie in den Änderungen der klimatischen Bedingungen im Verlauf der Frühbronzezeit zu suchen sind. Sich wandelnde kulturelle und gesellschaftliche Grundlagen scheinen ebenfalls eine Rolle zu spielen. Auch die Größe der Populationen und deren Reichtum beeinflussten die Lebensbedingungen der Menschen.

\section{IV.8 Fallbeispiele}

IV.8.1 Fallbeispiel 1: Skorbut und entzündliche Erkrankungen der pneumatischen Räume des Schädels

Bei dem Kind aus Grab 65 A handelt es sich um ein drei bis vier Jahre altes Individuum unbestimmbaren Geschlechts, das sowohl an Skorbut als auch an verschiedenen entzündlichen Erkrankungen litt. In Einzelnen konnten folgende Diagnosen gestellt werden:

$\begin{array}{ll}\text { meningeale Reizung } & \text { Zahnstein } \\ \text { Hirndrucksymptomatik } & \text { transversale Schmelzhypoplasien } \\ \text { Rhinitis } & \text { Harris-Linien } \\ \text { Sinusitis maxillaris } & \text { Skorbut } \\ \text { Otitis media } & \end{array}$


Transversale Schmelzhypoplasien und Harris-Linien zeigen, dass es zu verschiedenen Wachstumsstillständen gekommen ist, die entweder durch länger andauernde Krankheit oder Hungerperioden, wahrscheinlich einer Kombination aus beidem, verursacht wurden (Abb.33). Als Zeichen einer Mangelernährung hat sich ein Skorbut manifestiert. Unterschiedlich alte Blutungszeichen an verschiedenen Stellen (s. Katalog) zeigen den typischen subförmigen Verlauf der Erkrankung mit rezidivierend auftretenden Blutungen. Fast alle typischen Regionen sind betroffen (vgl. III.5.1.1) (Tafeln 21, 23, 49 und 50). Mangelernährung und Skorbut führten zu einer allgemeinen Schwächung des Immunsystems und damit zu einer erhöhten Anfälligkeit für entzündliche Erkrankungen (Abb. 33). Diese äußert sich in dem Auftreten von Erkrankungen des Nasen-Rachenraumes. Die Infektion von Kieferhöhlen und Mittelohr führte, begünstigt durch die schlechte Abwehrlage des Kindes, zu einer chronischen Entzündung. In der Folge kam es zum Auftreten von Komplikationen (Abb. 33). Meningeale Veränderungen traten entweder durch Ausbreitung von Krankheitserregern aus dem Nasenrachenraum oder als Folge von Einblutungen im Rahmen des Skorbutes auf. Möglicherweise verstarb das Kind in diesem Zusammenhang an einer Sepsis oder an dem Anstieg des intrazerebralen Drucks (Abb. 33).

\section{IV.8.2 Fallbeispiel 2: Skorbut und Anämie}

Bei dem Kind aus Grab 72 handelt es sich um ein eineinhalb bis zwei Jahre altes Individuum unbestimmbaren Geschlechts mit sowohl entzündlichen als auch durch Mangelernährung verursachten Erkrankungen. Folgende Diagnosen konnten erhoben werden:

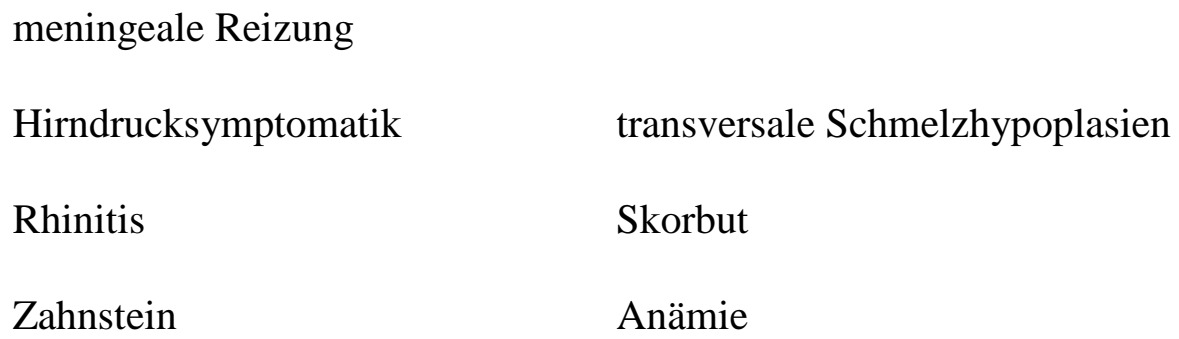


Das Kind litt sowohl an Anämie als auch an Skorbut (Tafeln 12, 13 und 49). Die Anämie ist möglicherweise als Folge des Skorbuts entstanden (Abb. 34). Zum einen führen rezidivierende Blutungen zu einer chronischen Blutungsanämie, zu anderen führt der Vitamin C Mangel zu einer Eisenresorptionsstörung und damit zu einem Eisenmangel. Auch ein ernährungsbedingter Proteinmangel ist als Ursache für die Anämie möglich. Wahrscheinlich haben alle diese Faktoren eine Rolle gespielt. Auch dieses Kind litt an einer Schwächung des Immunsystems und war damit besonders anfällig für Infekte (Abb. 34). Das Auftreten von transversalen Schmelzhypoplasien bei einem so kleinen Kind, spricht dafür, dass es bereits länger nicht mehr gestillt wurde. Die Skorbuterkrankung weist in dieselbe Richtung. Die mikroskopische Untersuchung der rechten Ulna hat gezeigt, dass diese eine Inaktivitätsatrophie aufweist. Das Kind scheint schon längere Zeit bettlägerig gewesen zu sein (Abb. 34). Auch bei diesem Kind ist nicht $\mathrm{zu}$ sagen, ob die meningealen Veränderungen entzündlicher oder hämorrhagischer Natur sind (s. IV.8.1).

\section{IV.8.3 Fallbeispiel 3: Tumorerkrankung}

Bei dem Kind aus Grab 616 B handelt es sich um ein neun bis elf Jahre altes weibliches Individuum. Folgende Diagnosen konnten erhoben werden:

$\begin{array}{lc}\text { Sinusitis maxillaris } & \text { transversale Schmelzhypoplasien } \\ \text { Karies } & \text { V.a.. ossären Tumor der linken Tibia } \\ \text { Parodontose } & \text { sternale Epiphysendysplasien beider } \\ \text { Zahnstein } & \text { Clavicula } \\ \text { Harris-Linien } & \end{array}$

Wie transversale Schmelzhypoplasien und Harris-Linien zeigen, litt auch dieses Kind in früher Kindheit unter Mangelernährung oder Erkrankungen (Abb. 35). Außerdem zeigt es wahrscheinlich genetische bedingte Dysplasien der sternalen Claviculaepiphysen (s. III.4.1.1). Interessant ist die tumoröse Veränderung der linken proximalen Tibia (s. Katalog) (Tafeln 40 
und 41). Höchstwahrscheinlich handelt es sich um einen primären Knochentumor, der die gesamte Struktur der Tibia verändert hat. Im Kindesalter gibt es zahlreiche Tumoren die sich am Knochen, bevorzugt in der Kniegelenksregion, manifestieren. Differentialdiagnostisch kommen bei diesem Kind vor allen ein Osteosarkom, ein Osteoblastom oder ein EwingTumor in Frage. Beide führen zu atypischen Knochenneubildungen.

Ein Osteosarkom tritt in 60\% aller Fälle am distalen Femur oder an der proximalen Tibia auf. Es geht in der Regel von der Metaphyse aus und hat neben fibrösen Anteilen immer eine osteoblastische Komponente. In 50 \% der Fälle sind Kinder zwischen dem 10. und dem 20. Lebensjahr betroffen. Histologisch werden verschiedene unterschiedlich schnell wachsende Typen unterschieden. Neben einem osteolytischen gibt es zum Beispiel den sklerotischen Typ, dabei können fibro-, chondro-, oder osteoblastische Anteile überwiegen (HUVOS 1979). Das Osteosarkom infiltriert das Knochenmark, es kann zu sogenannten „Tumornestern“ kommen (MIRRA 1980), im Gegensatz zum Osteoblastom sind diese von der Umgebung nicht scharf abzugrenzen. Häufig führt der Tumor zu einer Arrosion der Corticalis mit begleitender Periostreaktion. Es kommt zu einer Neubildung von atypischem primärem Faserknochen (JunDT 2001, KLINGEBIEL 2001). Vor allem der sklerotische Typ ist leicht mit einem Osteoblastom zu verwechseln (MIRRA 1980).

Osteoblastome treten ebenfalls meist im Alter zwischen zehn und 20 Jahren auf und befallen vor allem Dia- und Metaphysen. Die Knieregion ist dabei selten betroffen. Meist tritt eine begleitende Periostreaktion auf. Zu einer Infiltration des Knochenmarks kommt es in der Regel nicht (MIRRA 1980). Im Unterschied zum Osteosarkom kommt normale neben pathologischer Spongiosa vor, wobei diese scharf voneinander getrennt sind (MIRRA 1980).

Ewing-Tumoren treten besonders an den langen Röhrenknochen, aber auch an Becken und Schulterblättern auf. Diese Tumoren gehen typischerweise von den Diaphysen aus und zeigen röntgenologisch ein mottenfraßartiges Bild mit Osteolysen, Knochenneubildungen besonders auch im Bereich des Periosts (JundT 2001, KlingeBIEL 2001, Huvos 1979). Meist kommt 
es zu einer massiven Deformierung des Knochens (STEINBOCK 1976) Auch hier kann eine Arrosion der Corticalis auf treten. Andere Knochentumoren wie z.B. Chondroblastome, Chondrosarkome oder Osteochondrome zeichnen sich durch Osteolysen oder begrenzte Exostosen aus und kommen daher als Ursache für die Veränderungen bei diesem Kind nicht in Frage (JUNDT 2001, JANI \& KRÄMER 2001).

Bei dem Kind aus Grab 616 B scheint der Tumor von der Metaphyse der proximalen Tibia ausgegangen zu sein. Er hat sowohl Epi- als auch Diaphyse infiltriert und zu einer vorzeitigen atypischen Verknöcherung der beiden Metaphysenplatten im zentralen Bereich geführt (Tafel 40). Er weist Anzeichen eines destruierenden Wachstums mit starker osteoblastischer Aktivität auf. Die Corticalis ist ausgedünnt, der Knochen deformiert und die normale Spongiosa durch feine schnellgewachsene, atypisch konfigurierte Spongiosa ersetzt (Tafeln 40 und 41). Normale und pathologische Spongiosa sind dabei nicht klar voneinander zu trennen.

Aufgrund der Lokalisation und des histologischen Befundes handelt es sich am ehesten um ein Osteosarkom vom sklerotischen Typ. Dieser scheint nicht sehr aggressiv gewachsen zu sein, da Merkmale eines überstürzten Knochenab- und -umbaus fehlen.

Eine Metastasierung des Tumors ist nicht nachweisbar, aber bei der gefundenen Tumorgröße durchaus denkbar. Da Lungen- oder Lebermetastasen primär keine Veränderungen an den Knochen hinterlassen, ist es durchaus möglich, dass diese bestanden haben, aber für uns nicht mehr erkennbar sind.

Da Tumorerkrankungen durch vom Tumor induzierte Immunsuppression zu einer Schwächung des Immunsystems führen, ist es denkbar, dass die Infektion des Sinus maxillares durch diese begünstigt wurde (Abb. 35).

IV.8.4 Fallbeispiel 4: perimortales Schädeltrauma

Bei dem Kind aus Grab 253 handelt es sich um ein zwölf bis 18 Jahre altes weibliches Individuum. Bis auf massive Verletzungen des knöchernen Schädels war diese junge Frau gesund. 
Sie weist kombinierte Verletzungen des Schädeldachs, der Schädelbasis und des Jochbogens auf. Es muss von einem Schädelhirntrauma mit schweren intrazerebralen Blutungen ausgegangen werden (Abb. 36). Fehlende Heilungsspuren zeigen, dass die Verletzungen tödlich waren. Neben einer Sprengung der Sutura zygomaticomaxillaris rechts mit Kantenabsprengung am Os zygomaticum, besteht auf beiden Seiten des Foramen magnum eine Fraktur des Os occipitale mit Absprengung von Clivus und Condylen. Zudem weist das Os temporale rechts einen Lochdefekt mit davon ausgehenden radiären Sprüngen der Schädelkalotte auf (Tafeln 4 und 5) der höchstwahrscheinlich durch einen gezielten Schlag auf den Kopf verursacht wurde (Abb. 36).

\section{IV.8.5 Fallbeispiel 5: Skorbut, meningeale Reizung und Kauterisation}

Bei dem Kind aus Grab 228 B handelt es sich um ein sechs bis acht Jahre altes, weibliches Individuum. Folgende Diagnosen konnten erhoben werden:

$\begin{array}{ll}\text { meningeale Reizung } & \text { Zahnstein } \\ \text { Perisinusitis } & \text { transversale Schmelzhypoplasien } \\ \text { Hirndrucksymptomatik } & \text { verstärkte Muskelmarken } \\ \text { Parodontose } & \text { Skorbut } \\ \text { V.a. Kauterisation } & \end{array}$

Stark ausgeprägte Muskelmarken weisen auf eine außergewöhnliche Beanspruchung der Muskel hin ("Kinderarbeit"). Außerdem litt das Mädchen an Skorbut. Die bestehende Mangelernährung wurde durch schwere Arbeit möglicherweise noch verstärkt, da bei körperlicher Anstrengung der Bedarf an Nährstoffen gesteigert ist (Abb. 37). Ein Vitamin C Mangel beeinträchtigt bekanntlich das Immunsystem und führt zu einer gesteigerten Infektanfälligkeit (Abb. 37). Ob die meningealen Veränderungen entzündlich bedingt oder hämorrhagischen Ursprungs sind, ist allerdings nicht mehr festzustellen. In jedem Fall hat das Kind an einer Erhöhung des intracraniellen Druckes mit begleitender Hirndrucksymptomatik gelitten. 
Eine besondere Veränderung zeigt das Os frontale. Mittig etwas unterhalb der Sutura coronaria befindet sich eine gut vernarbte, muldenartige, fast runde Vertiefung der Lamina externa. Entzündliche Begleitveränderungen finden sich nicht (Tafel 5).

Differentialdiagnostisch kommen verschiedene Ursachen in Frage. Eine tangentiale Schwerthieb oder eine Impressionsfraktur z.B. durch einen Hammerschlag auf die Stirn sind denkbar, ebenso ein tumoröses Geschehen oder eine durch Druck verursachte Atrophie des Knochens. Wahrscheinlich handelt es sich jedoch um das Zeichen eines medizinischen Behandlungsversuches in der Bronzezeit. Die Veränderung könnte Zeichen einer Kauterisation, einer Ausbrennung der Kopfschwarte, sein, die bis auf das Schädeldach reicht. Aufgrund von Lokalisation und Erscheinungsbild, die Läsion ist mittig, frontal und sehr regelmäßig, beinahe rund, ist die Veränderung eher nicht durch Verletzung oder nicht regelhaftes Wachstum verursacht. Diese würden ein weniger gleichmäßiges Erscheinungsbild erwarten lassen. Auch Alter und Geschlecht des Kindes sprechen eher gegen eine Schwert- oder Hammerverletzung. Obwohl diese z.B. bei einem Überfall auf das Dorf natürlich denkbar wäre. Das Ausführen von Kauterisationen zur Zeit der Mad'arovce-Kultur in Jelšovce ist durchaus denkbar, da die Chirurgie der Trepanation ebenfalls praktiziert wurde.

Die Kauterisation als Mittel zur Behandlung von Kranken ist schon sehr lange bekannt. Schon in vorschriftlichen Kulturen finden sich Zeichen von Ausbrennungen an Knochen. Schriftliche Erwähnungen sind aus Mesopotamien und dem Ägypten des 3. Jh. v.Chr. bekannt (LYONS \& PetRUCELLI 1980). In Ägypten wurde die Kauterisation zur Entfernung von oberflächlichen Tumoren oder Zysten eingesetzt (LYONS \& PETRUCELLI 1980). Auch zur Blutstillung wurde die Methode der Kauterisation angewandt. Auch im antiken Griechenland und im römischen Reich war das Kauterisieren üblich (RÜSTER 1993, LYONS \& PETRUCELLI 1980, SuDHOFF 1914). Das Hippokrates zugeschriebene Zitat : "Was das Medikament nicht heilt, heilt das Messer; was das Messer nicht heilt, heilt das Feuer; was das Feuer jedoch nicht heilt, 
ist unheilbar.", ist aber vermutlich schon viel älter (LYONS \& PETRUCELLI 1980). Neben der Wundbehandlung und Blutstillung bei Verletzungen oder operativen Eingriffen fand die Kauterisation auch Anwendung bei der Behandlung innerer Erkrankungen. Im Mittelalter ersetzt das Kauterisieren die meisten anderen operativen Techniken. Die Kauterisation war auch die am weitesten verbreitete chirurgische Technik des arabischen Arztes (LYONS \& PETRUCELLI 1980). Von verschiedenen Quellen werden Brenneisen als Universalmittel gegen Erkrankungen wie Epilepsie, Kopf- oder Zahnschmerzen, Hämorrhoiden, Rippenfellentzündung, Wassersucht, Melancholie und viele weitere Erkrankungen beschrieben (MURRAY JONES 1999, SCUltetus 1988, Heister 1983, LyONS \& PetruCELli 1980, Sudhoff 1914, BenNiON 1980). Außerdem kam sie als Bestrafung für bestimmte Delikte, im Rahmen religiöser Handlungen, als Zeichen der Trauer oder als Markierung von Sklaven zur Anwendung. In vielen mittelalterlichen Schriften finden sich Figuren mit markierten Kauterisationspunkten. Geeignete Punkte verteilen sich über den gesamten Körper. Auch am Kopf sind zahlreiche Punkte zu finden (Lyons \& PetruCelli 1980, Sudhoff 1914, Scultetus 1988, Heister 1983). Man hoffte, mit Kauterisation am Kopf speziell Epilepsien, starken Kopfschmerzen sowie psychischen Erkrankungen zu kurieren und böse Geister und Dämonen aus dem Kopf zu vertreiben. Aus demselben Grund wurden bei diesen Personen auch Trepanationen durchgeführt (LYONS \& PETRUCELLI 1980).

Bei dem Grab 228 B handelt es sich um eines der reichsten Kindergräber des Gräberfeldes von Jelšovce. Vielleicht entstammte das Mädchen einer reichen, bedeutenden Familie. Möglicherweise litt das Kind an starken Kopfschmerzen, Wesensveränderungen oder epileptischen Anfällen (Abb. 37). Eine Epilepsie wurde früher vielerorts als heilige Krankheit angesehen. Vielleicht zeigt sich aufgrund dessen in der überaus reichen Bestattung eines relativ jungen Individuums die besondere Verehrung zu Lebzeiten. Die gefundenen Spuren meningealer Reizungen sind Zeichen einer endocranialen Erkrankung, die durchaus starke Kopfschmerzen 
erklären können. Interessant ist auch, dass sich im Bereich der Läsion zwei Akupunkturpunkte befinden (Du-21 und Du-22), die in der traditionellen chinesischen Medizin unter anderem zur Behandlung von kindlichen Krampfanfällen, Kopfschmerzen und Bewusstlosigkeit verwendet werden (HEMPEN 1998). Letztendlich ist zum gegenwärtigen Zeitpunkt nicht sicher zu sagen, ob es sich wirklich um das Zeichen einer Kauterisation handelt und wenn ja, aus welchem Grund diese durchgeführt wurde. 


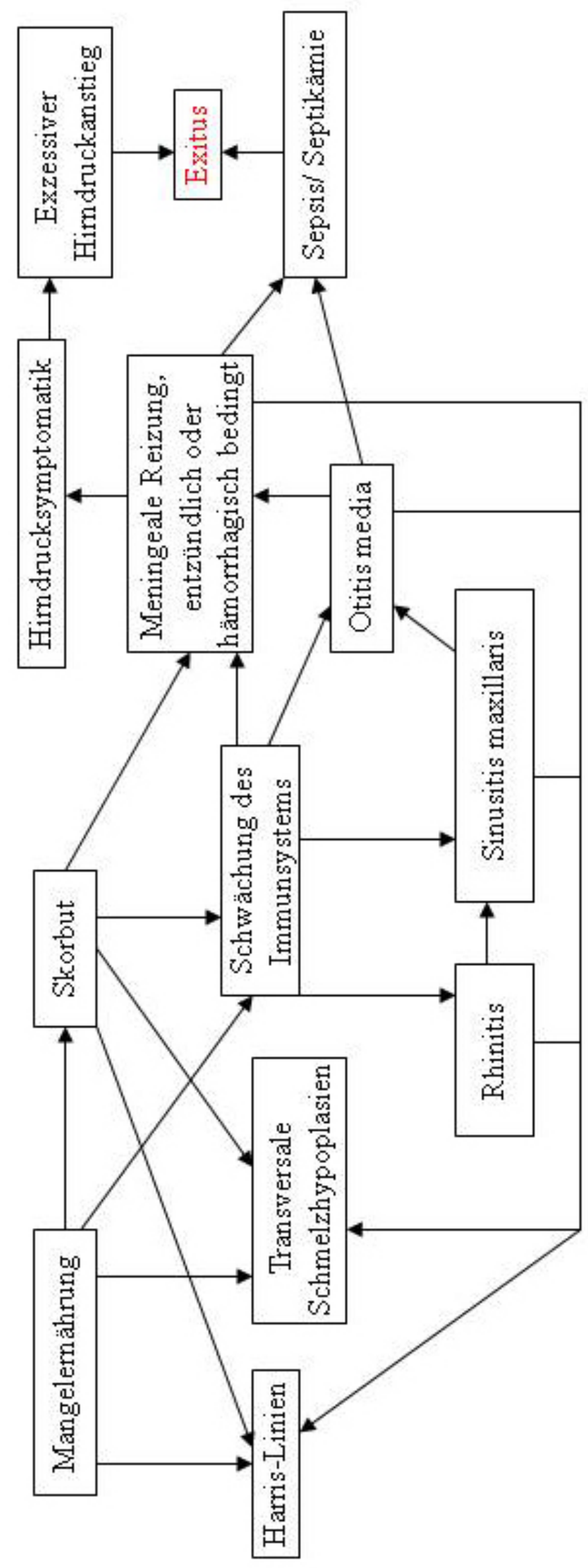




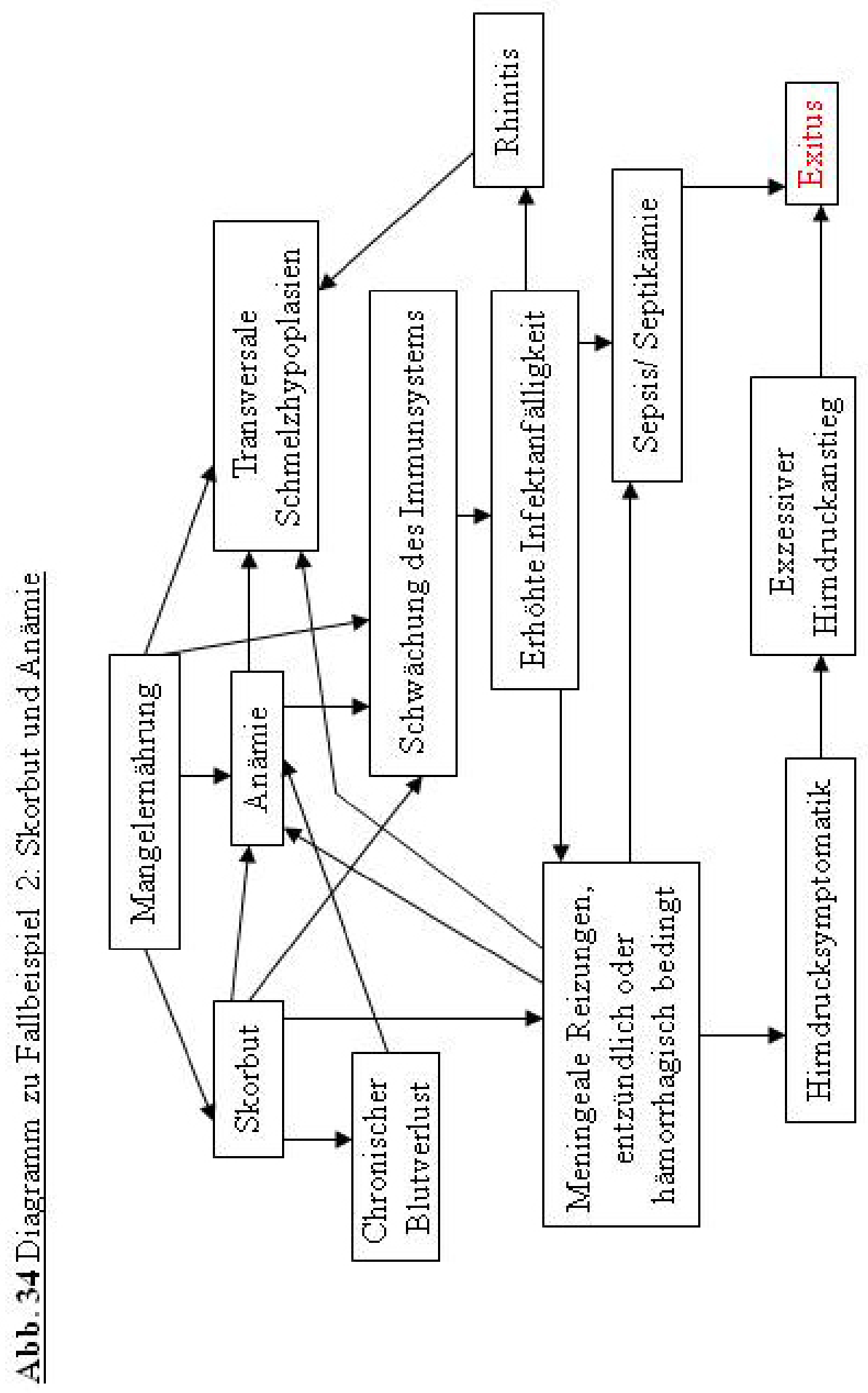




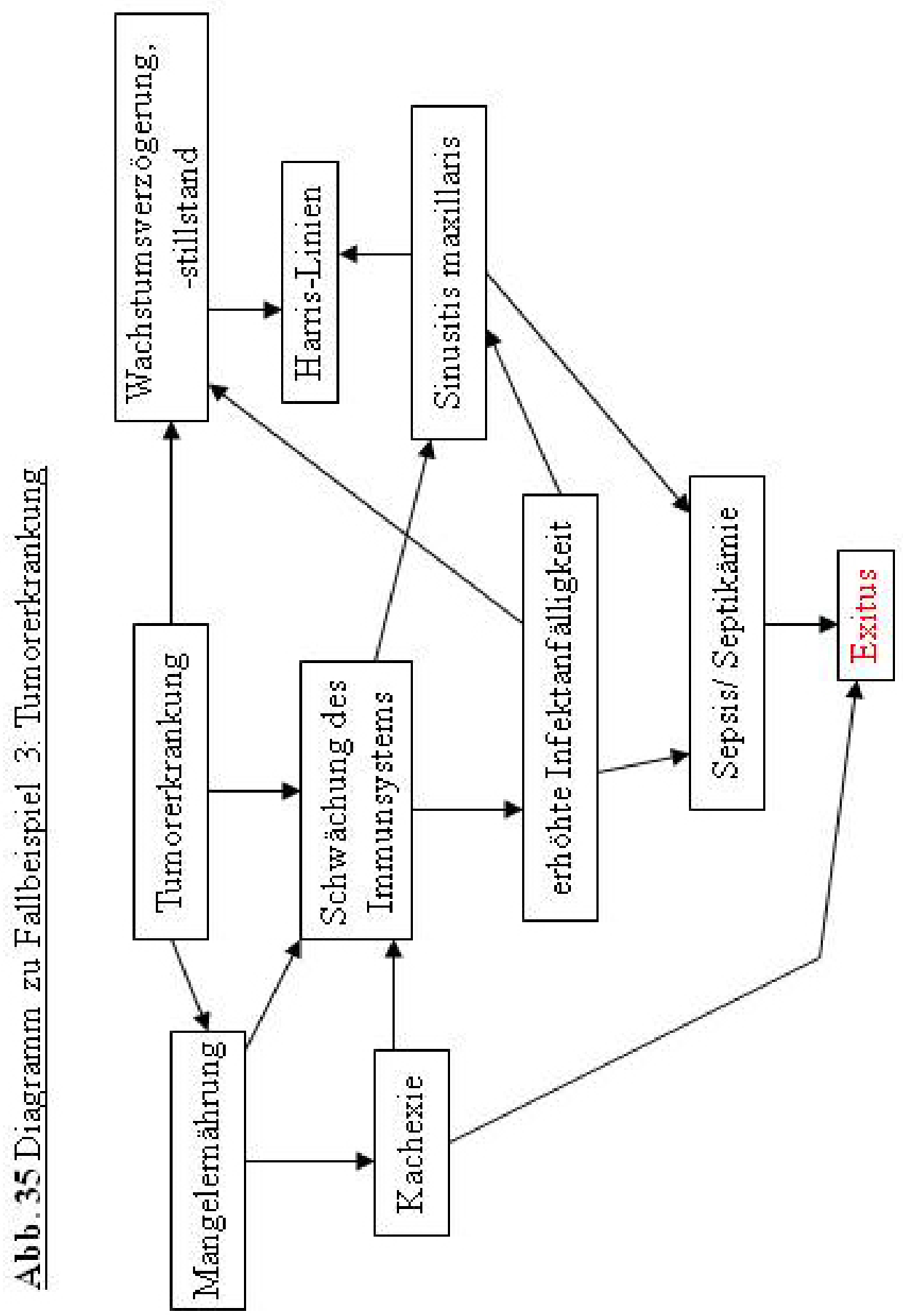




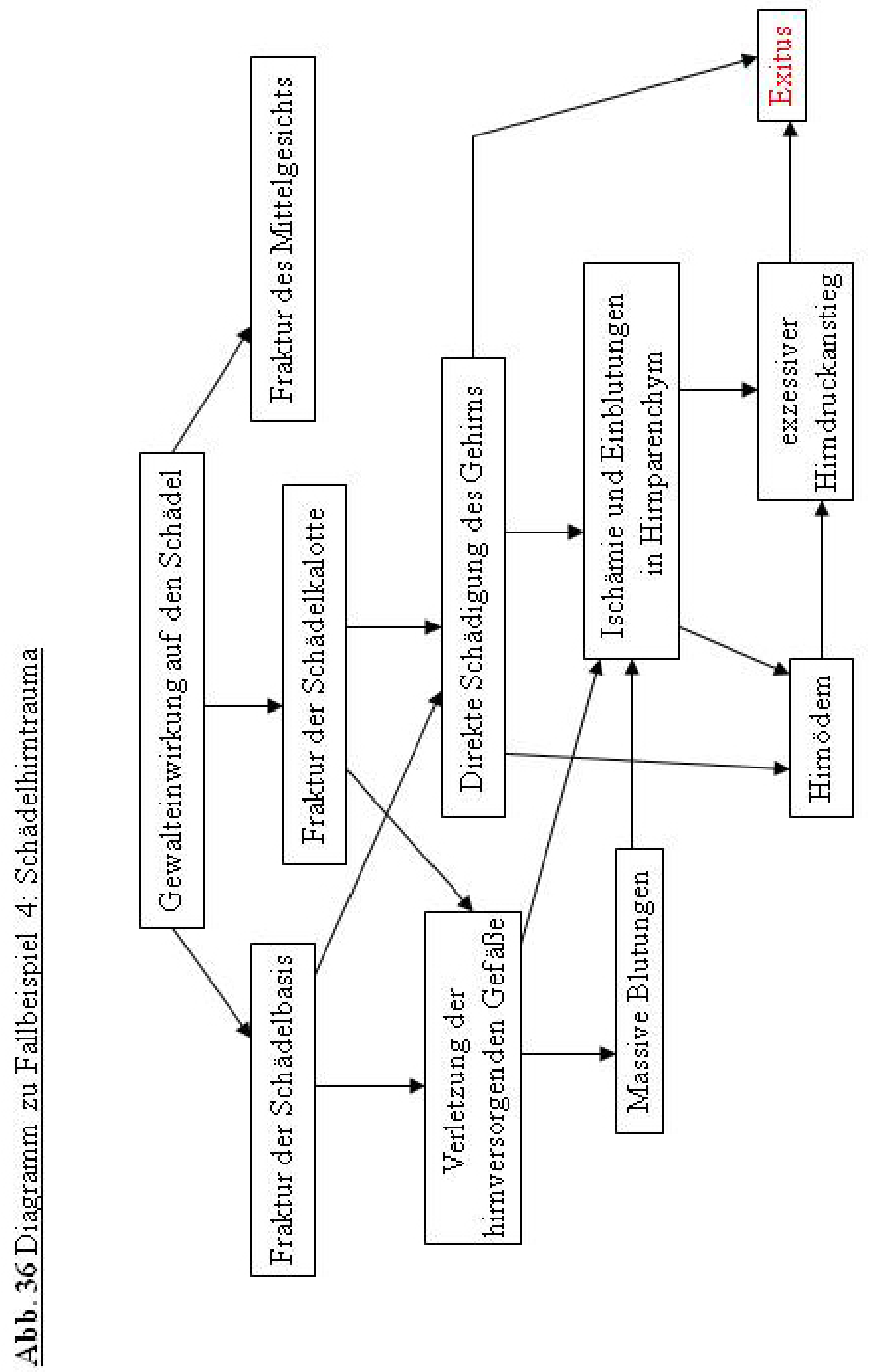




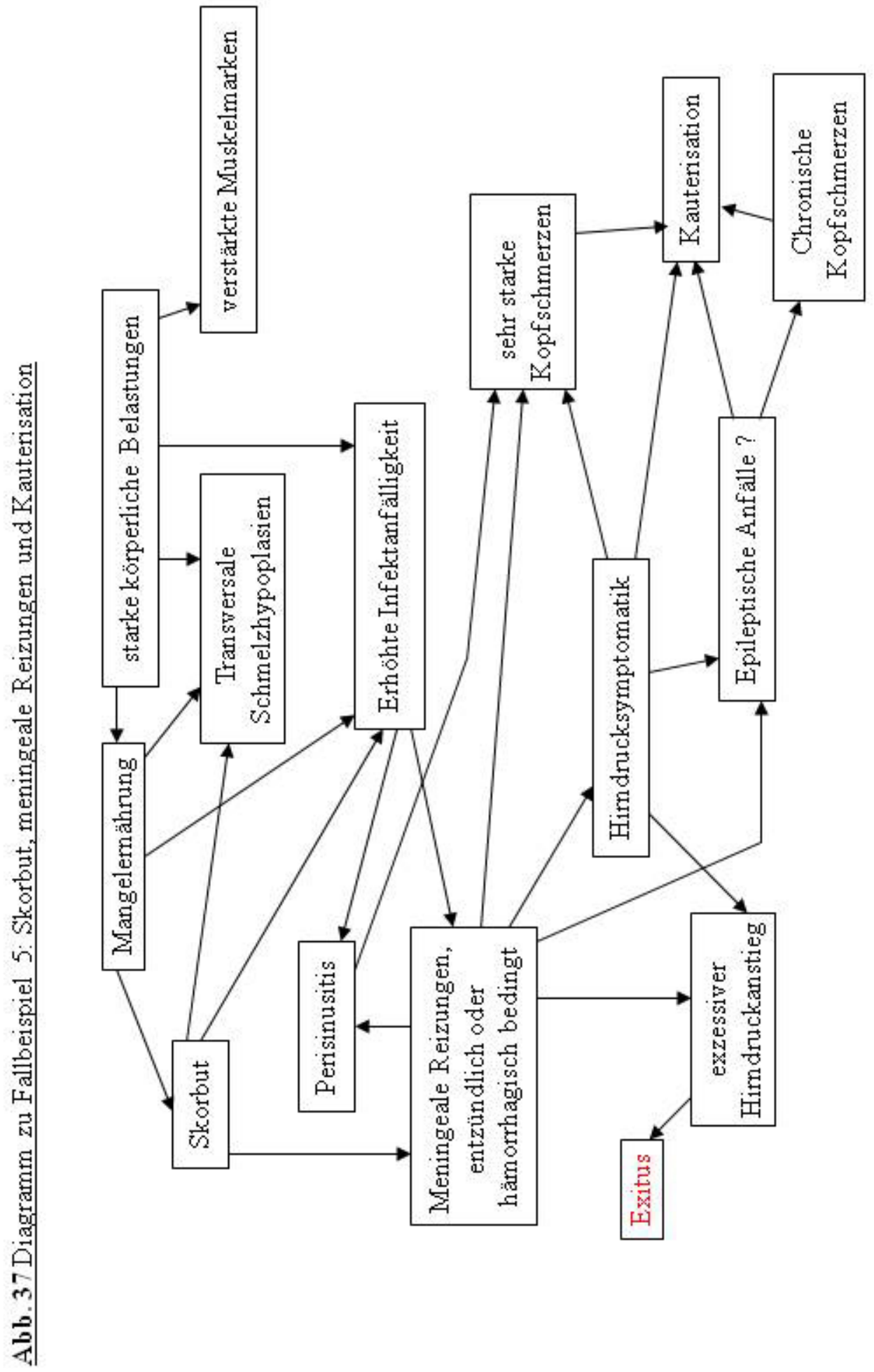




\section{$\underline{\text { V. Zusammenfassung }}$}

Im Rahmen dieser Arbeit wurden die Kinderskelete der frühbronzezeitlichen Mad'arovceKultur aus Jelšovce / Slowakei auf Erkrankungen untersucht. Die Ergebnisse wurden mit denen aus demselben Gräberfeld stammenden Kinderskeleten der Nitra- und Aunjetitz-Kultur verglichen. Außerdem wurden zu Vergleichszwecken bronzezeitliche Populationen aus dem niederösterreichischen Traisental und aus Anatolien sowie mittelalterliche und neolithische Kinderpopulationen herangezogen, die von anderen Autoren mit denselben Methoden untersucht worden waren.

Von Nitra- über Aunjetitz- zu Mad'arovce-Kultur ist ein Anstieg der Erkrankungshäufigkeiten bei den Entzündungserkrankungen zu verzeichnen. Für die Mangelerkrankungen gilt entsprechendes nur in Bezug auf die Zusammenschau aller Mangelerkrankungen. Im Einzelnen treten Anämie und Rachitis in der Aunjetitz-Kultur mit der größten Häufigkeit auf, gefolgt von Nitra- und Mad'arovce-Kultur. Von Skorbut sind die Kinder der Mad'arovce- und AunjetitzKultur gleichhäufig betroffen, die der Nitra-Kultur weniger. Bei den Zahnerkrankungen ist, mit Ausnahme von Karies, wiederum ein Anstieg der Krankheitshäufigkeiten von Nitra- über Aunjetitz- zu Mad'arovce-Kultur zu beobachten.

Die diachrone Untersuchung der frühbronzezeitlichen Kinderpopulationen von Jelšovce zeigt, dass das Krankheitsspektrum bei den Angehörigen der Nitra-, Aunjetitz- und Mad'arovceKultur annähernd gleich ist. Dies spricht für ein sehr ähnliches Biotop über den gesamten frühbronzezeitlichen Siedlungszeitraum hinweg. Für die dort lebenden Menschen waren die gleichen positiven wie negativen Aspekte relevant, was sich in der annähernden Übereinstimmung des Krankheitsspektrums zeigt. Der beobachtete Unterschied in den Krankheitshäufigkeiten wurde sehr wahrscheinlich durch eine Erschöpfung der Ressourcen sowie die Zunahme der Populationsgröße verursacht. 
In der Bronzezeit treten bei Kindern entzündliche Erkrankungen des Schädels verglichen mit mittelalterlichen Populationen eher selten auf. Im Laufe der Frühbronzezeit nimmt die Erkrankungshäufigkeit fast aller Krankheiten zu. Mit Ausnahme der Mastoiditis sind die Kinder der Mad'arovce-Kultur überdurchschnittlich häufig betroffen. Mangelerkrankungen treten mit unterschiedlicher Häufigkeit in allen drei Teilpopulationen von Jelšovce auf, sind insgesamt aber eher selten. Häufiger als diese sind unspezifische Stressmarker wie transversale Schmelzhypoplasien, Harris-Linien und unspezifische Cribra orbitalia zu beobachten. Ursachen für die beobachteten Unterschiede sind in den unterschiedlichen Biotopen sowie den sich im Verlauf der Frühbronzezeit deutlich ändernden klimatischen Bedingungen zu suchen. Sich wandelnde kulturelle und gesellschaftliche Grundlagen spielen offenbar ebenfalls eine Rolle. Auch die Größe der Populationen und deren materieller Reichtum und die damit verbundenen notwendigen Ressourcen beeinflussten die Lebensbedingungen der bronzezeitlichen Menschen.

Spuren einer Kauterisation an einem Kinderschädel weisen auf einen Behandlungsversuch im frühbronzezeitlichen Jelšovce hin. In einem anderen Fall wurde ein ossärer Tumor nachgewiesen. Bei zwei Kindern konnten Erkrankungen der Knochen festgestellt werden, die es in dieser Form heute offenbar nicht mehr gibt. Somit liefert die Untersuchung der Kinderskelete von Jelšovce auch medizinhistorisch interessante Befunde. 


\section{Anhang}

\section{Gradeinteilung der Cribra orbitalia (SCHULTZ 1988 a):}

Grad I: einzelne, feinporöse Oberflächenveränderungen

Grad II: deutlich ausgeprägte, miteinander in Verbindung tretende Lochdefekte, flache Vorwölbung der porösen Knochenneubildungen aus der Orbita

Grad III: trabekuläres Wachstum aus der Diploë des Orbitadaches in die Augenhöhle

\section{Gradeinteilung der Zahnkaries (SCHULTZ 1988 a):}

Grad I: $\quad$ stecknadelkopfgroß

Grad II: sesamkorngroß

Grad III: $\quad$ pfefferkorngroß

Grad IV: reiskorngroß

nur für Molaren gültig: $\quad$ Grad V: $\quad$ Krone zur Hälfte zerstört

Grad VI: Krone vollständig zerstört

Gradeinteilung der Transversalen Schmelzhypoplasien (SCHULTZ 1988 a, S. 494)

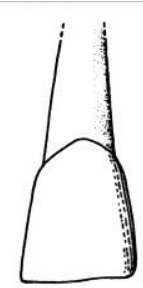

0

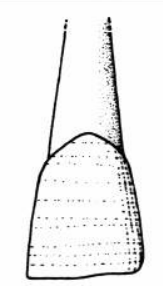

I

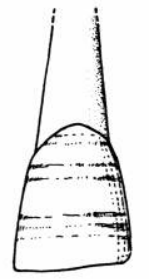

II

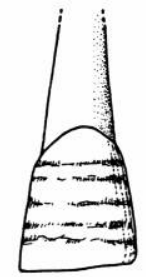

III

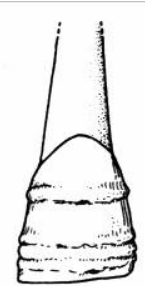

IV

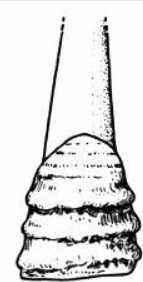

V

Grad 0: normal, Grad I: sehr schwach, Grad II: schwach, Grad II: mittel, Grad IV: stark, Grad V: sehr stark 

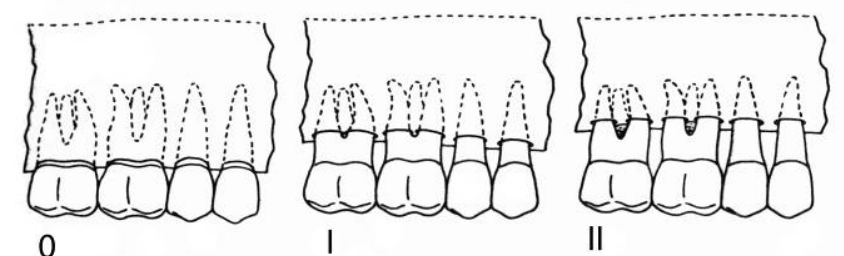

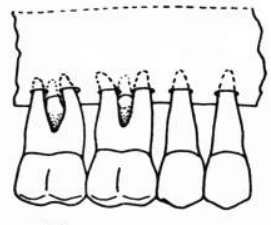

III

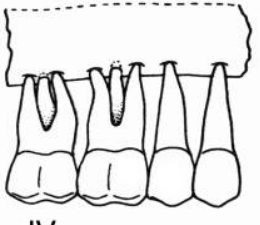

IV

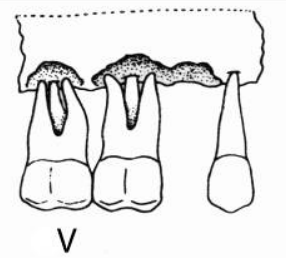

(fortschreitende Entwicklung von Parodontose bzw. Parodontitis)

Grad 0: normal, Grad I: leicht, Grad II: mäßig stark, Grad III: mittel stark, Grad IV: stark, Grad V: sehr stark

Gradeinteilung des Zahnsteins (SCHULTZ 1988 a, S. 493)
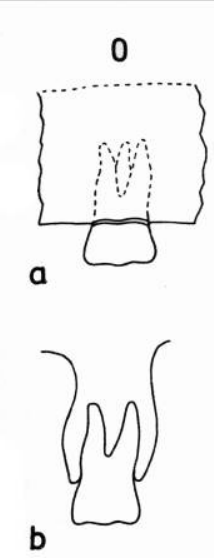
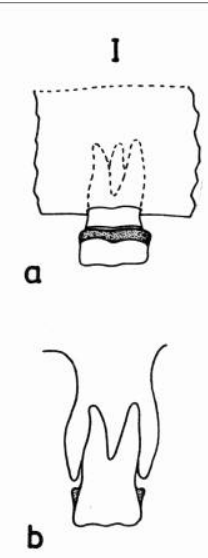
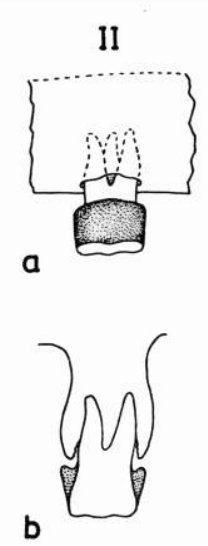
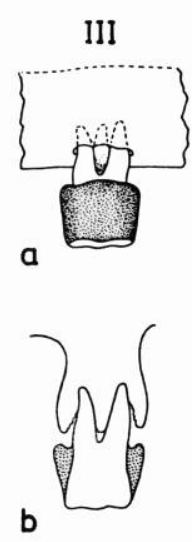
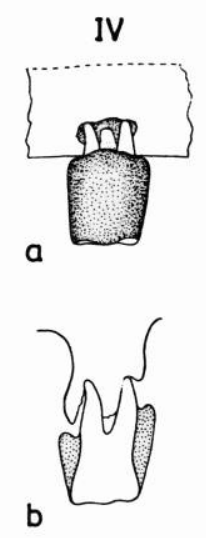
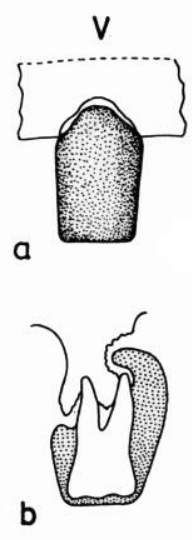

(a: Entwicklung von Zahnstein an einem Oberkiefermolaren, b: wie a, im Frontalschnitt)

Grad 0: kein Zahnstein, Grad I: leicht, Grad II: mäßig stark, Grad III: mittel stark, Grad IV: stark, Grad V: sehr stark 
Tafel 1

Abb. 1 a: Grab 281; 9,5 bis 12 Jahre. Geschlecht wahrscheinlich männlich. Ansicht von innen auf temporale Anteile des Schädels rechts. Über die Norm verstärkte Impressiones digitatae $(\rightarrow)$ in der Lamina interna als Zeichen einer Hirndrucksymptomatik.

a: Orbiatadach rechts, b: Pars petrosa ossis temporalis rechts, c: Impression des Sinus sigmoideus rechts, *: Meatus acusticus internus rechts.

Abb. 1 b: Grab 318; 7,5 bis 10 Jahre. Geschlecht wahrscheinlich männlich. Os occipitale, Dorsalansicht. Der Knochen ist nach oben hin sehr spitz ausgezogen.

a: kleiner Nahtknochen.

Abb. 1 c: Grab 318; 7,5 bis 10 Jahre. Geschlecht wahrscheinlich männlich. Os occipitale und angrenzende Ossa parietalia, Dorsalansicht. Deviation der Lambdanaht. Das Os occipitale läuft nach oben hin sehr spitz zu. Zwei Nahtknochen zwischen Occipitale und Parietale rechts. a: kleiner Nahtknochen, b: Sutura lambdoidea, c: Sutura sagittalis.

Abb. 1 d: Grab 318; 7,5 bis 10 Jahre. Geschlecht wahrscheinlich männlich. Os occipitale und Os parietale rechts, Ansicht von rechts dorsolateral auf die Lambdanaht. Nahtknochen zwischen Occipitale und Parietale rechts (a).

a: kleine Nahtknochen, b: Sutura lambdoidea. 
Tafel 1
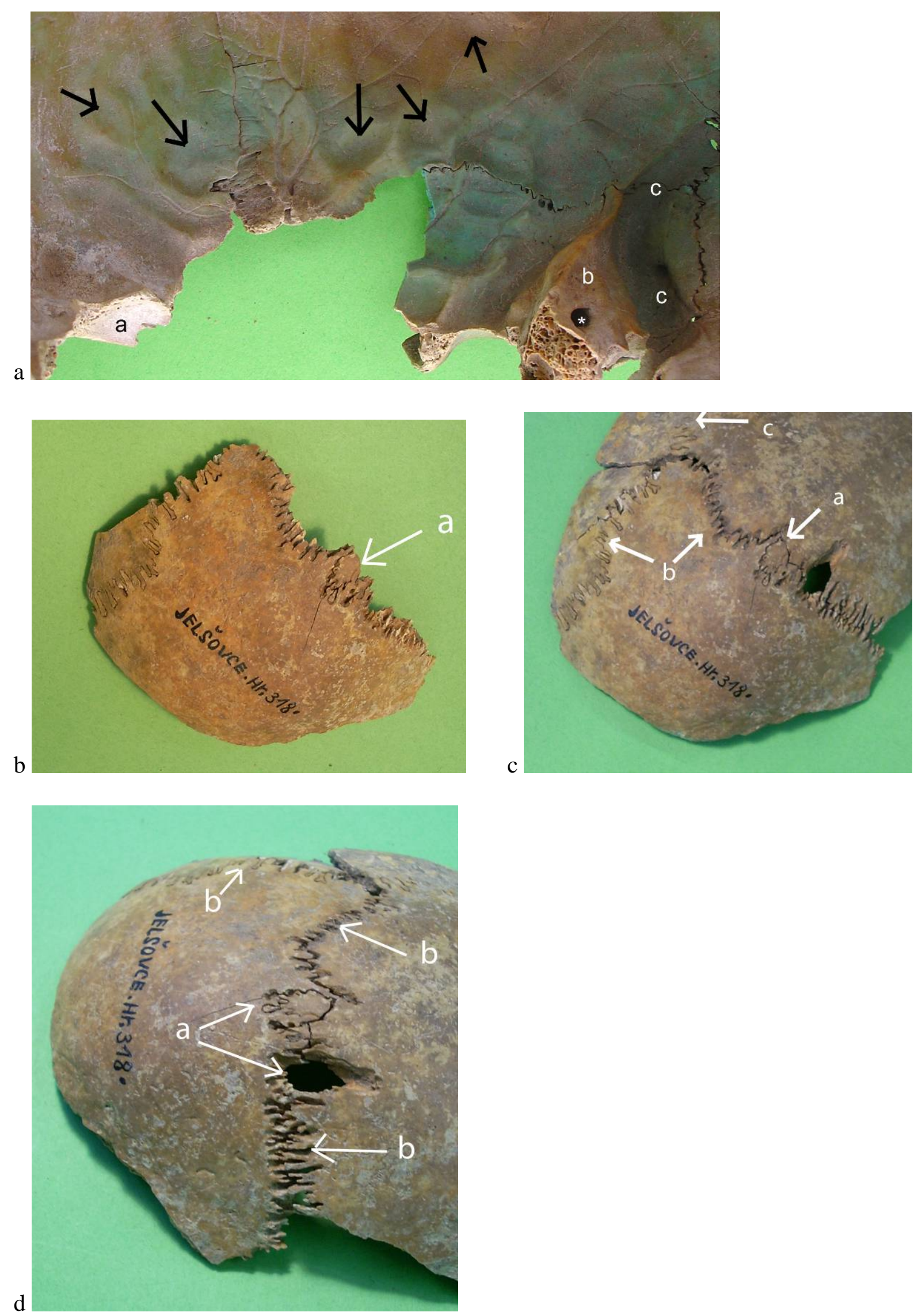
Tafel 2

Abb. 2 a: Grab 81; 1,5 bis 2,5 Jahre. Geschlecht unbestimmbar. Aufsicht auf die Lamina externa eines Fragments des linken Os parietale. Porosität der Lamina externa als frühes Zeichen einer beginnenden Anämie.

a: Lambdanaht, $\rightarrow$ b: poröses Areal.

Abb. 2 b: Grab 81; 1,5 bis 2,5 Jahre. Geschlecht unbestimmbar. Betrachtung eines Knochendünnschliffs (50 $\mu \mathrm{m})$ aus oben gezeigtem linken Os parietale im Bereich der größten Porosität im polarisierten Durchlicht unter Benutzung eines Hilfsobjekts Rot 1. Ordnung (Quarz) als Kompensator. Vergrößerung 25fach.

Es zeigt sich eine stark ausgedünnte und durchbrochene Lamina externa als frühes Zeichen einer beginnenden Anämie.

a: Lamina externa, b: versinterte Öffnung in der Lamina externa.

Abb. 2 c: Grab 81; 1,5 bis 2,5 Jahre. Geschlecht unbestimmbar. Betrachtung eines Knochendünnschliffs $(70 \mu \mathrm{m})$ aus dem linken Os parietale im polarisierten Durchlicht unter Benutzung eines Hilfsobjekts Rot 1. Ordnung (Quarz) als Kompensator. Vergrößerung 25fach..

Die Spongiosabälkchen (a) sind angedeutet vertikal zur Oberfläche hin angeordnet. Die Lamina externa ist ausgedünnt und teilweise durchbrochen.

a: vertikal ausgerichtete, hypertrophe Trabekel, b: Lamina externa, c: Lamina interna. 
Tafel 2
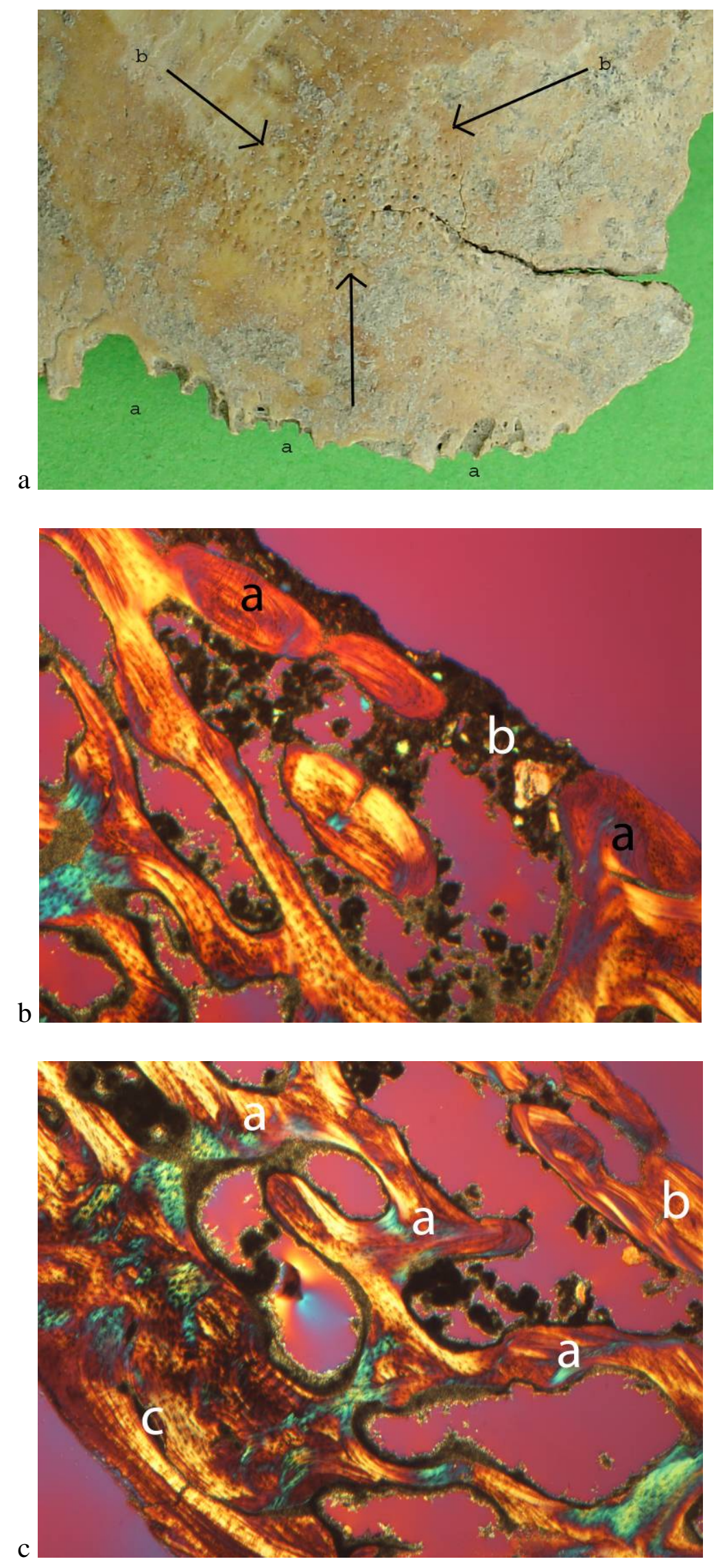
Abb. 3 a: Grab 102; 9 bis 10,5 Jahre. Geschlecht unbestimmbar. Aufsicht auf den Knochendünnschliff eines Fragments der Schädelkalotte $(50 \mu \mathrm{m})$. Vergrößerung 4,5fach. Insgesamt verbreitertes Schädeldach. Die Markräume sind verschmälert, die Spongiosabälkchen überwiegend tangential orientiert. Es besteht der Verdacht auf eine ausgeheilte, dass heißt eine klinisch nicht mehr bestehende Rachitis.

a: Lamina externa, b: Lamina interna.

Abb. 3 b und c: Grab 102; 9 bis 10,5 Jahre. Geschlecht unbestimmbar. Betrachtung eines Knochendünnschliffs $(50 \mu \mathrm{m})$ eines Fragments der Schädelkalotte im polarisierten Durchlicht (Abb. 3 b), bzw. im polarisierten Durchlicht unter Benutzung eines Hilfsobjekts Rot 1. Ordnung (Quarz) als Kompensator (Abb. 3 c). Vergrößerung 16fach.

Insgesamt verbreitertes Schädeldach mit atypisch konfigurierter Spongiosa. Die Lamina interna ist postmortal aufgesplittert.

a: Lamina externa, b: Lamina interna.

Abb. 3 d: Grab 102; 9 bis 10,5 Jahre. Geschlecht unbestimmbar. Betrachtung eines Knochendünnschliffs $(50 \mu \mathrm{m})$ eines Fragments der Schädelkalotte im polarisierten Durchlicht unter Benutzung eines Hilfsobjekts Rot 1. Ordnung (Quarz) als Kompensator. Vergrößerung 16fach.

Das Schädeldach erscheint durch einen postmortalen Sprung zusätzlich verbreitert (*). Die Spongiosa ist längsorientiert und atypisch konfiguriert. Zeichen eines aktiven Knochenumbaus bestehen nicht. Es handelt sich um eine gut abgeheilte Affektion. Es besteht der Verdacht auf eine ausgeheilte, klinisch nicht mehr bestehende Rachitis. a: Lamina externa, b: Lamina interna, *: postmortal entstandener Sprung. 
Tafel 3
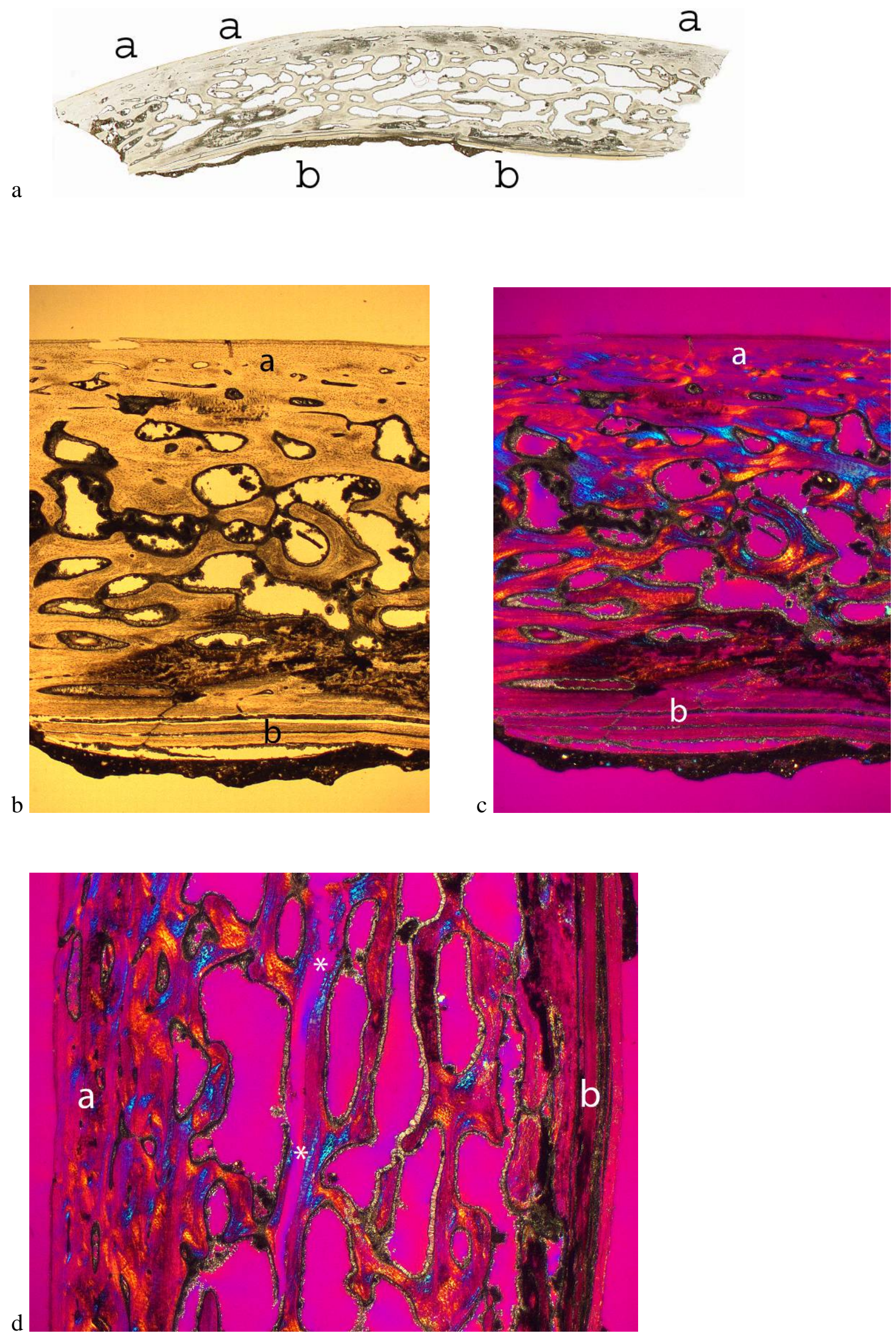
Abb. 4 a: Grab 253; 12 bis 14 Jahre. Geschlecht weiblich. Frontalansicht des Schädels. Das Kind zeigt als epigenetisches Merkmal einer Sutura metopica $(\rightarrow)$.

Abb. 4 b: Grab 253; 12 bis 14 Jahre. Geschlecht weiblich. Ansicht des Schädels von frontal oben. Zeichen eines Schädelhirntraumas. Es zeigt sich im rechten Os parietale ein Lochdefekt mit davon ausgehenden radiären Frakturlinien.

a: Frakturlinien, b: Sutura coronalis, c: Sutura sagittalis, D: traumatisch bedingter Defekt im Os parietale rechts.

Abb. 4 c: Grab 253; 12 bis 14 Jahre. Geschlecht weiblich. Ansicht des Schädels von oben. Zeichen eines Schädelhirntraumas. Es zeigt sich im rechten Os parietale ein Lochdefekt mit davon ausgehenden radiären Frakturlinien.

a: Sutura metopica, b: Sutura coronalis, c: Sutura sagittalis, D: traumatisch bedingter Defekt im Os parietale rechts.

Abb. 4 d: Grab 253; 12 bis 14 Jahre. Geschlecht weiblich. Aufsicht auf den rechten Jochbogen. Sprengung der Sutur zwischen Os zygomaticum und Maxilla rechts (Sutura zygomaticomaxillaris), Absprengung eines Teils des Os zygomaticum (c)

a: Orbita rechts, b: Sutura zygomaticofrontalis, c: Absprengung des Jochbogen im Bereich der Sutura zygomaticomaxillaris, d: Os zygomaticum.

Abb. 4 e: Grab 253; 12 bis 14 Jahre. Geschlecht weiblich. Zeichnung des Clivus. Ansicht von caudal. Beidseits perimortale Frakturlinien dorsal der Hinterhauptskondylen. 
Tafel 4
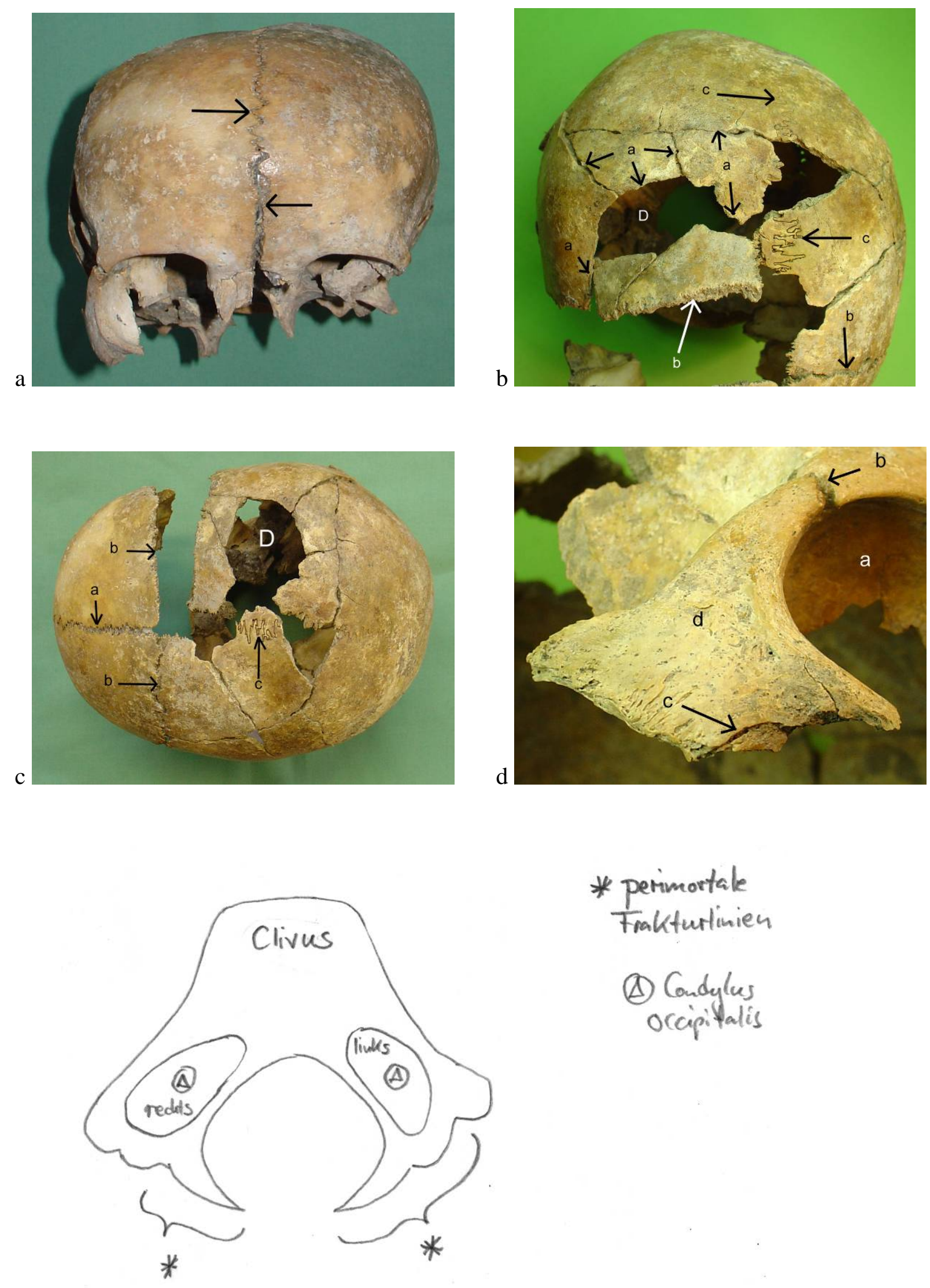

* perimortale

$$
\begin{aligned}
& \text { (4) Condylus } \\
& \text { occipitalis }
\end{aligned}
$$

e 


\section{Tafel 5}

Abb. 5 a: Grab 253; 12 bis 14 Jahre. Geschlecht weiblich. Zeichnung des Lochdefektes im Os parietale rechts. Radiär sich ausbreitende sowie konzentrisch um den Defekt liegende Frakturlinien weisen auf ein perimortales Trauma hin. Die Sprünge enden an der Sutura coronalis. Die Bruchkanten zeigen ebenfalls, dass es sich um ein perimortales Geschehen handelt.

Abb. 5 b: Grab 228 B; 6 bis 8 Jahre. Geschlecht weiblich. Röntgenaufnahme des Os frontale im seitlichen Strahlengang. Vernarbung im Os frontale $(14 \mathrm{x} 18 \mathrm{~mm})$, Strahlengang linksrechts, $52 \mathrm{kV}$, Belichtungszeit 5 Minuten.

*: Sutura coronalis, $\rightarrow$ : Mulde durch die Vernarbung.

Abb. 5 c: Grab 228 B; 6 bis 8 Jahre. Geschlecht weiblich. Ansicht von oben auf Os frontale und angrenzende Ossa parietalia. Fast runde Vernarbung im Os frontale (14 x 18mm) 11mm von Sutura coronalis entfernt.

a: Sutura coronalis, b: Sutura sagittalis, A: Os frontale, B: Os parietale links, C: Os parietale rechts, D: muldenförmige Vernarbung $(\rightarrow)$.

Abb. 5 d: Grab 19; 10 bis 12 Jahre. Geschlecht unbestimmbar. Frontalansicht des Os frontale. Foramen mit narbiger Umgebung supranasal am Os frontale.

a: Margo supraorbitalis rechts, b: Margo supraorbitalis links, c: Sutura frontonasalis, $\rightarrow$ : Foramen mit narbiger Umgebung.

Abb. 5 e: Grab 19; 10 bis 12 Jahre. Geschlecht unbestimmbar. Endocraniale Ansicht auf das Os frontale. Öffnung eines Foramens $(\rightarrow)$ in den Sulcus sinus sagittalis superior $\left(^{*}\right)$. a: Margo supraorbitalis links, b: Sutura frontonasalis. 
Tafel 5
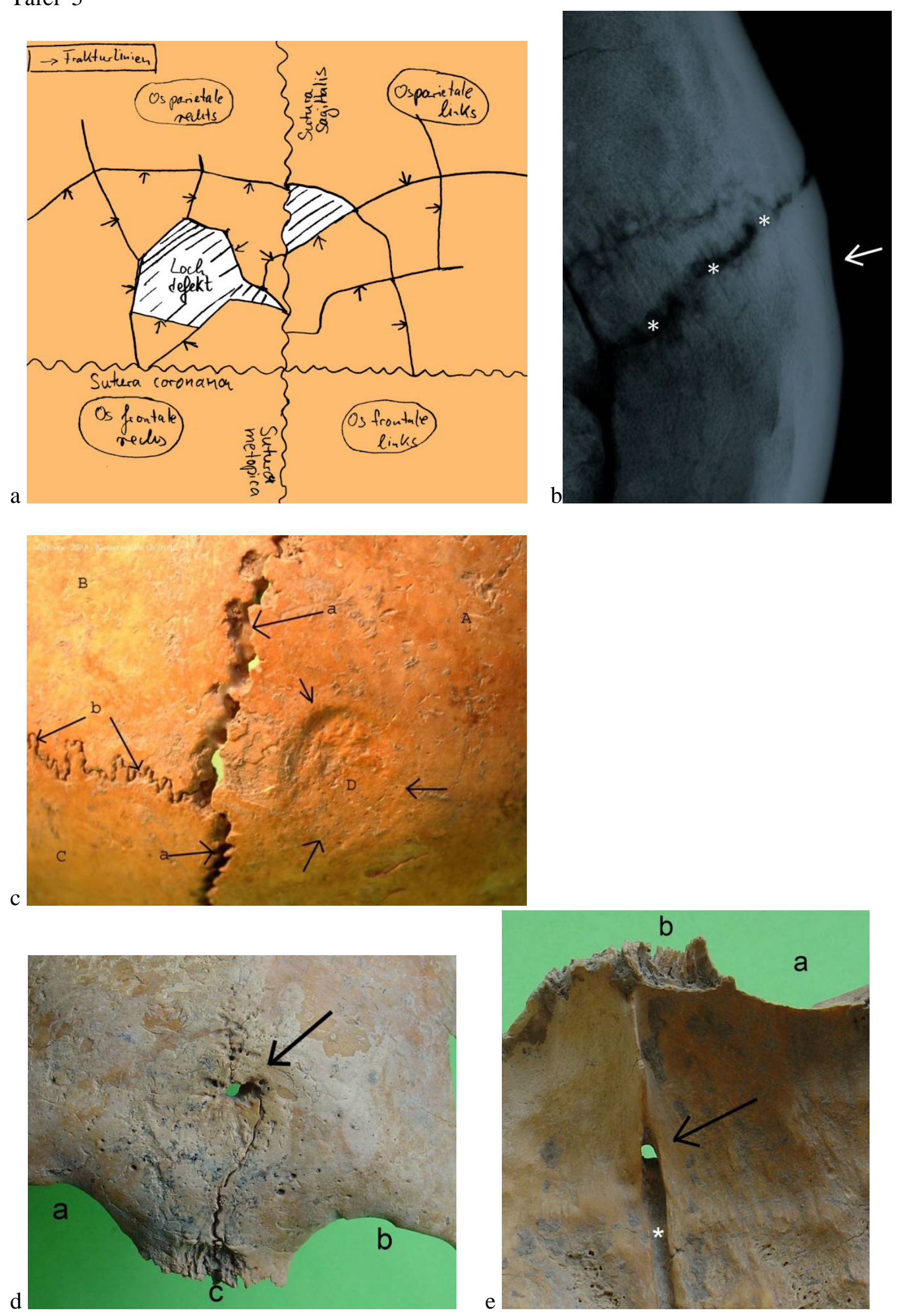
Abb. 6 a: Grab 162; 0,5 bis 1 Jahr. Geschlecht unbestimmbar. Aufsicht auf die Lamina interna des rechten Os parietale. Neben postmortaler Zerstörung sind Gefäßimpressionen und zungenförmige Platten als Zeichen eines epiduralen Hämatoms zu erkennen $(\rightarrow)$.

Abb. 6 b: Grab 162; 0,5 bis 1 Jahr. Geschlecht unbestimmbar. Lupenmikroskopische Detailaufnahme der Lamina interna des Os parietale rechts. Neben postmortaler Zerstörung sind hämorrhagisch bedingte Gefäßimpressionen und zungenförmige Platten als Zeichen eines epiduralen Hämatoms zu erkennen.

a: zungenförmige Platten, b: Gefäßimpressionen.

Abb. 6 c: Grab 162; 0,5 bis 1 Jahr. Geschlecht unbestimmbar. Vergrößerter Ausschnitt aus Abb. 6 b. Hämorrhagisch bedingte Veränderungen als Zeichen eines epiduralen Hämatoms. a: zungenförmige Platten, b: Gefäßimpressionen.

Abb. 6 d und e: Grab 162; 0,5 bis 1 Jahr. Geschlecht unbestimmbar. Betrachtung eines Knochendünnschliffs $(50 \mu \mathrm{m})$ aus dem rechten Os parietale im einfachen Durchlicht (Abb. 6 d) bzw. im polarisierten Durchlicht unter Benutzung eines Hilfsobjekts Rot 1. Ordnung (Quarz) als Kompensator (Abb. 6e). Vergrößerung 16fach.

In den Impressiones digitatae zeigen sich der ehemaligen Oberfläche aufgelagert Knochenneubildungen $(\rightarrow)$.

a: Lamina externa, b: Lamina interna, $\rightarrow$ : Impressio digitata mit hämorrhagisch bedingter Auflagerung als Zeichen eines epiduralen Hämatoms. 
Tafel 6
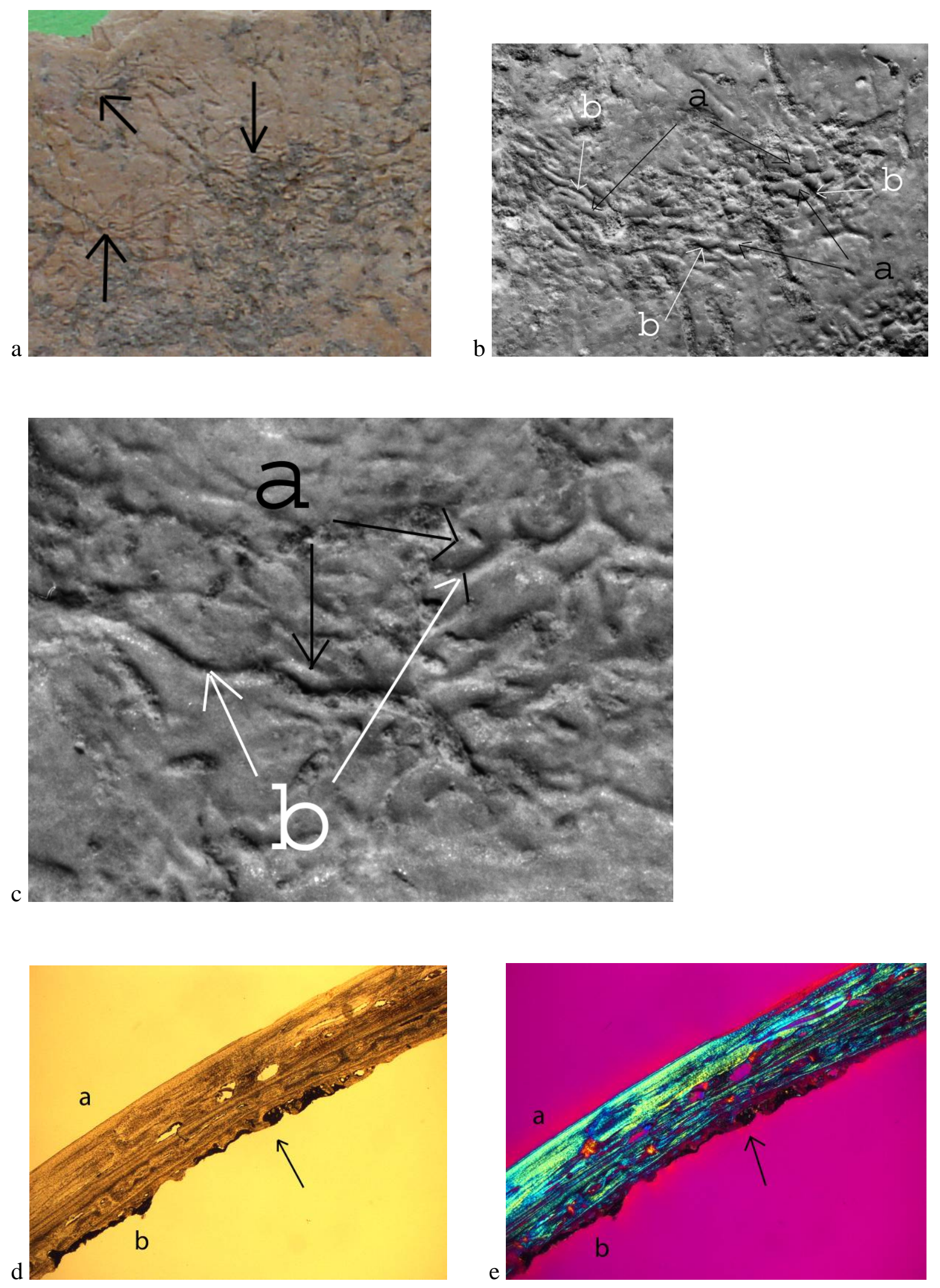


\section{Tafel 7}

Abb. 7 a: Grab 162; 0,5 bis 1 Jahr. Geschlecht unbestimmbar. Betrachtung eines Knochendünnschliffs $(50 \mu \mathrm{m})$ aus dem rechten Os parietale im polarisierten Durchlicht unter Benutzung eines Hilfsobjekts Rot 1. Ordnung (Quarz) als Kompensator. Detail aus Abb. 6 e. Vergrößerung 25fach.

Die teilweise versinterte Knochenneubildung (schwarzer $\rightarrow$ ) in der Impressio digitata ist der ehemaligen Oberfläche (weißer $\rightarrow$ ) aufgelagert. Es handelt sich um hämorrhagisch bedingte Veränderungen, die als Folge eines epiduralen Hämatoms aufgetreten sind.

a: Lamina externa, b: Lamina interna, weißer $\rightarrow$ : ehemalige Lamina interna, schwarzer $\rightarrow$ : Auflagerung.

Abb. 7 b: Grab 162; 0,5 bis 1 Jahr. Geschlecht unbestimmbar. Betrachtung eines Knochendünnschliffs $(50 \mu \mathrm{m})$ aus dem rechten Os parietale im polarisierten Durchlicht unter Benutzung eines Hilfsobjekts Rot 1. Ordnung (Quarz) als Kompensator. Detail aus Abb. 7 a. Vergrößerung 100fach.

Die alte Oberfläche $(\rightarrow)$ ist gut zu erkennen. Die Knochenneubildung weist Versinterungen auf.

$\rightarrow$ : ehemalige Lamina interna, + : Auflagerung.

Abb. 7 c: Grab 140 C; 2 bis 3 Jahre. Geschlecht unbestimmbar. Ansicht der Lamina interna des Os occipitale. Links lateral des Sulcus sinus sagittalis superioris (c) ist eine Auflagerung zu erkennen (b).

a: Lambdanaht, b: Auflagerung, c: Sulcus sinus sagittalis superioris, d: Sulcus sinus transversus links.

Abb. 7 d: Grab 140 C; 2 bis 3 Jahre. Geschlecht unbestimmbar. Lupenmikroskopische Vergrößerung der perisinusoidalen Auflagerung $(\rightarrow)$ links lateral des Sulcus sinus sagittalis superioris.

a: Sulcus sinus transversus links, b: Sulcus sinus sagittalis superioris, $\rightarrow$ : Auflagerung.

Abb. 7 e: Grab 140 C; 2 bis 3 Jahre. Geschlecht unbestimmbar. Rasterelektronenmikroskopische Aufnahme des Os occipitale im Bereich der Auflagerung links lateral des Sulcus sinus sagittalis superioris. Es zeigte sich eine intravital entstandene Gefäßimpression $(\rightarrow)$.

Vergrößerung 22fach. 
Tafel 7
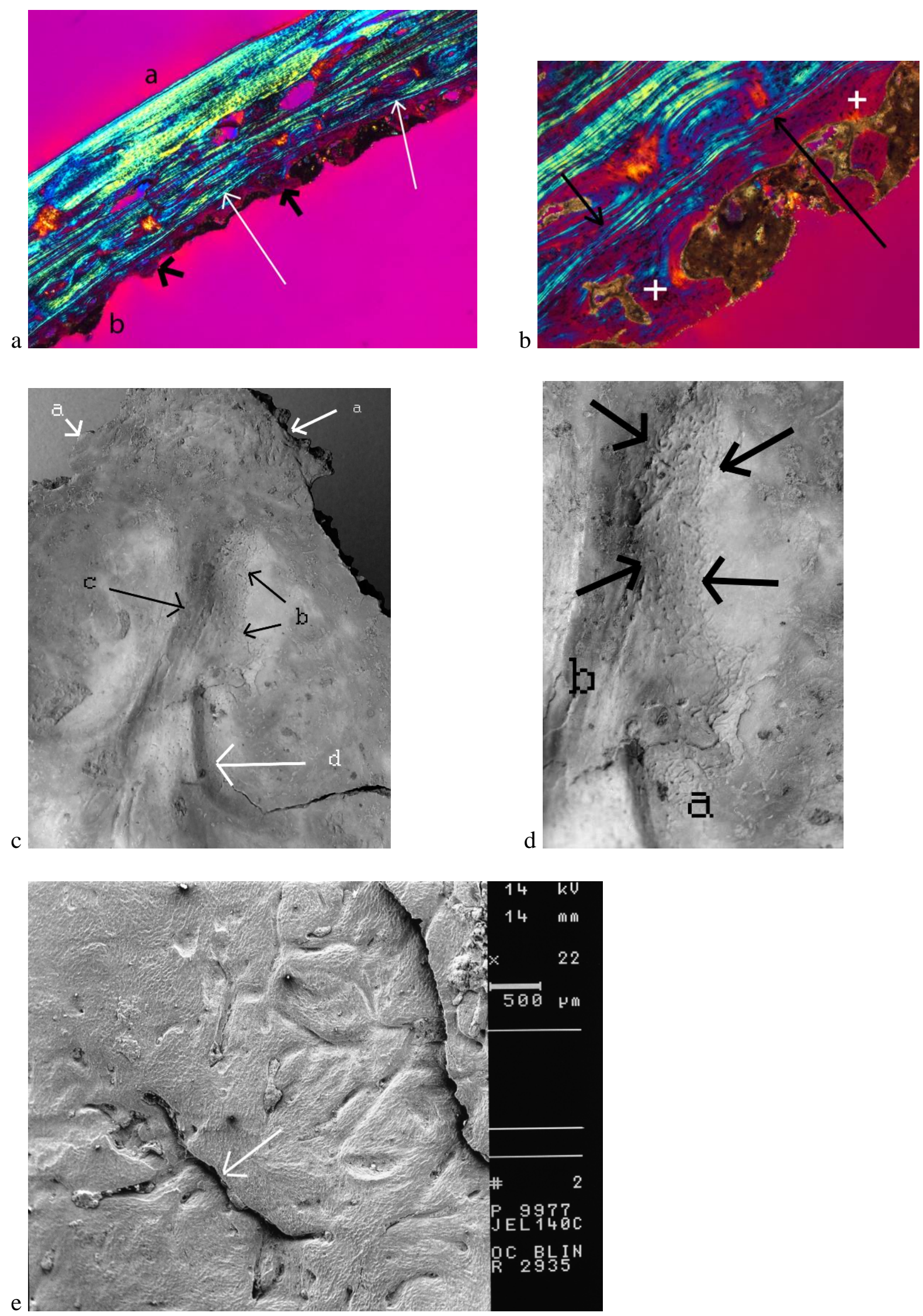
Tafel 8

Abb. 8 a: Grab 140 C; 2 bis 3 Jahre. Geschlecht unbestimmbar. Betrachtung eines Knochendünnschliffs $(50 \mu \mathrm{m})$ aus dem Os occipitale im polarisierten Durchlicht unter Benutzung eines Hilfsobjekts Rot 1. Ordnung (Quarz) als Kompensator. Vergrößerung 25fach.

Hämorrhagisch bedingte Auflagerung $(\rightarrow)$ auf der ehemaligen Oberfläche $(+)$, a: Lamina externa, b: Lamina interna, + : alte Oberfläche, $\rightarrow$ : Auflagerung.

Abb. 8 b: Grab 140 C; 2 bis 3 Jahre. Geschlecht unbestimmbar. Betrachtung eines Knochendünnschliffs $(50 \mu \mathrm{m})$ aus dem Os occipitale im polarisierten Durchlicht unter Benutzung eines Hilfsobjekts Rot 1. Ordnung (Quarz) als Kompensator. Vergrößerung 25fach.

Hämorrhagisch bedingte Auflagerung $(\rightarrow)$ auf der ehemaligen Oberfläche $(+)$

a: Lamina interna, postmortaler Abbruch der Auflagerung, b: Lamina externa, +: alte Oberfläche, $\rightarrow$ : Auflagerung.

Abb. 8 c und d: Grab 140 C; 2 bis 3 Jahre. Geschlecht unbestimmbar. Betrachtung eines Knochendünnschliffs $(50 \mu \mathrm{m})$ aus dem Os occipitale im polarisierten Durchlicht (Abb. 8 c) bzw. im polarisierten Durchlicht unter Benutzung eines Hilfsobjekts Rot 1. Ordnung (Quarz) als Kompensator (Abb. 8 d). Detail aus Abb. 8a. Vergrößerung 100fach.

Detail der Lamina interna. Die Auflagerung auf der ehemaligen Oberfläche $(\rightarrow)$ ist deutlich erkennbar. Es handelt sich um die Reste eines epiduralen Hämatoms.

Abb. 8 e: Grab 140 C; 2 bis 3 Jahre. Geschlecht unbestimmbar. Betrachtung eines Knochendünnschliffs $(70 \mu \mathrm{m})$ aus dem Os occipitale im polarisierten Durchlicht unter Benutzung eines Hilfsobjekts Rot 1. Ordnung (Quarz) als Kompensator. Vergrößerung 100fach.

Detail der Lamina interna. Im Bereich der Auflagerung ist eine intravital entstandene Gefäßimpression $(\rightarrow)$ zu erkennen.

+: ehemalige Oberfläche, $\rightarrow$ : Gefäßimpression. 
Tafel 8
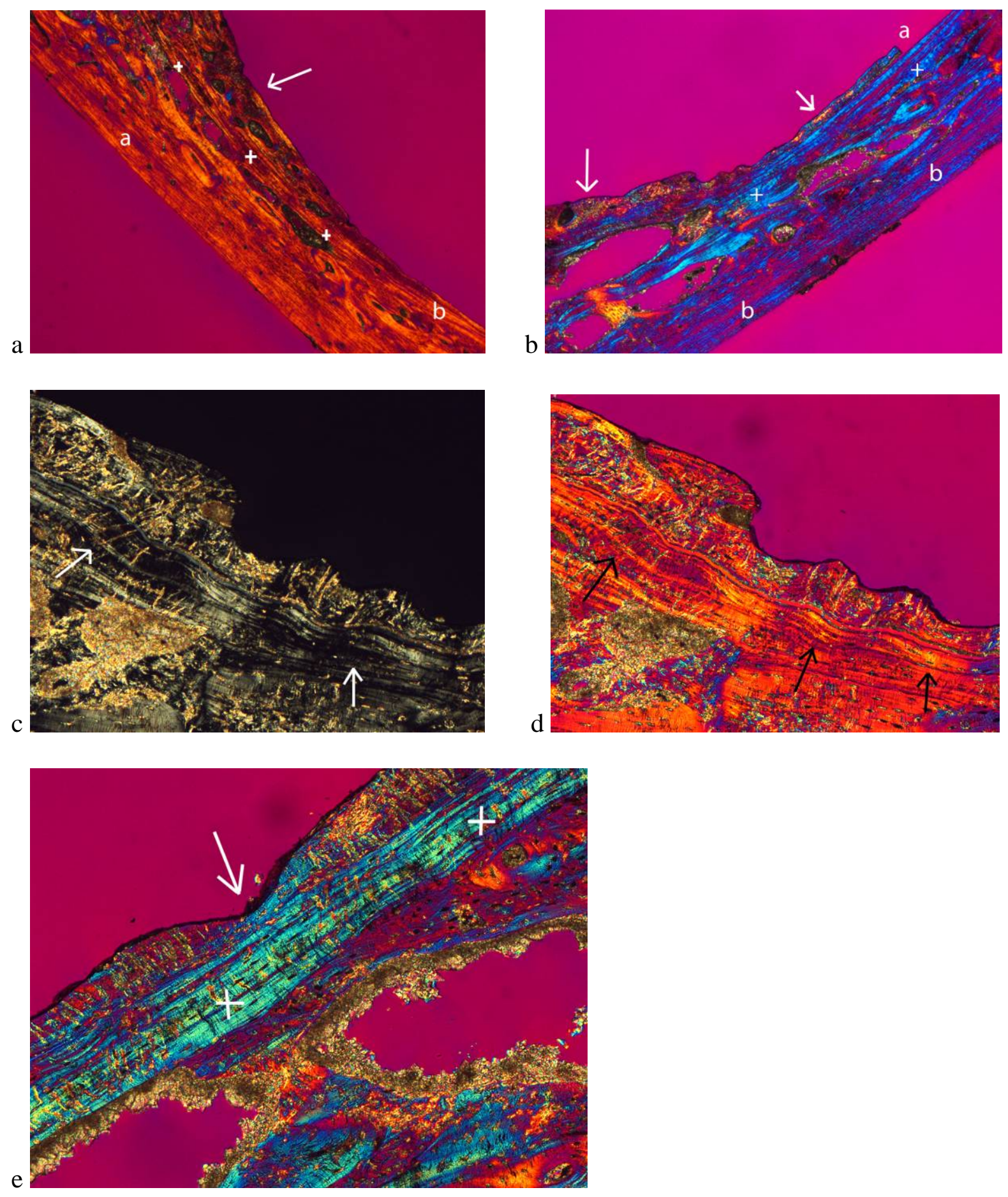
Abb. 9 a: Grab 212; 9,5 bis 12 Jahre. Geschlecht unbestimmbar. Fragment des Os occipitale, Fossa cranii posterior rechts. Caudal des Sulcus sinus transversus rechts sind kleine in Gruppen stehende Grübchen zu finden (c). Es besteht der Verdacht auf eine tuberkulöse Meningitis.

a: Lambdanaht, b: Sulcus sinus transversus rechts, c: Grübchen.

Abb. 9 b: Grab 212; 9,5 bis 12 Jahre. Geschlecht unbestimmbar. Rasterelektronenmikroskopische Aufnahme einer Grübchengruppe in der Fossa cranii posterior caudal des Sulcus sinus transversus rechts. Detail aus Abb. 9 a. Vergrößerung 10fach.

Abb. 9 c: Grab 212; 9,5 bis 12 Jahre. Geschlecht unbestimmbar. Rasterelektronenmikroskopische Aufnahme einer Grübchengruppe in der Fossa cranii posterior rechts. Detail aus Abb. 9 b. Vergrößerung 30fach.

Abb. 9 d: Grab 212, 9,5 bis 12 Jahre. Geschlecht unbestimmbar. Rasterelektronenmikroskopische Aufnahme eines Grübchens in der Fossa cranii posterior rechts. Detail aus Abb. 9 c. Vergrößerung 100fach.

Abb. 9 e und f: Grab 212; 9,5 bis 12 Jahre. Geschlecht unbestimmbar. Betrachtung eines Knochendünnschliffs $(70 \mu \mathrm{m})$ aus dem Os occipitale im Bereich der Grübchen im polarisierten Durchlicht (Abb. 9 e) bzw. im polarisierten Durchlicht unter Benutzung eines Hilfsobjekts Rot 1. Ordnung (Quarz) als Kompensator (Abb. 9 f). Vergrößerung 25fach. In der Lamina interna sind drei intravital entstandene Grübchen zu erkennen. 
Tafel 9
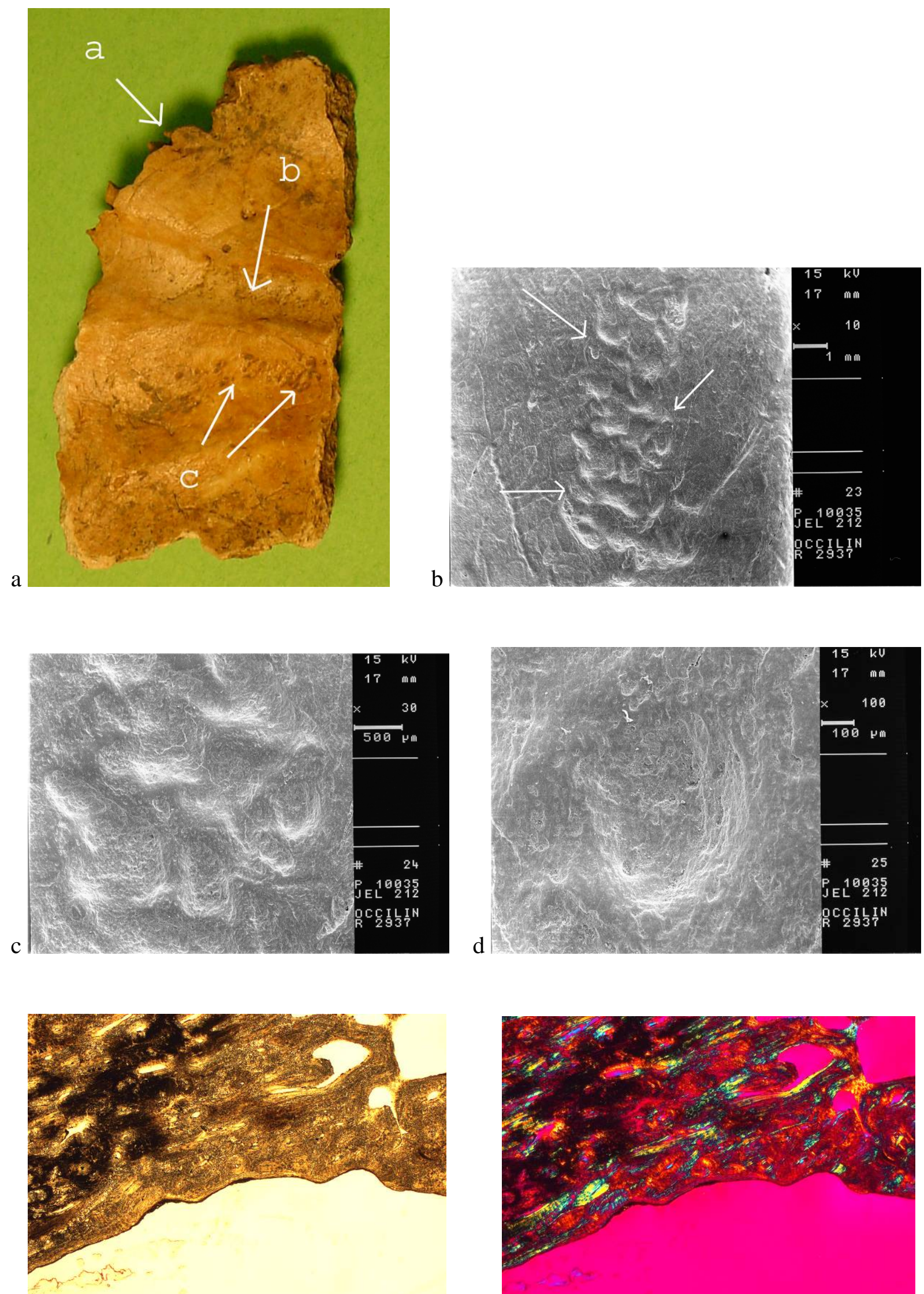

e

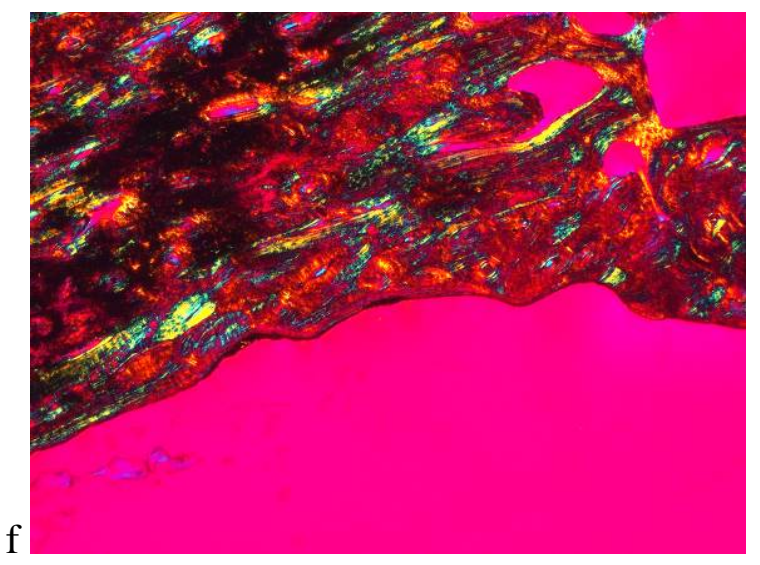


Abb. 10 a: Grab 212; 9,5 bis 12 Jahre. Geschlecht unbestimmbar. Betrachtung eines Knochendünnschliffs $(70 \mu \mathrm{m})$ aus dem Os occipitale im polarisierten Durchlicht unter Benutzung eines Hilfsobjekts Rot 1. Ordnung (Quarz) als Kompensator. Vergrößerung 25fach.

Das Grübchen $(\rightarrow)$ in der Lamina interna ist, wie der Verlauf der Kollagenfaserbündel zeigt, intravital entstanden. Diese brechen im Bereich des Grübchens nicht ab, sondern laufen außen um dieses herum. Es besteht der Verdacht auf eine tuberkulöse Meningitis.

Abb. 10 b: Grab 212; 9,5 bis 12 Jahre. Geschlecht unbestimmbar. Betrachtung eines Knochendünnschliffs $(70 \mu \mathrm{m})$ aus dem Os occipitale im polarisierten Durchlicht unter Benutzung eines Hilfsobjekts Rot 1. Ordnung (Quarz) als Kompensator. Detail aus Abb. 10 a. Vergrößerung 200fach.

Intravital entstandenes Grübchen in der Lamina interna.

Abb. 10 c: Grab 212; 9,5 bis 12 Jahre. Geschlecht unbestimmbar. Betrachtung eines Knochendünnschliffs $(50 \mu \mathrm{m})$ aus dem Os occipitale im polarisierten Durchlicht unter Benutzung eines Hilfsobjekts Rot 1. Ordnung (Quarz) als Kompensator. Vergrößerung 100fach.

Intravital entstandenes Grübchen in der Lamina interna mit postmortaler Versinterung.

Abb. 10 d: Grab 566; 5,5 bis 7 Jahre. Geschlecht unbestimmbar. Pars petrosa ossis temporalis links. Ansicht von caudal.

Am Boden des Canalis caroticus links ist eine netzartig poröse Auflagerung $(\rightarrow)$ zu finden. a: Meatus acusticus externus.

Abb. 10 e: Grab 566; 5,5 bis 7 Jahre. Geschlecht unbestimmbar. Canalis caroticus links. Endoskopische Aufnahme. Es zeigt sich die netzartig poröse Auflagerung. 
Tafel 10
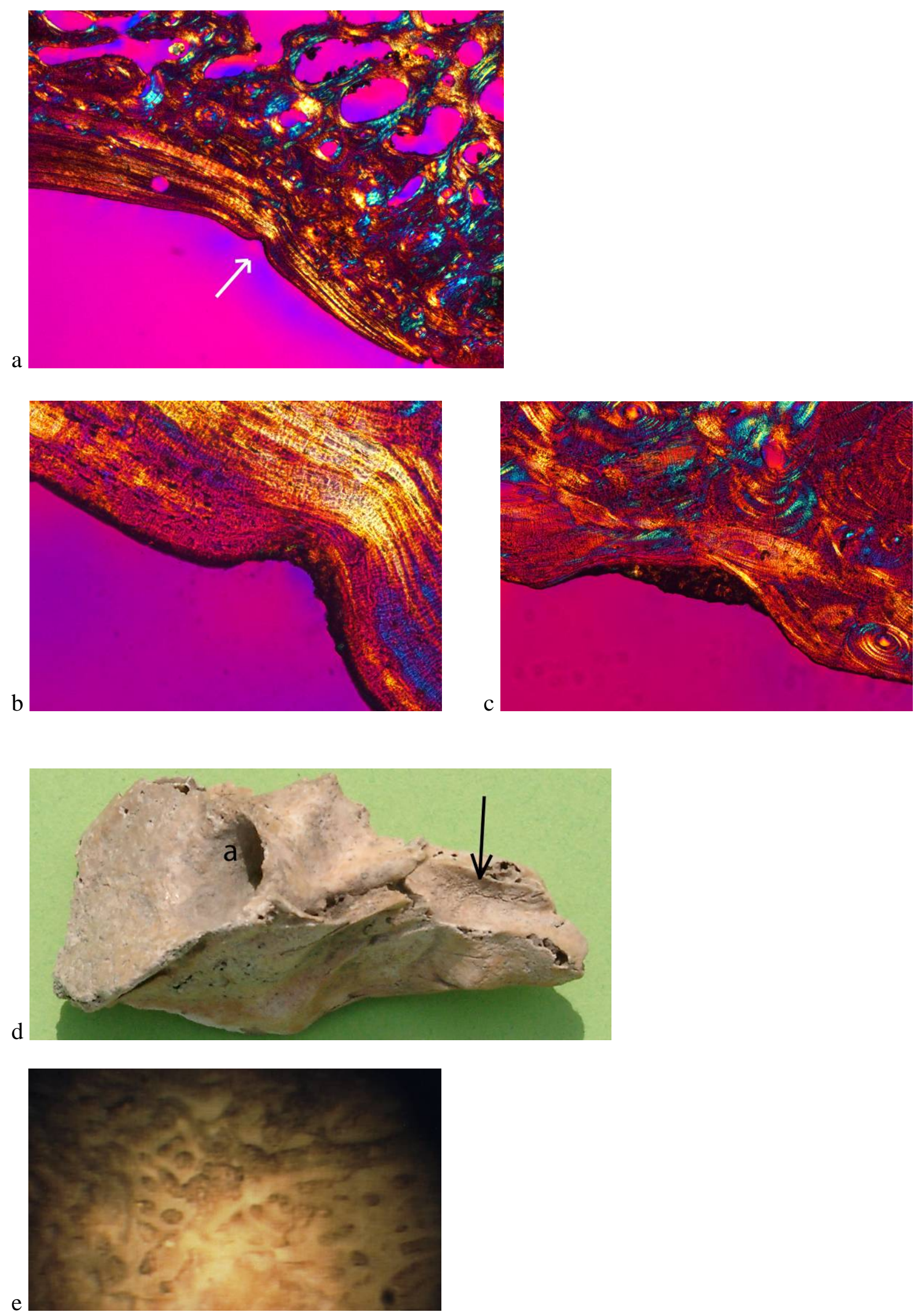
Abb. 11 a: Grab 275; 4,5 bis 6 Jahre. Geschlecht unbestimmbar. Os temporale rechts. Ansicht von lateral. Im Bereich des Processus mastoideus ist es zu einer Hemmung der Pneumatisation gekommen. Die Spitze des Processus mastoideus ist nicht pneumatisiert (a). Unmittelbar oberhalb befindet sich eine große pneumatische Zellen $(\rightarrow)$. Altersentsprechend müsste die Pneumatisation bereits bis in die Spitze des Processus mastoideus vorgedrungen sein.

a: Processus mastoideus, b: Ursprung des Arcus zygomaticus, *: Meatus acusticus externus.

Abb. 11 b: Grab 318; 7,5 bis 10 Jahre. Geschlecht wahrscheinlich männlich. Os temporale links. Ansicht von lateral. Cranial des Processus mastoideus (b) und dorsocranial des Meatus acusticus externus $(*)$ befindet sich ein kleines Foramen $(\rightarrow)$ in der Knochenoberfläche. Die Umgebung ist leicht porös. Eventuell handelt es sich um einen nach außen durchgebrochenen Mittelohrprozess (Fistel).

a: Ursprung des Arcus zygomaticus, b: Processus mastoideus, *: Meatus acusticus externus, $\rightarrow$ : Foramen (Fistel).

Abb. 11 c: Grab 318; 7,5 bis 10 Jahre. Geschlecht wahrscheinlich männlich. Sicht in den linken Sinus sigmoideus. Zum Mittelohr hin gelegen befindet sich ein rundes Foramen mit gezacktem Rand $(\rightarrow)$.Aufgrund der starken postmortalen Verfremdung ist nicht eindeutig zu sagen, ob es sich um einen intravitalen Prozess handelt. Eventuell zeigt sich hier eine Perforationsöffnung aus dem Mittelohrbereich.

a: Sinus sigmoideus, $\rightarrow$ : Foramen im Sinus sigmoideus.

Abb. 11 d: Grab 318; 7,5 bis 10 Jahre. Geschlecht wahrscheinlich männlich. Endoskopische Aufnahme des linken Sinus sigmoideus in unmittelbarer Umgebung des Foramens. Die Oberfläche ist stark porös. Auch hier ist die Oberfläche stark postmortal verfremdet, so dass nicht sicher zu sagen ist, ob diese Veränderungen intravital entstanden sind (s. Abb. 11 c). 
Tafel 11
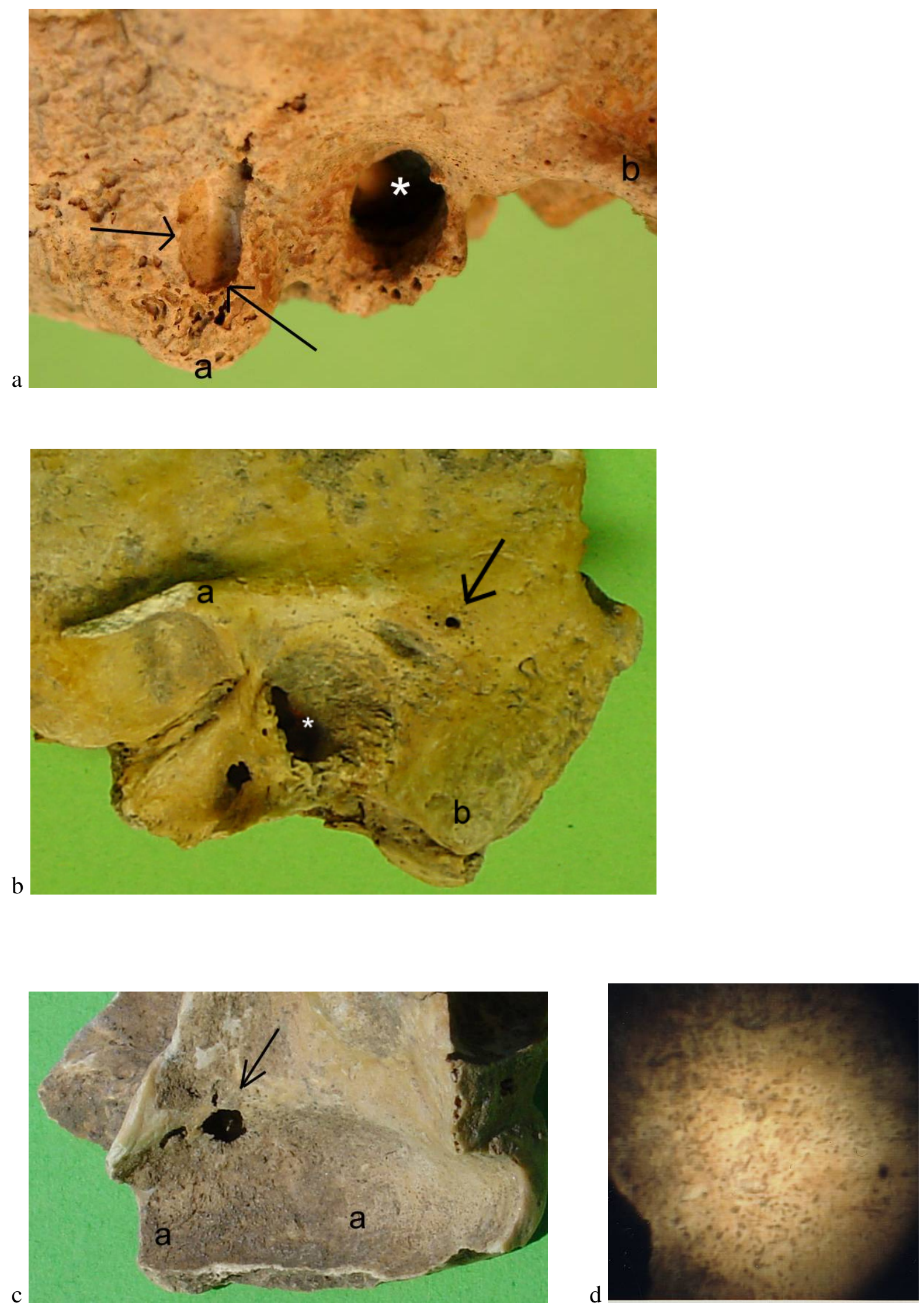
Abb. 12 a: Grab 72; 1,5 bis 2,5 Jahre. Geschlecht unbestimmbar. Aufsicht auf beide Orbitadächer. Cribra orbitalia Grad III. Beidseits sind massive poröse Auflagerungen mit Gefäßimpressionen zu erkennen.

Abb. 12 b: Grab 72; 1,5 bis 2,5 Jahre. Geschlecht unbestimmbar. Detailaufnahme des rechten Orbitadaches. Fast das gesamte Orbitadach weist eine grobporöse Oberfläche auf. Im hinteren medialen Bereich sind Gefäßimpression erkennbar.

Abb. 12 c: Grab 72; 1,5 bis 2,5 Jahre. Geschlecht unbestimmbar. Rasterelektronenmikroskopische Detailaufnahme des rechten Orbitadaches im vordersten Anteil. Die Oberfläche ist mittel- bis grobporöse, die Foramina konfluieren zum Teil. Die Neubildung scheint aus dem Orbitadach herauszuwachsen.

Vergrößerung 9fach.

Abb. 12 d: Grab 72; 1,5 bis 2,5 Jahre. Geschlecht unbestimmbar. Rasterelektronenmikroskopische Detailaufnahme des rechten Orbitadaches im hinteren Anteil. Es zeigen sich intravital entstandene, sich gabelnde Gefäßimpressionen, die von einer porösen Oberfläche umgeben sind.

Vergrößerung 25fach.

Abb. 12 e: Grab 72; 1,5 bis 2,5 Jahre. Geschlecht unbestimmbar. Rasterelektronenmikroskopische Aufnahme des rechten Orbitadaches, Detail aus Abb. 12 c. Es sind mehrere intravital entstandene Foramina zu erkennen.

Vergrößerung 80fach. 
Tafel 12
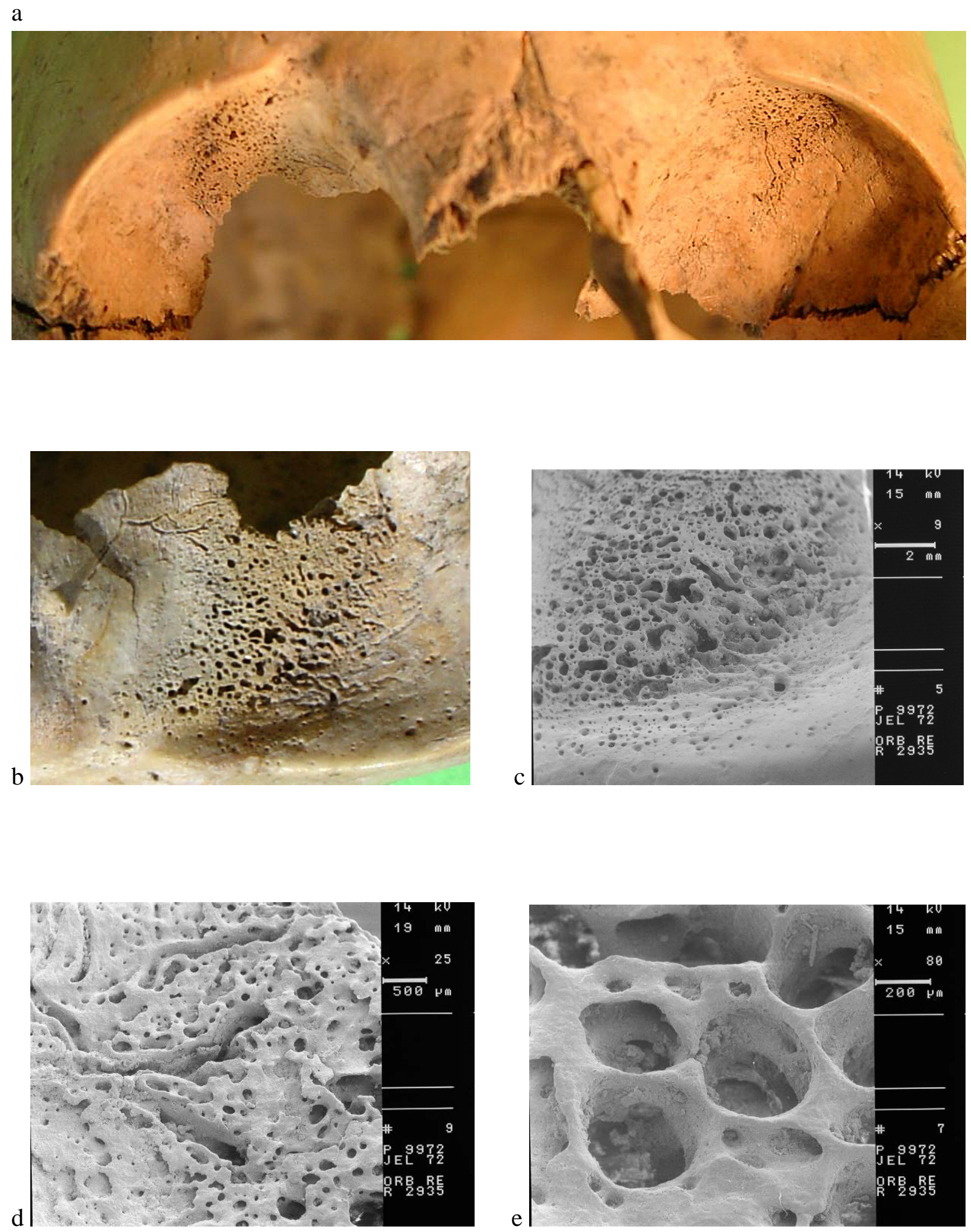
Abb. 13 a: Grab 72; 1,5 bis 2,5 Jahre. Geschlecht unbestimmbar. Betrachtung eines Knochendünnschliffs $(50 \mu \mathrm{m})$ aus dem rechten Orbitadach. Dünnschliffübersicht. Vergrößerung 3fach.

Im Bereich des Orbitadaches zeigt sich eine mehrschichtige Auflagerung mit Auflösung der ehemaligen Lamina externa.

Abb. 13 b: Grab 72; 1,5 bis 2,5 Jahre. Geschlecht unbestimmbar. Betrachtung eines Knochendünnschliffs $(50 \mu \mathrm{m})$ aus dem rechten Orbitadach im polarisierten Durchlicht unter Benutzung eines Hilfsobjekts Rot 1. Ordnung (Quarz) als Kompensator. Detailaufnahme aus dem hinteren Bereich. Vergrößerung 25fach.

Als Zeichen eines hämorrhagischen Geschehens sind mehrere parallele Schichten von Knochenneubildungen zu erkennen, die tangential zur ehemaligen Oberfläche ausgerichtet sind.

Abb. 13 c: Grab 72; 1,5 bis 2,5 Jahre. Geschlecht unbestimmbar. Betrachtung eines Knochendünnschliffs $(50 \mu \mathrm{m})$ aus dem rechten Orbitadach im polarisierten Durchlicht unter Benutzung eines Hilfsobjekts Rot 1. Ordnung (Quarz) als Kompensator. Detailaufnahme aus dem hinteren Bereich. Vergrößerung 25fach.

Aus dem Orbitadach scheinen vertikal orientierte Spongiosabälkchen heraus zu wachsen, die zum Teil in sich eine parallele horizontale Schichtung aufweisen. Hier zeigt sich die Kombination aus anämisch und hämorrhagisch bedingten Veränderungen.

Abb. 13 d: Grab 72; 1,5 bis 2,5 Jahre. Geschlecht unbestimmbar. Betrachtung eines Knochendünnschliffs $(50 \mu \mathrm{m})$ aus dem rechten Orbitadach im polarisierten Durchlicht unter Benutzung eines Hilfsobjekts Rot 1. Ordnung (Quarz) als Kompensator. Detailaufnahme aus dem mittleren Bereich. Vergrößerung 25fach.

Die Spongiosabälkchen sind vertikal orientiert und scheinen als Zeichen einer Anämie aus dem Orbitadach heraus zu wachsen. Eine durchgehende Lamina externa ist nicht vorhanden.

Abb. 13 e: Grab 72; 1,5 bis 2,5 Jahre. Geschlecht unbestimmbar. Betrachtung eines Knochendünnschliffs $(70 \mu \mathrm{m})$ aus dem rechten Orbitadach im polarisierten Durchlicht unter Benutzung eines Hilfsobjekts Rot 1. Ordnung (Quarz) als Kompensator. Detailaufnahme aus dem hinteren Bereich. Vergrößerung 25fach.

Die vertikal ausgerichteten Bälkchen sind durch mehrere horizontal orientierte Schichten aufgegliedert. Es zeigt sich die Kombination aus anämisch und hämorrhagisch bedingten Veränderungen. 
Tafel 13
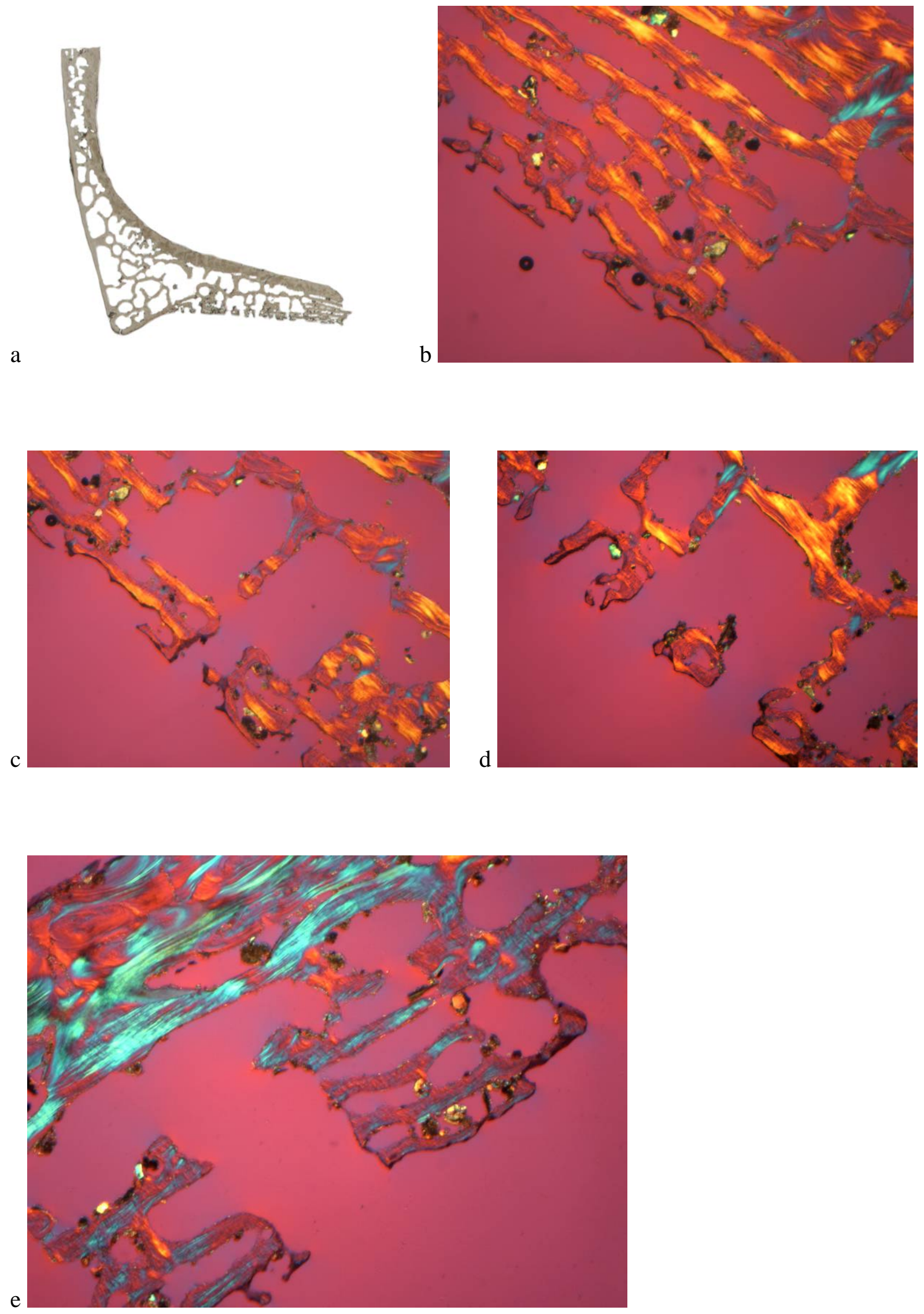
Abb. 14 a: Grab 336 B; 2 bis 4 Jahre. Geschlecht unbestimmbar. Rechtes Orbitadach. Cribra orbitalia Grad II. Die Oberfläche ist besonders im vorderen Anteil grobporös.

Abb. 14 b: Grab 336 B; 2 bis 4 Jahre. Geschlecht unbestimmbar. Linkes Orbitadach. Cribra orbitalia Grad II. Die Oberfläche ist grob- bis mittelporös.

Abb. 14 c: Grab 336 B; 2 bis 4 Jahre. Geschlecht unbestimmbar. Betrachtung eines Knochendünnschliffs $(50 \mu \mathrm{m})$ aus dem rechten Orbitadach im polarisierten Durchlicht unter Benutzung eines Hilfsobjekts Rot 1. Ordnung (Quarz) als Kompensator. Detailaufnahme aus dem hinteren Bereich. Vergrößerung 16fach.

Die Lamina externa des Orbitadaches ist stark diagenetisch verändert. Die oberflächennahe Spongiosa erscheint reduziert, der Markraum ist leicht unregelmäßig konfiguriert und vergrößert. Es besteht der Verdacht auf einen entzündlichen Prozess.

Abb. 14 d: Grab 336 B; 2 bis 4 Jahre. Geschlecht unbestimmbar. Betrachtung eines Knochendünnschliffs $(50 \mu \mathrm{m})$ aus dem rechten Orbitadach im polarisierten Durchlicht unter Benutzung eines Hilfsobjekts Rot 1. Ordnung (Quarz) als Kompensator. Detailaufnahme aus dem hinteren Bereich. Detail aus Abb. 14 c. Vergrößerung 25fach.

Stark diagenetisch veränderter Bereich der Lamina externa. Die eigentliche Lamina externa ist weitestgehend aufgelöst. 
Tafel 14
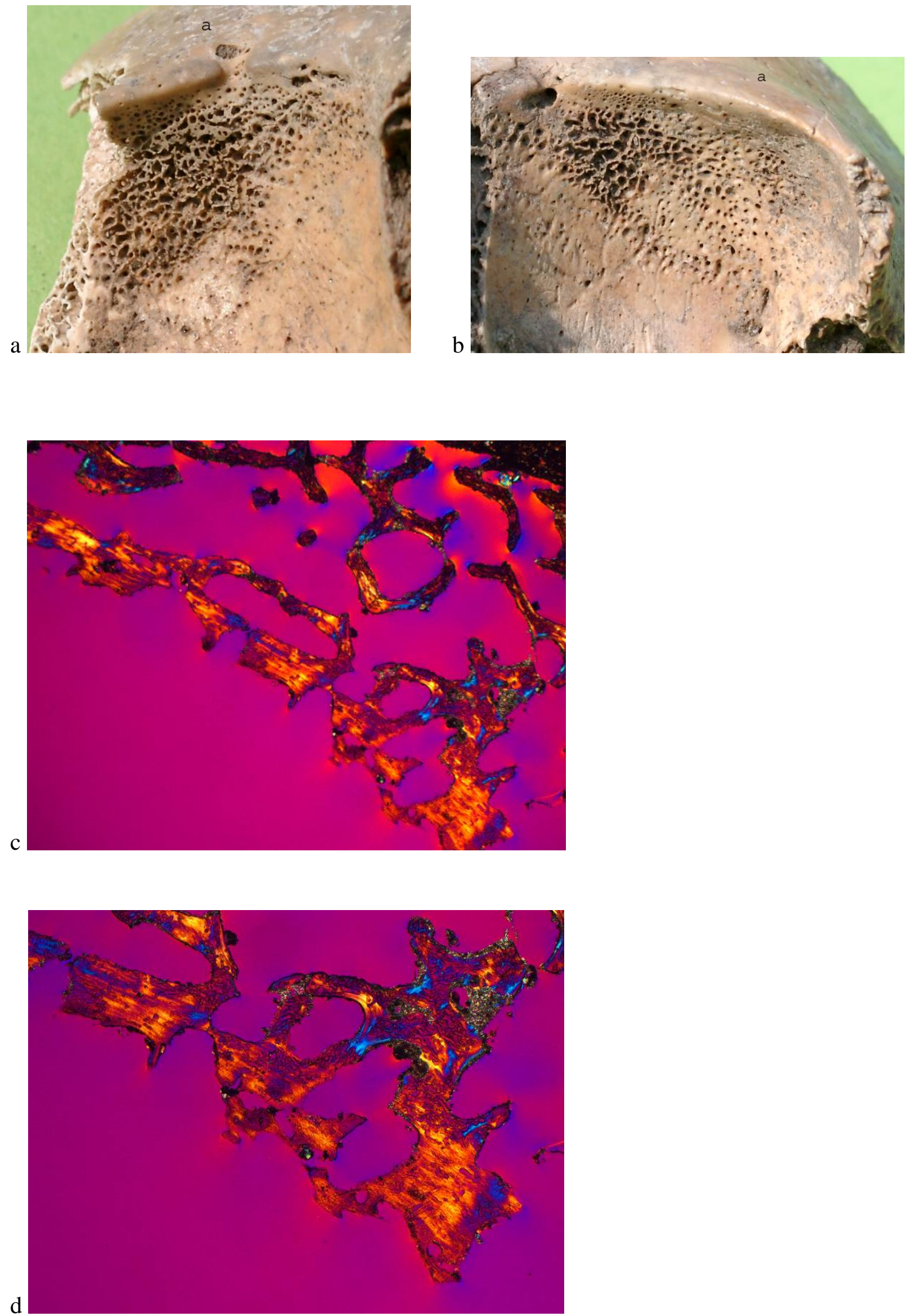
Abb. 15 a: Grab 88 A; 2,5 bis 4,5 Jahre. Geschlecht unbestimmbar. Aufsicht auf das rechte Orbitadach. Die Oberfläche ist über die Norm vermehrt porös.

Abb. 15 b: Grab 88 A; 2,5 bis 4,5 Jahre. Geschlecht unbestimmbar. Betrachtung eines Knochendünnschliffs $(50 \mu \mathrm{m})$ aus dem rechten Orbitadach im polarisierten Durchlicht unter Benutzung eines Hilfsobjekts Rot 1. Ordnung (Quarz) als Kompensator. Detailaufnahme aus dem vorderen Bereich. Vergrößerung 25fach.

Es zeigt sich eine postmortale Cribra orbitalia. Zahlreiche Trabekel sind postmortal abgebrochen $(\rightarrow)$. Die Knochenmarksräume $(*)$ sind nicht erweitert. Hinweise auf eine Anämie sind nicht zu finden.

Abb. 15 c: Grab 88 A; 2,5 bis 4,5 Jahre. Geschlecht unbestimmbar. Betrachtung eines Knochendünnschliffs $(70 \mu \mathrm{m})$ aus dem rechten Os frontale (supraorbital) im polarisierten Durchlicht unter Benutzung eines Hilfsobjekts Rot 1. Ordnung (Quarz) als Kompensator. Vergrößerung 25fach.

Die Diploë ist dystrophisch. Die Spongiosadystrophie äußert sich in sehr grazilen, schräg parallel zur Oberfläche verlaufenden Spongiosabälkchen. Die Knochenmarksräume sind verschmälert. Diese Veränderungen sind Hinweis auf einen entzündlichen Prozess, der vermutlich von Sinus frontalis derselben Seite ausging.

a: Lamina externa, frontal, b: Lamina interna, endocranial, *: schräge Ausrichtung der Markräume.

Abb. 15 d: Grab 126; 9 bis 11 Jahre. Geschlecht weiblich. Linkes Orbitadach. Es zeigt sich eine grobporöse Cribra orbitalia.

Abb. 15 e: Grab 126; 9 bis 11 Jahre. Geschlecht weiblich. Betrachtung eines Knochendünnschliffs $(50 \mu \mathrm{m})$ aus dem linken Orbitadach im polarisierten Durchlicht unter Benutzung eines Hilfsobjekts Rot 1. Ordnung (Quarz) als Kompensator. Vergrößerung 16fach.

Die Cribra ist zum größten Teil postmortal entstanden. Zahlreiche Trabekel sind postmortal abgebrochen. Im hinteren Anteil sind die Trabekel angedeutet senkrecht zur Oberfläche ausgerichtet. Darüber liegen zum Teil relativ große Spongiosaräume.

Abb. 15 f: Grab 126; 9 bis 11 Jahre. Geschlecht weiblich. Betrachtung eines Knochendünnschliffs $(50 \mu \mathrm{m})$ aus dem linken Orbitadach im polarisierten Durchlicht unter Benutzung eines Hilfsobjekts Rot 1. Ordnung (Quarz) als Kompensator. Detail aus Abb. 15 e. Vergrößerung 25fach.

Im hinteren Anteil sind die Trabekel angedeutet senkrecht zur Oberfläche ausgerichtet $(\rightarrow)$. Wahrscheinlich handelt es sich um sehr frühe Zeichen einer beginnenden Anämie. 
Tafel 15
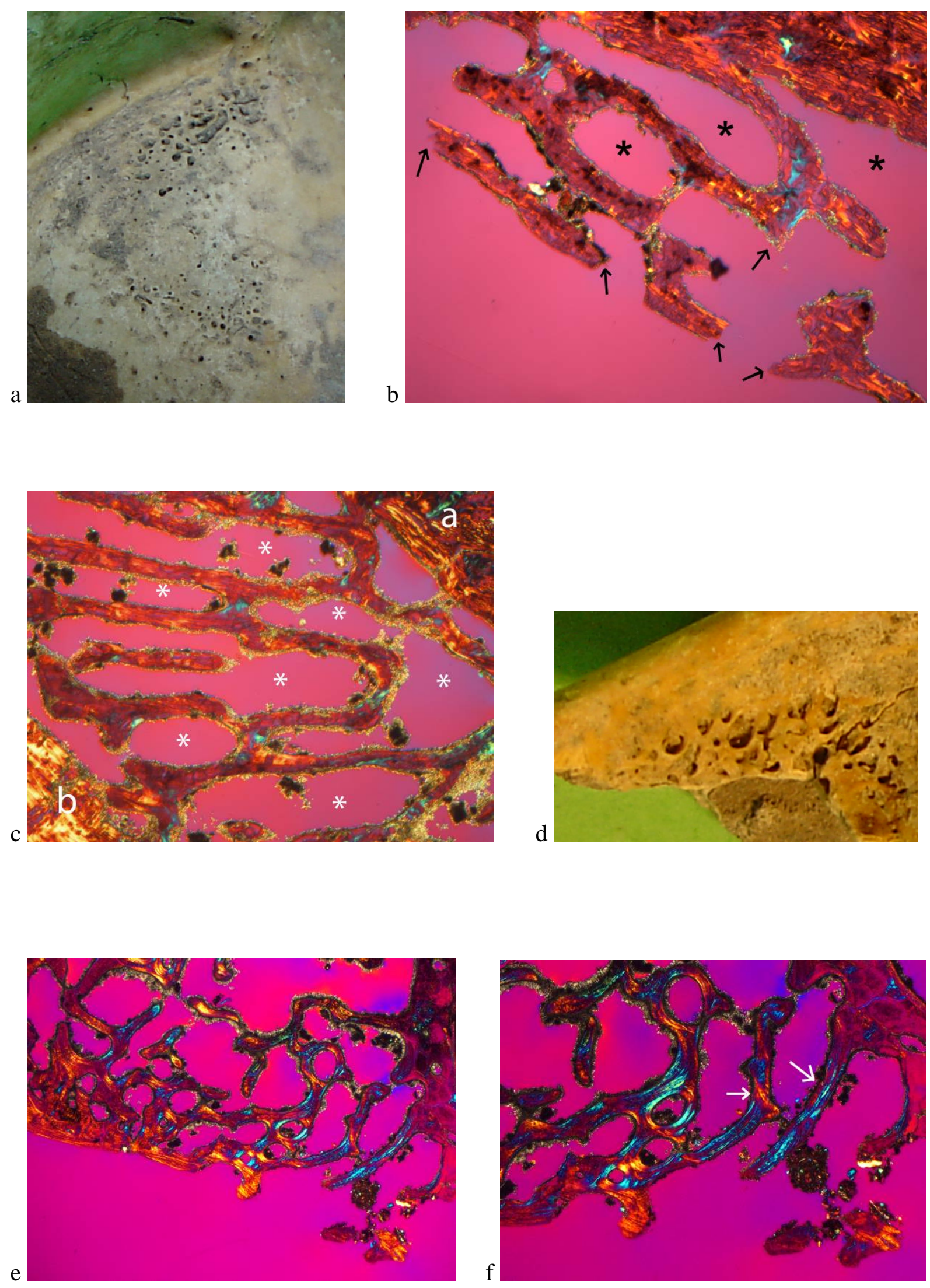
Tafel 16

Abb. 16 a: Grab 300; 2 bis 3 Jahre. Geschlecht nicht bestimmbar. Betrachtung eines Knochendünnschliffs $(50 \mu \mathrm{m})$ aus dem rechten Orbitadach im polarisierten Durchlicht unter Benutzung eines Hilfsobjekts Rot 1. Ordnung (Quarz) als Kompensator. Vergrößerung 25fach.

Als Zeichen einer Hypervaskularisation sind zahlreiche intravital entstandene Gefäßimpressionen zu erkennen.

$\rightarrow$ : Gefäßimpressionen.

Abb. 16 b: Grab 300; 2 bis 3 Jahre. Geschlecht nicht bestimmbar. Betrachtung eines Knochendünnschliffs $(50 \mu \mathrm{m})$ aus dem rechten Orbitadach im polarisierten Durchlicht unter Benutzung eines Hilfsobjekts Rot 1. Ordnung (Quarz) als Kompensator. Detail aus Abb. 16 a. Vergrößerung 100fach.

Es zeigen sich zwei intravital entstandene Gefäßimpressionen.

Abb. 16 c und d: Grab 299; 9 bis 10,5 Jahre. Geschlecht nicht bestimmbar. Betrachtung eines Knochendünnschliffs $(70 \mu \mathrm{m})$ aus dem rechten Orbitadach im einfachen Durchlicht (Abb. 16 c) bzw. im polarisierten Durchlicht unter Benutzung eines Hilfsobjekts Rot 1. Ordnung (Quarz) als Kompensator (Abb. 16 d). Vergrößerung 16fach.

Als Zeichen einer Hypervaskularisation sind zahlreiche intravital entstandene Gefäßimpressionen zu erkennen. Eventuell handelt es sich um eine entzündlich bedingte Hypervaskularisation.

$\rightarrow$ : Gefäßimpressionen.

Abb. 16 e: Grab 299; 9 bis 10,5 Jahre. Geschlecht nicht bestimmbar. Betrachtung eines Knochendünnschliffs $(70 \mu \mathrm{m})$ aus dem rechten Orbitadach im polarisierten Durchlicht unter Benutzung eines Hilfsobjekts Rot 1. Ordnung (Quarz) als Kompensator. Vergrößerung 25fach.

Knochendünnschliff des rechten Orbitadaches. Detail aus Abb. 16 d. Es sind mehrere intravital entstandene Gefäßimpressionen erkennbar.

$\rightarrow$ : Gefäßimpressionen

Abb. 16 f: Grab 299; 9 bis 10,5 Jahre. Geschlecht nicht bestimmbar. Betrachtung eines Knochendünnschliffs $(50 \mu \mathrm{m})$ aus dem rechten Orbitadach im polarisierten Durchlicht unter Benutzung eines Hilfsobjekts Rot 1. Ordnung (Quarz) als Kompensator. Vergrößerung 16fach.

Knochendünnschliff des rechten Orbitadaches. Die Diploë weist zum Teil relativ große Spongiosaräume auf $(+)$. Diese erweiterten Spongiosaräume können eventuell als Zeichen einer beginnenden Anämie gewertet werden. 
Tafel 16
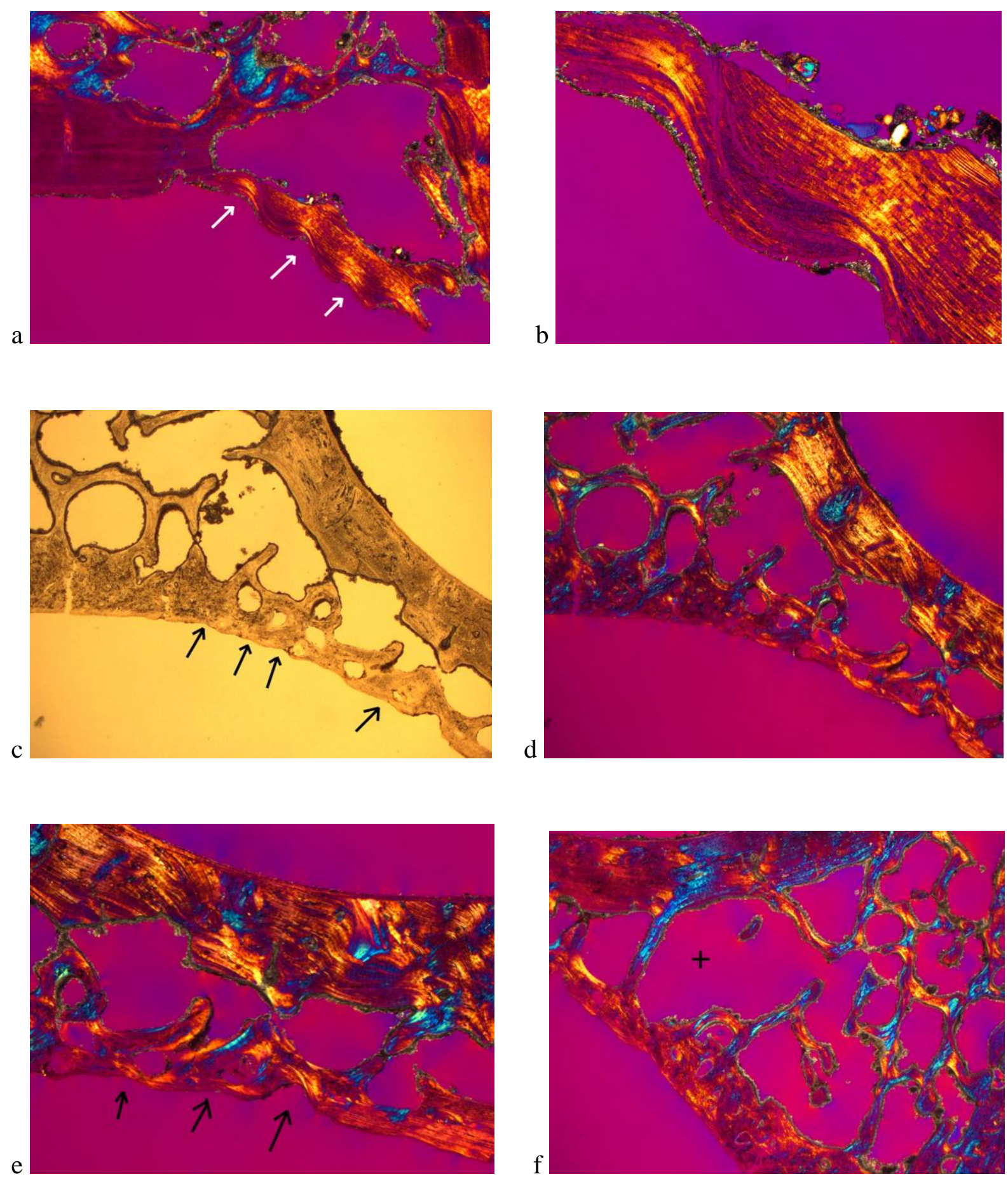
Abb. 17 a: Grab 65 A; 3 bis 4 Jahre. Geschlecht nicht bestimmbar. Blick von dorsal in den aufgebrochenen Sinus maxillaris der rechten Seite. Der Boden ist als Zeichen einer Entzündung wulstig porös verändert.

Abb. 17 b: Grab 65 A; 3 bis 4 Jahre. Geschlecht nicht bestimmbar. Endoskopische Aufnahme der rechten Sinus maxillaris. Der Boden des Recessus frontalis ist als Zeichen einer chronischen Entzündung wulstig vernarbt.

Abb. 17 c: Grab 334; 12 bis 14 Jahre. Geschlecht wahrscheinlich männlich. Blick von cranial in den aufgebrochenen Sinus maxillaris der rechten Seite. Der Boden ist als Zeichen einer Entzündung über die Norm verstärkt porös.

a: Nasenboden rechts, b: Boden des Sinus maxillaris rechts.

Abb. 17 d: Grab 334; 12 bis 14 Jahre. Geschlecht wahrscheinlich männlich. Endoskopische Aufnahme des rechten Sinus maxillaris. Der Boden ist als Zeichen einer Entzündung über die Norm verstärkt porös.

Abb. 17 e: Grab 65 A; 3 bis 4 Jahre. Geschlecht nicht bestimmbar. Blick auf den harten Gaumen. Die Oberfläche ist als Zeichen einer Stomatitis über die Norm verstärkt porös. 
Tafel 17
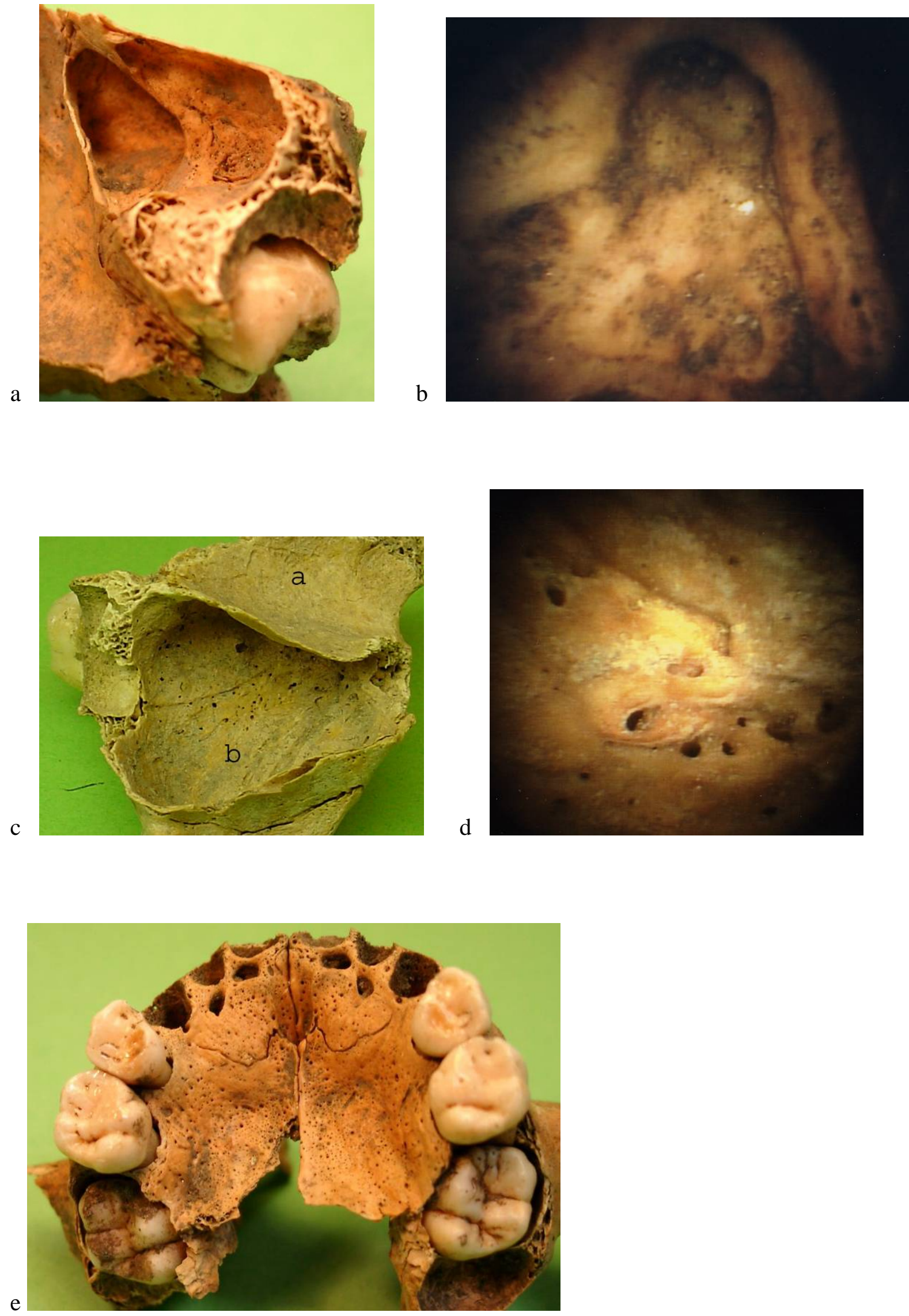
Abb. 18 a: Grab 241; 3 bis 5 Jahre. Geschlecht nicht bestimmbar. Endoskopische Aufnahme der rechten Paukenhöhle: Promontorium. Die normalerweise glatte Oberfläche ist narbig verändert. Es sind Wulstungen und grübenförmige Vertiefungen als Zeichen einer abgelaufenen Entzündung erkennbar.

Abb. 18 b: Grab 65 A; 3 bis 4 Jahre. Geschlecht nicht bestimmbar. Endoskopische Aufnahme der rechten Paukenhöhle: Promontorium. Vernarbungen in Form von grübenförmigen Vertiefungen und kleinen Einziehungen weisen auf eine chronische Mittelohrentzündung hin.

Abb. 18 c: Grab 616 B; 9 bis 11 Jahre. Geschlecht weiblich. Rechte Clavicula. Blick auf die Metapyphse des sternalen Endes. Die Oberfläche ist nicht glatt begrenzt, wie zu erwarten wäre, sondern ist als Zeichen einer Dysplasie grobporös und weist Einziehungen auf.

Abb. 18 d: Grab 616 B; 9 bis 11 Jahre. Geschlecht weiblich. Linke Clavicula. Blick auf die Metapyphse des sternalen Endes. Die Oberfläche ist nicht glatt begrenzt, wie zu erwarten wäre, sondern ist als Zeichen einer Dysplasie grobporös und weist Einziehungen auf.

Abb. 18 e: Grab 616 B; 9 bis 11 Jahre. Geschlecht weiblich. Röntgenaufnahme der rechten Clavicula. Strahlengang cranial nach caudal, 35kV, Belichtungszeit 4 Min.

Pathologische Veränderungen sind nicht zu erkennen. Es gibt keinen Hinweis auf eine ausgeheilte Fraktur.

a: acromiales Ende, b: sternales Ende, $\rightarrow$ postmortale Frakturlinie 
Tafel 18
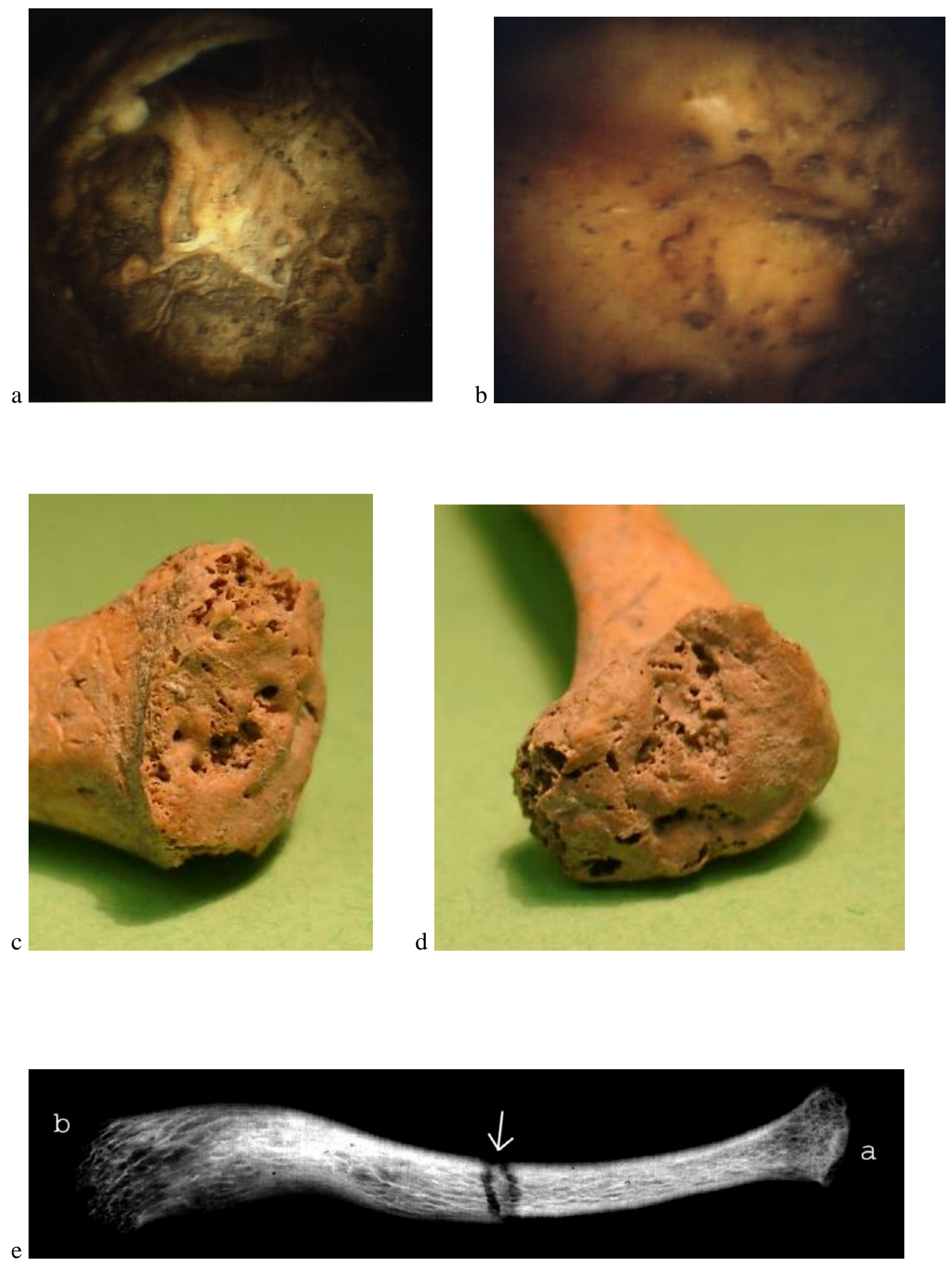
Abb. 19 a: Grab 83; 5,5 bis 7 Jahre. Geschlecht nicht bestimmbar. Mandibula links. An der Krone des Zahnes 75 sind zwei kariöse Läsionen, occlusal (a) und buccal (b), gelegen.

Abb. 19 b: Grab 616 B; 9 bis 11 Jahre. Geschlecht weiblich. Seitansicht der Zähne 64 und 65. Die kariösen Läsionen der benachbarten Zähne liegen an deren Kontaktfläche $(\rightarrow)$ (bei 64 an der Krone distal, bei 65 an der Krone mesial).

Abb. 19 c: Grab 336 B; 2 bis 4 Jahre. Geschlecht nicht bestimmbar. Buccalansicht der linken Maxilla. Als Zeichen einer Parodontose ist der Alveolarknochen vor allem über den Zähnen 61 und 62 deutlich reduziert. Die Zahnhälse sind stärker als üblich sichtbar.

Abb. 19 d: Grab 99; 6 bis 8 Jahre. Geschlecht nicht bestimmbar. Buccalansicht der rechten Maxilla. Als Zeichen einer Parodontose sind die Zahnhälse der Zähne 53 bis 55 stärker als üblich sichtbar.

Abb. 19 e: Grab 254 B; 7 bis 10 Jahre. Geschlecht nicht bestimmbar. Buccalansicht der rechten Maxilla. Als Zeichen einer Parodontose sind Zahnhälse und Wurzeln der Zähne 53 bis 55 stärker als üblich sichtbar $(\rightarrow)$. Außerdem zeigt sich eine Fehlstellung der bleibenden Zähne 11 und 12. Zahn 12 steht in leichter schräg distal von Zahn 11.

Abb. 19 f: Grab 220; 9,5 bis 12,5 Jahre. Geschlecht weiblich. Mandibula, Ansicht von rechts vorne. An den Zähnen 31, 32, 41, 42 und 43 sind transversale Schmelzhypoplasien in Gestalt von feinen Rillen zu erkennen. An der frontalen Fläche des Zahnes 31 sowie mesial an den Zähnen 31 und 41 befinden sich Reste von Zahnstein. 
Tafel 19
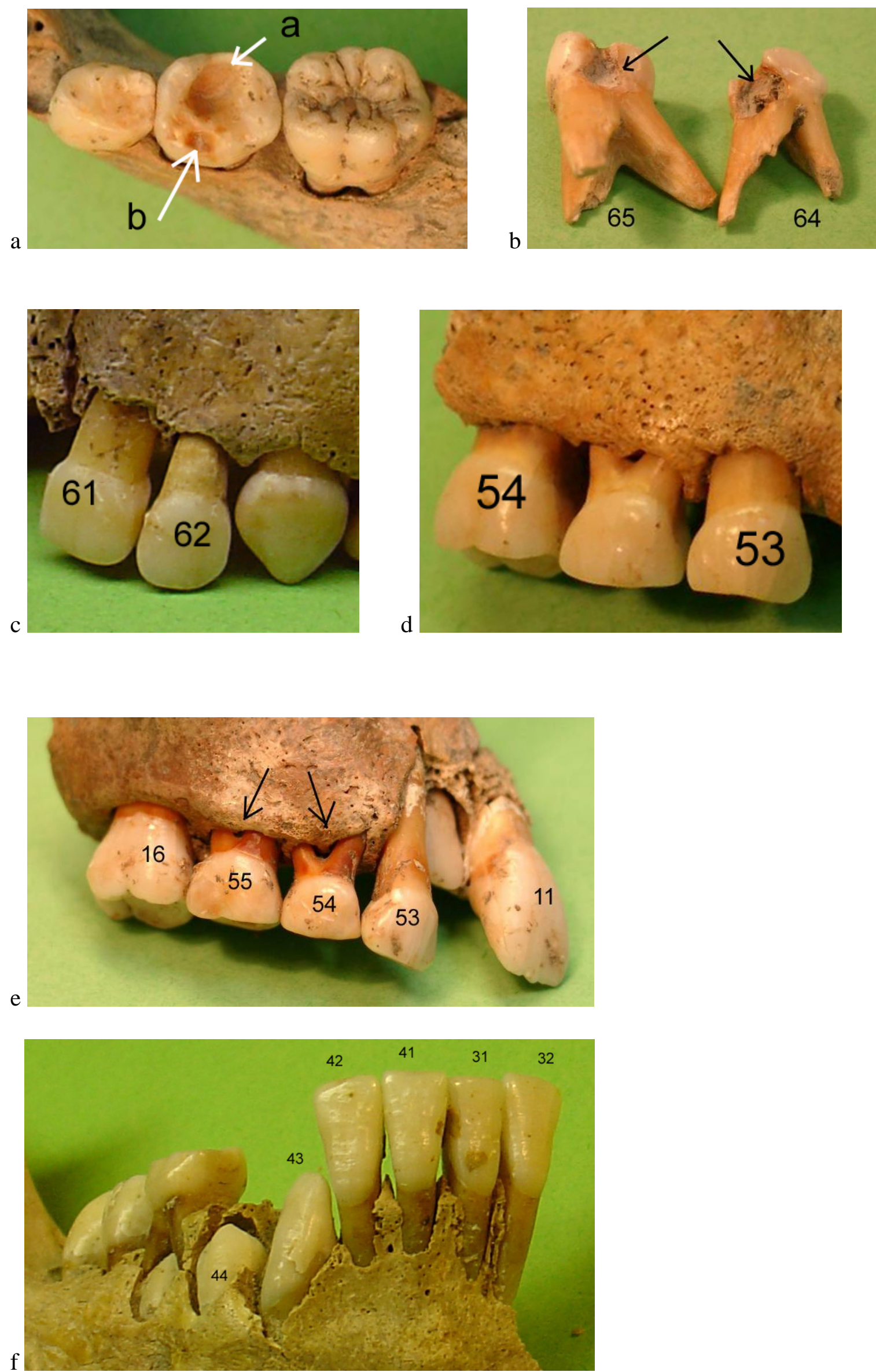
Abb. 20 a: Grab 220; 9,5 bis 12,5 Jahre. Geschlecht weiblich. Mandibula. Detailaufnahme aus Abb. 19 f. Transversale Schmelzhypoplasien an den Zähnen 31, 32, 41, 42 und 43 sowie Reste von Zahnstein an der labialen Fläche des Zahnes 31 sowie mesial an den Zähnen 31, 41 und 42.

Abb. 20 b: Grab 102; 9 bis 10,5 Jahre. Geschlecht nicht bestimmbar. Labialansicht des Zahnes 11. Im Bereich der Zahnwurzel befinden sich linienförmige Einziehungen als Zeichen von transversalen Zementhypoplasien.

Abb. 20 c: Grab 318; 7,5 bis 10 Jahre. Geschlecht wahrscheinlich männlich. Lingualansicht der linken Mandibula. Reste eines höhergradigen Zahnsteinbefalls $(\rightarrow)$ sind an den Zähnen 42, 32 und 36 zu erkennen. Der Knochenschwund im Bereich der Wurzel des Zahnes 36 ist das Zeichen eines apikalen Prozesses. Es bestand ein Abszess im Bereich von Zahnhals und -wurzel.

Abb. 20 d: Grab 318; 7,5 bis 10 Jahre. Geschlecht wahrscheinlich männlich. Buccalansicht der linken Mandibula, Detailaufnahme der Umgebung des Zahnes 36. Neben Zahnsteinresten an der Krone des Zahnes 36 (Z) ist die Reduktion des Alveolarknochens im Bereich der Wurzel von $36(\rightarrow)$ deutlich zu erkennen. Diese liegt etwa zur Hälfte frei. Das Kind litt an einem apikalen Prozess.

Abb. 20 e: Grab 102; 9 bis 10,5 Jahre. Geschlecht nicht bestimmbar. Distalansicht des Zahnes 11. Im Bereich der Zahnwurzel befinden sich feine Rillen als Zeichen transversaler Zementhypoplasien. 
Tafel 20
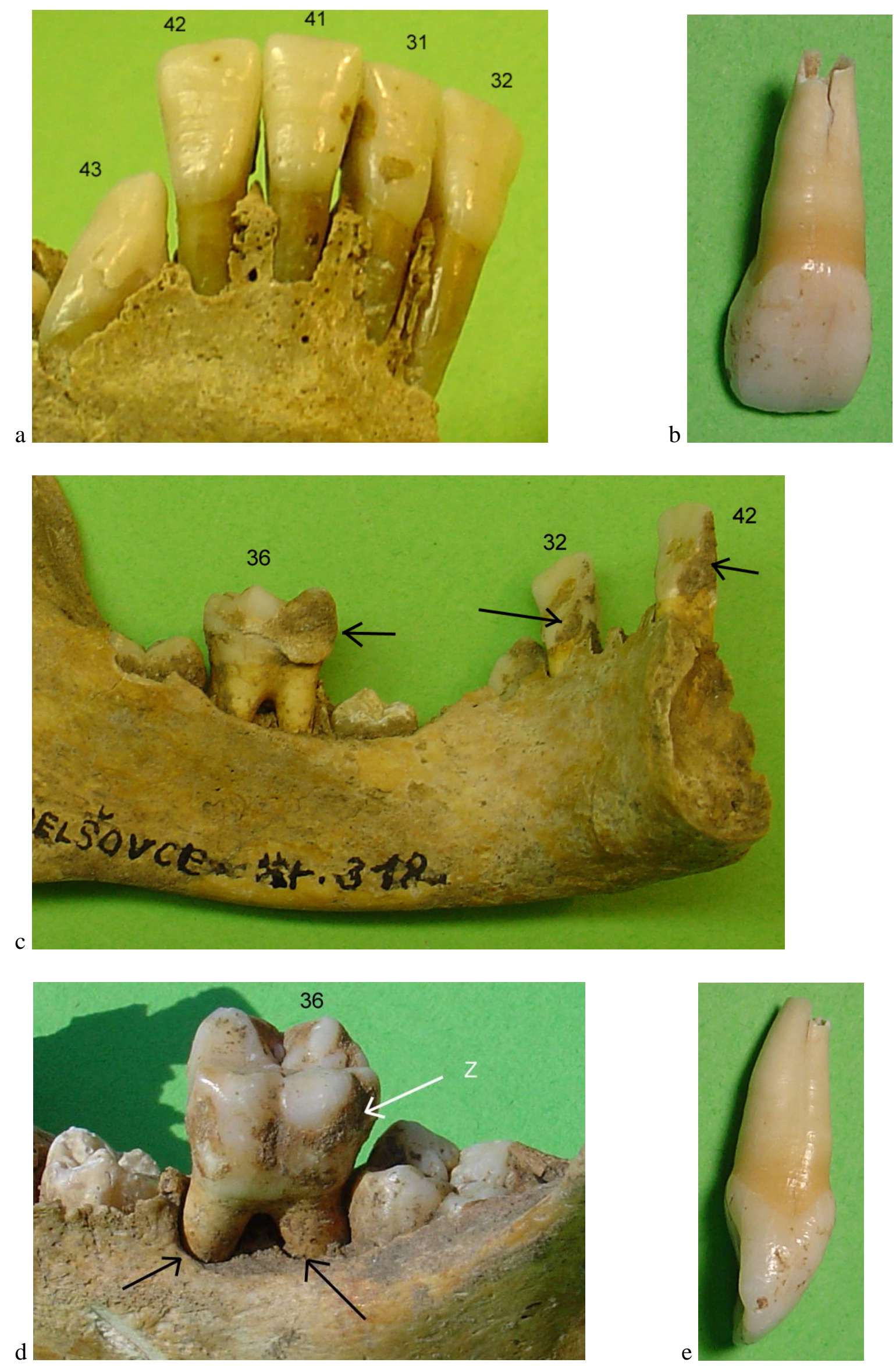
Abb. 21 a und b: Grab 65 A; 3 bis 4 Jahre. Geschlecht unbestimmbar. Zwei Fragmente des linken Humerus. Auf der Oberfläche zeigen sich plattenartige, poröse Auflagerungen $(\rightarrow)$.

Abb. 21 c: Grab 65 A; 3 bis 4 Jahre. Geschlecht unbestimmbar. Betrachtung eines Knochendünnschliffs $(50 \mu \mathrm{m})$ aus einem Fragment des linken Humerus (Abb. 21 a) im polarisierten Durchlicht unter Benutzung eines Hilfsobjekts Rot 1. Ordnung (Quarz) als Kompensator. Vergrößerung 25fach.

Auf der Compacta ist eine plattenartige Auflagerung zu erkennen.

a: Markraum, b: Außenseite, $\rightarrow$ : Auflagerung.

Abb. 21 d: Grab 65 A; 3 bis 4 Jahre. Geschlecht unbestimmbar. Betrachtung eines Knochendünnschliffs $(50 \mu \mathrm{m})$ aus einem Fragment des linken Humerus (Abb. 21 a) im polarisierten Durchlicht unter Benutzung eines Hilfsobjekts Rot 1. Ordnung (Quarz) als Kompensator. Detail aus Abb. 21 c. Vergrößerung 100fach.

Der ehemaligen Oberfläche (a) mit intakter äußerer Lamelle ist eine hämorrhagisch bedingte Knochenneubildung aufgelagert.

a: ehemalige Oberfläche, b: Auflagerung.

Abb. 21 e: Grab 158A; 7 bis 10 Jahre. Geschlecht unbestimmbar. Betrachtung eines Knochendünnschliffs $(70 \mu \mathrm{m})$ aus der rechten Ulna quer durch das Olecranon. Dünnschliffübersicht. Vergrößerung 4fach

An der Facies lateralis ist eine Auflagerung zu sehen $(\rightarrow)$.

Abb. 21 f: Grab 158A; 7 bis 10 Jahre. Geschlecht unbestimmbar. Betrachtung eines Knochendünnschliffs $(70 \mu \mathrm{m})$ aus der rechten Ulna quer durch das Olecranon im polarisierten Durchlicht unter Benutzung eines Hilfsobjekts Rot 1. Ordnung (Quarz) als Kompensator.

Vergrößerung 100fach.

Auf der alten äußeren Generallamelle (+) befindet sich eine brückenartige Lage neu gebildeten Knochens $(\rightarrow)$. 
Tafel 21
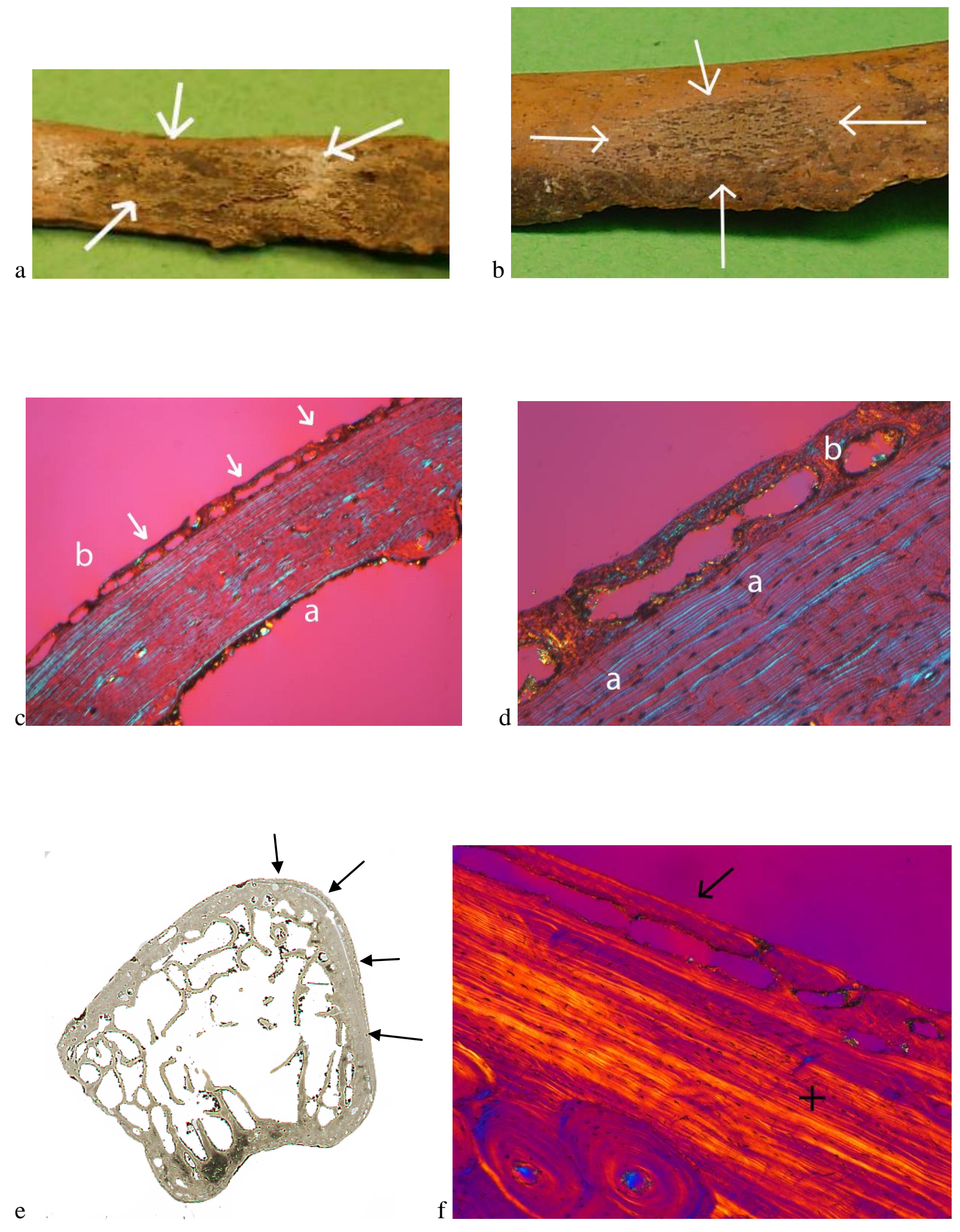
Abb. 22 a: Grab 99; 6 bis 8 Jahre. Geschlecht unbestimmbar. Linke Ulna, mittlerer Diaphyseabschnitt. Auf der Facies anterior ist eine langgezogene plattenartige Auflagerung zu sehen $(\rightarrow)$.

Abb. 22 b: Grab 99; 6 bis 8 Jahre. Geschlecht unbestimmbar. Betrachtung eines Knochendünnschliffs $(50 \mu \mathrm{m})$ aus der linken Ulna im Bereich der Auflagerung im polarisierten Durchlicht unter Benutzung eines Hilfsobjekts Rot 1. Ordnung (Quarz) als Kompensator. Vergrößerung 25 fach.

Es zeigt sich eine ehemals subperiostal gelegene, hämorrhagisch bedingte brückenartige Auflagerung $(\rightarrow)$.

Abb. 22 c: Grab 99; 6 bis 8 Jahre. Geschlecht unbestimmbar. Betrachtung eines Knochendünnschliffs $(70 \mu \mathrm{m})$ aus der linken Ulna im Bereich der Auflagerung im polarisierten Durchlicht unter Benutzung eines Hilfsobjekts Rot 1. Ordnung (Quarz) als Kompensator. Vergrößerung 100fach.

Im Randbereich der Auflagerung sind als Reste dieser Auflagerung „Füßchen“ zu erkennen.

Abb. 22 d: Grab 279A; 7 bis 9 Jahre. Geschlecht unbestimmbar. Betrachtung eines Knochendünnschliffs $(70 \mu \mathrm{m})$ aus der rechten Ulna im Übergang des mittleren Drittels in Richtung auf das Olecranon. Dünnschliffübersicht. Vergrößerung 4fach.

Beidseits des Margo interosseus befinden sich massive Auflagerungen auf den Facies anterior und posterior $(\rightarrow)$.

Abb. 22 e und f: Grab 279 A; 7 bis 9 Jahre. Geschlecht unbestimmbar. Betrachtung eines Knochendünnschliffs $(70 \mu \mathrm{m})$ aus der rechten Ulna im Übergang des mittleren Drittels zum Olecranon im polarisierten Durchlicht (Abb. 22 e) bzw. im polarisierten Durchlicht unter Benutzung eines Hilfsobjekts Rot 1. Ordnung (Quarz) als Kompensator (Abb. 22 f). Detail aus Abb. 22 d. Vergrößerung 25fach.

Die Knochenneubildung liegt auf der äußeren Generallamelle. Es handelt sich um eine hämorrhagisch bedingte Veränderung.

+: alte Oberfläche. 
Tafel 22
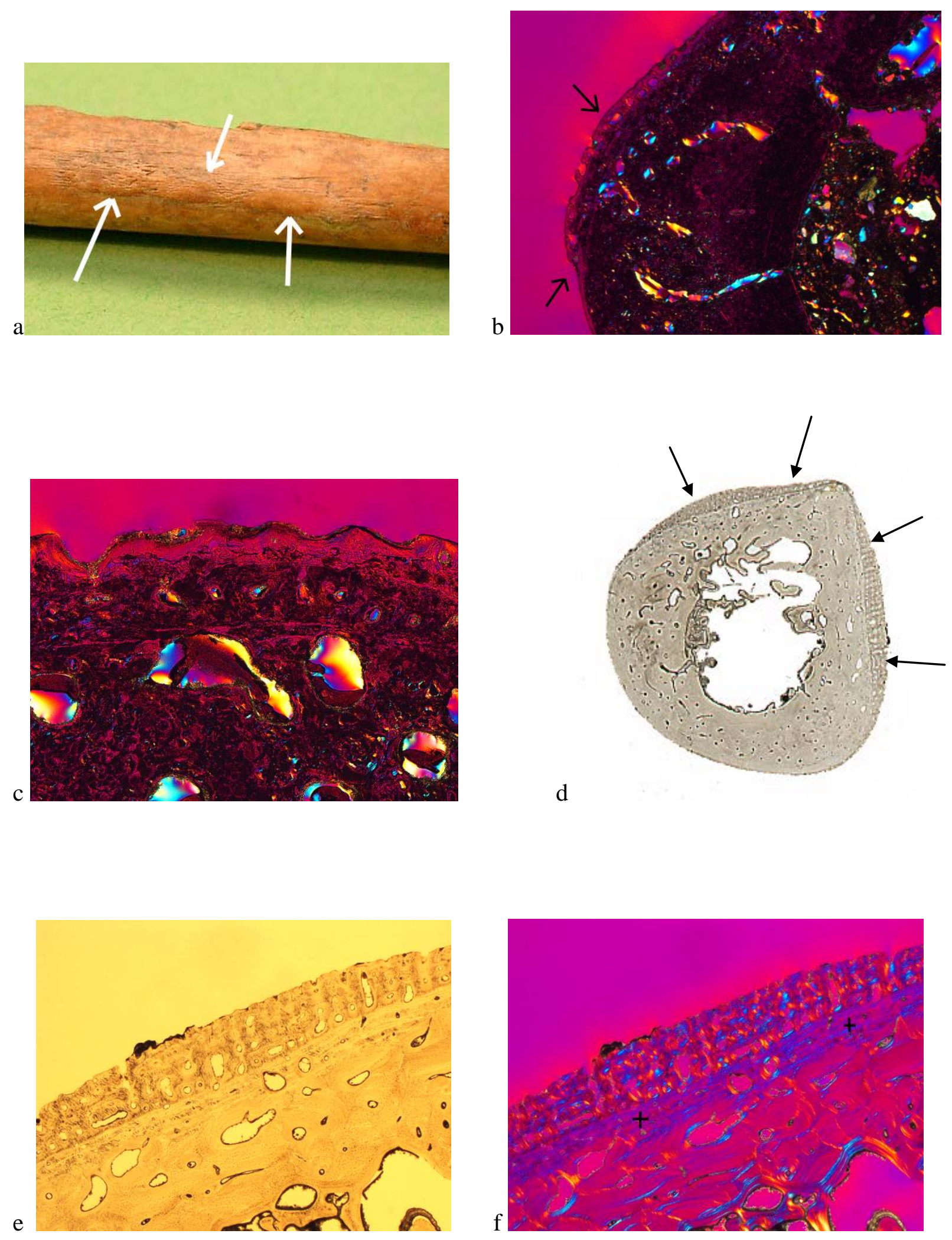
Abb. 23 a und b: Grab 279 A; 7 bis 9 Jahre. Geschlecht unbestimmbar. Betrachtung eines Knochendünnschliffs $(70 \mu \mathrm{m})$ aus der rechten Ulna (mittlerer Diaphysenabschnitt, Facies posterior) im polarisierten Durchlicht (Abb. 23 a) bzw. im polarisierten Durchlicht unter Benutzung eines Hilfsobjekts Rot 1. Ordnung (Quarz) als Kompensator (Abb. 23 b). Vergrößerung 25fach.

Der äußeren Generallamelle ist eine hämorrhagisch bedingte Knochenneubildung aufgelagert.

Abb. 23 c: Grab 279 A; 7 bis 9 Jahre. Geschlecht unbestimmbar. Betrachtung eines Knochendünnschliffs $(70 \mu \mathrm{m})$ aus der rechten Ulna (mittlerer Diaphysenabschnitt, Facies posterior) im polarisierten Durchlicht unter Benutzung eines Hilfsobjekts Rot 1. Ordnung (Quarz) als Kompensator. Detail aus Abb. 22 f. Vergrößerung 100fach.

Die Auflagerung liegt als Zeichen der hämorrhagischen Genese der alten Oberfläche auf.

+: ehemalige Oberfläche.

Abb. 23 d: Grab 279 A; 7 bis 9 Jahre. Geschlecht unbestimmbar. Betrachtung eines Knochendünnschliffs $(70 \mu \mathrm{m})$ aus der rechten Ulna (mittlerer Diaphysenabschnitt, Facies posterior) im polarisierten Durchlicht unter Benutzung eines Hilfsobjekts Rot 1. Ordnung (Quarz) als Kompensator. Detail aus Abb. 23 b. Vergrößerung 100fach.

Die Knochenneubildung ist als Zeichen ihres hämorrhagischen Ursprungs der äußeren Generallamelle aufgelagert.

+: alte Oberfläche.

Abb. 23 e: Grab 65A: 3 bis 4 Jahre. Geschlecht unbestimmbar. Palmaransicht des linken Os metacarpale III. Um ein Foramen nutricium herum befindet sich eine plattenartige Auflagerung $(\rightarrow)$ an der Palmarseite des dritten Mittelhandknochens der linken Seite.

Abb. 23 f: Grab 65A: 3 bis 4 Jahre. Geschlecht unbestimmbar. Lateralansicht des rechten Os ilii. In der Nähe des Acetabulum befindet sich eine plattenartige Auflagerung $(\rightarrow)$. 
Tafel 23
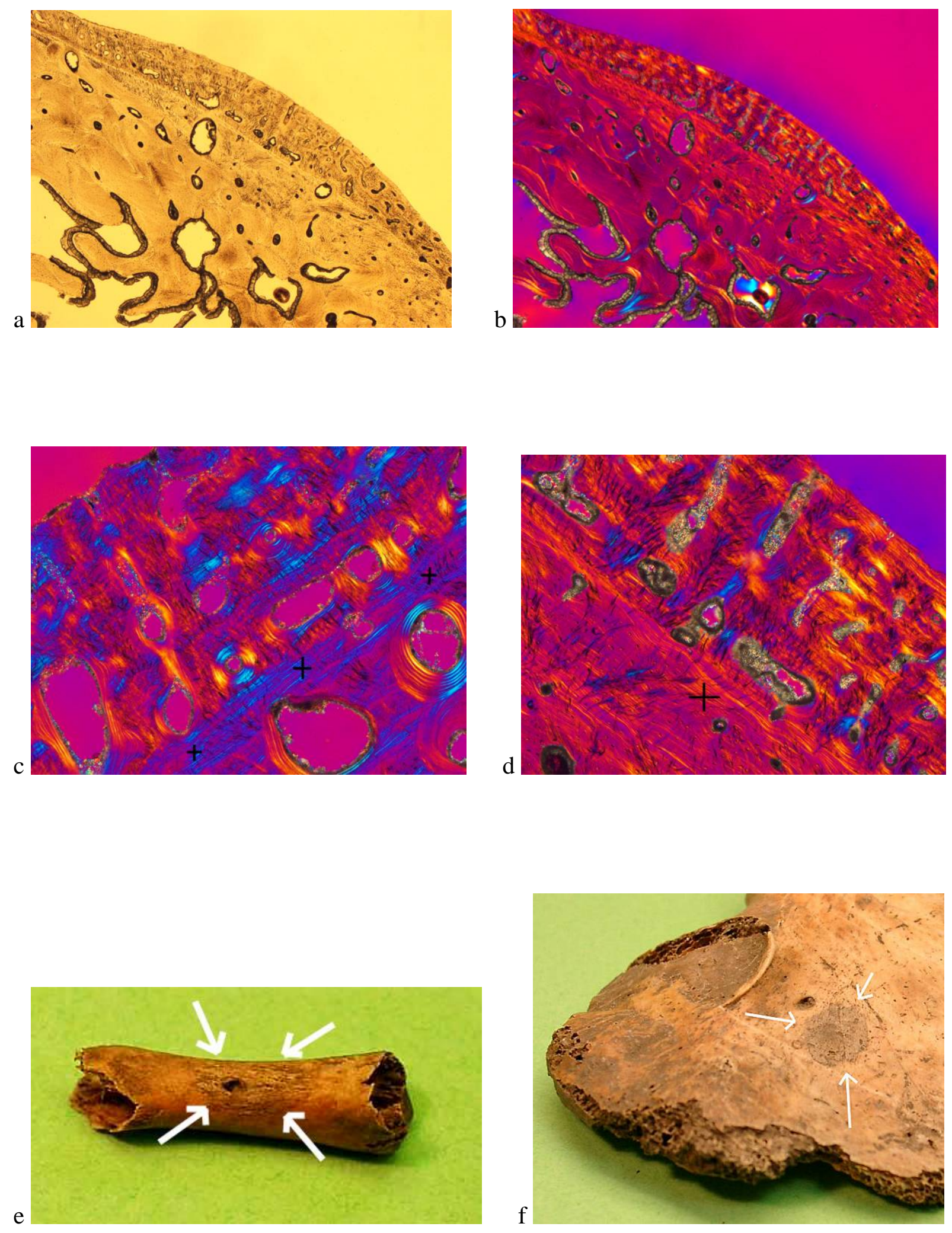
Abb. 24 a: Grab 158 A; 7 bis 10 Jahre. Geschlecht unbestimmbar. Rippenfragment, distales Drittel des Corpus. Blick auf die Innenfläche. Es zeigt sich eine muldenförmige Vertiefung $(\rightarrow)$

Abb. 24 b: Grab 158 A; 7 bis 10 Jahre. Geschlecht unbestimmbar. Rasterelektronenmikroskopische Aufnahme des Rippenfragments im Bereich der Mulde an der Innenfläche. Die deutlichen postmortalen Veränderungen zeigen, dass es sich um eine Pseudopathologie handelt.

$\rightarrow$ : muldenförmige Vertiefung, a: postmortal zerstörte Oberfläche. Vergrößerung $90 \mathrm{fach}$.

Abb. 24 c: Grab 279 A; 7 bis 9 Jahre. Geschlecht unbestimmbar. Ventralansicht des Sternums. Die Synchondrosis sternalis (b) ist atypisch zwischen das erste (f) und zweite (g) Corpussegment verlagert. Außerdem kam es zu einem frühzeitigen Verschluss der Synchondrosis manubriosternalis (---).

a: Incisura costalis II, b: Lage der Synchondrosis sterni, c: Incisura costalis I, d: Incisura jugularis, e: Manubrium sterni, f: erstes Segment des Corpus sterni, g: zweites Segment des Corpus sterni, ----: verknöcherte Synchondrosis manubriosternalis 
Tafel 24
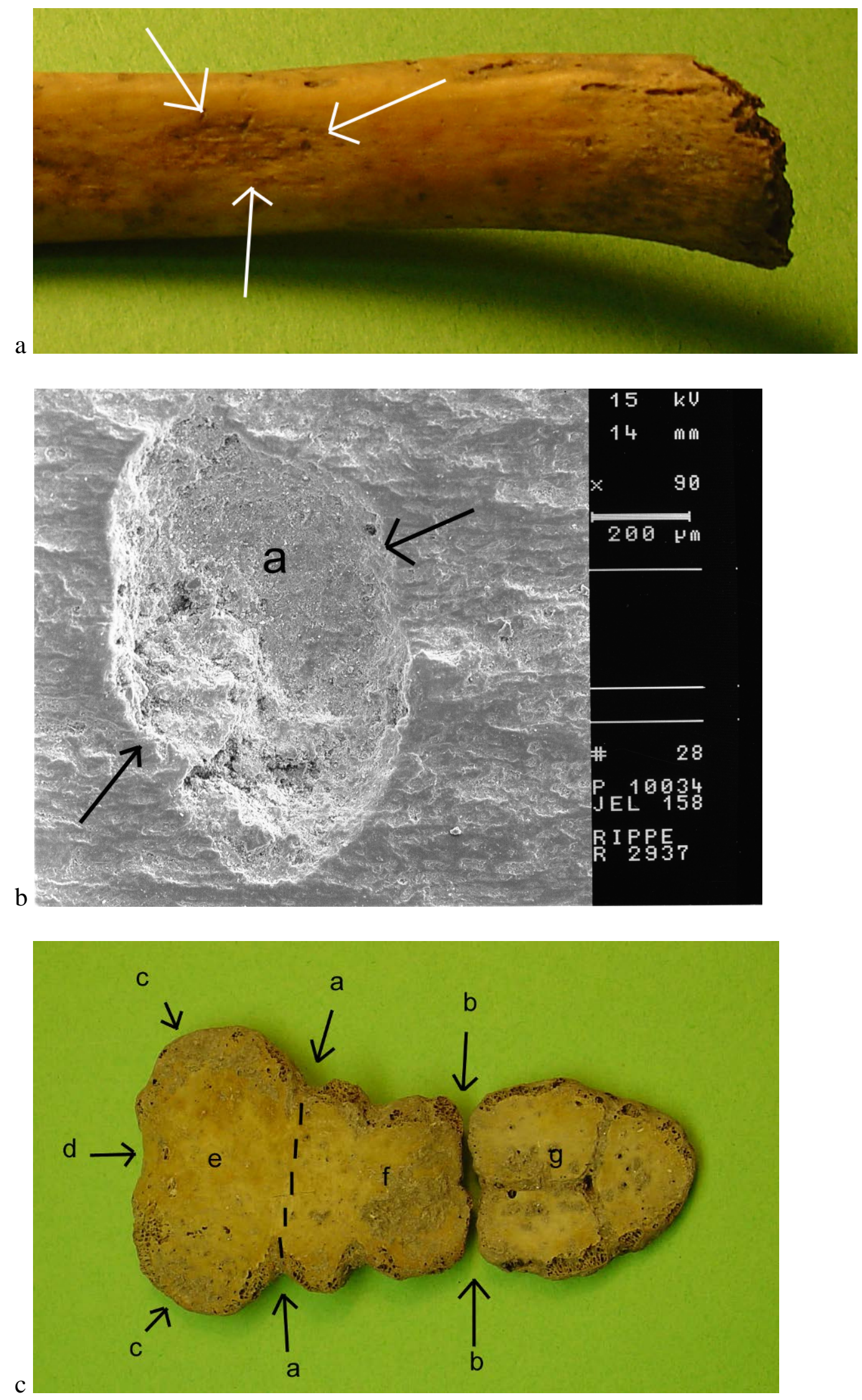
Abb. 25 a: Grab 350; 7 bis 9 Jahre. Geschlecht unbestimmbar. Dorsalansicht der Halswirbel C5 und C6. Die Wirbelbögen sind auf der rechten Seite ankylosiert (a). Der 6. Halswirbel weist eine Spaltbildung des Processus spinosus auf (b) (Spina bifida).

I: 5. Halswirbel, II: 6. Halswirbel, a: ankylosierte Wirbelbögen, b: gespaltener Processus spinosus des 6. Halswirbels, c: rechter Processus articularis superior des 5. Halswirbels

Abb. 25 b: Grab 350; 7 bis 9 Jahre. Geschlecht unbestimmbar. Dorsalansicht der Halswirbel C5 bis C7. Die Wirbelbögen von C5 und C6 sind auf der rechten Seite ankylosiert (a). Der Processus spinosus des 6. Halswirbels ist vollständig gespalten. Der 7. Halswirbel weist eine aberrante Form der Spaltbildung auf.

I: 5. Halswirbel, II: 6. Halswirbel, a: ankylosierte Wirbelbögen, b: verzogener Processus spinosus des 7. Halswirbels mit aberranter Spaltbildung.

Abb. 25 c: Grab 350; 7 bis 9 Jahre. Geschlecht unbestimmbar. Ansicht von schräg rechts lateral auf die Halswirbel C5 bis C7. Die Wirbelbögen des 5. Und 6. Halswirbels sind ankylosiert $(\leftrightarrow)$.

I: 5. Halswirbel, II: 6. Halswirbel, a: gespaltener und verzogener Processus spinosus des 6. Halswirbels, $\leftrightarrow:$ ankylosierte Wirbelbögen von C5 und C6. 
Tafel 25
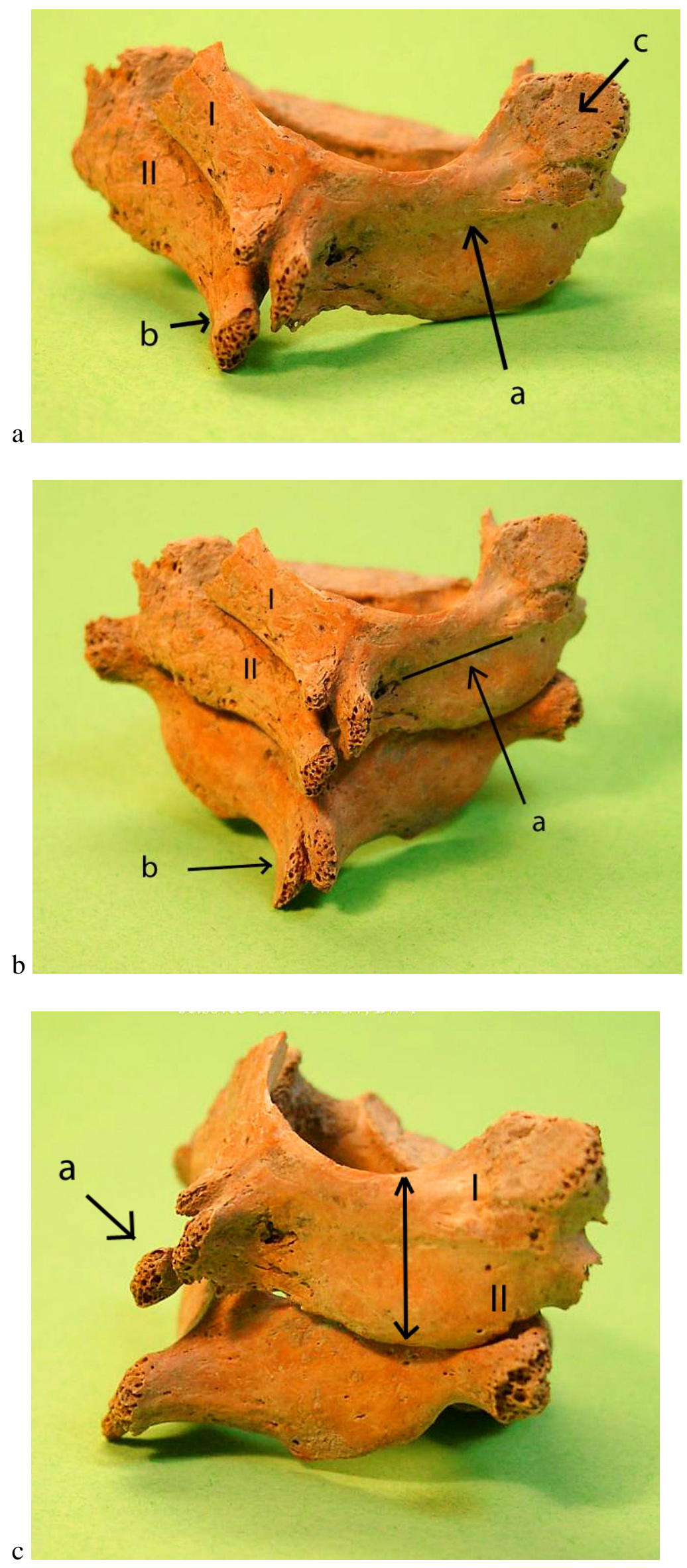
Tafel 26:

Abb. 26 a: Grab 126; 9 bis 11 Jahre. Geschlecht weiblich. Caudalansicht des dritten Sakralwirbels. Als Zeichen einer Spina bifida weist der Processus spinosus eine Spaltbildung auf.

a: Pars lateralis links, b: Pars lateralis rechts, c: ventrale Seite, $\rightarrow$ Spaltung des Processus spinosus.

Abb. 26 b: Grab 126; 9 bis 11 Jahre. Geschlecht weiblich. Dorsalansicht des zweiten und dritten Sakralwirbels. Der dritte Sakralwirbel weist eine Spaltbildung des Processus spinosus auf.

a: zweiter Sakralwirbel, b: dritter Sakralwirbel, $\rightarrow$ Spaltung des Processus spinosus.

Abb. 26 c: Grab 126; 9 bis 11 Jahre. Geschlecht weiblich. Ansicht auf drei Halswirbel von oben. Die Processus spinosi sind als Zeichen einer Skoliose nach rechts verzogen.

a: Processus transversi links, b: Processus spinosus, — verdrehter Achsverlauf der Processus spinosi.

Abb. 26 d: Grab 228 B; 6 bis 8 Jahre. Geschlecht weiblich. Dorsalansicht eines Lendenwirbels. Die Wirbelbögen sind in sich und gegeneinander verdreht. Es besteht der Verdacht auf eine Skoliose. 
Tafel 26
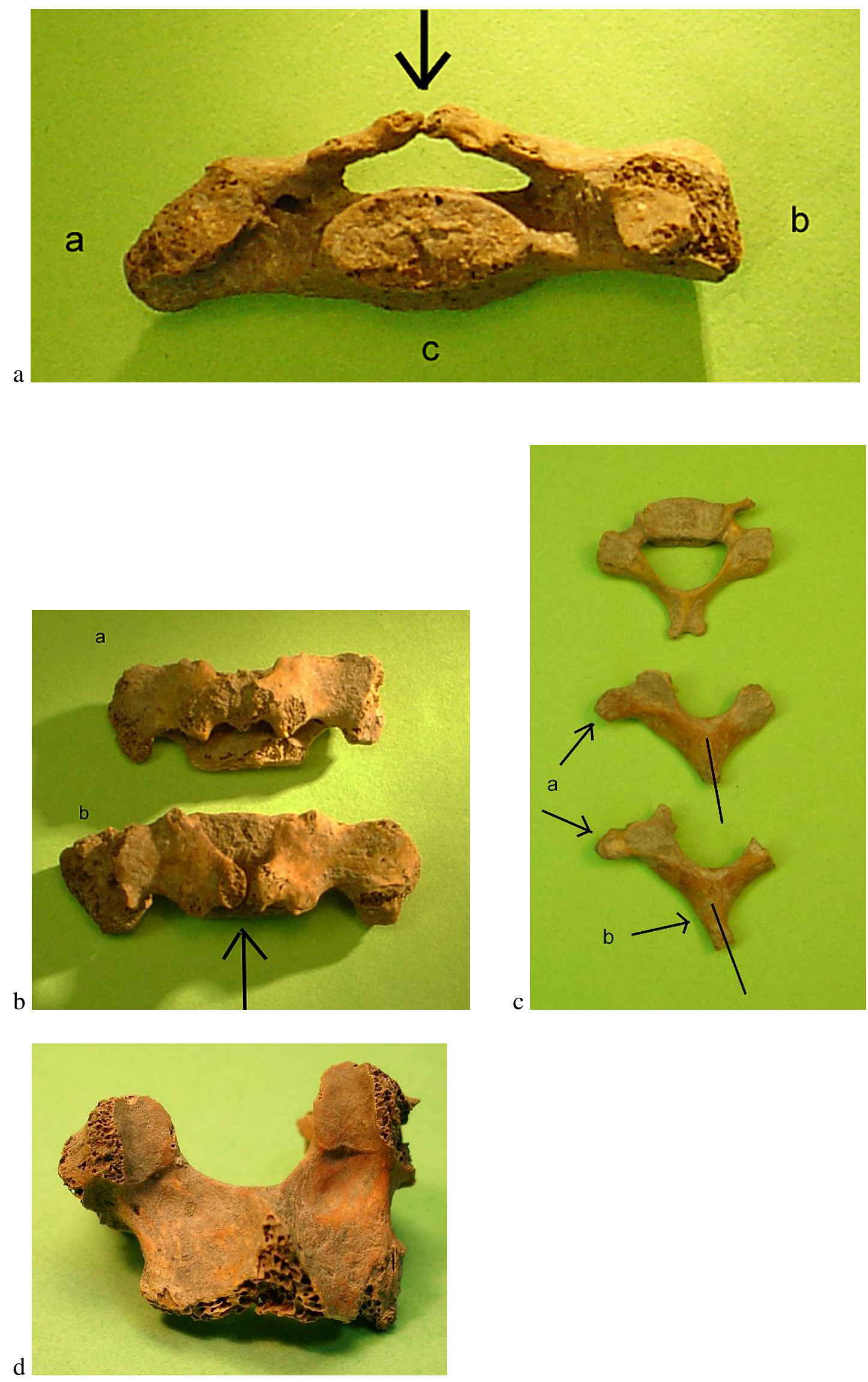
Abb. 27 a: Grab 299; 9 bis 10,5 Jahre. Geschlecht nicht bestimmbar. Os ischii rechts. Sicht auf Facies semilunata und Fossa acetabuli. Vermehrte Porosität im Bereich der Fossa acetabuli.

Abb. 27 b: Grab 299; 9 bis 10,5 Jahre. Geschlecht nicht bestimmbar. Os ischii rechts, Detail aus Abb. 27 a. Fossa acetabuli. Über die Norm verstärkte Porosität. 
Tafel 27
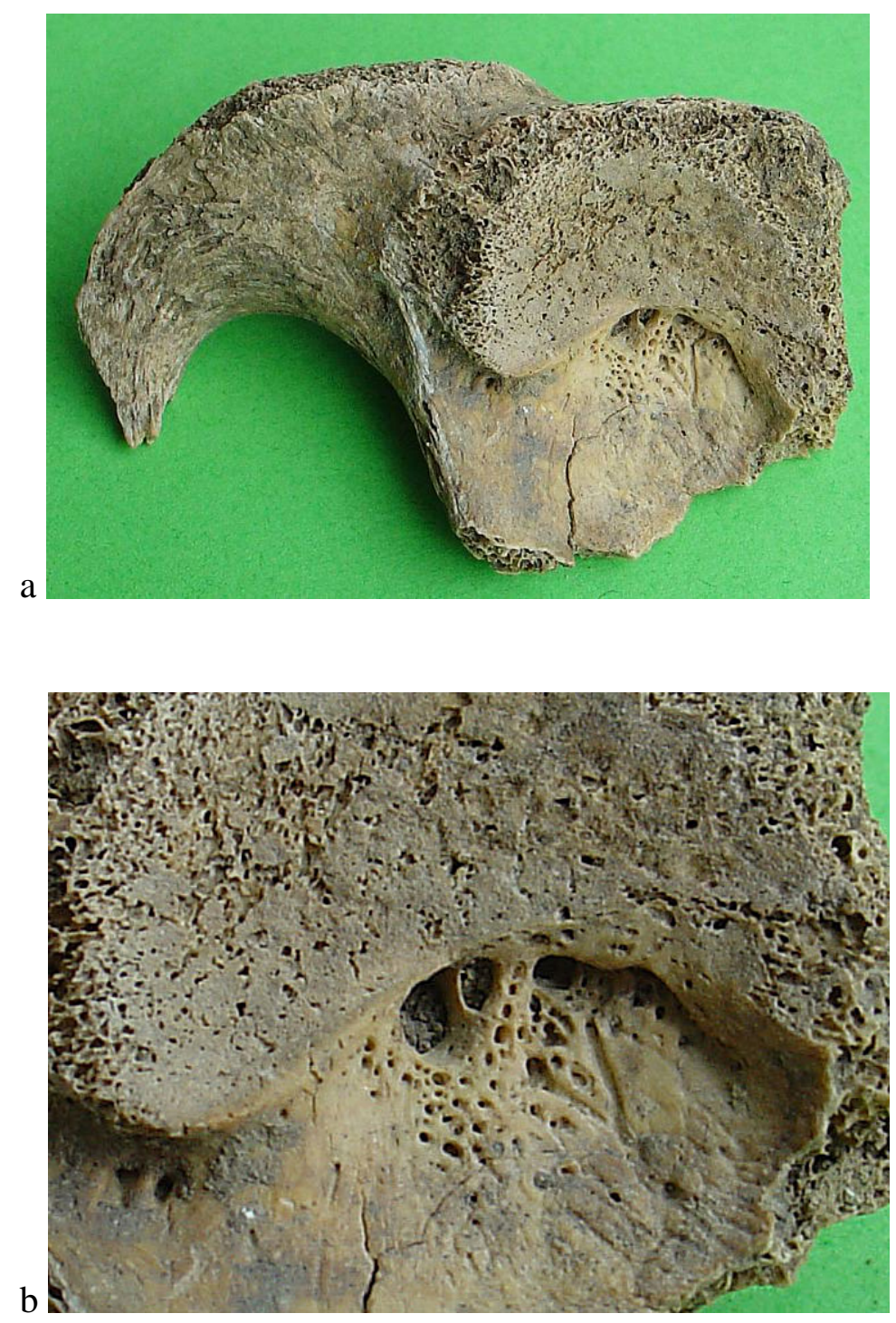
Tafel 28

Abb. 28 a: Grab 95; 3,5 bis 5,5 Jahre. Geschlecht unbestimmbar. Betrachtung eines Knochendünnschliffs $(50 \mu \mathrm{m})$ aus dem linken Femur im polarisierten Durchlicht unter Benutzung eines Hilfsobjekts Rot 1. Ordnung (Quarz) als Kompensator. Vergrößerung 25fach.

Die äußere Compacta zeigt einem Gürtel aus Resorptionshöhlen, der im Zusammenhang mit dem normalen appositionellen Wachstum zu sehen ist. Zeichen einer pathologisch bedingten Auflagerung sind nicht zu finden.

a: Compacta mit in Ketten angeordneten Resorptionshöhlen.

Abb. 28 b: Grab 95; 3,5 bis 5,5 Jahre. Geschlecht unbestimmbar. Betrachtung eines Knochendünnschliffs $(50 \mu \mathrm{m})$ aus dem linken Femur im polarisierten Durchlicht unter Benutzung eines Hilfsobjekts Rot 1. Ordnung (Quarz) als Kompensator. Detail aus Abb. 28 a. Vergrößerung 100fach.

Detail der Resorptionshöhlen in der äußeren Compacta.

*: Resorptionshöhlen.

Abb. 28 c: Grab 95; 3,5 bis 5,5 Jahre. Geschlecht unbestimmbar. Dorsalansicht der linken Tibia, mittleres Diaphysendrittel. Makroskopisch zeigt sich eine plattenartige Auflagerung $(\rightarrow)$.

Abb. 28 d: Grab 95; 3,5 bis 5,5 Jahre. Geschlecht unbestimmbar. Betrachtung eines Knochendünnschliffs $(50 \mu \mathrm{m})$ aus der linken Tibia im polarisierten Durchlicht unter Benutzung eines Hilfsobjekts Rot 1. Ordnung (Quarz) als Kompensator. Vergrößerung 25fach.

An der Facies posterior weist die äußere Compacta als Zeichen des normalen appositionellen Wachstums halbfertige, neugebildete Haverssystemen auf $(\rightarrow)$. Zeichen einer pathologisch bedingten Auflagerung sind nicht zu finden.

a: normal konfigurierte Compacta, $\rightarrow$ : halbfertige Haverssysteme.

Abb. 28 e: Grab 95; 3,5 bis 5,5 Jahre. Geschlecht unbestimmbar. Betrachtung eines Knochendünnschliffs $(50 \mu \mathrm{m})$ aus der linken Tibia im polarisierten Durchlicht unter Benutzung eines Hilfsobjekts Rot 1. Ordnung (Quarz) als Kompensator. Detail aus Abb. 28 d. Vergrößerung 100fach.

Es sind zwei halbfertige Haverssysteme erkennbar $(\rightarrow)$.

a: Compacta, $\rightarrow$ : halbfertige Haverssysteme. 
Tafel 28
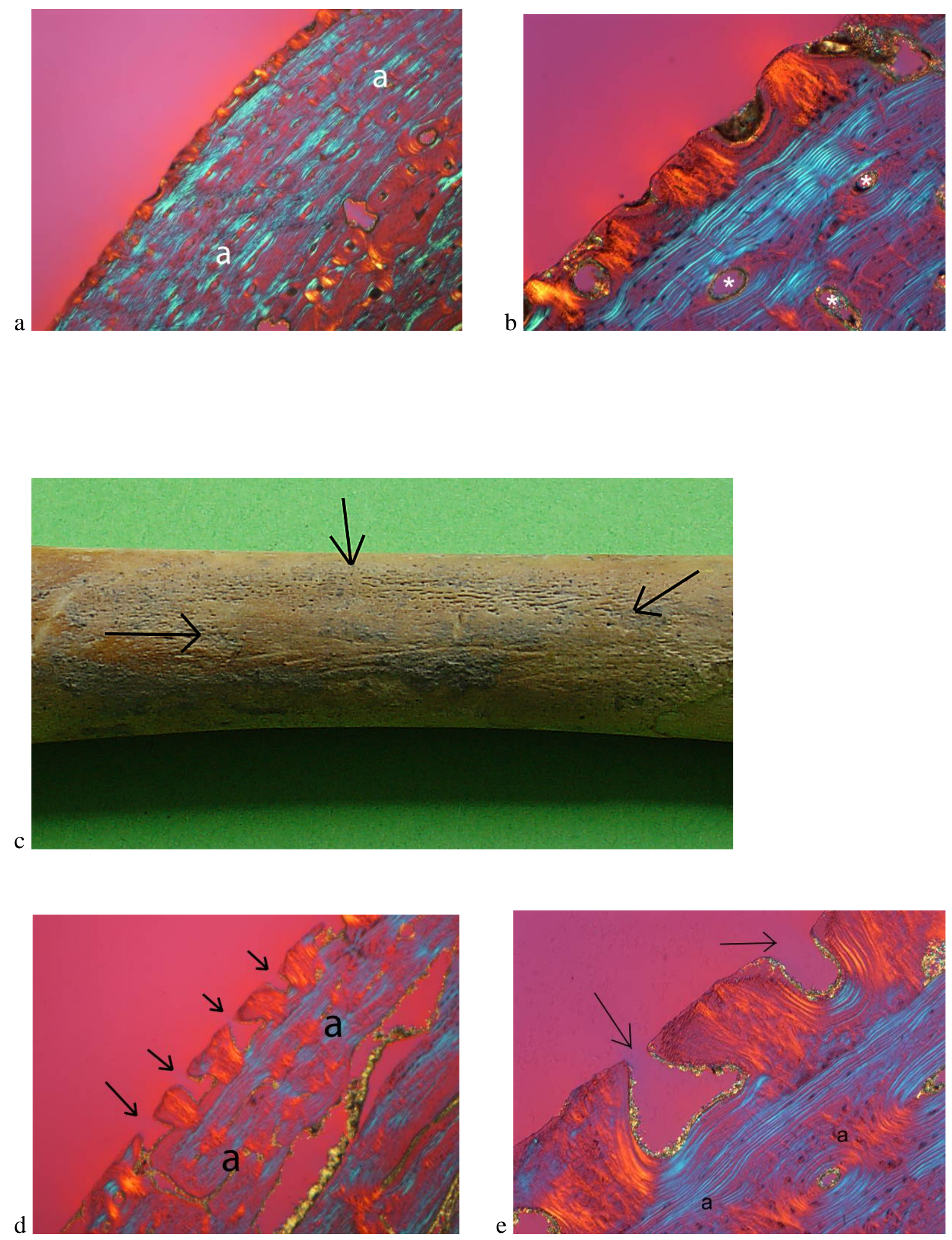
Abb. 29 a: Grab 334; 12 bis 14 Jahre. Geschlecht wahrscheinlich männlich. Betrachtung eines Knochendünnschliffs $(50 \mu \mathrm{m})$ aus der linken Tibia im mittleren Diaphysenabschnitt im polarisierten Durchlicht unter Benutzung eines Hilfsobjekts Rot 1. Ordnung (Quarz) als Kompensator. Vergrößerung 25fach.

An der Facies posterior sind Reste einer ehemals subperiostal gelegenen, hämorrhagisch bedingten Auflagerung erkennbar.

Abb. 29 b: Grab 334; 12 bis 14 Jahre. Geschlecht wahrscheinlich männlich. Betrachtung eines Knochendünnschliffs $(50 \mu \mathrm{m})$ aus der linken Tibia im polarisierten Durchlicht unter Benutzung eines Hilfsobjekts Rot 1. Ordnung (Quarz) als Kompensator. Vergrößerung 100fach.

Die Detailaufnahme der Auflagerung zeigt, dass diese der ehemalige Oberfläche $(+)$ aufgelagert ist, was auf den hämorrhagisch bedingten Ursprung der Auflagerung hinweist.

Abb. 29 c: Grab 604 B; 11 bis 14 Jahre. Geschlecht unbestimmbar. Betrachtung eines Knochendünnschliffs $(70 \mu \mathrm{m})$ aus dem linken Femur im polarisierten Durchlicht unter Benutzung eines Hilfsobjekts Rot 1. Ordnung (Quarz) als Kompensator. Vergrößerung 25fach.

Die Pfeile $(\rightarrow)$ zeigen auf die Reste einer hämorrhagisch bedingten Auflagerung.

Abb. 29 d: Grab 604 B; 11 bis 14 Jahre. Geschlecht unbestimmbar. Betrachtung eines Knochendünnschliffs $(70 \mu \mathrm{m})$ aus dem linken Femur im polarisierten Durchlicht unter Benutzung eines Hilfsobjekts Rot 1. Ordnung (Quarz) als Kompensator. Detail aus Abb. 29 c. Vergrößerung 100fach.

Reste einer hämorrhagisch bedingten Auflagerung $(\rightarrow)$ auf der ehemaligen Oberfläche $(+)$.

Abb. 29 e: Grab 220; 9,5 bis 12,5 Jahre. Geschlecht weiblich. Betrachtung eines Knochendünnschliffs $(50 \mu \mathrm{m})$ aus dem linken Femur (distal des Trochanter minor) im polarisierten Durchlicht unter Benutzung eines Hilfsobjekts Rot 1. Ordnung (Quarz) als Kompensator. Vergrößerung 25fach.

Reste einer hämorrhagisch bedingten Auflagerung (a) auf der ehemaligen Oberfläche (+). a: Auflagerung, b: postmortal zerstörte Oberfläche, +: äußere Generallamelle.

Abb. 29 f: Grab 220; 9,5 bis 12,5 Jahre. Geschlecht weiblich. Betrachtung eines Knochendünnschliffs $(50 \mu \mathrm{m})$ aus dem linken Femur im polarisierten Durchlicht unter Benutzung eines Hilfsobjekts Rot 1. Ordnung (Quarz) als Kompensator. Detail aus Abb. 29 e. Vergrößerung 100fach.

Reste einer hämorrhagisch bedingten Auflagerung auf der ehemaligen Oberfläche (+). 
Tafel 29
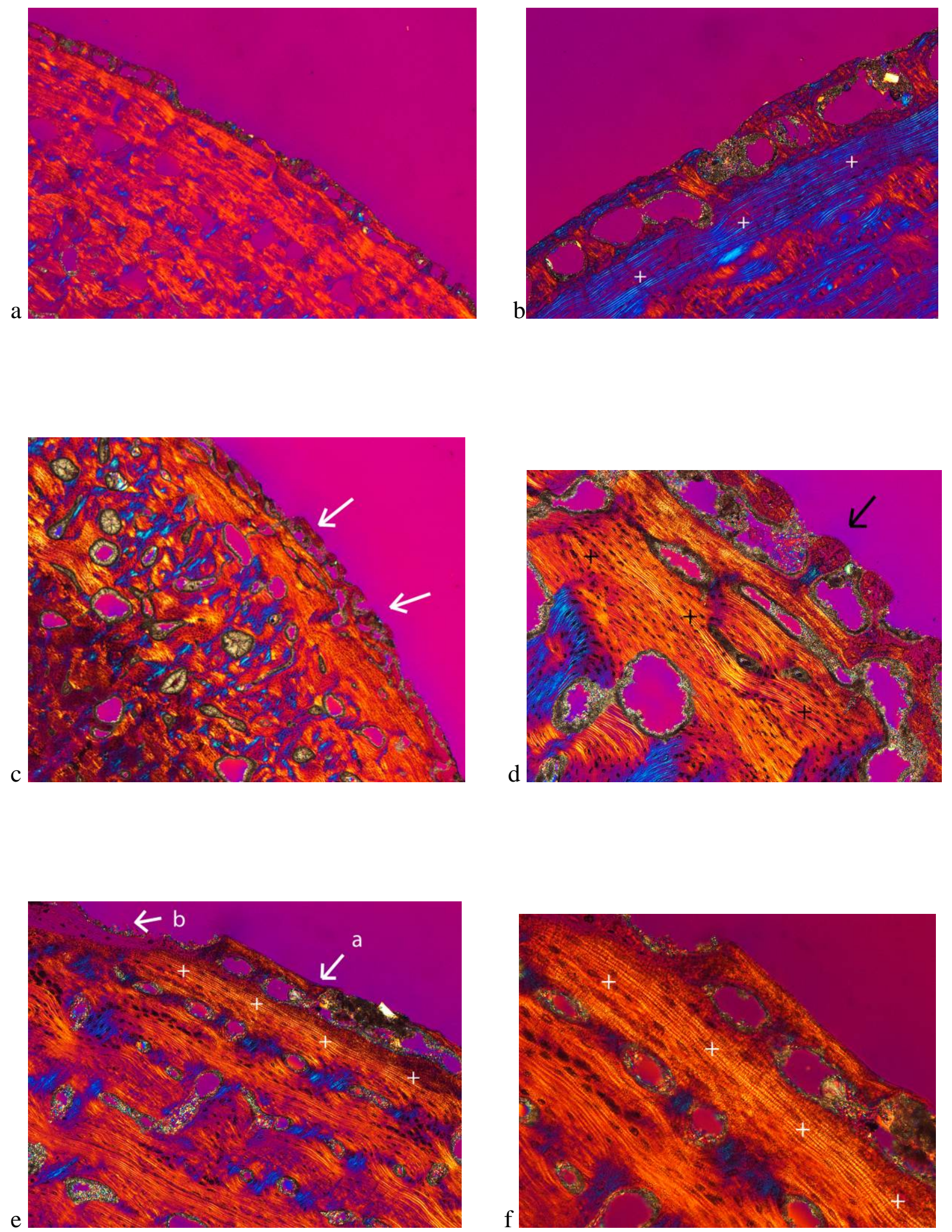
Abb. 30 a: Grab 169; 6 bis 7 Jahre. Geschlecht unbestimmbar. Distales Drittel des rechten Femurs, Facies dorsalis. Es ist eine längsorientierte, streifige Auflagerung $(\rightarrow$ ) erkennbar.

Abb. 30 b: Grab 169; 6 bis 7 Jahre. Geschlecht unbestimmbar. Proximales Drittel der rechten Fibula, Facies medialis. Es zeigt sich eine längsorientierte, streifige Auflagerung $(\rightarrow)$.

Abb. 30 c und d: Grab 169; 6 bis 7 Jahre. Geschlecht unbestimmbar. Betrachtung eines Knochendünnschliffs $(50 \mu \mathrm{m})$ aus dem rechten Femur im polarisierten Durchlicht (Abb. 30 c) bzw. im polarisierten Durchlicht unter Benutzung eines Hilfsobjekts Rot 1. Ordnung (Quarz) als Kompensator (Abb. 30 d). Vergrößerung 100fach.

Auf der Facies dorsalis kommt eine der äußeren Generallamelle aufgelagerte Knochenneubildung zur Darstellung. 
Tafel 30
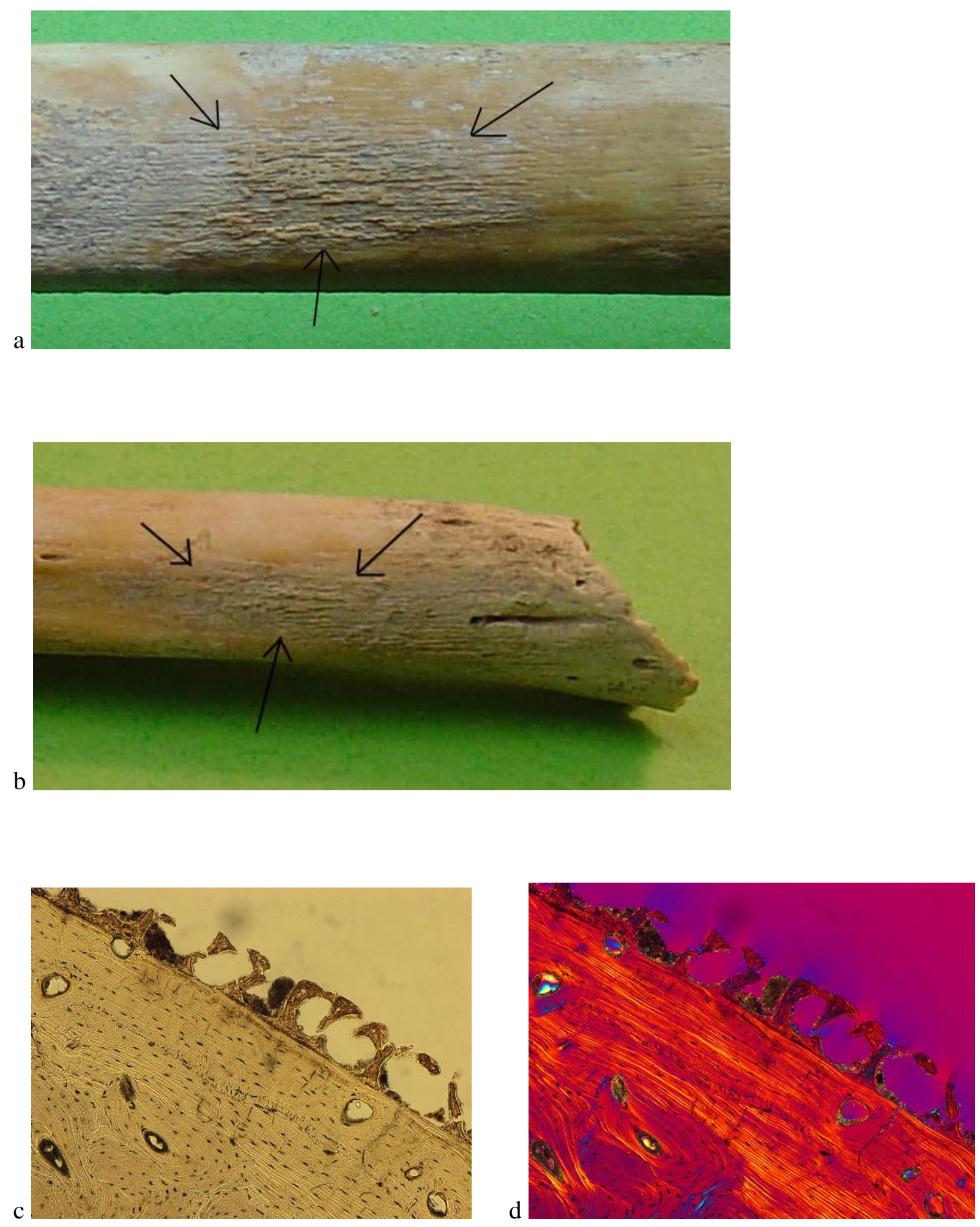
Abb. 31 a: Grab 254 B; 7 bis 10 Jahre. Geschlecht unbestimmbar. Distales Drittel des linken Femurs. Der Facies anterior ist großflächig eine plattenartige, poröse Knochenneubildung aufgelagert $(\rightarrow)$. Zum Teil ist diese postmortal abgemürbt.

Abb. 31 b und c: Grab 254 B; 7 bis 10 Jahre. Geschlecht unbestimmbar. Betrachtung eines Knochendünnschliffs $(70 \mu \mathrm{m})$ aus dem distalen Drittel des linken Femurs im polarisierten Durchlicht (Abb. 31 b) bzw. im polarisierten Durchlicht unter Benutzung eines Hilfsobjekts Rot 1. Ordnung (Quarz) als Kompensator (Abb. 31 c). Vergrößerung 25fach.

Die poröse Knochenneubildung in Bereich der Facies anterior ist der Compacta aufgelagert. Zusätzlich sind deutliche Spuren postmortaler Zerstörung zu erkennen.

Abb. 31d und e: Grab 254 B; 7 bis 10 Jahre. Geschlecht unbestimmbar. Betrachtung eines Knochendünnschliffs $(70 \mu \mathrm{m})$ aus dem linken Femur im polarisierten Durchlicht (Abb. $31 \mathrm{~d}$ ) bzw. im polarisierten Durchlicht unter Benutzung eines Hilfsobjekts Rot 1. Ordnung (Quarz) als Kompensator (Abb. 31 e). Detail aus 31 b. Vergrößerung 100fach.

Die der Compacta aufliegende Knochenneubildung ist vermutlich hämorrhagischen Ursprungs. Die Auflagerung weist deutliche Spuren postmortaler Zerstörung auf. 
Tafel 31
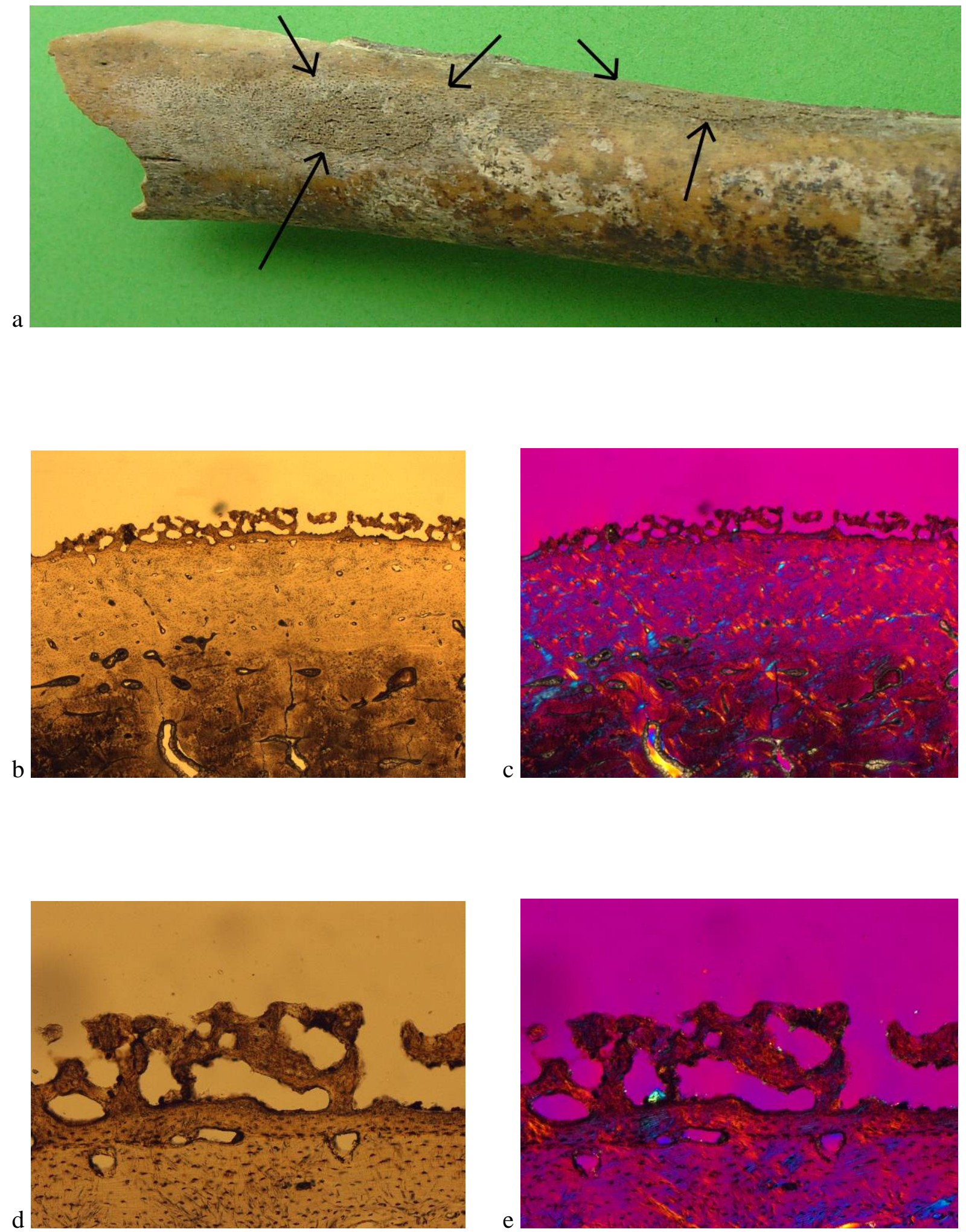
Abb. 32 a: Grab 254 B; 7 bis 10 Jahre. Lateralansicht der linken Tibia, proximales Drittel. Die Oberfläche ist lateral der Tuberositas tibiae längsgestreift.

a: streifig, poröse Oberfläche im Sulcus lateral der Tuberositas tibiae, b: postmortale Eröffnung von Gefäßkanälen, *: Tuberositas tibiae.

Abb. 32 b und c: Grab 254 B; 7 bis 10 Jahre. Geschlecht unbestimmbar. Betrachtung eines Knochendünnschliffs $(70 \mu \mathrm{m})$ aus der linken Tibia durch die Längsrillen lateral der Tuberositas tibiae im polarisierten Durchlicht (Abb. 32 b) bzw. im polarisierten Durchlicht unter Benutzung eines Hilfsobjekts Rot 1. Ordnung (Quarz) als Kompensator (Abb. 32 c). Vergrößerung 25fach.

Es sind die Reste einer postmortal abgemürbten, subperiostal gelegenen Auflagerung zu erkennen.

Abb. 32 d: Grab 254 B; 7 bis 10 Jahre. Geschlecht unbestimmbar. Betrachtung eines Knochendünnschliffs $(70 \mu \mathrm{m})$ aus der linken Tibia durch die Längsrillen lateral der Tuberositas tibiae im polarisierten Durchlicht unter Benutzung eines Hilfsobjekts Rot 1. Ordnung (Quarz) als Kompensator. Detail aus Abb. 32 c. Vergrößerung 100fach.

Die ehemals subperiostal gelegene Auflagerung ist teilweise postmortal abgemürbt.

Abb. 32 e: Grab 254 B; 7 bis 10 Jahre. Geschlecht unbestimmbar. Betrachtung eines Knochendünnschliffs $(70 \mu \mathrm{m})$ aus der linken Tibia durch die Längsrillen lateral der Tuberositas tibiae im polarisierten Durchlicht unter Benutzung eines Hilfsobjekts Rot 1. Ordnung (Quarz) als Kompensator. Detail aus Abb. 32 c. Vergrößerung 100fach.

Die ehemals subperiostal gelegene Auflagerung ist teilweise postmortal abgemürbt. 
Tafel 32
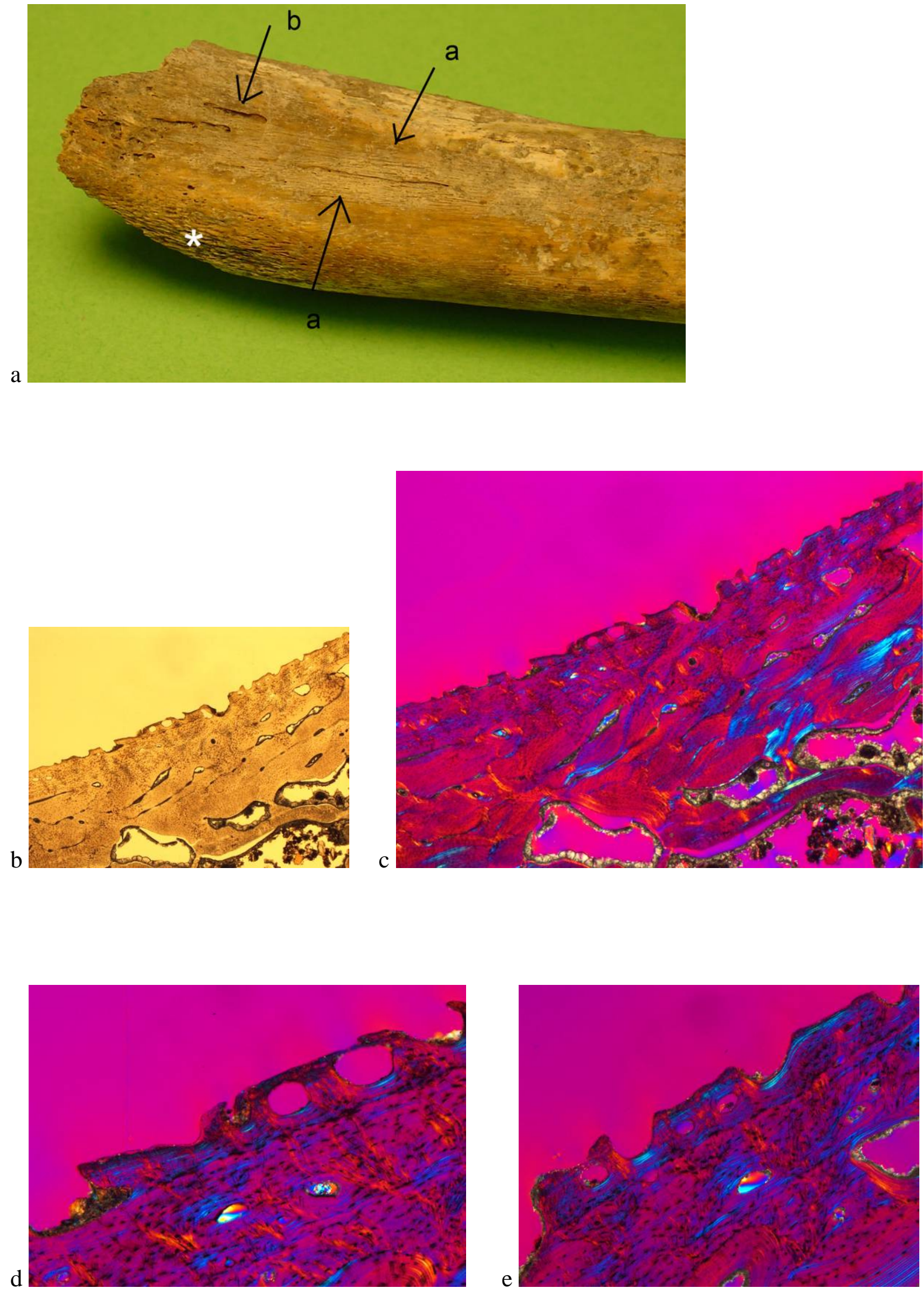
Abb. 33 a: Grab 103; 9 bis 13 Jahre. Geschlecht unbestimmbar. Dorsalansicht auf Caput und Collum des linken Femurs. Die Oberfläche im Bereich der craniodorsalen Collumfläche ist fein- bis mittelporös $(\rightarrow)$.

Abb. 33 b: Grab 103; 9 bis 13 Jahre. Geschlecht unbestimmbar. Rasterelektromemmikroskopische Aufnahme des Collums des linken Femurs. Größere intravital entstandene Foramina $\left(^{*}\right)$ sind von größtenteils postmortal entstandenen kleinen Foramina umgeben.

Vergrößerung 20fach.

Abb. 33 c: Grab 103; 9 bis 13 Jahre. Geschlecht unbestimmbar. Betrachtung eines Knochendünnschliffs $(50 \mu \mathrm{m})$ aus dem Collum des linken Femurs im polarisierten Durchlicht unter Benutzung eines Hilfsobjekts Rot 1. Ordnung (Quarz) als Kompensator. Vergrößerung 16fach.

Die Corticalis (a) der Dorsalseite ist durch zahlreiche Resorptionshöhlen stark aufgelockert. a: Corticalis, b: Markraum.

Abb. 33 d: Grab 103; 9 bis 13 Jahre. Geschlecht unbestimmbar. Betrachtung eines Knochendünnschliffs $(50 \mu \mathrm{m})$ aus dem Collum des linken Femurs im polarisierten Durchlicht unter Benutzung eines Hilfsobjekts Rot 1. Ordnung (Quarz) als Kompensator. Vergrößerung 16fach.

Die Corticalis (a) der ventralen Seite ist breiter als die der dorsalen (vgl. Abb. 33 c). Insgesamt sind weniger Resorptionshöhlen vorhanden.

a: Corticalis.

Abb. 33 e: Grab 103; 9 bis 13 Jahre. Geschlecht unbestimmbar. Betrachtung eines Knochendünnschliffs $(70 \mu \mathrm{m})$ aus dem Collum des linken Femurs im polarisierten Durchlicht unter Benutzung eines Hilfsobjekts Rot 1. Ordnung (Quarz) als Kompensator. Vergrößerung $100 f a c h$.

Detail der Resorptionshöhlen in der Corticalis der Dorsalseite. Trotz der starken postmortalen Versinterung sind Howshipsche Lakunen zu erkennen. Die Höhlen sind anders als bei normalen Resorptionsvorgängen im Rahmen des Wachstums nicht regelmäßig und glatt gewandet.

Abb. 33 f: Grab 103; 9 bis 13 Jahre. Geschlecht unbestimmbar. Betrachtung eines Knochendünnschliffs $(70 \mu \mathrm{m})$ aus dem Collum des linken Femurs im polarisierten Durchlicht unter Benutzung eines Hilfsobjekts Rot 1. Ordnung (Quarz) als Kompensator. Detail aus Abb. 33 e. Vergrößerung 200fach.

Am oberen Rand der Resorptionshöhle zeigen sich versinterte Howshipsche Lakunen als Zeichen einer vermehrten Resorption des Knochens. 
Tafel 33
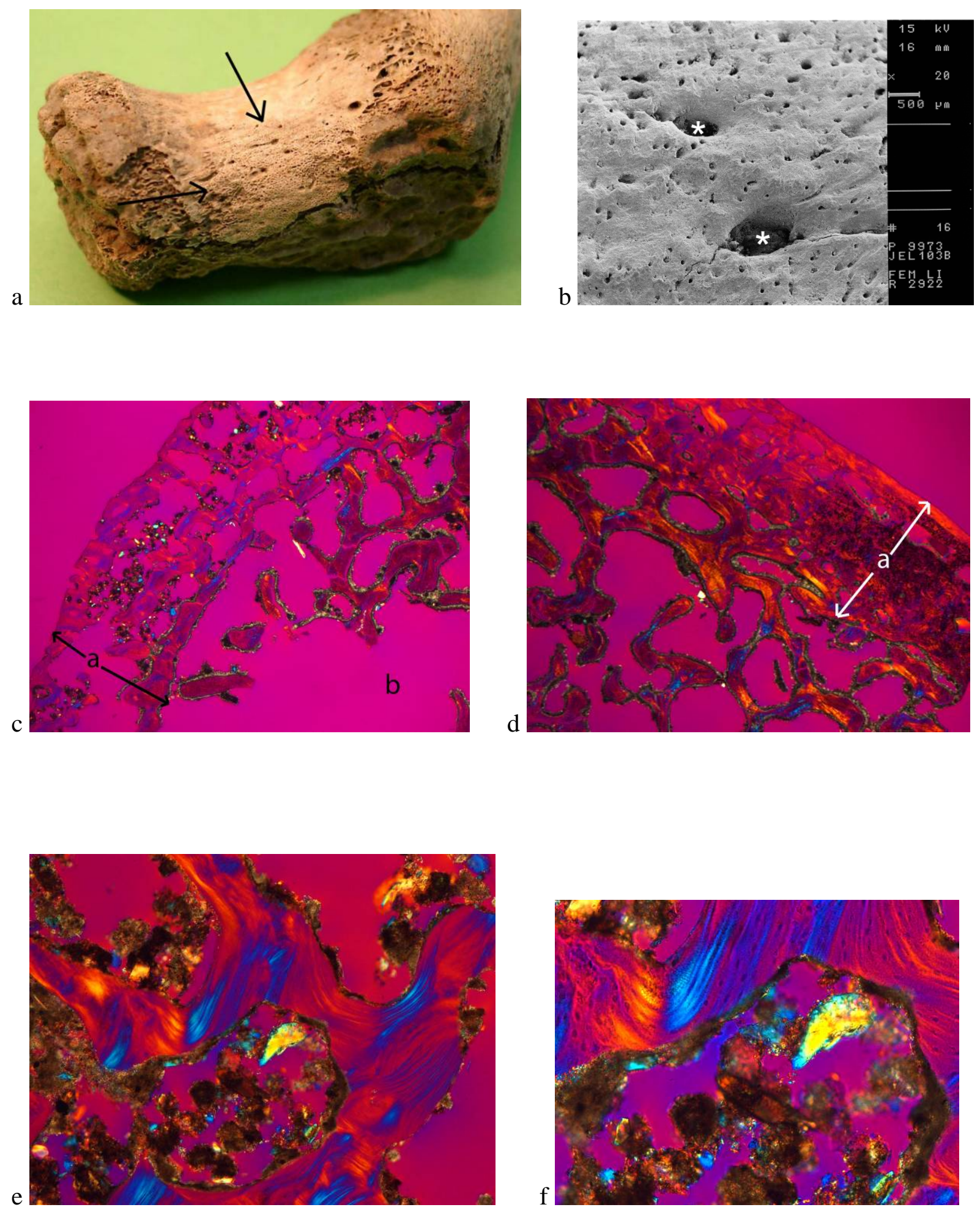
Abb. 34 a: Grab 566; 5,5 bis 7 Jahre. Geschlecht unbestimmbar. Dorsolateralansicht der linken Tibia. Der Sulcus musculi solei $(\rightarrow)$ ist sehr stark ausgeprägt und weist einen porösen Grund als Zeichen einer Überanspruchung des entspringenden Muskels (M. soleus) auf. a: Foramen nutricium, b: proximales Ende der Tibia, $\rightarrow$ : Sulcus musculi solei.

Abb. 34 b: Grab 135 A; 9 bis 11 Jahre. Geschlecht unbestimmbar. Dorsalansicht der rechten Tibia. Der Sulcus musculi solei $(\rightarrow)$ ist als Zeichen der Überanstrengung des entspringenden Muskels verbreitert.

Abb. 34 c: Grab 135 A; 9 bis 11 Jahre. Geschlecht unbestimmbar. Dorsalansicht des linken Femurs. Caudal des Collums befindet sich eine knotige Exostose an der Tuberositas gluteae als Zeichen einer verstärkten Beanspruchung des ansetzenden Muskels.

a: Trochanter major, $\rightarrow$ : Exostose.

Abb. 34 d: Grab 135 A; 9 bis 11 Jahre. Geschlecht unbestimmbar. Dorsalansicht des linken Femurs, proximales Drittel. Caudal des Collums befindet sich eine knotige Exostose an der Tuberositas glutea.

a: Sulcus m. vastus lateralis, b: Exostose an der Tuberositas glutea.

Abb. 34 e: Grab 318; 7,5 bis 10 Jahre. Geschlecht wahrscheinlich männlich. Medialansicht des rechten Femurs, proximales Drittel. Im Bereich des Trochanter minor befindet sich als Zeichen einer ausgeheilten Fraktur eine exostotische Neubildung $(\rightarrow)$.

*: ausgeheilte Frakturspalte.

Abb. 34 f: Grab 318; 7,5 bis 10 Jahre. Geschlecht wahrscheinlich männlich. Dorsalansicht auf Caput und Collum des rechten Femurs. Im Bereich des Trochanter minor zeigt sich bei Zustand nach Fraktur die Verwachsung des ehemals abgesprengten Knochenfragments an nicht originärer Stelle mit exostotischer Neubildung.

$\rightarrow$ : Trochanter minor. 
Tafel 34
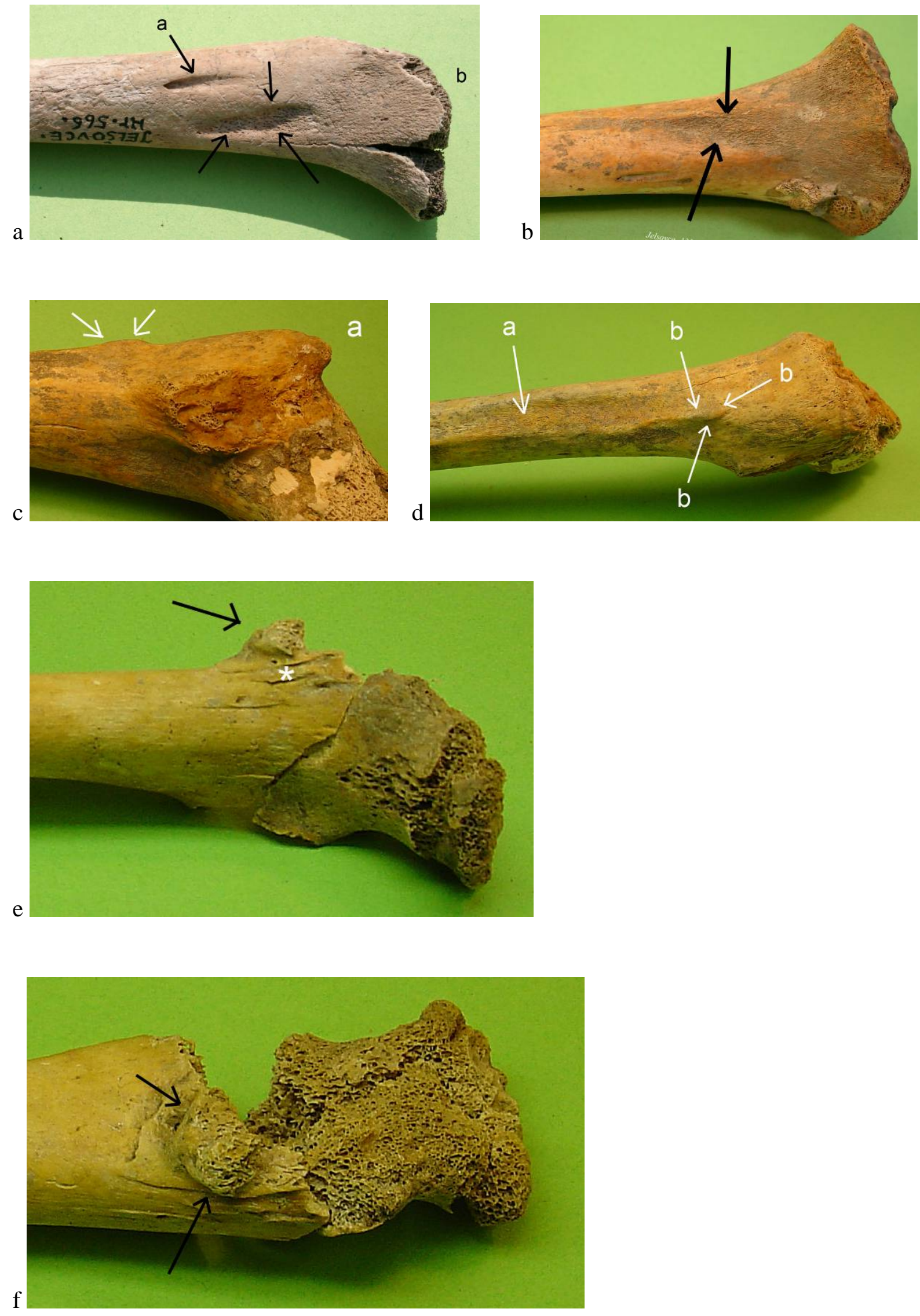
Abb. 35 a: Grab 134 B; 8 bis 11 Jahre. Geschlecht unbestimmbar. Rasterelektronenmikroskopische Aufnahme der rechten Tibia, Facies medialis im mittleren Bereich Diaphyse. Die ovalen, längsorientierten Foramina sind intravitalen Ursprungs. V.a. Reste einer gut integrierten postmortal erodierten Auflagerung.

Vergrößerung 11fach.

Abb. 35 b: Grab 134 B; 8 bis 11 Jahre. Geschlecht unbestimmbar. Betrachtung eines Knochendünnschliffs $(50 \mu \mathrm{m})$ aus der rechten Tibia im polarisierten Durchlicht unter Benutzung eines Hilfsobjekts Rot 1. Ordnung (Quarz) als Kompensator. Vergrößerung 16fach.

Die oberste Knochenschicht ist postmortal abgespalten. Der Sprung verläuft entlang der Kollagenfasern um ein Osteon herum.

Abb. 35 c: Grab 134 B; 8 bis 11 Jahre. Geschlecht unbestimmbar. Betrachtung eines Knochendünnschliffs $(50 \mu \mathrm{m})$ aus der rechten Tibia im polarisierten Durchlicht unter Benutzung eines Hilfsobjekts Rot 1. Ordnung (Quarz) als Kompensator. Detail aus Abb. 35 b. Vergrößerung 100fach.

Der postmortal Sprung läuft entlang der Kollagenfasern um das Osteon herum. +: versinterte Spalte, a: Haverskanal.

Abb. 35 d: Grab 134 B; 8 bis 11 Jahre. Geschlecht unbestimmbar. Betrachtung eines Knochendünnschliffs $(70 \mu \mathrm{m})$ aus der rechten Tibia im polarisierten Durchlicht unter Benutzung eines Hilfsobjekts Rot 1. Ordnung (Quarz) als Kompensator. Vergrößerung 100fach.

Die Oberfläche ist postmortal erodiert, darunter befinden sich intravital entstandene Gefäßspalten.

Abb. 35 e: Grab 134 B; 8 bis 11 Jahre. Geschlecht unbestimmbar. Betrachtung eines Knochendünnschliffs $(50 \mu \mathrm{m})$ aus der rechten Tibia im polarisierten Durchlicht unter Benutzung eines Hilfsobjekts Rot 1. Ordnung (Quarz) als Kompensator. Vergrößerung 100fach.

Postmortal erodierte Oberfläche, darunter befinden sich intravital entstandene Gefäßspalten. 
Tafel 35
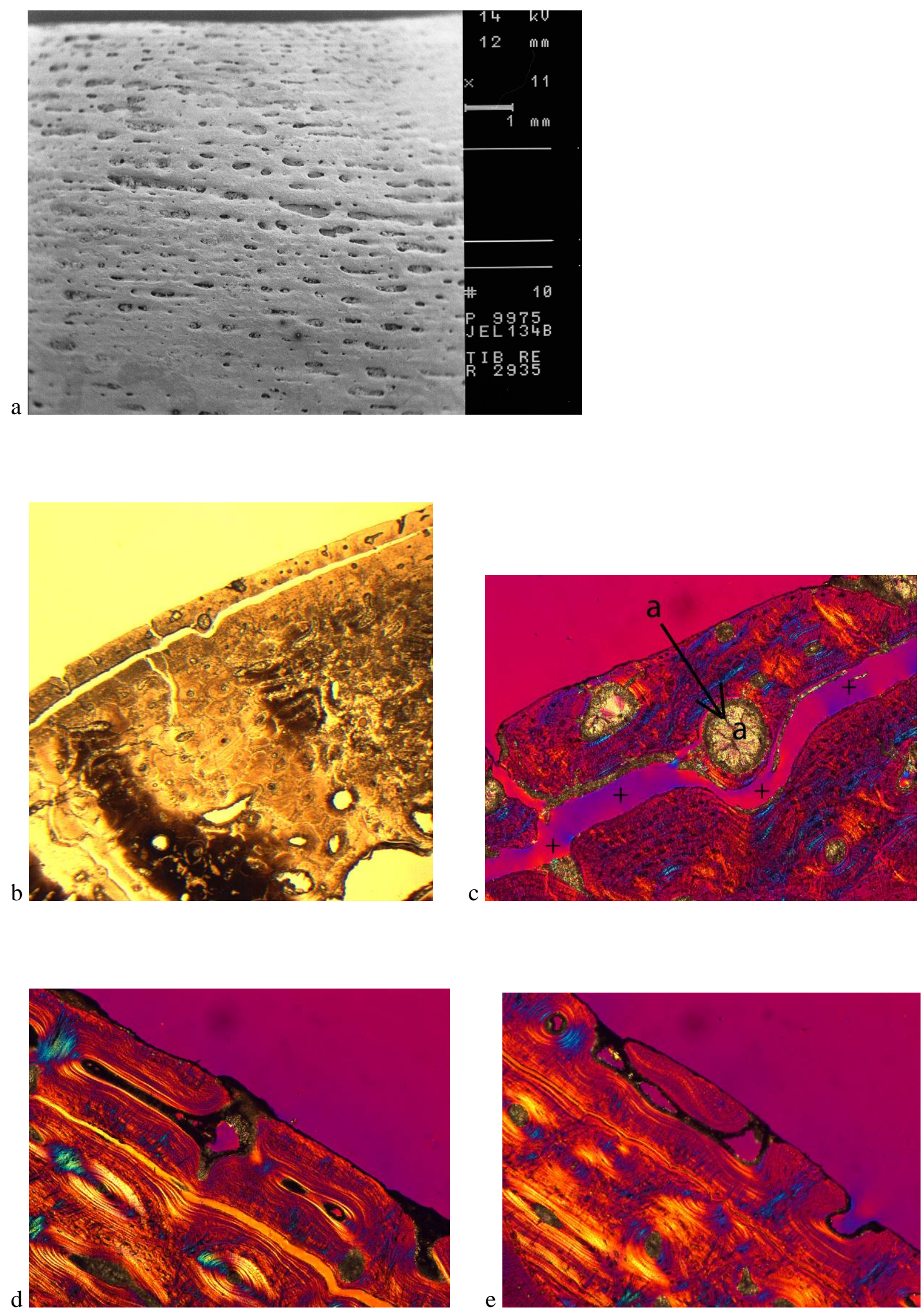
Abb. 36 a: Grab 626; 5 bis 8 Jahre. Mittlerer Diaphysenabschnitt der linken Fibula. Auf dem Margo anterior sind mehrere plaqueartigen Auflagerungen $(\rightarrow)$ zu erkennen.

Abb. 36 b: Grab 626; 5 bis 8 Jahre. Geschlecht unbestimmbar. Betrachtung eines Knochendünnschliffs $(50 \mu \mathrm{m})$ aus der linken Fibula im polarisierten Durchlicht unter Benutzung eines Hilfsobjekts Rot 1. Ordnung (Quarz) als Kompensator. Vergrößerung 16fach.

Die subperiostal gelegene, plaqueartige Auflagerung (schwarzer Pfeil) ist im zentralen Bereich getroffen. Reste dieser Auflagerung setzen sich in Richtung des Margo interosseus fort (weißer Pfeil).

a: Markröhre.

Abb. 36 c: Grab 626; 5 bis 8 Jahre. Geschlecht unbestimmbar. Betrachtung eines Knochendünnschliffs $(50 \mu \mathrm{m})$ aus der linken Fibula im polarisierten Durchlicht unter Benutzung eines Hilfsobjekts Rot 1. Ordnung (Quarz) als Kompensator. Detail aus Abb. 36 b. Vergrößerung 25fach.

Die plaqueartige Auflagerung liegt der ehemaligen Oberfläche auf (+). Es handelt sich um die Reste einer hämorrhagisch bedingten Auflagerung.

+ : äußere Generallamelle.

Abb. 36 d: Grab 626; 5 bis 8 Jahre. Geschlecht unbestimmbar. Betrachtung eines Knochendünnschliffs $(70 \mu \mathrm{m})$ aus der linken Fibula im polarisierten Durchlicht unter Benutzung eines Hilfsobjekts Rot 1. Ordnung (Quarz) als Kompensator. Vergrößerung 16fach.

Die Auflagerung ist im dickeren Schliff $(70 \mu \mathrm{g})$ nur im Randbereich getroffen und nur als schmaler Saum erhalten $(\rightarrow)$. Sie ist zum Teil postmortal abgemürbt.

Abb. 36 e: Grab 626; 5 bis 8 Jahre. Geschlecht unbestimmbar. Betrachtung eines Knochendünnschliffs $(50 \mu \mathrm{m})$ aus der linken Fibula im polarisierten Durchlicht unter Benutzung eines Hilfsobjekts Rot 1. Ordnung (Quarz) als Kompensator. Detail aus Abb. 36 c. Vergrößerung 200fach.

Die Auflagerung ist bereits gut organisiert. Der gut differentierte Faserknochen wurde zum Todeszeitpunkt gerade in Lamellenknochen umgebaut. Erste Haverssysteme (a) durchziehen den Knochen.

a: Haverssysteme, +: ehemalige Oberfläche, äußere Generallamelle. 
Tafel 36
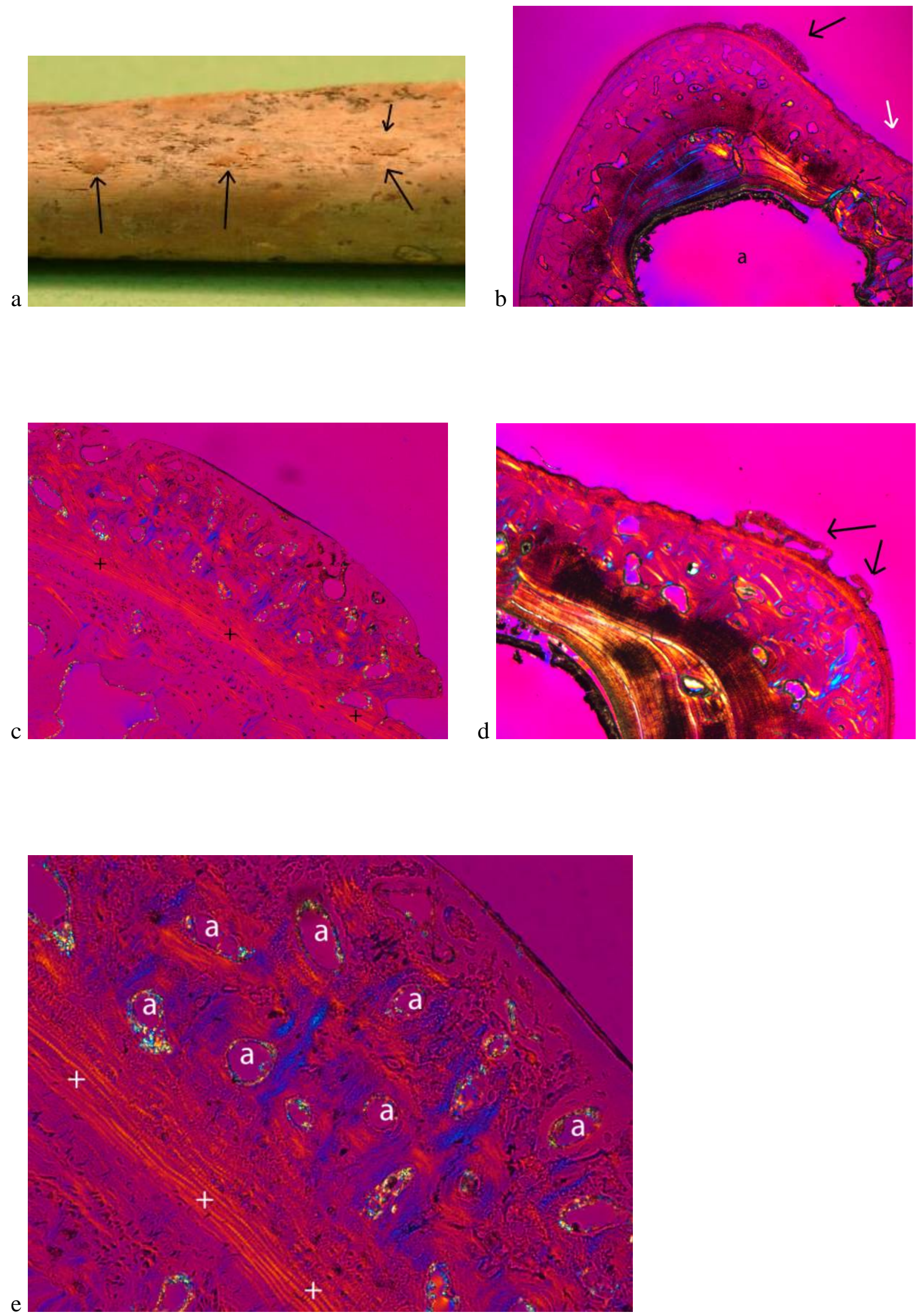
Abb. 37 a: Grab 626; 5 bis 8 Jahre. Geschlecht unbestimmbar. Betrachtung eines Knochendünnschliffs $(50 \mu \mathrm{m})$ aus der linken Fibula im polarisierten Durchlicht unter Benutzung eines Hilfsobjekts Rot 1. Ordnung (Quarz) als Kompensator. Vergrößerung 16fach.

Auch an der gegenüberliegenden Seite der deutlich erkennbaren plaqueartige Auflagerung (Facies dorsalis) sind Reste einer subperiostal gelegenen Knochenneubildung (a) zu erkennen. Die darunterliegende Compacta ist von zahlreichen Resorptionshöhlen durchzogen.

a: Reste einer Auflagerung, b: Resorptionshöhlen, c: Markröhre

Abb. 37 b: Grab 626; 5 bis 8 Jahre. Geschlecht unbestimmbar. Betrachtung eines Knochendünnschliffs $(50 \mu \mathrm{m})$ aus der linken Fibula im polarisierten Durchlicht unter Benutzung eines Hilfsobjekts Rot 1. Ordnung (Quarz) als Kompensator. Vergrößerung 200fach.

Detail einer Resorptionshöhle, die sich unterhalb der Auflagerung in der Compacta befindet. Die Ränder sind unregelmäßig begrenzt. Verschiedenen Howshipsche Lakunen sind erkennbar.

a: Resorptionshöhle.

Abb. 37 c: Grab 626; 5 bis 8 Jahre. Geschlecht unbestimmbar. Betrachtung eines Knochendünnschliffs $(50 \mu \mathrm{m})$ aus der linken Fibula im polarisierten Durchlicht unter Benutzung eines Hilfsobjekts Rot 1. Ordnung (Quarz) als Kompensator. Vergrößerung 200fach.

Detail einer Resorptionshöhle in der Compacta unterhalb der Auflagerung. Es zeigen sich zahlreiche Howshipsche Lakunen $(\rightarrow)$.

a: Resorptionshöhle, $\rightarrow$ : Howshipsche Lakunen. 
Tafel 37
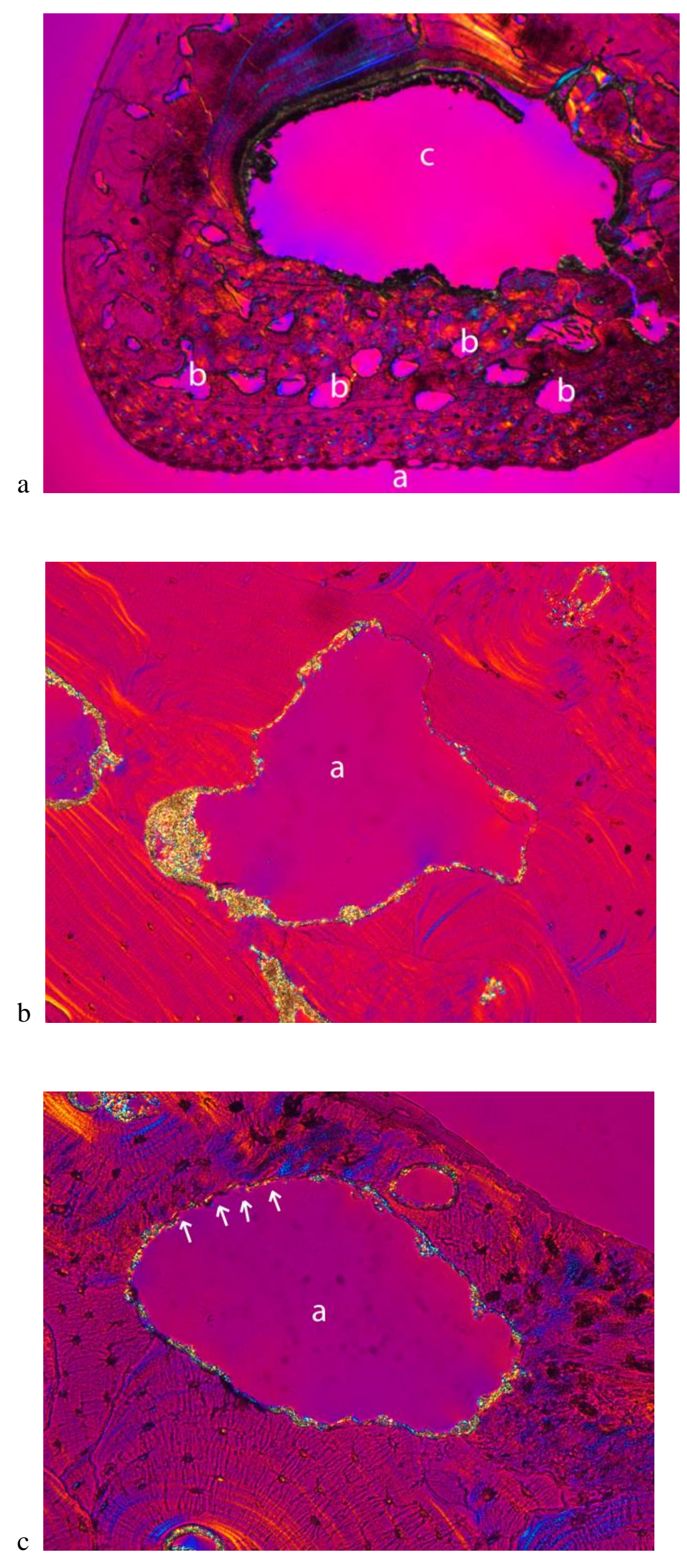
Tafel 38

Abb. 38 a: Grab 299; 9 bis 10,5 Jahre. Geschlecht unbestimmbar. Mittlerer Diaphysenabschnitt der rechten Fibula. Die Oberfläche der Facies medialis ist streifig porös $(\rightarrow)$.

Abb. 38 b: Grab 299; 9 bis 10,5 Jahre. Geschlecht unbestimmbar. Mittlerer Diaphysenabschnitt der rechten Fibula. Lupenmikroskopische Aufnahme der Facies medialis. Auf der streifigen Oberfläche befinden sich konfluierende Knochenplatten. Im Randbereich ist die Oberfläche postmortal zerstört.

Abb. 38 c: Grab 299; 9 bis 10,5 Jahre. Geschlecht unbestimmbar. Mittlerer Diaphysenabschnitt der rechten Fibula. Lupenmikroskopische Aufnahme der Facies medialis. Die Oberfläche ist stark längsgestreift porös.

Abb. 38 d: Grab 299; 9 bis 10,5 Jahre. Geschlecht unbestimmbar. Betrachtung eines Knochendünnschliffs $(50 \mu \mathrm{m})$ aus der rechten Fibula im polarisierten Durchlicht unter Benutzung eines Hilfsobjekts Rot 1. Ordnung (Quarz) als Kompensator. Detail aus Abb. 38 e. Vergrößerung 100fach.

Auf der äußeren Generallamelle $(+)$ sind die Reste einer ehemals subperiostal gelegenen Auflagerung zu erkennen.

+: ehemalige Oberfläche.

Abb. 38 e: Grab 299; 9 bis 10,5 Jahre. Geschlecht unbestimmbar. Betrachtung eines Knochendünnschliffs $(50 \mu \mathrm{m})$ aus der rechten Fibula im polarisierten Durchlicht unter Benutzung eines Hilfsobjekts Rot 1. Ordnung (Quarz) als Kompensator. Detail aus Abb. 38 e. Vergrößerung 25fach.

Auf großen Teilen der Oberfläche sind die Reste einer subperiostal gelegenen, hämorrhagisch bedingten Auflagerung $(\rightarrow)$ zu erkennen. 
Tafel 38
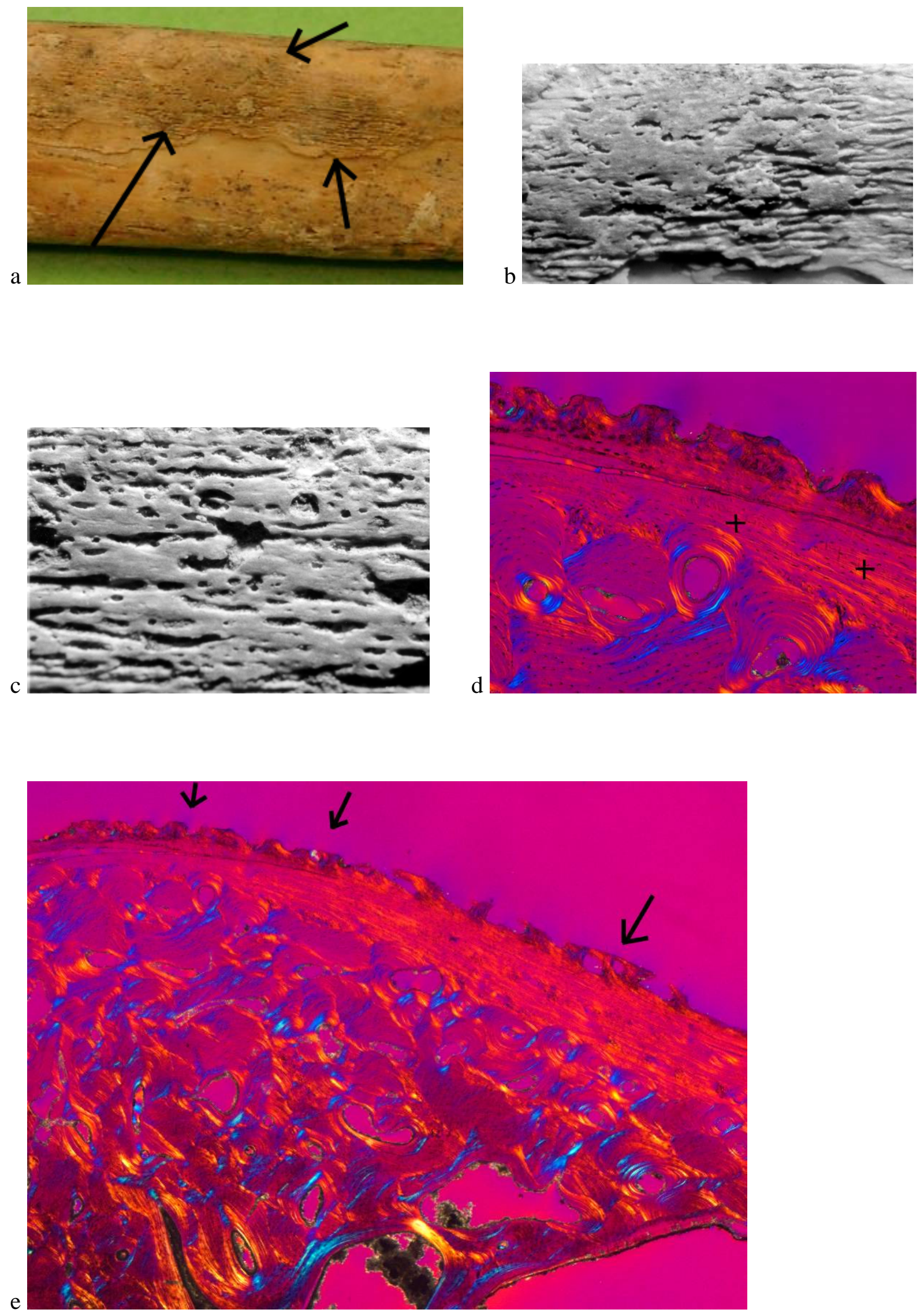
Abb. 39 a: Grab 41; 2,5 bis 4 Jahre. Geschlecht unbestimmbar. Röntgenaufnahme des proximalen Abschnitts der linken Tibia. Strahlengang ap, 50 kV, Belichtungszeit 5 Min. Im metaphysären Schaft kommen verschiedene Harris-Linien zur Darstellung.

Abb. 39 b: Grab 300 A; 2 bis 3 Jahre. Geschlecht unbestimmbar. Röntgenaufnahme des distalen Abschnitts der linken Fibula. Strahlengang ap, 50 kV, Belichtungszeit 4,5 Min.

Verschiedene Harris-Linien sind erkennbar (-).

Abb. 39 c: Grab 300 A; 2 bis 3 Jahre. Geschlecht unbestimmbar. Röntgenaufnahme des distalen Abschnitts von Fibula und Tibia der rechten Seite. Strahlengang ap, $50 \mathrm{kV}$, Belichtungszeit 4,5 Min.

An beiden Knochen kommen verschiedene Harris-Linien (-) zur Darstellung.

Abb. 39 d: Grab 126; 9 bis 11 Jahre. Geschlecht weiblich. Ventralansicht auf Caput und Collum des rechten Femurs. Der Bereich des ventromedialen Collumfläche ist verstärkt porös $(\rightarrow)$.

Abb. 39 e: Grab 113; 9,5 bis 12 Jahre. Geschlecht unbestimmbar. Medialansicht des rechten Femur. Im Bereich des ventromedialen Collumfläche befindet sich ein poröses Areal $(\rightarrow)$. 
Tafel 39
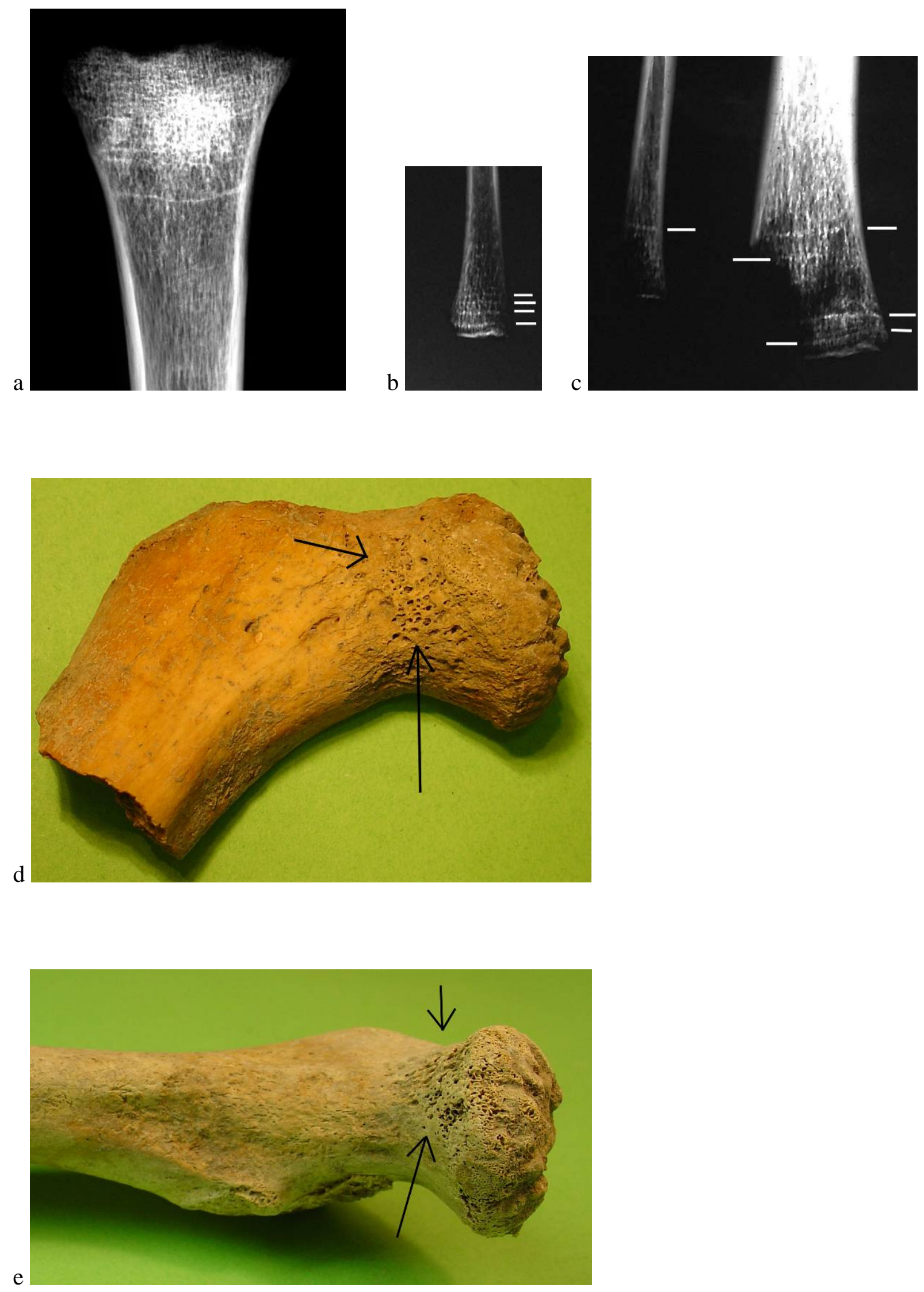
Abb. 40 a: Grab 616 B; 9 bis 11 Jahre. Geschlecht weiblich. Medialansicht der linken Tibia. Der Knochen ist besonders im proximalen Abschnitt verdickt und ist nach dorsal verbogen.

Abb. 40 b: Grab 616 B; 9 bis 11 Jahre. Geschlecht weiblich. Linke Tibia, Detailaufnahme der proximalen Epiphyse (im Bild links) und angrenzender Diaphyse (im Bild rechts). Von der Epiphyse aufgehend zeigt sich ein atypischer Verknöcherungssporn in die Diaphyse reichend (a). Die proximale Metaphysenplatte der Diaphyse (b) ist nicht wie zu erwarten glatt begrenzt. Sie weist einen in die Tiefe reichenden Spongiosaverlust so wie atypische Spongiosa auf.

a: epiphysärer Verknöcherungssporn, b: proximale Metaphysenplatte der Diaphyse, c: atypische Spongiosastrukturen, *: Tuberositas tibiae.

Abb. 40 c: Grab 616 B; 9 bis 11 Jahre. Geschlecht weiblich. Blick auf die proximale Metaphysenplatte der Diaphyse der linken Tibia. Die Metaphysenplatte ist nicht - wie zu erwarten wäre - geschlossen. Neben atypischer Spongiosa ist ein in die Tiefe reichender Spongiosaverlust erkennbar.

Abb. 40 d: Grab 616 B; 9 bis 11 Jahre. Geschlecht weiblich. Linke Tibia, Detailaufnahme von Epiphyse und angrenzender Diaphyse in ehemaliger Lage. Von der Epiphyse aufgehend zeigt sich ein atypischer in die Diaphyse reichender Verknöcherungssporn. Die Verbindung wurde zum Teil postmortal zerstört. 
Tafel 40

a
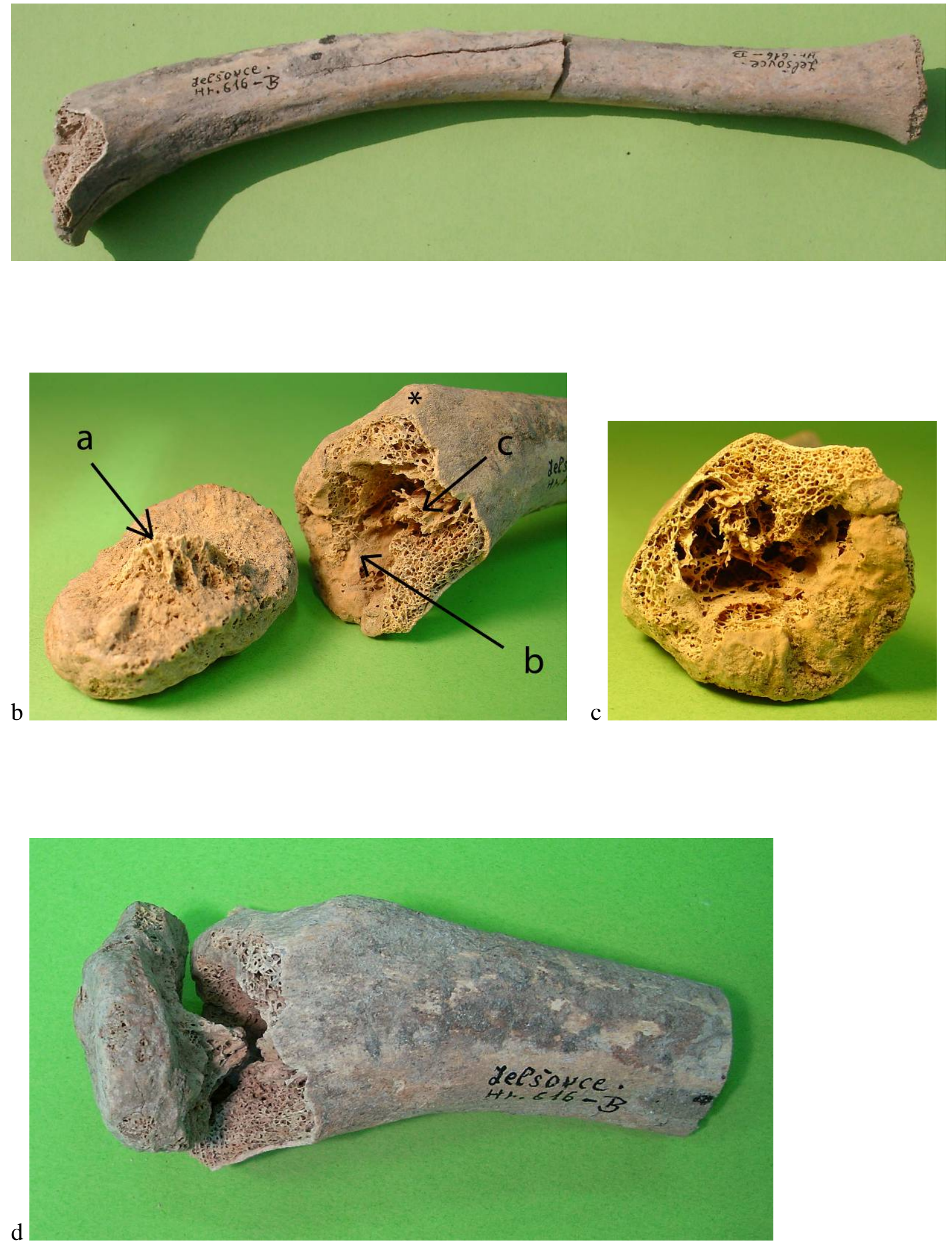
Abb. 41 a: Grab 616 B; 9 bis 11 Jahre. Geschlecht weiblich. Endoskopische Aufnahme der Markröhre im distalen Diaphyseabschnitt der linken Tibia. Über der normal konfigurierten Spongiosa befindet sich eine Lage sehr feiner, atypischer fadenförmiger Spongiosa.

Abb. 41 b: Grab 616 B; 9 bis 11 Jahre. Geschlecht weiblich. Endoskopische Aufnahme aus dem mittleren Diaphyseabschnitt der linken Tibia. Die Markröhre ist vollständig mit feiner atypischer Spongiosa ausgefüllt, an einer Stelle, an der keine Spongiosa zu erwarten ist.

Abb. 41 c: Grab 616 B; 9 bis 11 Jahre. Geschlecht weiblich. Betrachtung eines Knochendünnschliffs $(50 \mu \mathrm{m})$ aus der linken Tibia im distalen Drittel. Dünnschliffübersicht. Vergrößerung 3fach.

Der untere Teil der Markröhre ist vollständig mit atypisch feiner Spongiosa ausgefüllt (*), die von dystrophischen Bälkchen aufgebaut wird. Im Bereich der makroskopisch erkennbaren Vorwölbung der medialen Oberfläche zeigt sich eine Ausdünnung der Compacta $(\rightarrow)$.

Abb. 41 d: Grab 616 B; 9 bis 11 Jahre. Geschlecht weiblich. Betrachtung eines Knochendünnschliffs $(50 \mu \mathrm{m})$ aus der linken Tibia im distalen Drittel im polarisierten Durchlicht unter Benutzung eines Hilfsobjekts Rot 1. Ordnung (Quarz) als Kompensator. Vergrößerung 16 fach.

Neben normaler Spongiosa (im Bild oben) nahe der Compacta gelegen, ist in der Markröhre (unten) atypisch konfigurierte, feinere Spongiosa zu erkennen.

Abb. 41 e: Grab 616 B; 9 bis 11 Jahre. Geschlecht weiblich. Betrachtung eines Knochendünnschliffs $(50 \mu \mathrm{m})$ aus der linken Tibia im distalen Drittel im polarisierten Durchlicht unter Benutzung eines Hilfsobjekts Rot 1. Ordnung (Quarz) als Kompensator. Vergrößerung 16fach.

Im Markraum befinden sich unregelmäßig angeordnete dystrophische Spongiosabälkchen. 
Tafel 41
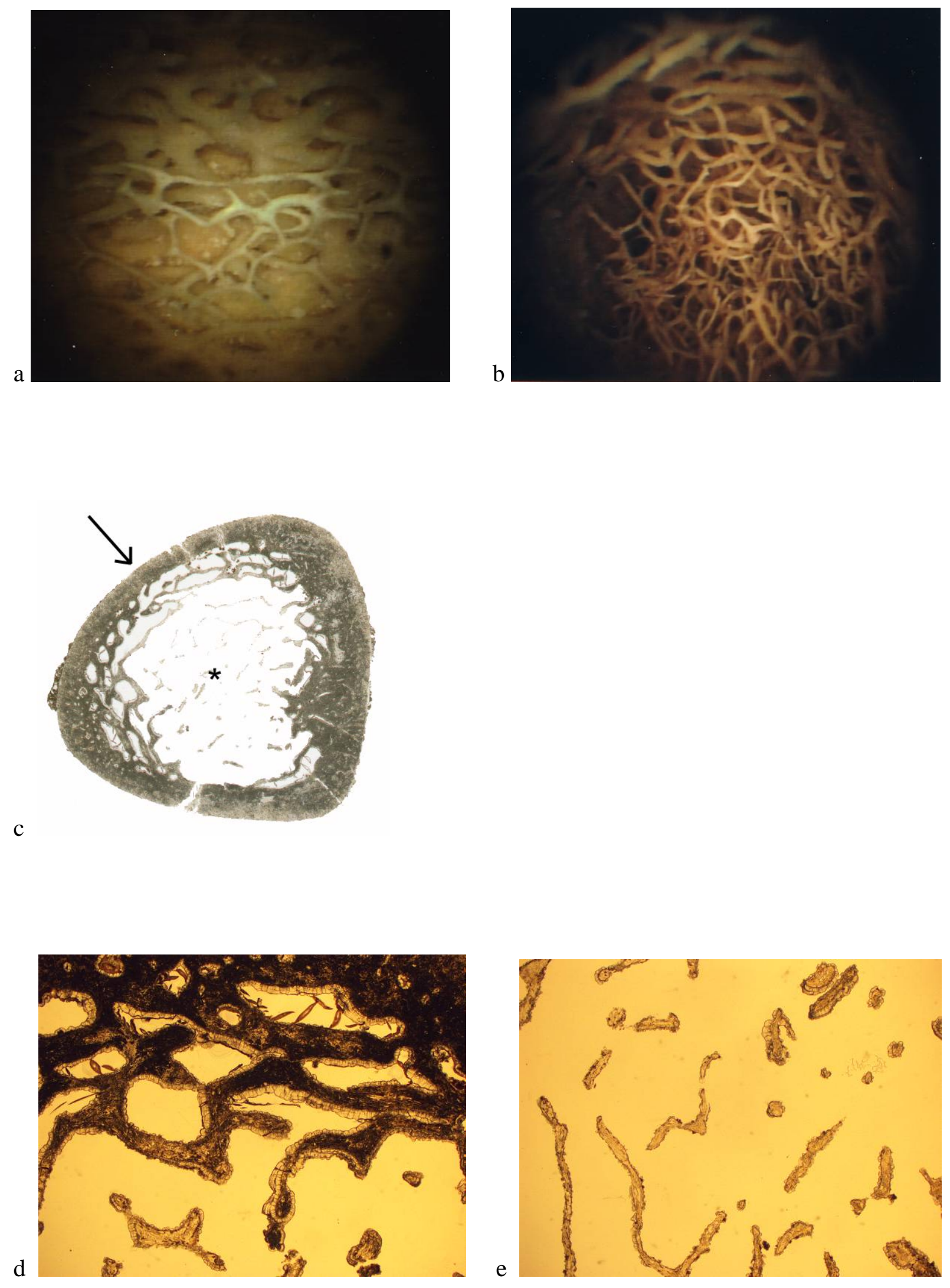
Abb. 42 a: Grab 300 A; 2 bis 3 Jahre. Geschlecht unbestimmbar. Ventralansicht der linken Tibia und Fibula. Beide Knochen sind makroskopisch normal konfiguriert.

Abb. 42 b: Grab 300 A; 2 bis 3 Jahre. Geschlecht unbestimmbar. Ventralansicht der rechten Tibia. Der Knochen ist verplumpt, hat abgerundete Kanten und ist nach medial gebogen.

Abb. 42 c: Grab 300 A; 2 bis 3 Jahre. Geschlecht unbestimmbar. Ventralansicht der rechten Fibula. Der Knochen ist verplumpt, hat abgerundete Kanten und ist nach medial gebogen.

Abb. 42 d: Grab 300 A; 2 bis 3 Jahre. Geschlecht unbestimmbar. Betrachtung eines Knochendünnschliffs $(70 \mu \mathrm{m})$ aus der linken Tibia in Diaphysenmitte. Dünnschliffübersicht. Vergrößerung 4fach.

Abb. 42 e: Grab 300 A; 2 bis 3 Jahre. Geschlecht unbestimmbar. Betrachtung eines Knochendünnschliffs $(70 \mu \mathrm{m})$ aus der rechten Tibia in Diaphysenmitte. Dünnschliffübersicht. Vergrößerung 4fach.

Abb. 42 f: Grab 300 A; 2 bis 3 Jahre. Geschlecht unbestimmbar. Betrachtung eines Knochendünnschliffs $(70 \mu \mathrm{m})$ aus der linken Fibula in Diaphysenmitte. Dünnschliffübersicht Vergrößerung 4fach.

Abb. 42 g: Grab 300 A; 2 bis 3 Jahre. Geschlecht unbestimmbar. Betrachtung eines Knochendünnschliffs $(70 \mu \mathrm{m})$ aus der rechten Fibula in Diaphysenmitte. Dünnschliffübersicht Vergrößerung 4fach. 
Tafel 42
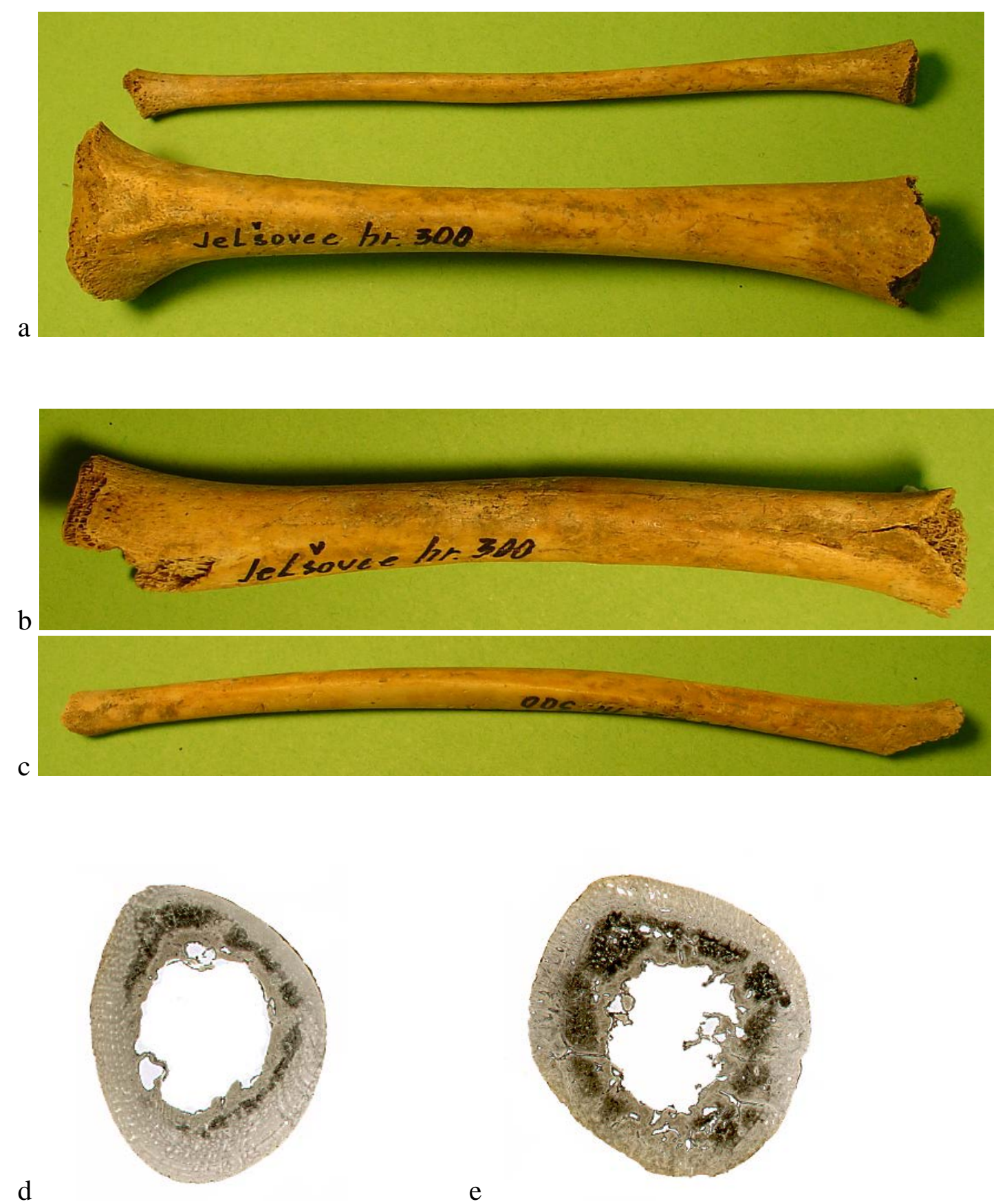

e
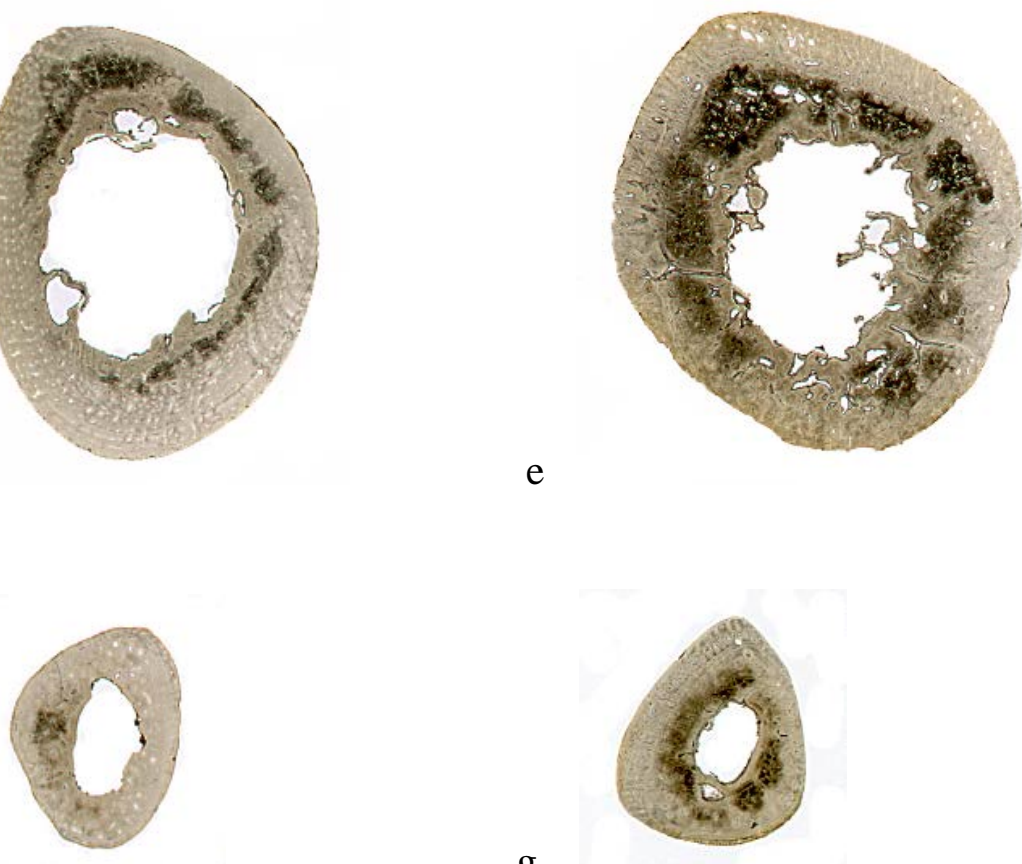

g 
Abb. 43 a, b und c: Grab 300 A; 2 bis 3 Jahre. Geschlecht unbestimmbar. Betrachtung eines Knochendünnschliffs $(50 \mu \mathrm{m})$ aus der linken Tibia im einfachen Durchlicht (Abb. $43 \mathrm{a}$ ), im polarisierten Durchlicht (Abb. 43 b) bzw. im polarisierten Durchlicht unter Benutzung eines Hilfsobjekts Rot 1. Ordnung (Quarz) als Kompensator (Abb. 43 c). Vergrößerung 16fach.

Auf der Oberfläche zeigt sich eine massive Knochenneubildung. a: Markröhre, b: äußere Oberfläche, $\leftrightarrow$ : Neubildung.

Abb. 43 d: Grab 300 A; 2 bis 3 Jahre. Geschlecht unbestimmbar. Betrachtung eines Knochendünnschliffs $(70 \mu \mathrm{m})$ aus der linken Tibia im polarisierten Durchlicht unter Benutzung eines Hilfsobjekts Rot 1. Ordnung (Quarz) als Kompensator. Vergrößerung 16fach.

Die Neubildung ist im Randbereich getroffen. Ein versinterter Gefäßkanal (b) zieht von der Markröhre in die Knochenneubildung (a).

a: Neubildung, b: Gefäßkanal.

Abb. 43 e: Grab 300 A; 2 bis 3 Jahre. Geschlecht unbestimmbar. Betrachtung eines Knochendünnschliffs $(50 \mu \mathrm{m})$ aus der linken Tibia im polarisierten Durchlicht unter Benutzung eines Hilfsobjekts Rot 1. Ordnung (Quarz) als Kompensator. Detail aus Abb. 43 c. Vergrößerung 100fach.

Die Detailaufnahme der Neubildung einschließlich der äußeren Oberfläche zeigt senkrecht zur Oberfläche orientierte Kollagenstrukturen.

Abb. 43 f: Grab 300 A; 2 bis 3 Jahre. Geschlecht unbestimmbar. Betrachtung eines Knochendünnschliffs $(50 \mu \mathrm{m})$ aus der linken Tibia im polarisierten Durchlicht unter Benutzung eines Hilfsobjekts Rot 1. Ordnung (Quarz) als Kompensator. Detail aus Abb. 43 c. Vergrößerung 100fach.

Die Detailaufnahme zeigt den Übergang der Neubildung zur ehemaligen Oberfläche (a). a: ehemalige Oberfläche, b: Neubildung. 
Tafel 43
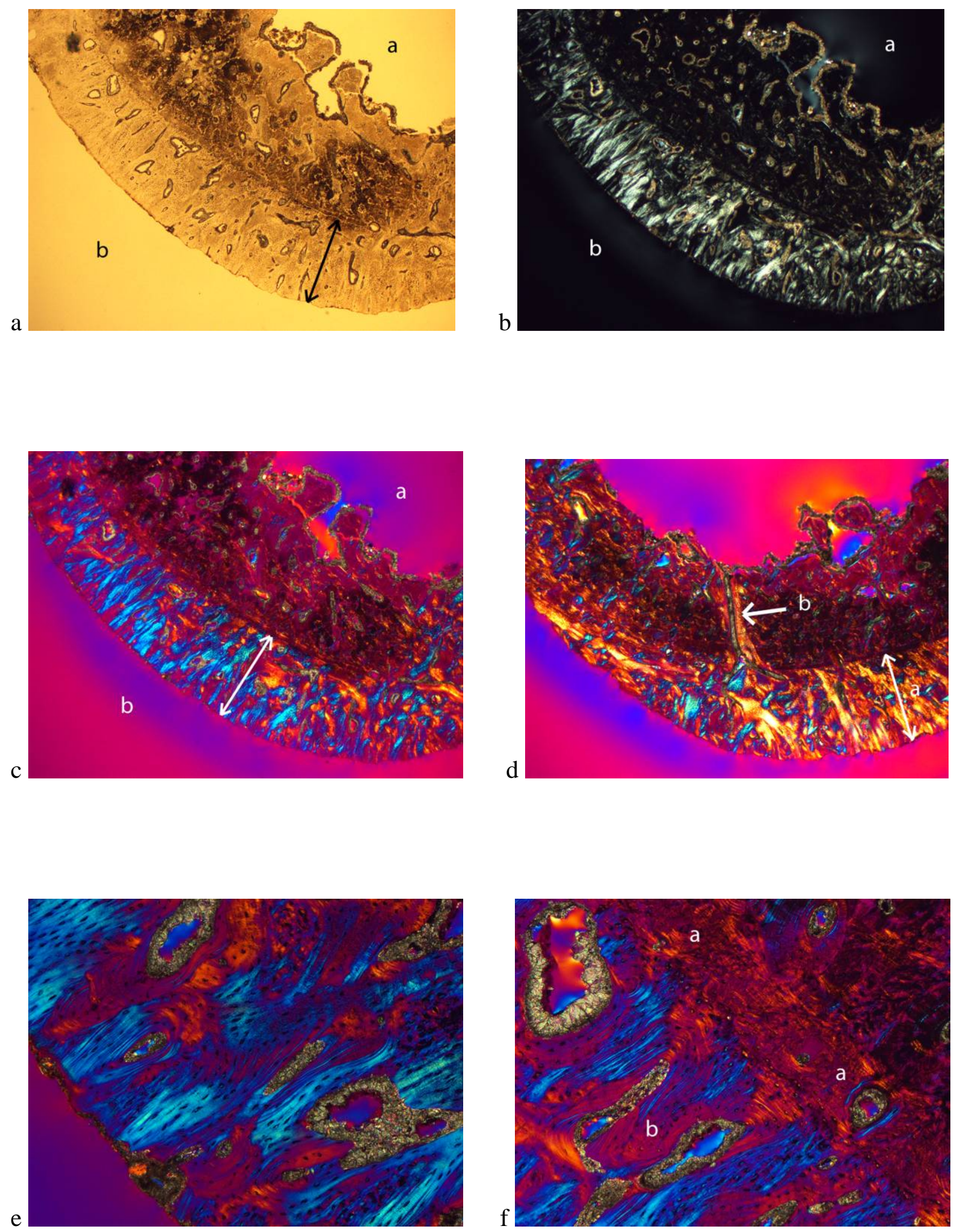
Abb. 44 a: Grab 300 A; 2 bis 3 Jahre. Geschlecht unbestimmbar. Betrachtung eines Knochendünnschliffs $(50 \mu \mathrm{m})$ aus der linken Fibula im polarisierten Durchlicht unter Benutzung eines Hilfsobjekts Rot 1. Ordnung (Quarz) als Kompensator. Vergrößerung 25fach.

Der äußeren Generallamelle aufgelagert befindet sich eine osteonenkanalreiche Knochenneubildung. a: Markröhre, *: ehemalige Oberfläche, äußere Generallamelle, $\rightarrow$ : Neubildung.

Abb. 44 b: Grab 300 A; 2 bis 3 Jahre. Geschlecht unbestimmbar. Betrachtung eines Knochendünnschliffs $(50 \mu \mathrm{m})$ aus der linken Fibula im polarisierten Durchlicht unter Benutzung eines Hilfsobjekts Rot 1. Ordnung (Quarz) als Kompensator. Detail aus Abb. 44 a. Vergrößerung 100fach.

Die osteonenkanalreiche Neubildung ist der ehemaligen Oberfläche aufgelagert. a: ehemalige Oberfläche, äußere Generallamelle, b: äußere Oberfläche.

Abb. 44 c: Grab 15; 10 bis 16 Monate. Geschlecht unbestimmbar. Betrachtung eines Knochendünnschliffs $(50 \mu \mathrm{m})$ aus der linken Tibia im polarisierten Durchlicht unter Benutzung eines Hilfsobjekts Rot 1. Ordnung (Quarz) als Kompensator. Vergrößerung 100fach.

Ein zur Markröhre hin gelegenes Haverssystem ist eröffnet (*). Zu beiden Seiten befindet sich ein Band von Howshipschen Lakunen als Zeichen einer von der Markröhre ausgehenden Resorption des Knochens.

*: eröffnetes Haverssystem, $\rightarrow$ : Howshipsche Lakunen.

Abb. 44 d: Grab 15; 10 bis 16 Monate. Betrachtung eines Knochendünnschliffs $(50 \mu m)$ aus der linken Tibia im polarisierten Durchlicht unter Benutzung eines Hilfsobjekts Rot 1. Ordnung (Quarz) als Kompensator. Detail aus Abb. 44 c. Vergrößerung 250fach.

$\mathrm{Zu}$ beiden Seiten des zur Markröhre hin gelegenen, eröffneten Haverssystems $\left(^{*}\right)$ befindet sich ein Band von Howshipschen Lakunen als Zeichen der von der Markröhre ausgehenden Resorption des Knochens. Deutlich erkennbar ist die für Howshipsche Lakunen im Knochendünnschliff typische Doppelkonturierung. Im Gegensatz zur normalen Resorption sind die Ränder sehr unregelmäßig ausgeprägt (vgl. Abb. 46 d).

*: eröffnetes Haverssystem, $\rightarrow$ : Howshipsche Lakunen. 
Tafel 44
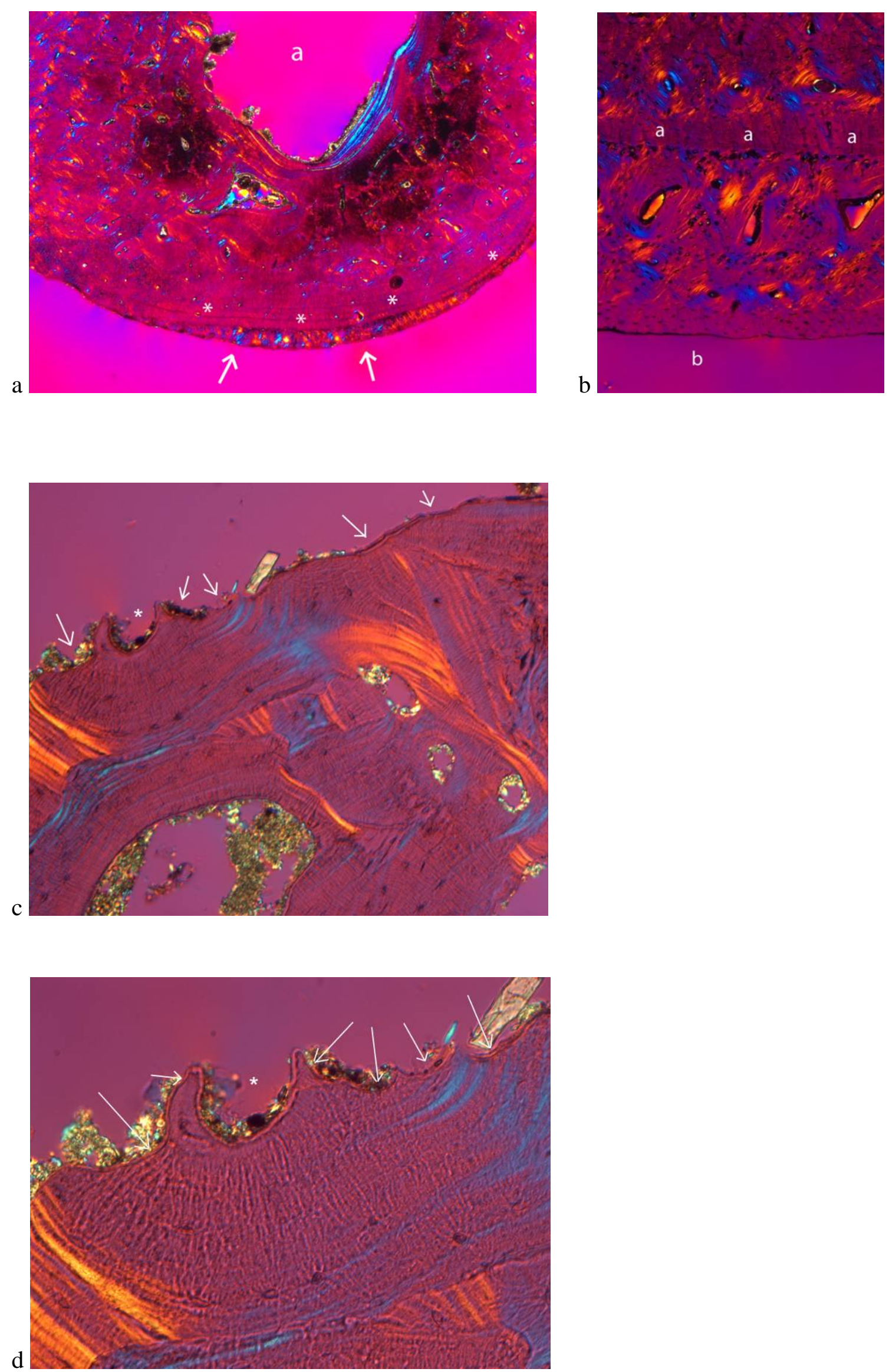
Abb. 45 a: Grab 15; 10 bis 16 Monate. Geschlecht unbestimmbar. Betrachtung eines Knochendünnschliffs $(70 \mu \mathrm{m})$ aus der linken Tibia in Diaphysenmitte. Dünnschliffübersicht. Vergrößerung 4fach.

Abb. 45 b: Grab 15; 10 bis 16 Monate. Geschlecht unbestimmbar. Betrachtung eines Knochendünnschliffs $(70 \mu \mathrm{m})$ aus der rechten Femurs in Diaphysenmitte. Dünnschliffübersicht

Vergrößerung 4fach.

Abb. 45 c: Grab 15; 10 bis 16 Monate. Geschlecht unbestimmbar. Ventralansicht von Femur und Tibia der rechten Seite. Beide Knochen sind insgesamt sehr grazil gebaut.

Abb. 45 d: Grab 15; 10 bis 16 Monate. Geschlecht unbestimmbar. Ventralansicht von Femur und Tibia der linken Seite. Beide Knochen weisen einen sehr grazilen Knochenbau auf.

Abb. 45 e: Grab 15; 10 bis 16 Monate. Geschlecht unbestimmbar. Betrachtung eines Knochendünnschliffs $(50 \mu \mathrm{m})$ aus dem rechten Femurs im polarisierten Durchlicht unter Benutzung eines Hilfsobjekts Rot 1. Ordnung (Quarz) als Kompensator. Vergrößerung 25fach.

Äußere Generallamelle und Spongiosa sind deutlich schmaler ausgebildet als der Erwartung entspricht.

a: Compacta, b: Spongiosa, c: Markhöhle.

Abb. 45 f: Grab 15; 10 bis 16 Monate. Geschlecht unbestimmbar. Betrachtung eines Knochendünnschliffs $(70 \mu \mathrm{m})$ aus dem rechten Femurs im polarisierten Durchlicht unter Benutzung eines Hilfsobjekts Rot 1. Ordnung (Quarz) als Kompensator. Vergrößerung 25fach.

Die Compacta ist sehr schmal ausgebildet. In Richtung der Markröhre sind, in einem Bereich, in dem eigentlich noch Compacta sein sollte, zwei tangentiale Spongiosabälkchen (b) gelegen. a: Compacta, b: Spongiosa. 
Tafel 45

C

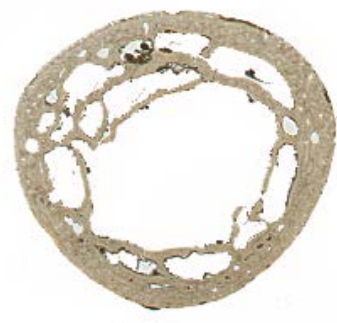

$\mathrm{a}$

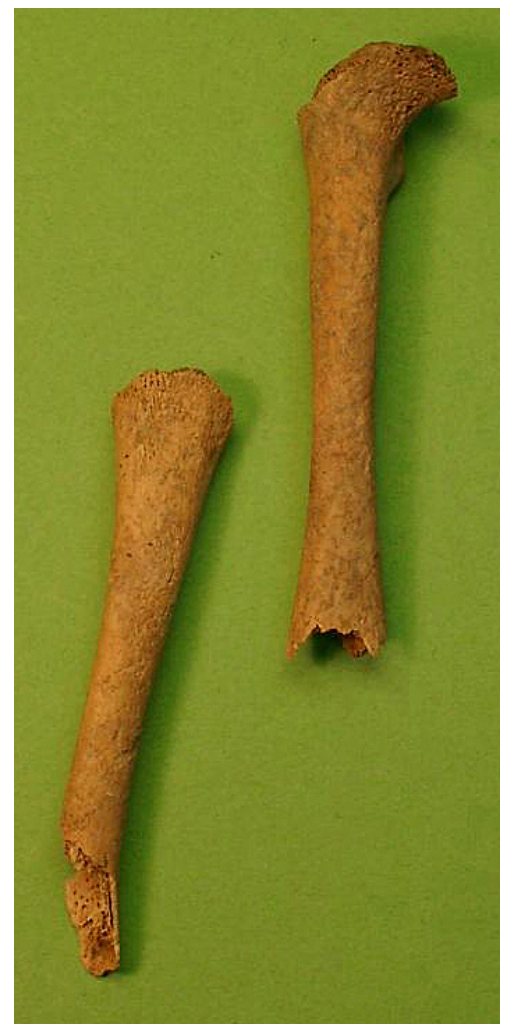

b

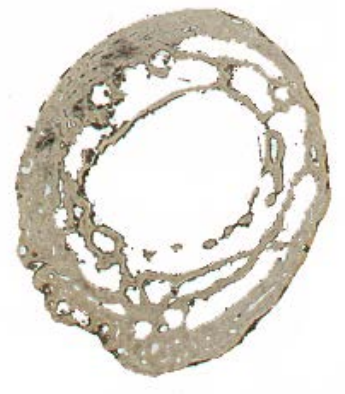

d

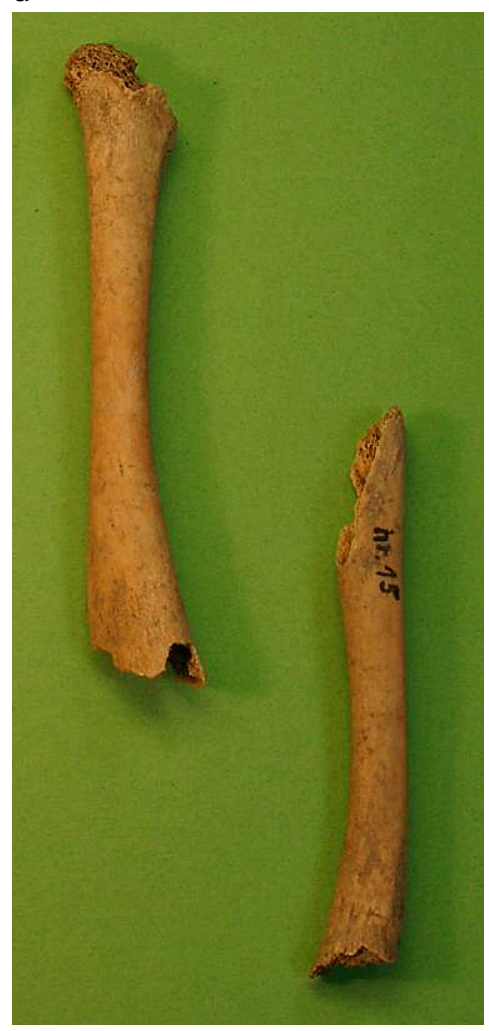

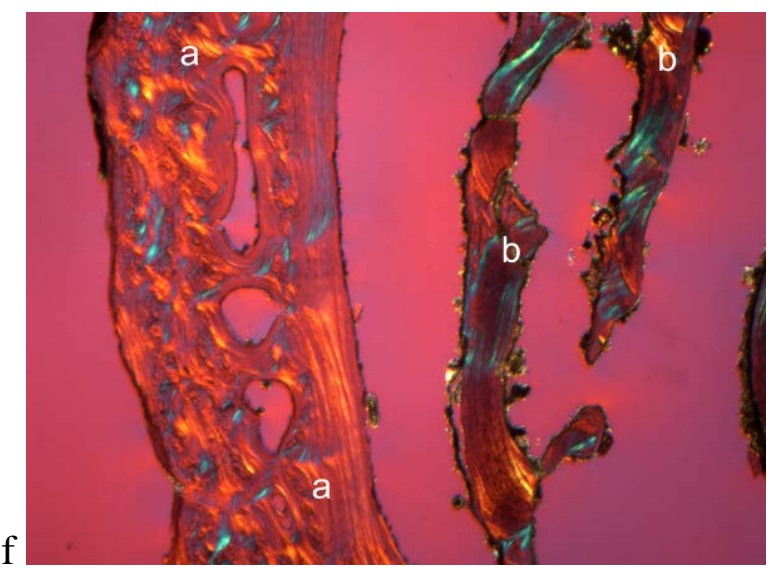


Abb. 46 a: Grab 15; 10 bis 16 Monate. Geschlecht unbestimmbar. Betrachtung eines Knochendünnschliffs $(50 \mu \mathrm{m})$ aus dem rechten Femur im polarisierten Durchlicht unter Benutzung eines Hilfsobjekts Rot 1. Ordnung (Quarz) als Kompensator. Vergrößerung 25fach.

Compacta (b) und Spongiosa (a) sind stark ausgedünnt. Es sind Zeichen von resorptiven Prozessen im Knochen nachweisbar.

*: Haverssystem mit Howshipschen Lagunen (Detail s. Abb. 46 c).

Abb. 46 b: Grab 15; 10 bis 16 Monate. Geschlecht unbestimmbar. Betrachtung eines Knochendünnschliffs $(70 \mu \mathrm{m})$ aus dem rechten Femur im polarisierten Durchlicht unter Benutzung eines Hilfsobjekts Rot 1. Ordnung (Quarz) als Kompensator. Vergrößerung 25fach.

Der Ausschnitt zeigt die Compacta im Bereich der Linea aspera (a). Der sich darstellende Faserknochen ist ein Zeichen dafür, dass das Bein aktiv bewegt wurde. Es fand eine Knochenum- und -neubildung durch den Muskelzug am Knochen statt.

a: Faserknochen im Bereich der Linea aspera, b: kleine Exostose an der äußeren Lamelle, c: Lamellenknochen, erhaltener Rest der ehemaligen Kompakta, *: Resorptionsspalte.

Abb. 46 c: Grab 15; 10 bis 16 Monate. Geschlecht unbestimmbar. Betrachtung eines Knochendünnschliffs $(50 \mu \mathrm{m})$ aus dem rechten Femur im polarisierten Durchlicht unter Benutzung eines Hilfsobjekts Rot 1. Ordnung (Quarz) als Kompensator. Detail aus Abb. 46 a. Vergrößerung 100fach.

Der Haverskanal $(*)$ weist Howshipsche Lakunen $(\rightarrow)$ als Zeichen einer Resorption von innen heraus auf. Deutlich erkennbar sind die für Howshipsche Lakunen typischen Doppelkonturierungen $(\rightarrow)$.

* Haverskanal, a $\rightarrow$ : Howshipsche Lakune.

Abb. 46 d: Grab 15; 10 bis 16 Monate. Geschlecht unbestimmbar. Betrachtung eines Knochendünnschliffs $(50 \mu \mathrm{m})$ aus dem rechten Femur im polarisierten Durchlicht unter Benutzung eines Hilfsobjekts Rot 1. Ordnung (Quarz) als Kompensator. Vergrößerung 100fach.

Der Ausschnitt zeigt eine normale Resorptionshöhle im Bereich der Compacta. Die Ränder sind gleichmäßig und glatt konturiert. 

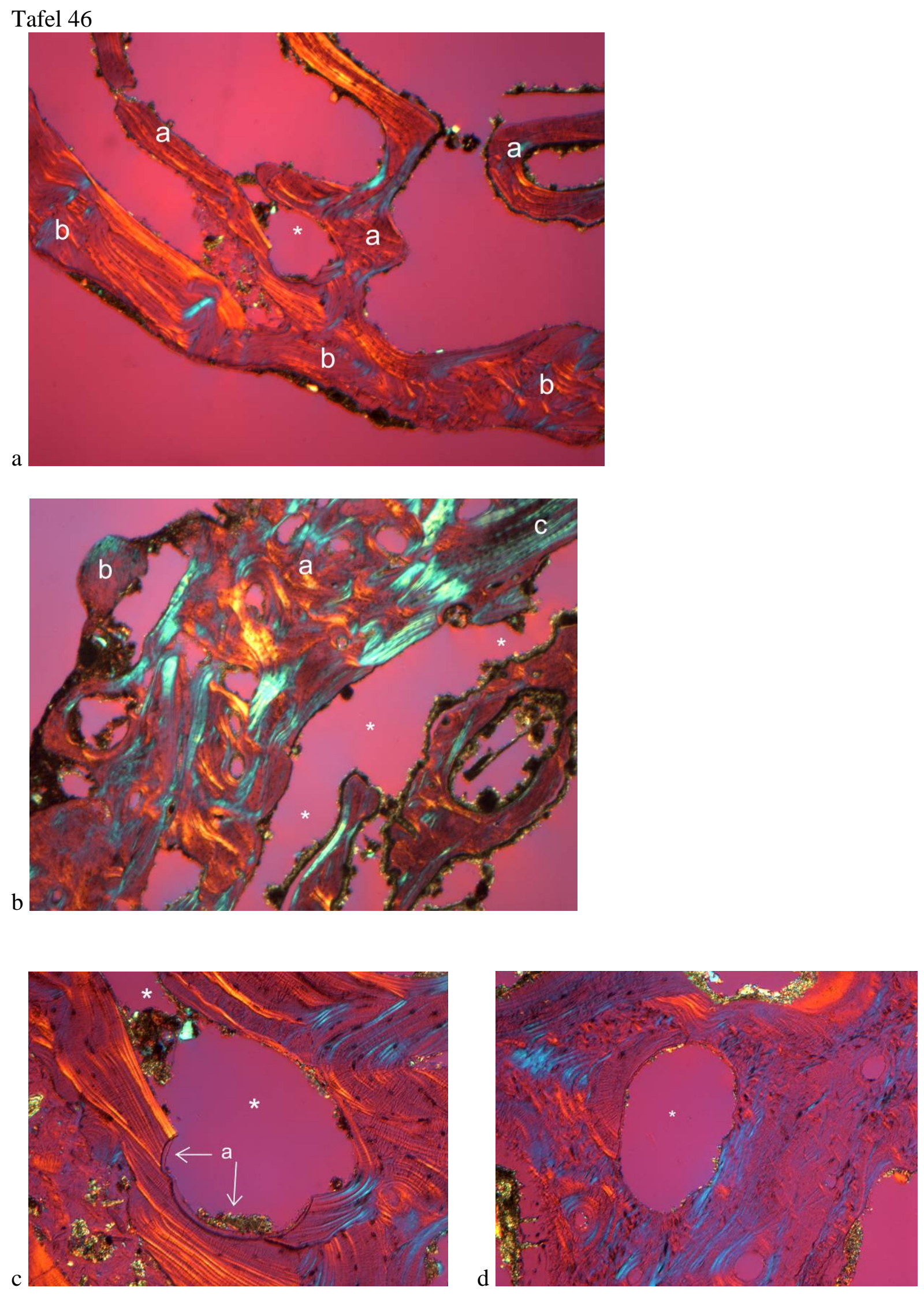
Abb. 47 a: Grab 300 A; 2 bis 3 Jahre. Geschlecht unbestimmbar. Röntgenaufnahme der rechten und linken Fibula bzw. Tibia beidseits. Strahlengang ap, 50 kV, Belichtungszeit 4,5 Min.

Die linke Seite (im Bild rechts) ist normal konfiguriert. Die Knochen der rechten Seite sind nach medial gebogen und weisen eine Verdickung der Corticalis vor allem im mittleren Diaphysenbereich auf.

Abb. 47 b: Grab 15; 10 bis 16 Monate. Geschlecht unbestimmbar. Röntgenaufnahme des rechten und linken Femurs. Strahlengang ap, 45 kV, Belichtungszeit 4,2 Min.

Die Corticalis erscheint beidseits sehr aufgelockert und dünn. Die Knochen sind insgesamt sehr grazil.

Abb. 47 c: Grab 15; 10 bis 16 Monate. Geschlecht unbestimmbar. Röntgenaufnahme der rechten und linken Tibia. Strahlengang ap, $45 \mathrm{kV}$, Belichtungszeit 4,2 Min.

Die Corticalis erscheint beidseits sehr aufgelockert und dünn. Die Knochen sind insgesamt sehr grazil. 
Tafel 47
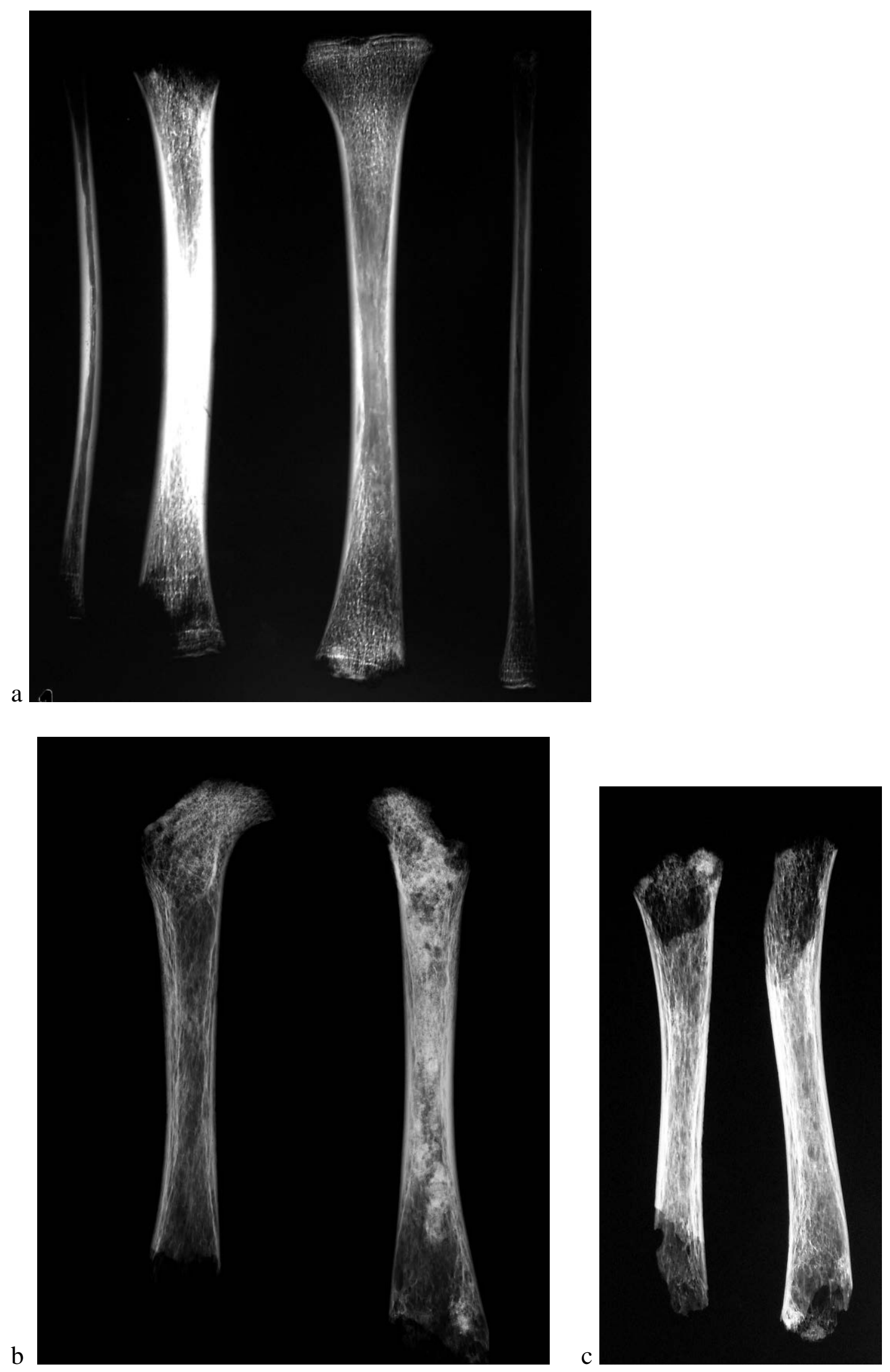
Abb. 48 a und b: Grab 340; 1 bis 2 Jahre. Geschlecht unbestimmbar. Betrachtung eines Knochendünnschliffs $(70 \mu \mathrm{m})$ aus dem distalen Diaphysendrittel (Abb. 48 a) bzw. aus der Diaphysenmitte (Abb. 48 b) des rechten Femurs. Dünnschliffübersicht. Vergrößerung 3fach.

Abb. 48 c: Grab 340; 1 bis 2 Jahre. Geschlecht unbestimmbar. Betrachtung eines Knochendünnschliffs $(70 \mu \mathrm{m})$ aus der Diaphysenmitte der rechten Tibia. Dünnschliffübersicht. Vergrößerung 3fach.

Abb. 48 d: Grab 340; 1 bis 2 Jahre. Geschlecht unbestimmbar. Betrachtung eines Knochendünnschliffs $(50 \mu \mathrm{m})$ aus dem distalen Diaphysendrittel des rechten Femurs im polarisierten Durchlicht unter Benutzung eines Hilfsobjekts Rot 1. Ordnung (Quarz) als Kompensator. Vergrößerung 16fach.

Die Corticalis ist sehr schmal ausgebildet. a: Markröhre, b: äußere Oberfläche.

Abb. 48 e: Grab 340; 1 bis 2 Jahre. Geschlecht unbestimmbar. Betrachtung eines Knochendünnschliffs $(50 \mu \mathrm{m})$ aus der rechten Tibia im polarisierten Durchlicht unter Benutzung eines Hilfsobjekts Rot 1. Ordnung (Quarz) als Kompensator. Vergrößerung 16fach.

Die Corticalis ist sehr schmal ausgebildet. Es ist das Korrelat der im Röntgenbild erscheinenden Doppellamelle erkennbar (s. Katalog).

a: Markröhre, b: äußere Oberfläche.

Abb. 48 f: Grab 340; 1 bis 2 Jahre. Geschlecht unbestimmbar. Betrachtung eines Knochendünnschliffs $(70 \mu \mathrm{m})$ aus dem distalen Diaphysendrittel des rechten Femurs im polarisierten Durchlicht unter Benutzung eines Hilfsobjekts Rot 1. Ordnung (Quarz) als Kompensator. Vergrößerung 100fach.

Detail der sehr schmalen Corticalis. Zwischen innerer und äußerer Generallamelle befindet sich kaum kompakter Knochen. Diese Veränderungen sind als Zeichen einer Inaktivitätsatrophie zu werten.

a: innere Generallamelle, b: äußere Generallamelle.

Abb. 48 g: Grab 340; 1 bis 2 Jahre. Geschlecht unbestimmbar. Betrachtung eines Knochendünnschliffs $(70 \mu \mathrm{m})$ aus dem distalen Diaphysendrittel des rechten Femurs im polarisierten Durchlicht unter Benutzung eines Hilfsobjekts Rot 1. Ordnung (Quarz) als Kompensator. Vergrößerung 100 fach.

Knochendünnschliff des rechten Femurs, distales Drittel. In der schmalen Corticalis sind einzelne Primärosteone (c) auszumachen.

a: Markröhre, b: äußere Oberfläche, c: Primärosteone. 
Tafel 48
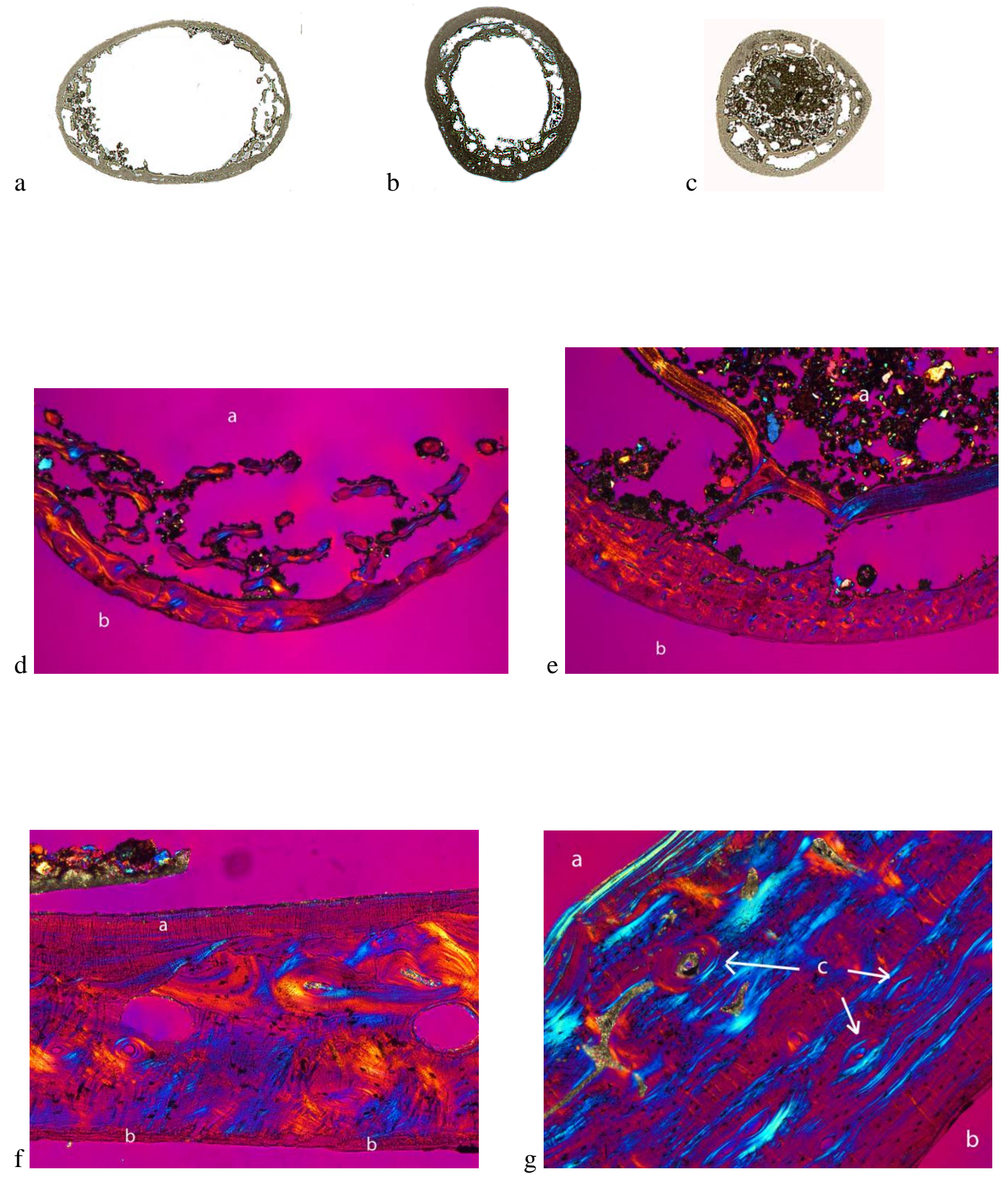
Abb. 49 a: Grab 88 A; 2,5 bis 4,5 Jahre. Geschlecht unbestimmbar. Os parietale rechts. Ansicht der Lamina interna. Atypische Gefäßimpressionen $(\rightarrow)$ und zungenförmige Platten $\left(^{*}\right)$ weisen auf einen endocranial abgelaufenen hämorrhagischen Prozess hin.

Abb. 49 b: Grab 72; 1,5 bis 2,5 Jahre. Geschlecht unbestimmbar. Maxilla links, Buccalansicht. Die Oberfläche ist an der Dorsalseite über die Norm verstärkt porös $(\rightarrow)$. a: Zahn 65, b: Zahn 64, *: Foramen infraorbitale links.

Abb. 49 c: Grab 88 A; 2,5 bis 4,5 Jahre. Geschlecht unbestimmbar. Maxilla rechts, Dorsolateralansicht. Die Oberfläche ist an der Dorsalseite oberhalb der eröffneten Alveole des Zahnes 16 über die Norm verstärkt porös.

a: Processus frontalis maxillae, b: Sutura zygomaticomaxillaris, +: Facies orbitalis, *: Alveole des Zahnes 16, $\rightarrow$ poröse Oberfläche.

Abb. 49 d: Grab 88 A; 2,5 bis 4,5 Jahre. Geschlecht unbestimmbar. Maxilla rechts, Cranialansicht auf Orbitaboden $(\rightarrow)$ und Seitenwand (b). Der Orbitaboden weist ebenso wie die Seiten- und Hinterfläche der Maxilla eine verstärkte Porosität auf. a: Processus zygomaticus, b: Seitenfläche der Maxilla, c: Sutura palatina, d: Ventralseite der Maxilla, $\rightarrow$ poröse Oberfläche des Orbitabodens. 
Tafel 49
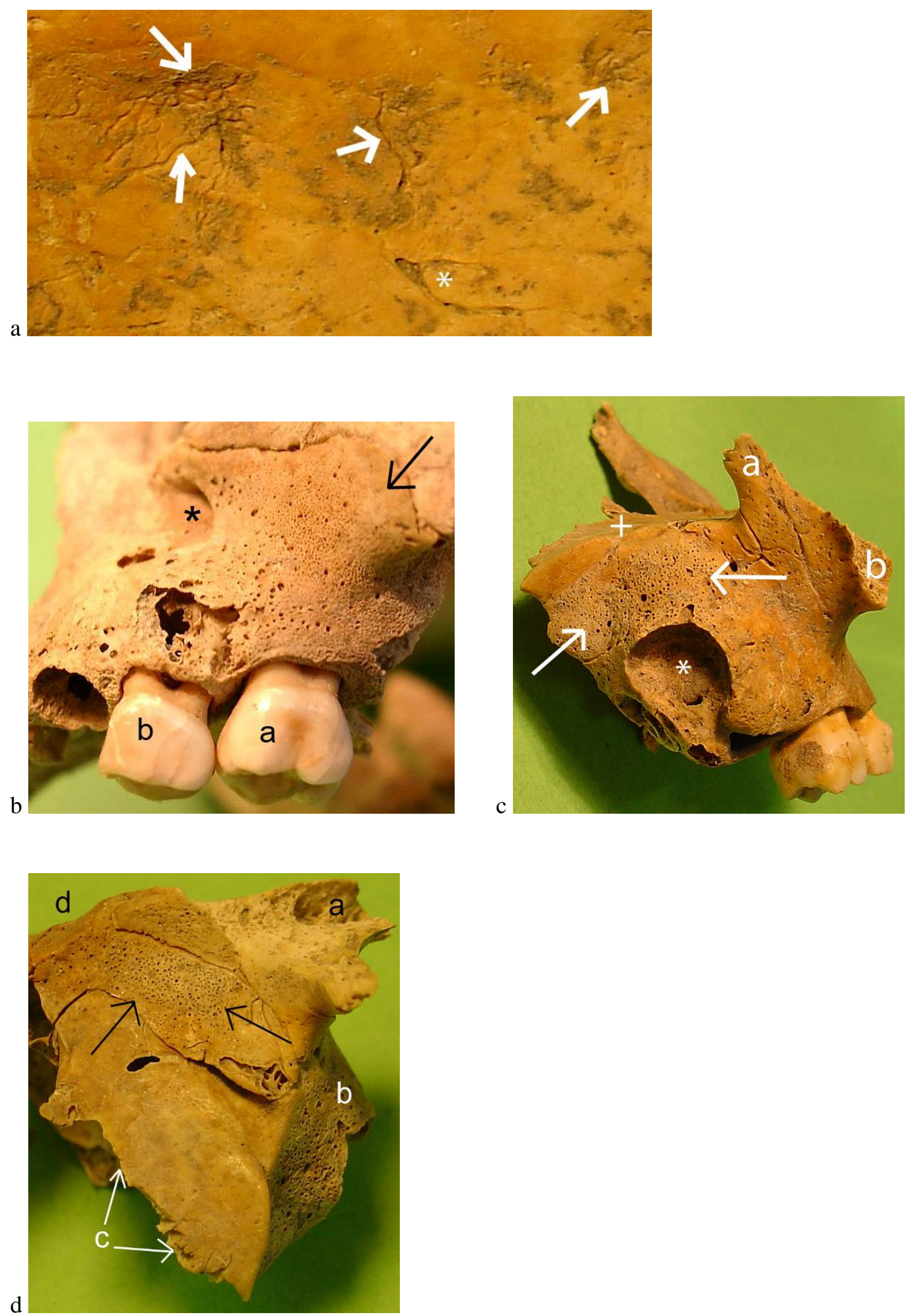
Abb. 50 a: Grab 65 A; 3 bis 4 Jahre. Geschlecht unbestimmbar. Maxilla rechts, Ausschnitt des Alveolarfortsatzes von buccal. Am Rande der Alveole des Zahnes $53(\rightarrow)$ ist die Oberfläche verstärkt porös. Dies ist als Zeichen eines hämorrhagischen Geschehens im Rahmen eines Skorbuts zu werten.

*: Zahn 54, $\rightarrow$ : Alveole des Zahnes 53.

Abb. 50 b: Grab 220; 9,5 bis 12,5 Jahre. Geschlecht weiblich. Mandibula links, Buccalansicht. Die verstärkte Porosität am Rande der Alveolen (a) der Zähne 74 und 75 ist das Zeichen eines hämorrhagischen Geschehens im Rahmen eines Skorbuts.

Abb. 50 c: Grab 72; 1,5 bis 2,5 Jahre. Geschlecht unbestimmbar. Lateralansicht des Schädels von rechts. An der Außenfläche der Ala major ossis sphenoidalis ist eine poröse Auflagerung $(\rightarrow)$ zu erkennen.

a: Os temporale mit Processus zygomaticus, b: Os frontale, c: Öffnung der Orbita.

Abb. 50 d: Grab 301; 4,5 bis 6 Jahre. Geschlecht wahrscheinlich weiblich. Dorsalansicht des distalen Diaphysenabschnitts des linken Femurs. Die Metaphysenplatte ist als Zeichen der Wachstumsstörung im Rahmen eines Skorbuts nach hinten "gekrempelt". Es handelt sich um eine Osteophytenbildung am Rand der Wachstumszone.

Abb. 50 e: Grab 6 B; 0,5 bis 1 Jahr. Geschlecht unbestimmbar. Caudalansicht der rechten Ala minor des Os sphenoidale. Im Bereich der Sutura sphenofrontalis $(\rightarrow)$ befindet sich eine poröse Auflagerung. 
Tafel 50
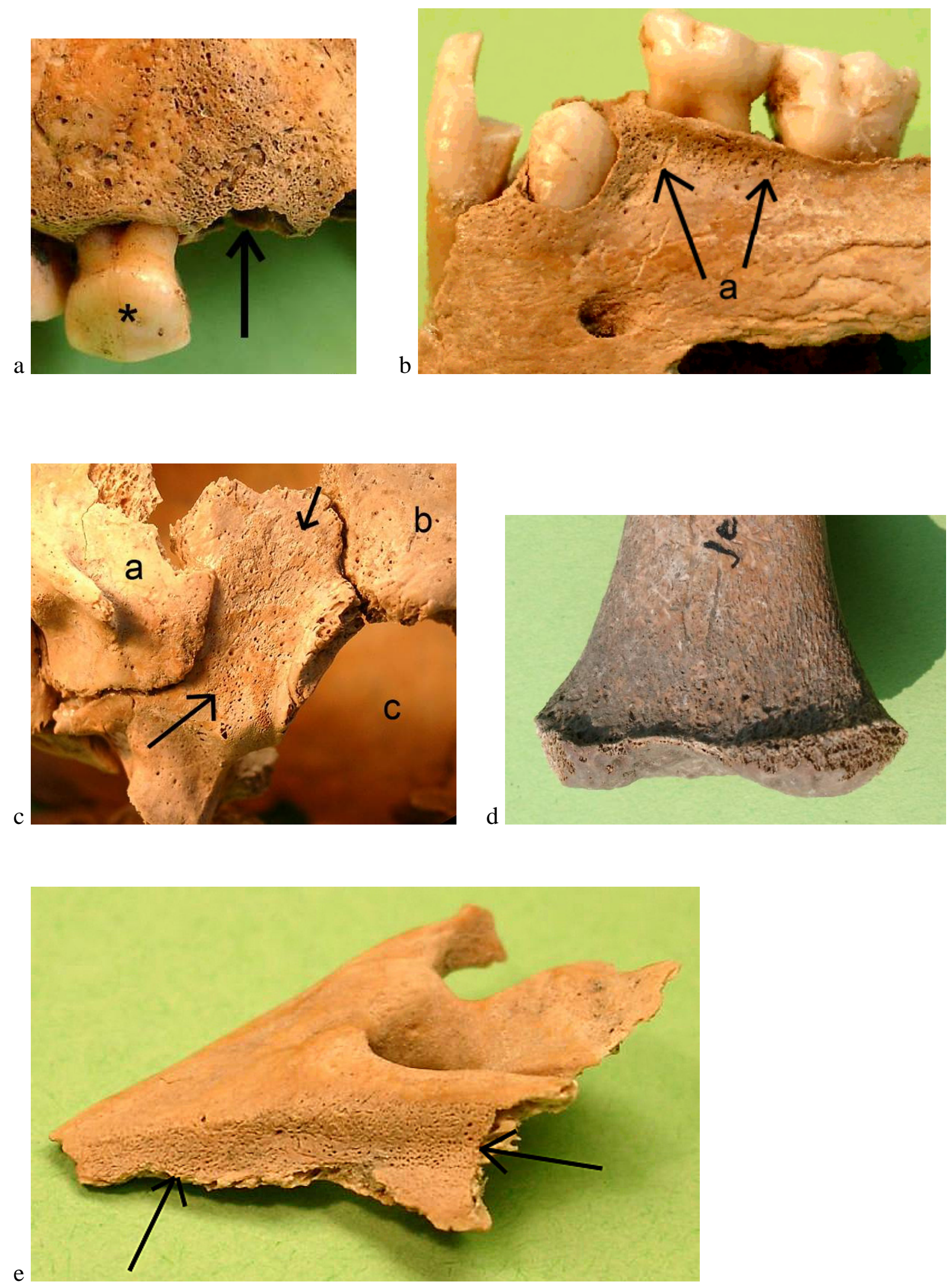


\section{$\underline{\text { Literaturverzeichnis }}$}

Albrecht B, Eschenhagen W, Reindel T, Unger-Kunz M: Staaten, Länder und Gebiete; in: Der Fischer Weltalmanach 2003; hrsg. v. von Baratta M; Fischer Taschenbuch Verlag, Frankfurt/M. 2002

ARNOLD K: Die Einstellung zum Kind im Mittelalter; in: Mensch und Umwelt im Mittelalter; hrsg. v. Herrmann B; Fischer Verlag, Frankfurt/M. 1989, 53-64

ASCHOFF L: Spezielle pathologische Anatomie. Ein Lehrbuch für Studierende und Ärzte 2, 4. Auflage; Verlag von Gustav Fischer, Jena 1919

BARLOW T (1883): On cases described as "acute rickets" which are probably a combination of scurvy and rickets, the scurvy being an essential and the rickets a variable element. Medico-Chirurgial Transactions $\underline{66}$, 159-219

BARLOW T (1895 a): Der infantile Scorbut und seine Beziehung zur Rachitis; Zentralbl. Inn. Med. 21, 505-529

BARLOW T (1895 b): Der infantile Scorbut und seine Beziehung zur Rachitis; Zentralbl. Inn. Med. 22, 529-536

BARNES E: Developmental defects of the axial skeleton in paleopathology, University press of Colorado, Niwot 1994

BÁTORA J (1986): Výskum pohrebiska zo staršej doby bronzovej v Jelšovciach. Arch Rozhledy $\underline{38}$, 263-274 
BÁTORA J: Das Gräberfeld von Jelšovce/ Slowakei. Ein Beitrag zur Frühbronzezeit im nordwestlichen Karpatenbecken; Prähistorische Archäologie in Südosteuropa 16; ; hrsg. v. Hänsel B; Verlag Oetker/ Voges, Kiel 2000

BÁTORA J, SCHULTZ M: Zum Gesundheitszustand der frühbronzezeitlichen Populationen des Gräberfeldes von Jelšovce / Slowakei; in: Mensch und Umwelt während des Neolithikums und der Frühbronzezeit in Mitteleuropa. Ergebnisse interdisziplinärer Zusammenarbeit zwischen Archäologie, Klimatologie, Biologie und Medizin; hrsg. v. Lippert A, Schultz M, Shennan S, Teschler-Nicola M; Verlag Marie Leidorf GmbH, Rahden/Westf. 2001, 307-315

BEHRINGER W: Kulturgeschichte des Klimas. Von der Eiszeit bis zur globalen Erwärmung; Verlag C.H. Beck, München 2007

BENNION E: Alte Medizinische Instrumente. Ernst Klett Verlag, Stuttgart 1980

BERNER M: Demographie des frühbronzezeitlichen Gräberfeldes Franzhausen I, Niederösterreich; in: Demographie der Bronzezeit, Paläodemographie - Möglichkeiten und Grenzen; hrsg. v. Dobiat C, Leidorf K; Verlag Marie Leidorf GmbH, Espelkamp, Rahden/ Westf. 1997, 35-42

BILGI Ö (1984): Metal objects from İkiztepe - Turkey. Beitr. zur Allg. und Vergl. Archäologie $\underline{6}$, 31-97

BOCQUET-APPEL JP, MASSET C (1982): Farwell to Paleodemography. J Hum Evol 11, 321333 
BoenNinghaus HG, LENARZ T : Hals-Nasen-Ohren-Heilkunde für Studierende der Medizin; 11.Auflage; Springer-Verlag, Berlin 2001

BOUZEK J: Climatic changes and central European prehistory; in: Climatic changes in later prehistory; hrsg. v. Harding AF; Edinburgh University press, Edinburgh 1982, 179-191

BouZEK J (1993): Climatic changes: New archaeological evidence from the Bohemian Karst and other Areas. Antiquity 67, 386-393

BOUZEK J: Klimatische Entwicklung im Äneolithikum und in der Frühbronzezeit in Böhmen und Mähren; in: Mensch und Umwelt während des Neolithikums und der Frühbronzezeit in Mitteleuropa. Ergebnisse interdisziplinärer Zusammenarbeit zwischen Archäologie, Klimatologie, Biologie und Medizin; hrsg. v. Lippert A, Schultz M, Shennan S, TeschlerNicola M; Verlag Marie Leidorf GmbH, Rahden/ Westf. 2001, 21-26

BRAULKE F: Ergebnisse der paläopathologischen Untersuchungen an den Postcranien der frühneolithischen Erwachsenenskeletten aus Wandersleben, Kreis Gotha. Med. Diss. Göttingen 2004

BRICKLEY M, IVES R (2006): Skeletal Manifestations of Infantile Scurvy. Am J Phys Anthropol 129, 163-172

BROCHER JEW, WILLERT HG: Fehlbildungen der Wirbelsäule; in: Differenzialdiagnose der Wirbelsäulenerkrankungen, 6.Auflage, Georg-Thieme-Verlag, Stuttgart 1980, 57-92 
BROTHWELL DR: Digging up bones, 3. Auflage; British Museum of Natural History; Oxford University Press, Oxford 1981

BYERS S (1991): Calculation of age of formation of radiopaque transverse lines. Am J Phys Anthropol 85, 339-343

CARLI-ThIEle P: Spuren von Mangelerkrankungen an steinzeitlichen Kinderskeleten, Fortschritte in der Paläopathologie und Osteologie $\underline{1}$; hrsg. v. Schultz M; Verlag Erich Goltze, Göttingen 1996

CARLi-Thiele P, Schultz M: Wechselwirkungen zwischen Mangel- und Infektionskrankheiten des Kindesalters bei neolithischen Populationen; in: Mensch und Umwelt während des Neolithikums und der Frühbronzezeit in Mitteleuropa. Ergebnisse interdisziplinärer Zusammenarbeit zwischen Archäologie, Klimatologie, Biologie und Medizin; hrsg. v. Lippert A, Schultz M, Shennan S, Teschler-Nicola M; Verlag Marie Leidorf GmbH, Rahden/ Westf. 2001, 273-285

DETKEN S: Krankhafte Veränderungen an den menschlichen Skeleten aus dem spätantikenfrühmittelalterlichen Reihengräberfeld von Barbing, Niederbayern. Med. Diss. Göttingen 1989

FEREMBACH D, SCHIDETZKY I, STLOUKAL M (1979): Empfehlungen für die Alters- und Geschlechtsdiagnose an Skelett. Homo $\underline{30}$ (2), 1-32

FISCHER K: Pathologie der Erythrozyten; in: Kinderheilkunde; hrsg. v. von Harnack GA; Springer-Verlag, Berlin 1974, 232-238 
FrAenKel E: Die Möller-Barlowsche Krankheit, Archiv und Atlas der normalen und pathologischen Anatomie in typischen Röntgenbildern, Fortschritte auf dem Gebiete der Röntgenstrahlen, Ergänzungsband 18; hrsg. v. Albers-Schönberg, Verlag Lucas Gräfe \& Stillem, Hamburg 1908

FrAenKel E, LOREY A: Die Rachitis im Röntgenbild, Archiv und Atlas der normalen und pathologischen Anatomie in typischen Röntgenbildern, Fortschritte auf dem Gebiete der Röntgenstrahlen, Ergänzungsband 22; hrsg. v. Albers-Schönberg, Verlag Lucas Gräfe \& Stillem, Hamburg 1910

FURMÁNEK V, VELIAČIK L, VLADÁR J: Die Bronzezeit im Slowakischen Raum. Prähistorische Anthropologie in Südosteuropa 15; hrsg. v. Hänsel B; Verlag Marie Leidorf GmbH, Rhaden/ Westf. 1999

GÖRSDORF J: Interpretation der Datierungsergebnisse von Menschenknochen aus dem Gräberfeld Jelšovce; in: Das Gräberfeld von Jelšovce/ Slowakei. Ein Beitrag zur Frühbronzezeit im nordwestlichen Karpatenbecken; Prähistorische Archäologie in Südosteuropa 16; hrsg. v. Hänsel B; Verlag Oetker/ Voges, Kiel 2000, 565-570

GÖTZ W: Histologische Untersuchungen an Cribra orbitalia- ein Beitrag zur Paläopathologie der Orbitadaches. Med. Diss. Göttingen 1988

Gresky J, Roumelis N, Schulz C, Schultz M: Diachrone Untersuchungen zur Epidemiologie der Erkrankungen des Mittelohres in mitteleuropäischen Populationen unter geschlechtsspezifischen Aspekten. Vortrag gehalten beim 5. Kongress der GfA in Potsdam am 19.9.2003 
HARDIG AF: European Societies in the Bronze Age. Cambridge World Archeology; Cambridge University Press, Cambridge 2000

HART C, LESSING O: Der Skorbut der kleinen Kinder; Enke Verlag, Stuttgart 1913

HeISTER L: Chirurgie - In welcher Alles was zur Wund -Arzney gehöret, abgehandelt und vorgestellt wird. Wund - Arzney Chirurgie, Nürnberg 1719; Reprint- Verlag Leipzig, Leipzig 1983

HEMPEN CH: Taschenatlas Akupunktur; Georg Thieme Verlag, Stuttgart 1998

HIPPOKRATES: Über Luft- Wasser- und Ortsverhältnisse. In: Antike Heilkunst, ausgewählte Texte aus medizinischen Texten der Griechen und Römer; hrsg. v. Kollesch J, Nickel D; Philipp Reclam jun., Stuttgart 1994, 121-137

Huvos AG: Bone Tumors. Diagnosis, Treatment and Prognosis; W.B. Saunders Company, Philadelphia 1979

JAFFE HL: Metabolic, Degenerative and Inflammatory Diseases of Bones and Joints. Urban \& Schwarzenberg, München 1972

JANI L, KRÄMER KL: Kinderorthopädie; in: Pädiatrie; hrsg. v. Speer CP, Gahr M; SpringerVerlag, Berlin 2001, 907-910 
JoHnstON FE (1962): Growth of the Long Bones of Infants and Young Children at Indian Knoll; Am J Phys Anthropol 20, 249-254

JunDT G: Knochen; in: Pathologie; hrsg. v. Böcker W, Denk H, Heitz U; Urban und Fischer Verlag, München 2001, 971-981

KLINGEBIEL T: Solide Tumoren; in: Pädiatrie; hrsg. v. Speer CP, Gahr M; Springer-Verlag, Berlin 2001, $726-729$

KLOIBER Ä: Die Gräberfelder von Lauriacum. Das Ziegelfeld. Oberösterreichisches Landesmuseum, Linz 1957

KLOSE E: Kinderheilkunde. Ärztliche Bücherei für Fortbildung und Praxis, Band III; hrsg. v. Verband der Ärzte Deutschlands; Verlag der Buchhandlung des Verlags der Ärzte Deutschlands, Leipzig 1919

KosEnOV W: Erkrankungen der Ohren; in: Kinderheilkunde; hrsg. v. von Harnack GA; Springer-Verlag, Berlin 1973, 285-286

KREUTZ K: Ätiologie und Epidemiologie von Erkrankungen des Kindesalters bei der bajuwarischen Population von Straubing (Niederbayern). Beiträge zur Paläopathologie. hrsg. v. Schultz M; Cuvillier Verlag, Göttingen 1997

Kriener S, FELLBAum C, HANSMANN ML: Blut, Knochenmark und lymphatisches System; in: Pathologie; hrsg. v. Böcker W, Denk H, Heitz U; Urban und Fischer Verlag, München 2001, 486-492 
KUDLÁČEK J (1958): Záchranný výskum v Jelovciach (okr Nitra) r. 1952, Slov Arch $\underline{6}$, 50-65

KÜBLER W: Vitaminmangelerkrankungen; in: Kinderheilkunde; hrsg. v. von Harnack GA; Springer-Verlag, Berlin 1974, 85-89

KÜNZEL W, TOMAN J: Kinderzahnheilkunde; Dr. Alfred Hüthig Verlag, Heidelberg 1985

Lyons AS, Petrucelli RJ: Die Geschichte der Medizin im Spiegel der Kunst; DuMont Buchverlag, Köln 1980

Mc HENRY HM, SCHULZ PD (1976): The association between Harris lines and enamel hypoplasia in prehistoric Carlifornia Indians. Am J Phys Anthropol 44, 507-512

Mensforth RP, LOVEJoy CO, LALlo JW, ARmelagos GJ (1978): The role of constitutional factor, diet, and infectious disease in the etiology of porotic hyperostosis and periostal reactions in prehistoric infants and children. Med Anthropol 2, 1-59

MirRA JB: Bone Tumors. Diagnosis and Treatment; JB Lippincott Company, Philadelphia/ USA 1980

MÖLLER JO (1862): Zwei Fälle von Acuter Rachitis; Königsberger Med Jb 3, 222-239

MoODIE RL: Paleopathology. An Introduction tot he Study of Ancient Evidences of Disease. University of Illinois Press, Urbana/ Illinois 1923 
MurRAY JONES P: Heilkunst des Mittelalters in illustrierten Handschriften; Belser Verlag, Stuttgart 1999

NEUGEBAuER JW: Bronzezeit in Ostösterreich. (Wissenschaftliche Schriftreihe Niederösterreich 98-101); Verlag Niederösterreichisches Pressehaus, St. Pölten 1994

NEUGEBAUER JW: Siedlungen und zugehörige Friedhöfe der Früh- und Mittelbronzezeit in Franzhausen, Herzogenburg, Pottenbrunn und Gemeinlebarn; in: Demographie der Bronzezeit. Paläodemographie - Möglichkeiten und Grenzen; hrsg. v. Dobiat C, Leidorf K; Verlag Marie Leidorf GmbH, Espelkamp, Rahden/ Westfalen 1997, 35-42

Neugebauer JW, Neugebauer- Maresch C: Das frühbronzezeitliche Hockergräberfeld in urgeschichtlicher Sicht; in: Mensch und Kultur der Bronzezeit; hrsg. v. Windl J, Neugebauer JW, Teschler-Nikola M, Neugebauer-Maresch C; Kat. Niederöster. Landesmuseum. N.F. 208, 1988, 16-36

ORTNER DJ: Identification of Pathological Conditions in human skeletal remains; Academic press San Diego, San Diego 2003

ORTNER DJ, ERICKSEN MF (1997): Bone changes in the human skull probably resulting from scurvy in infancy and childhood. Int J Osteoarch 7 (3), 212-220

ORTNER DJ, MAYS S (1998): Dry-Bone manifestations of rickets in infancy and early childhood. Int J Osteoarch $\underline{8(1)}, 45-55$ 
ORTNER DJ, ButLER W, CAFARELla J, MiLligan L (2001): Evidence of probable scurvy in subadults in North America. Am J Phys Anthropol 114, 343-351

PERIZONIUS WRK, POT T (1981): Diachronic Dental Research on Human Skeletal Remains Excavated in the Netherlands; in: Dorestad's Cemetery on 'the Heul'. Berichten van de Rijksdienst voor het Oudheidkundig Bodemonderzoek $\underline{31}$, 369-413

PODBORSKÝ V: Heimatkunde des Mährischen Landes und der Leute. Die Vorgeschichte Mährens (dt. Zusammenfassung). NR 3, Brünn 1993, 523-525

POHL M: Krankhafte Veränderungen an den menschlichen Skeleten aus dem bajuwarischen Reihengräberfeld von Harting, Niederbayern. Med. Diss. Göttingen 1989

RITTER J: Hämatologie; in: Pädiatrie; hrsg. v. Speer CP, Gahr M; Springer-Verlag, Berlin 2001, 661-689

RÜsTER D: Der Chirurg (historische Berufsbilder). Ein Beruf zwischen Ruhen und Vergessen. Edition Leipzig, Leipzig 1993

SCHACHERMEYR F: Die minoische Kultur des alten Kreta. W Kohlhammer Verlag, Stuttgart 1964

SCHEFFLER F, SCHACHTSCHABEL P: Lehrbuch der Bodenkunde. 15. Auflage; Spektrum, Berlin 2002 
SCHLOMM T: Spuren pathologischer Prozesse an den menschlichen Schädeln aus dem frühmittelalterlichen Reihengräberfeld von Barbing-Kreuzhof unter besonderer Berücksichtigung der unspezifischen und tuberkulösen Entzündungen im Bereich der Meningen und der pneumatischen Schädelräume, Teil 1; Med. Diss. Göttingen 2000

SCHULTZ M: Krankhafte Veränderungen an den menschlichen Skeleten aus dem merowingischen Reihengräberfeld von Kleinlangheim/ Ldkr. Kitzingen - Eine bevölkerungsbiologische Untersuchung. Nat. Diss. Frankfurt/M. 1978

SCHULTZ M: Umwelt und Krankheit des vor- und frühgeschichtlichen Menschen; in: Kindlers Enzyklopädie der Mensch 2̧; hrsg. v. Wendt H, Loacker N; Kindler Verlag, Zürich 1982, 259312

SCHULTZ M: Spuren unspezifischer Entzündungen an prähistorischen und historischen Schädeln. Med. Habil.-Schr. Göttingen 1987

SchUlTZ M: Paläopathologische Diagnostik; in: Anthropologie. Handbuch der vergleichenden Biologie des Menschen 1: Wesen und Methoden der Anthropologie; hrsg. v. Knußmann R; Fischer Verlag, Stuttgart 1988 a, 480-496

SCHULTZ M: Methoden der Licht und Elektronenmikroskopie; in: Knußmann Anthropologie. Handbuch der vergleichenden Biologie des Menschen 1: Wesen und Methoden der Anthropologie; hrsg. v. Knußmann R; Fischer Verlag, Stuttgart 1988 b, 698-730

SCHULTZ M (1988/89): Erkrankungen des Kindesalters bei der frühbronzezeitlichen Population von Hainburg/ Niederösterreich. Mitt Anthrop Ges Wien 118/119; 369-380 
ScHulTZ M (1989 a): Erkrankungen des Kindesalters in der Bronzezeit. Niedersächsisches Ärzteblatt 9/ 1989, 28-34

SCHULTZ M (1989 b): Ergebnisse osteologischer Untersuchungen an den mittelalterlichen Kinderskeletten unter besondere Berücksichtigung anatolischer Populationen. Anthropol Anz $\underline{47(1)}, 39-50$

SCHULTZ M (1989 c): Zur Morbidität neolithischer Populationen. Ein Beitrag zur Paläopathologie, Homo $\underline{40}$, 81-98

SCHULTZ M: Erkrankungen des Kindesalters bei der frühbronzezeitlichen Population von İkiztepe (Türkei); in: Gedenkschrift für Jürgen Driehaus; hrsg. v. Andraschko FM, Teegen WR; Verlag Philipp v. Zabern, Mainz 1990, 83-90

SCHULTZ M (1993): Spuren unspezifischer Entzündungen an prähistorischen und historischen Schädeln. Ein Beitrag zur Paläopathologie. Anthropologisches Forschungsinstitut Aesch und Anthropologische Gesellschaft Basel. Anthropologische Beiträge, Bd. 4A, 1-84; Bd. 4B, Taf. 1-51. Aesch

SCHULTZ M: Ergebnisse mikroskopischer Untersuchungen an archäologischen Skelettfunden. Ein Beitrag zur Paläopathologie; in: Beiträge zur Archäozoologie und Prähistorischen Anthropologie, 8. Arbeitstreffen der Osteologen, Konstanz 1993, im Andenken an Joachim Boessneck, Forschungen und Berichte zur Vor- und Frühgeschichte in Baden-Württemberg 53; hrsg. v. M. Kokabi und J. Wahl, Konrad Thies Verlag, Stuttgart 1994 a, 107-116 
SCHULTZ M: Leben, Krankheit und Tod - Skelettfunde als Spiegel der Lebensbedingungen; in: Bronzezeit in Deutschland; hrsg. v. Jockenhövel A, Kubach W; Nikol Verlagsgesellschaft mbH \& Co. KG, Hamburg 1994 b, 15-17

SCHULTZ M: Krankheit und Tod im Kindesalter bei frühbronzezeitlichen Populationen; in: Mensch und Umwelt während des Neolithikums und der Frühbronzezeit in Mitteleuropa. Ergebnisse interdisziplinärer Zusammenarbeit zwischen Archäologie, Klimatologie, Biologie und Medizin. Internationale Archäologie; hrsg. v. Lippert A, Schultz M, Shennan S, TeschlerNicola M; Verlag Marie Leidorf GmbH, Rahden/ Westfalen 2001 a, 287-305

SCHULTZ M (2001 b): Paleohistopathology of Bone: A New Approach to the Study of Ancient Disease. Yearb Phys Anthropol 44, 106-147

SCHULTZ M: Light Microscopic Analysis in Skeletal Paleopathology; in: Identification of Pathological Conditions in human skeletal remains; hrsg. v. Ortner DJ; Academic press San Diego, San Diego 2003, 73-109

Schultz M, DROMMER R: Möglichkeiten der Präparatherstellung aus dem Gesichtsschädelbereich für die mikroskopische Untersuchung unter Verwendung neuer Kunststofftechniken; in: Experimentelle Mund-Kiefer-Gesichts-Chirurgie; hrsg. v. Hoppe W; Thieme Verlag, Stuttgart 1983, 95-97

Schultz M, SCHMIDT-Schultz T (1994): Krankheiten des Kindesalters in der mittelalterlichen Population von Pergamon. Ergebnisse einer paläopathologischen Untersuchung, Ist Mitt $\underline{44}, 181-201$ 
Schultz M, SchMidT-Schultz T: Ätiologie und Epidemiologie der Krankheiten des Kindesalters in der Bronzezeit; in: Beiträge zur Archäozoologie und prähistorischen Anthropologie 2; hrsg. v. May E; Weis \& Partner Verlag, Stuttgart 1999, 257-263

SCHULTZ M, SCHMIDT-SCHULTZ T, KREUTZ K: Ergebnisse der paläopathologischen Untersuchung an den frühbronzezeitlichen Kinderskeleten von Jelšovce (Slowakische Republik); in: Mensch und Umwelt in der Bronzezeit in Europa; hrsg. v. Hänsel B; OetkerVoges-Verlag, Kiel 1998, 77-90

SCHUSTER W Kinderradiologie 1, Springer Verlag, Berlin 1990

SCHWIDETZKY I (1965): Sonderbestattungen und ihre paläodemographische Bedeutung. Homo $\underline{16}$, 230-247

SCUltetus J: Wund - Arzneyisches Zeug - Hauß; Faksimile Druck der Scultetus - Ausgabe von 1666. (Forschungen zur Geschichte der Stadt Ulm 14); hrsg. v. Scultetus - Gesellschaft e.V. Ulm/ Donau und Stadtarchiv Ulm; Kommissionsverlag W. Kohlhammer, Stuttgart 1988

Spranger JW, LANGER LO, Wiedemann HR: Bone Dysplasias. An Atlas of Constitutional Disorders of Skeletal Development. WB Saunders Company, Philadelphia 1974

STAEMMLER M: Lehrbuch der speziellen pathologischen Anatomie; Walter de Gruyter Verlag, Berlin 1958, 32-34

STEINBOCK RT: Paleopathological Diagnosis and Interpretation. Bone Diseases in ancient human populations. Thomas Books, Springfield/ Illinois 1976 
STLOUKAL M, HANÁKOVÁ H (1978): Die Länge der Längsknochen altslawischer Bevölkerungen unter besonderer Berücksichtigung der Wachstumsfrage. Homo 29, 53-69

SuDHOFF K: Beiträge zur Geschichte der Chirurgie im Mittelalter, Erster Teil; Verlag von Johann Ambrosius Barth, Leipzig 1914

SZILVÁsSY J: Altersdiagnose am Skelett; in: Anthropologie. Handbuch der vergleichenden Biologie des Menschen Band 1; hrsg. v. Knußmann R; Fischer Verlag, Stuttgart 1988, 142443

TEMPLin O: Die Kinderskelete von Bettingen im Kanton Basel-Stadt (Schweiz). Eine paläopathologische Untersuchung. Med. Diss. Göttingen 1993

TESCHLER-NIKOLA M: Bevölkerungsbiologische Aspekte der frühen und mittleren Bronzezeit. in: Neugebauer JW, Die Bronzezeit im Osten Österreichs. Forschungsbericht zur Ur- und Frühgeschichte 13. Österr. AG für Ur- und Frühgeschichte, Verlag Niederösterreichisches Pressehaus, St. Pölten 1994, 167-183

UBELAKER DH: Human skeletal remains. Excavation, Analysis, Interpretation; 2. Auflage; Taraxacum, Washington 1980

WAPler U, CRUBÉZY E, SCHUltz M (2004): Is Cribra Orbitalia Synonymous With Anemia? Analysis and Interpretation of Cranial Pathology in Sudan. Am J Phys Anthropol 123, 333339 
WELLS C: A new approach to paleopathology: Harris’s Lines; in: Diseases in antiquity; hrsg. v. Brothwell D, Sandison AT; Charles C. Thomas, Springfield/ Illinois 1967, 390-404

WIMBERGER HC (1925): Klinisch-radiologische Diagnostik von Rachitis, Skorbut und Lues congenita im Kindesalter. Ergebn. inn. Med. und Kinderheilkd. 28, 264-276

WITTMAACK K: Über die normale und pathologische Pneumatisation des Schläfenbeins einschließlich ihrer Beziehung zu den Mittelohrerkrankungen. Fischer Verlag, Jena 1918

WITTWER-BACKOFEN U: Zur Anwendung von Bevölkerungsmodellen in der Paläodemographie am Beispiel des frühbronzezeitlichen Gräberfeldes von İkiztepe (Türkei); in: Demographie der Bronzezeit. Paläodemographie - Möglichkeiten und Grenzen. Internationale Archäologie 6ㅜㅇ hrsg. v. Rittershofer KF; Verlag Marie Leidorf GmbH, Espelkamp 1997, 65-73

Wood JW, Milner GR, HARPending HC, Weiss KM (1992): The Osteological Paradox. Problems of Inferring Prehistoric Health from Skeletal Samples. Curr Anthropol 33, 343-370 


\section{Danksagung}

Herrn Prof. Dr. Dr. M. Schultz (Arbeitsgruppe Paläopathologie am Zentrum Anatomie der Universität Göttingen) danke ich für die Überlassung des Themas und der von ihm erhobenen Daten, sowie für die Betreuung.

Herrn Prof. Dr. J. Bátora (Archäologie) und Herrn Dr. J. Jakab (Anthropologie) der Slowakischen Akademie der Wissenschaften in Nitra gilt mein Dank für die Überlassung der Skeletfunde.

Frau I. Hettwer-Steeger und Herrn M. Brandt, beide Zentrum Anatomie der Universität Göttingen, danke ich für die Unterstützung bei der Anfertigung der histologischen und rasterelektronenmikroskopischen Präparate sowie des Abgusses.

Der Arbeitsgruppe Paläopathologie am Zentrum Anatomie der Universität Göttingen danke ich für die kollegiale Zusammenarbeit.

Frau Dr. M. Seiler, Herrn Dr. J. Seiler, Herrn M. Seiler und Herrn Dr. P. Weihmann gilt mein Dank für die Unterstützung in technischen Fragen. 